\title{
IDENTIFICATION OF WIND TURBINE TESTING PRACTICES AND INVESTIGATION OF THE PERFORMANCE BENEFITS OF CLOSELY-SPACED LATERAL WIND FARM CONFIGURATIONS
}

by

\section{Sean McTavish}

A thesis submitted to the Faculty of Graduate and Postdoctoral Affairs in partial fulfillment of the requirements for the degree of

\section{Doctor of Philosophy}

in Aerospace Engineering

Ottawa-Carleton Institute for Mechanical \& Aerospace Engineering

\author{
Department of Mechanical \& Aerospace Engineering \\ Carleton University \\ Ottawa, Ontario, Canada
}

(C) Sean McTavish

April 2013 
Library and Archives

Canada

Published Heritage

Branch

395 Wellington Street

Ottawa ON K1A ON4

Canada
Bibliothèque et

Archives Canada

Direction du

Patrimoine de l'édition

395 , rue Wellington

Ottawa ON K1A ON4

Canada
Your file Votre référence

ISBN: 978-0-494-94547-6

Our file Notre référence

ISBN: $978-0-494-94547-6$
NOTICE:

The author has granted a nonexclusive license allowing Library and Archives Canada to reproduce, publish, archive, preserve, conserve, communicate to the public by telecommunication or on the Internet, loan, distrbute and sell theses worldwide, for commercial or noncommercial purposes, in microform, paper, electronic and/or any other formats.

The author retains copyright ownership and moral rights in this thesis. Neither the thesis nor substantial extracts from it may be printed or otherwise reproduced without the author's permission.
AVIS:

L'auteur a accordé une licence non exclusive permettant à la Bibliothèque et Archives Canada de reproduire, publier, archiver, sauvegarder, conserver, transmettre au public par télécommunication ou par l'Internet, prêter, distribuer et vendre des thèses partout dans le monde, à des fins commerciales ou autres, sur support microforme, papier, électronique et/ou autres formats.

L'auteur conserve la propriété du droit d'auteur et des droits moraux qui protege cette thèse. $\mathrm{Ni}$ la thèse ni des extraits substantiels de celle-ci ne doivent être imprimés ou autrement reproduits sans son autorisation.
In compliance with the Canadian Privacy Act some supporting forms may have been removed from this thesis.

While these forms may be included in the document page count, their removal does not represent any loss of content from the thesis.
Conformément à la loi canadienne sur la protection de la vie privée, quelques formulaires secondaires ont été enlevés de cette thèse.

Bien que ces formulaires aient inclus dans la pagination, il n'y aura aucun contenu manquant. 


\section{Abstract}

The current thesis investigates the development of guidelines for testing small-scale wind turbines and identifies a method that can be used to increase the performance of wind farms. The research was conducted using two scaled wind turbine designs. The first design was a three-bladed wind turbine designed in the Department of Mechanical and Aerospace Engineering (MAAE) to operate in a low Reynolds number regime and to generate a thrust coefficient representative of commercial-scale wind turbines. An Eppler E387 airfoil was selected for the wind turbine due to its behaviour at low Reynolds numbers and the chord of the turbine was increased relative to full-scale designs in order to increase the range of Reynolds numbers that could be attained. The second design was a geometrically-scaled version of an existing two-bladed wind turbine with a NACA 0012 airfoil that was originally designed at the Delft University of Technology.

Experiments were conducted in a $0.61 \mathrm{~m} \times 0.81 \mathrm{~m}$ water channel in order to independently evaluate the effects of increasing blockage and Reynolds number on the development of the wind turbine wake. Quantitative dye visualisation was used to identify the position of tip vortex cores relative to the blade tip in order to assess how blockage and Reynolds number effects modified the initial expansion in the near wake. Blockage effects on the wake development were assessed using five wind turbines with diameters ranging from 20 $\mathrm{cm}$ to $40 \mathrm{~cm}$, corresponding to blockage of $6.3 \%$ to $25.4 \%$. The rotors were all operated at a similar tip speed ratio of 6 and a Reynolds number of 23,000 based on the blade tip speed and tip chord. One outcome of the research was the identification of a limit beyond which blockage narrowed the expansion in the near wake of a wind turbine. It was observed that blockage should be maintained at less than $10 \%$ in order to prevent the wake from narrowing artificially due to the flow acceleration around the turbine caused by excessive 
blockage. The experimental results were compared to a freestream computational simulation of the same turbine using the vortex particle method code GENCVP. The magnitude of the wake expansion in the freestream computation was similar to the experimental wake expansion observed with $6.3 \%$ and $9.9 \%$ blockage.

Following the identification of testing practices related to blockage. the effect of the Reynolds number on the development of the initial wake expansion was investigated using two different rotors. The wake expansion downstream of a $25 \mathrm{~cm}$ diameter, three-bladed MAAE wind turbine became less sensitive to the Reynolds number above a Reynolds number of 20,000 . This behaviour may be related to the laminar-to-turbulent transition behaviour of the E387 airfoil on the rotor blades. The wake downstream of the geometrically-scaled rotor was found to be $40 \%$ to $60 \%$ narrower than the initial wake expansion downstream of the corresponding medium-scale rotor. The work identified the need to develop a wind turbine design for a particular Reynolds number regime as opposed to merely geometrically-scaling a turbine.

The performance of scaled wind farm configurations was then evaluated using $20 \mathrm{~cm}$ diameter MAAE wind turbines installed in the $1.68 \mathrm{~m} \times 1.12 \mathrm{~m}$ atmospheric boundary layer wind tunnel at Carleton University. A scaled boundary layer was generated using triangular boundary layer spires and roughness elements installed along the upstream fetch of the tunnel. Each wind turbine was outfitted with a DC generator and the power output generated by the scaled turbines was used to characterise their performance. A singlenormal hot-wire probe was used to determine the mean speed profiles in the flowfield. Two laterally-aligned wind turbines were separated by a gap and it was observed that when the gap was less than 3 diameters (D). the speed of the flow between the rotors was increased from the rotor plane to approximately $2.5 \mathrm{D}$ downstream. This behaviour was identified as an in-field blockage effect and is analogous to the increase in wind speed caused by blockage in a closed test section. The increased flow speed was associated with a narrowing 
of the wake between the closely-spaced rotors and the concept of capitalising on this in-field blockage effect using a third. offset rotor was investigated. Performance measurements were conducted using 3 gap widths between the outer two turbines and a third, central turbine was placed at 9 different downstream positions. The middle turbine experienced an increase in power when placed within $2.5 \mathrm{D}$ of the upstream rotor plane due to the increase in speed in this region. This approach to planning wind farms will help to limit power losses due to downstream wake effects while providing an increase in power output at mean annual wind speeds. 


\section{Acknowledgements}

It's difficult to believe that 4.5 years of work have gone into this $\mathrm{PhD}$ and that I've turned the page on 12 years at Carleton. I value all of the relationships and friendships that have been developed throughout that tenure and I have many people to thank. I'm very grateful for the help provided by my supervisors, Daniel Feszty and Fred Nitzsche over the past 6.5 years. Thank you for believing in me and for allowing me the freedom to find my way through this degree. This thesis could not have taken place without the research, equipment, and travel funding from my supervisors, from the Department of Mechanical and Aerospace Engineering, and from Carleton. I'm also truly grateful to the Department for allowing me to use the atmospheric boundary layer wind tunnel for so many hours, despite the low frequency noise and vibrations felt in the departmental office. A large portion of my graduate degrees were funded by OGS, NSERC. and MITACS. Thank you for providing me with the opportunity to focus on my studies and please continue to promote and to fund science and engineering research in our public institutions so that future students may have the same opportunities.

I am very grateful for patience, support. and help of Alex Proctor and Kevin Sangster in the machine shop. The technologists in the department, Stephan Biljan, Steve Truttman, David Raude, and Gary Clements, all played a very important role in identifying and resolving design challenges along the way. Stephan was always willing to share the wind 
tunnel and provided help and support for all of the rapid prototyping that took place throughout my PhD. A special mention has to go to David Raude for his help assembling and wiring the scaled wind farm. setting up the data acquisition card. and for fixing a very broken roughness panel in the wind tunnel. I also need to thank Mario Mongeon and the maintenance department at Carleton for having the wind tunnel operational again after only two days when some very old fuses blew. Thank you to Nancy Powell. Christie Egbert, Irene Helder, Nermin Ibrahim, and Marlene Groves. for their help with bookings, purchases, cheque requisitions, scholarship applications, and for helping me navigate through Carleton for the past 12 years. The wonderful staff in the Library and at Interlibrary Loans have tracked down dozens of articles for me over the past 4 years and are also owed a very big thank you.

I've been advised and supported in my work by a number of individuals. including Edgar Matida, Metin Yaras, Joana Rocha, and Alanna Wall. Their input and advice was indispensable! I need to thank Gord MacIsaac for his help setting up and troubleshooting hot-wire equipment and for his willingness to work around each other's testing schedules in the water channel lab. Hristo Valtchanov also helped to get me up and running in the water channel. Thank you to Jim Robinson, a long time colleague, who offered to take high quality photos of the wind turbines. To Joshua Brinkerhoff, my colleague, friend, and office-mate - I wish you the best of luck in what will undoubtedly be a very successful career and I look forward to having the opportunity to work together in the future.

Thank you to my dearest friend Sean Dauphinee, my brother Colin, the Gillich family, my curling team. and all of the friends and family that provided the encouragement and support needed to make it through this very long undertaking. I've been very fortunate to have had a series of incredible roommates throughout this degree and would like to thank Peter Gillich, Natalia Jaworska, and Stéphanie Brodeur for helping to make home the best place to be. My parents. John and Donna McTavish. have always been a steadying. positive 
influence in my life and I'm very thankful for their love and support. I hope to see much more of them in their well-earned retirement!

And finally, I need to thank my partner. Josée Rivard. Her dedication and passion as a teacher and educator has been an inspiration to me. The research and writing involved in this thesis simply could not have taken place without her love, understanding. and kindness. and I am so fortunate to be able to share this life with her. 


\section{Table of Contents}

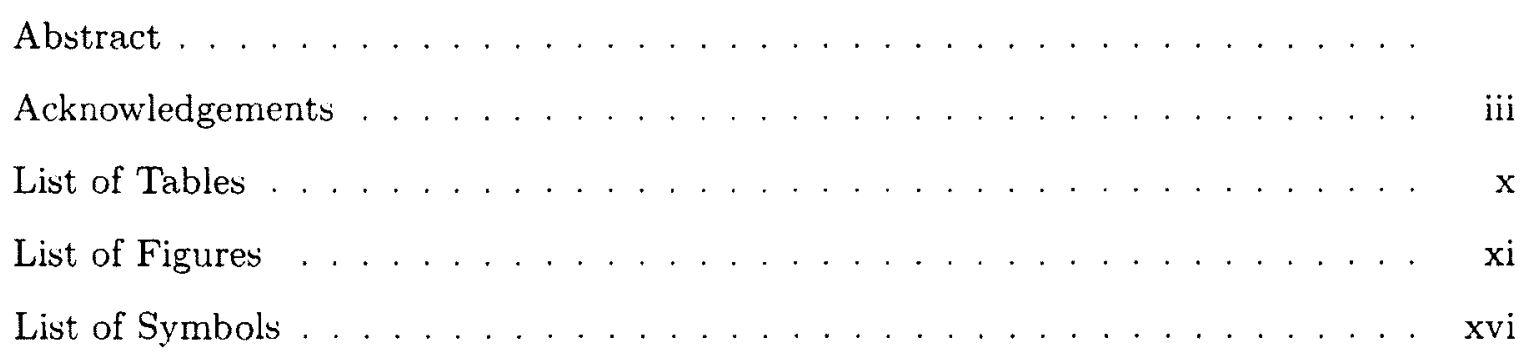

1 Introduction $\quad 1$

1.1 Wind Energy Policy and Wind Farm Development in Ontario . . . . . . . 2

1.2 Motivation - Improving the Performance of Wind Farms . . . . . . . . . 5

1.3 Objectives . . . . . . . . . . . . . . . . . . . . 12

1.4 Approach . . . . . . . . . . . . . . . . . . . 13

1.5 Structure of the Thesis . . . . . . . . . . . . . . . 15

2 Background and Literature Review $\quad 17$

2.1 Wind Turbine Aerodynamics . . . . . . . . . . . . . . . . . . . 17

2.1 .1 Blade Aerodynamics . . . . . . . . . . . . . 18

2.1 .2 The Atmospheric Boundary Layer . . . . . . . . . . . . . . 21

2.1 .3 Wake Development . . . . . . . . . . . . . . . 23

2.1.4 Wind Farm Performance . . . . . . . . . . . . . . 27

2.2 Review of Existing Experiments . . . . . . . . . . . . . . 28

2.2 .1 Single Rotor Experiments . . . . . . . . . . . . . . . . 32

2.2 .2 Blockage Effects . . . . . . . . . . . . . 37 
2.2 .3 Reynolds and scaling . . . . . . . . . . . . . . . 40

2.2.4 Wake Interference Effects . . . . . . . . . . . . . . 43

2.2 .5 Scaled wind farms . . . . . . . . . . . . . . . . 46

2.2.6 Increasing wind farm performance . . . . . . . . . . . 49

2.3 Computational Approaches . . . . . . . . . . . . . . . . . 50

2.3.1 Blade Element Momentum Theory . . . . . . . . . . . . . . . 51

2.3.2 Wind Turbine Wake Modeling . . . . . . . . . . . . 51

2.3.3 Prescribed- and Free-wake Methods . . . . . . . . . . . 53

2.3.4 Navier-Stokes Computational Fluid Dynamics . . . . . . . . . 54

2.3.4.1 Actuator Line Techniques . . . . . . . . . . . . 57

2.3 .5 Hybrid CFD-Vortex Methods . . . . . . . . . . . . . 59

3 Experimental and Computational Methods $\quad 61$

3.1 Scaled wind turbines . . . . . . . . . . . . . . . . . . 61

3.1 .1 MAAE rotor .......................... 62

3.1 .2 Scaled TU Delft turbine . . . . . . . . . . . . . . . . . . . . 69

3.2 Water channel experiments . . . . . . . . . . . . . . . . 70

3.2.1 Quantitative dye visualisation and tip vortex identification .... 74

3.2 Rotor control . . . . . . . . . . . . . . . . 76

3.2 .3 Scale measurements . . . . . . . . . . . . . . . . . 77

3.2.3.1 Balance calibrations . . . . . . . . . . . . 79

3.2.4 Blockage corrections and free surface effects . . . . . . . . . . 82

3.3 Wind tunnel experiments $\ldots \ldots \ldots \ldots$

3.3.1 Atmospheric boundary layer development . . . . . . . . . . . . . . 92

3.3 .2 Wind turbines . . . . . . . . . . . . . . . . 96

3.3.3 Instrumentation and data acquisition system . . . . . . . . . 99

3.3 .4 Traverse design . . . . . . . . . . . . . . . . . . 103

3.3 .5 Hot-wire measurements . . . . . . . . . . . . . . . 105

3.3.5.1 Calibrations and temperature correction . . . . . . . 107

3.3.5.2 Data reduction . . . . . . . . . . . . . . 112 
3.4 Computational methods . . . . . . . . . . . . . . . . 113

3.4 .1 GENUVP . . . . . . . . . . . . . . . . 114

3.4.1.1 Validation ..................... 115

4 Evaluating blockage effects on wind turbine wake development $\quad 120$

4.1 Assessment of the experimental setup . . . . . . . . . . . . 121

4.1.1 Effect of the dye setting on the initial wake expansion . . . . . . 122

4.1.2 Effect of the dye setting on the measured forces . . . . . . . . . . 125

4.1.3 Effect of isolating the turbine strut from the wake . . . . . . . 126

4.1.4 Summary of the developed testing guidelines . . . . . . . . . . . 128

4.2 Wake development . . . . . . . . . . . . . . . . 128

4.3 Effects of blockage on the initial wake expansion . . . . . . . . . . 133

4.3 .1 Sensitivity to the streakline pattern . . . . . . . . . 138

4.3.2 Comparison with computational results . . . . . . . . . 140

4.4 Effect of blockage on thrust forces . . . . . . . . . . . . . 142

4.4 .1 Corrected Force Coefficients . . . . . . . . . . . . . . 142

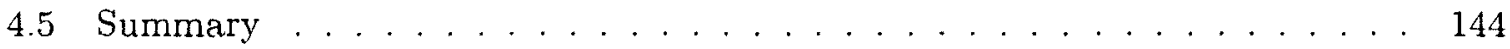

5 Evaluating Reynolds number effects in scaled wind turbine experiments 147

5.1 Scaled rotors . . . . . . . . . . . . . . . . . . . . . 148

5.2 Test conditions . . . . . . . . . . . . . . . . . . . 149

5.2 .1 MAAE test conditions . . . . . . . . . . . . . 150

5.2 .2 Scaled TU Delft test conditions . . . . . . . . . . . . . . 150

5.3 Description of the flowfields . . . . . . . . . . . . . . 150

5.3 .1 MAAE flowfield ........................ 151

5.3 .2 Scaled TU Delft flowfield . . . . . . . . . . . . . . 151

5.4 Reynolds number effects on the wake expansion . . . . . . . . . . . 154

5.4 .1 MAAE wake expansion ..................... 154

5.4 .2 Scaled TU Delft wake expansion . . . . . . . . . . . 156

5.5 Effect of the Reynolds number on the thrust coefficient . . . . . . . . 162 
5.5.1 MAAE thrust coefficients - water channel . . . . . . . . . 163

5.5 .2 MAAE thrust coefficients - wind tunnel . . . . . . . . 163

5.5 .3 Scaled TU Delft thrust coefficients . . . . . . . . . . . . 165

5.5 .4 Comparison with other results .............. 167

5.6 Summary . . . . . . . . . . . . . . . . . . . . 167

6 The performance of wind farms with closely-spaced wind turbines $\quad 170$

6.1 Atmospheric boundary layer characteristics . . . . . . . . . . . . 171

6.1 .1 Flow asymmetry . . . . . . . . . . . . . . . 172

6.2 Single turbine results . . . . . . . . . . . . . . . . . . 173

6.3 Wake losses in longitudinal configurations . . . . . . . . . . . . . . 182

6.4 Generating in-field blockage with closely-spaced rotors . . . . . . . . . . 186

6.5 Power augmentation in three-rotor configurations . . . . . . . . . . 193

6.5 .1 Performance in a rough boundary layer . . . . . . . . . . 198

6.5.2 Performance in a smooth boundary layer . . . . . . . . . . . . 202

6.5 .3 Downstream effects . . . . . . . . . . . . . . . 203

6.5 .4 Off-design conditions . . . . . . . . . . . . . 204

6.5.5 Cumulative power increase . . . . . . . . . . . . 207

6.6 Summary . . . . . . . . . . . . . . . . . . . 208

7 Conclusions, Academic Contributions, and Future Work 210

7.1 Conclusions . . . . . . . . . . . . . . . . . . . 212

7.1.1 Blockage effects . . . . . . . . . . . . . . . . . 212

7.1.2 Reynolds number and scaling effects . . . . . . . . . . . 213

7.1.3 In-field blockage effects in wind farms with closely-spaced wind turbines 214

7.2 Summary of Academic Contributions . . . . . . . . . . . . . . 215

7.3 Recommendations and future work . . . . . . . . . . . . . . 216

$\begin{array}{ll}\text { References } & 219\end{array}$ 


\section{List of Tables}

2.1 A summary of recently-published wind turbine experiments. . . . . . . . . 29

3.1 MAAE Design Parameters. . . . . . . . . . . . . . . . . . . . . . . 69

3.2 Comparison between the full-scale TU Delft rotor and the $1 / 4.8$ scale rotor. 71

3.3 Calibration weights from March 2011 - horizontal orientation. . . . . . . . 80

3.4 Calibration weights from August 2012 - vertical orientation. . . . . . . . . . 83

3.5 Anemometer settings. . . . . . . . . . . . . . . . 107

3.6 Calibration coefficients. . . . . . . . . . . . . . . . 113

3.7 Computational and experimental test conditions. . . . . . . . . . . 117

4.1 Test conditions for the blockage experiments. . . . . . . . . . . . 135

5.1 Test conditions for the MAAE rotor. . . . . . . . . . . . . . 150

5.2 Test conditions for the scaled and medium-scale TU Delft rotor. . . . . . . 151 


\section{List of Figures}

1.1 Wind energy capacity in Ontario from 2002-2016. . . . . . . . . . . 3

1.2 Wind farm map of central, southern. and eastern Ontario. . . . . . . . . 4

1.3 Monthly wind energy production in Ontario since 2006. . . . . . . . . . . . 5

1.4 Schematic of wake interference effects. . . . . . . . . . . . . . 6

1.5 Layout and power output of the offshore Horns Rev I wind farm in Denmark. 7

1.6 Layout of the Talbot wind farm. . . . . . . . . . . . . . . . . . . 9

1.7 Mean wind speed distribution in Ontario at a height of $80 \mathrm{~m} . \ldots 10$

1.8 Schematic of a typical power curve. . . . . . . . . . . . . . . . 11

1.9 Annual capacity factors for 14 wind farms in Ontario. . . . . . . . . . . 12

2.1 Loads generated by a wind turbine rotor. . . . . . . . . . . . . . . 19

2.2 Regulating power output using stall control or pitch control. . . . . . . . . . 22

2.3 Schematic of the atmospheric boundary layer. . . . . . . . . . . . 23

2.4 Description of wind turbine wake evolution. . . . . . . . . . . . . . . . 24

2.5 Representation of tip vortex shedding. . . . . . . . . . . . . . 26

2.6 Schematic of the velocity and turbulence intensity profiles. . . . . . . . . 27

2.7 Distribution of blockage in existing experiments. . . . . . . . . . . . . 38

2.8 Summary of the Reynolds numbers in existing experiments. . . . . . . . . . 42

3.1 Solid model of the scaled MAAE wind turbine. . . . . . . . . . . . . . . . 62

3.2 Comparison of the E387 airfoil with a typical wind turbine airfoil. . . . . 66 
3.3 Eppler E387 lift curves at low Reynolds numbers. . . . . . . . . . . . . . 67

3.4 The chord and twist distribution of the scaled MAAE rotor blades. . . . . . 68

3.5 Solid model of the scaled TUDelft rotor . . . . . . . . . . . . . . . . 70

3.6 The RHRC 2436 water channel. . . . . . . . . . . . . . . . . . 72

3.7 The pressurised dye delivery system. . . . . . . . . . . . . . . 72

3.8 The RHRC 2436 water channel at Carleton University. . . . . . . . . . . 73

3.9 Schematic of the wake expansion and the tip vortex pitch. . . . . . . 75

3.10 Parallax correction schematic. . . . . . . . . . . . . . 75

3.11 The HE6X6 scale and components. . . . . . . . . . . . . . . . 77

3.12 Schematic of the scale coordinates. . . . . . . . . . . . . 78

3.13 Vertical scale arrangement. . . . . . . . . . . . . 78

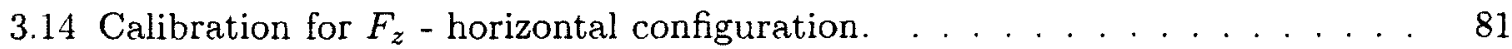

3.15 Position sensitivity of $F_{z}$ - horizontal configuration. . . . . . . . 81

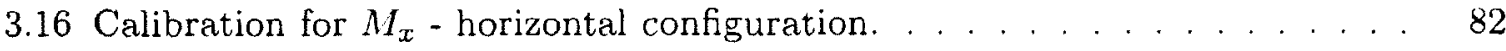

3.17 Schematic of the vertical calibration setup. . . . . . . . . . . . 83

3.18 Calibration for $M_{x}$ - vertical orientation. . . . . . . . . . 84

3.19 Schematic of the actuator disc-based blockage correction. . . . . . . . . 85

3.20 Schematic of the free surface variation due to blockage. . . . . . . . 88

3.21 The open-circuit ABL tunnel at Carleton University. . . . . . . . . . . 89

3.22 Scaled model of Montreal's Olympic Stadium. . . . . . . . . . . . . . . 90

3.23 Drawing of the ABL wind tunnel room. . . . . . . . . . . . . . 91

3.24 Layout of the wind turbine positions. . . . . . . . . . . . . . . . 93

3.25 Flow conditioning elements in the wind tunnel. . . . . . . . . . . . 94

3.26 Wind turbine model in the wind tunnel. . . . . . . . . . . . . . . 97

3.27 Photograph of the primary instrumentation and equipment. . . . . . . . 99

3.28 The NI Compaq-DAQ system and wiring diagram. . . . . . . . . . 100

3.29 Calibration curve for the PX653 pressure transducer. . . . . . . . . . . 101

3.30 Manual calibration curve for the string potentiometer. . . . . . . . . . 102 
3.31 Photograph of the traverse equipment. . . . . . . . . . . . 104

3.32 Wiring diagram for the traverse system. . . . . . . . . . . . . . . 105

3.33 Calibration curves for determining the position of the hot-wire probe. . . 106

3.34 Calibration setup in the wind tunnel. . . . . . . . . . . . . 108

3.35 Calibration curve developed at low and high velocities. . . . . . . . 110

3.36 Sensitivity of the calibration curve to changes in temperature. . . . . . . 111

3.37 Long term calibration stability at $23.0^{\circ} \mathrm{C} \ldots \ldots \ldots \ldots$

3.38 The NREL Phase VI UAE turbine. . . . . . . . . . . . . . . . . 116

3.39 Comparison of the computational wake profiles. . . . . . . . . . . 118

3.40 Validation of the axial and radial tip vortex locations. . . . . . . . . . 119

4.1 Tip vortex visualisation at different dye settings. . . . . . . . . . . . 123

4.2 Initial wake expansion as a function of the dye setting. . . . . . . . . . . 124

4.3 Deviation of the lateral vortex core locations. . . . . . . . . . . . . 125

4.4 Effect of the dye setting on $C_{T, U} \ldots \ldots \ldots \ldots \ldots$

4.5 Effect of isolating the strut on $C_{T, U} \ldots \ldots \ldots \ldots \ldots$

4.6 Tip vortex visualisation downstream of a $25 \mathrm{~cm}$ rotor at $\lambda=6.1 . \ldots 130$

4.7 Merged tip vortices in the near wake. . . . . . . . . . . . . . 131

4.8 Computationally-predicted tip vortex structures. . . . . . . . . . . 132

4.9 Scaled MAAE turbine blades. . . . . . . . . . . . . . . . . . . . 134

4.10 Initial wake expansion as a function of blockage. . . . . . . . . . 136

4.11 Initial wake expansion variation with blade number. . . . . . . . . 139

4.12 Comparison between the experimental and computational wake expansion. 141

4.13 Uncorrected and corrected thrust coefficients at $R e_{t i p} \approx 23.000$ and $\lambda \approx 6 . \quad 143$

4.14 Relationship between blockage, $\Delta C_{T}$, and the wake expansion. . . . . . 144

$5.125 \mathrm{~cm}$ diameter MAAE and TU Delft rotor blades. . . . . . . . . . . . 149

5.2 Tip vortex visualisation at several Reynolds numbers. . . . . . . . . . . 152

5.3 Scaled TU Delft tip vortex visualisation. . . . . . . . . . . . 153 
5.4 Merged tip vortices in the scaled TU Delft near wake. . . . . . . . . . . 154

5.5 Wake expansion dependence on $R e_{t i p}-$ MAAE . . . . . . . . . . 155

5.6 Initial wake expansion at $\lambda \approx 6$ and $\theta_{\text {tip }}=4^{\circ} \ldots \ldots \ldots 158$

5.7 Initial wake expansion at $\lambda \approx 8$ and $\theta_{t i p}=4^{\circ} \ldots \ldots \ldots 159$

5.8 Initial wake expansion at $\lambda \approx 8$ and $\theta_{t i p}=0^{\circ} \ldots \ldots \ldots 159$

5.9 Comparison of the small-scale tip vortex pitch with medium-scale results. . 162

5.10 Relationship between the $R e_{t i p}, C_{T}$. and wake expansion - MAAE. . . . 164

5.11 Schematic of the upstream test section in the ABL tunnel. . . . . . . . . 165

5.12 Comparison of wind tunnel and water channel-derived $C_{T, C}-$ MAAE. $\ldots 165$

5.13 Reynolds number and tip pitch effects on $C_{T}$ - Scaled TU Delft. . . . . 166

5.14 Compilation of $C_{T}$ values from experiments in literature. . . . . . . . . 168

6.1 Velocity and turbulence intensity profiles at $X=0$ and the tunnel centreline. 173

6.2 Schematic of the ABL measurement plane. . . . . . . . . . . . . . . 174

6.3 Normalised speed contours at $X / D=0 \ldots \ldots \ldots \ldots \ldots$

6.4 Schematic of the single rotor HWA measurement window. . . . . . . . . . 176

6.5 Hub height contour plot - Single rotor . . . . . . . . . . . . . . . 177

6.6 Comparison of the wake expansion profile for a single rotor. . . . . . . . . . 178

6.7 Wake speed validation downstream of a single rotor. . . . . . . . . . . 180

6.8 Power output variation in the wind tunnel. . . . . . . . . . . . . . 181

6.9 Longitudinally-aligned wind turbines. . . . . . . . . . . . . . . . . . 182

6.10 The power output of a downstream longitudinally-separated rotor. . . . . . 183

6.11 Comparison of longitudinally-aligned results. . . . . . . . . . . . . . . . 184

6.12 The longitudinally-aligned $3 \times 3$ wind farm. . . . . . . . . . . . 185

6.13 Power results in the $3 \times 3$ wind farm. . . . . . . . . . . . . 186

6.14 Comparison of wind farm results . . . . . . . . . . . . . . . . . . 187

6.15 Schematic of the two-rotor arrangement and the HWA measurement plane. 188

6.16 Normalised speed contours at hub height. . . . . . . . . . . . . . . 190

6.17 Normalised centreline speed at several gap widths. . . . . . . . . . . 193 
6.18 Wake profile as a function of the gap width. . . . . . . . . . . . 194

6.19 Schematic of the gap in three-rotor configurations. . . . . . . . . . . . 194

6.20 Speed contours and relative power output for three lateral turbines. . . . . 195

6.21 Velocity contours and relative power output $-G=2 \mathrm{D}, S_{t i p}=0.5 \mathrm{D} \ldots \ldots 6$

6.22 Repeatability of speed measurements. . . . . . . . . . . . . . . . 197

6.23 Velocity contours and relative power output $-G=2 D . S_{t i p}=0.5 D \ldots 198$

6.24 Closely-spaced wind farm performance in a rough boundary layer. . . . . . 200

6.25 Closely-spaced wind farm performance in a smooth boundary layer. . . . . 203

6.26 Effect of the gap on the output of a rotor located 6.5D downstream. . . . 204

6.27 Schematic of three turbines with $S_{t i p}=0.5 \mathrm{D}$ in yawed configurations. . . . 205

6.28 Power output as a function of the wind angle. . . . . . . . . . . . 206

6.29 Cumulative change in the power output in a rough boundary layer. . . . . . 207

6.30 Cumulative change in the power output as a function of the boundary layer. 208 


\section{List of Symbols}

\section{Symbols}

\begin{tabular}{|c|c|c|}
\hline$a$ & Axial induction factor & \\
\hline$A, B, C, D, E$ & Calibration coefficients & \\
\hline$A_{\text {blades }}$ & Frontal rotor blade area & {$\left[\mathrm{m}^{2}\right]$} \\
\hline$A_{\text {swept }}$ & Rotor swept area & {$\left[\mathrm{m}^{2}\right]$} \\
\hline$A_{\text {: }}$ & Cross-sectional area & {$\left[\mathrm{m}^{2}\right]$} \\
\hline$\alpha$ & Angle of attack, power law coefficient & {$[\operatorname{deg}],[-]$} \\
\hline$\alpha_{s t a l l}$ & Stall angle & [deg] \\
\hline$B_{r}$ & Blockage ratio & \\
\hline$\beta$ & Twist angle & [deg] \\
\hline$c$ & Chord, constant & {$[\mathrm{m}],[-]$} \\
\hline$c_{t i p}$ & Blade tip chord & {$[\mathrm{m}]$} \\
\hline$C_{f}$ & Friction coefficient & \\
\hline$C_{L \max }$ & Maximum lift coefficient & \\
\hline$C_{P}$ & Power coefficient & \\
\hline$C_{Q}$ & Torque coefficient & \\
\hline$C_{T}$ & Thrust coefficient & \\
\hline$C_{T, C}$ & Corrected thrust coefficient & \\
\hline$C_{T, U}$ & Uncorrected thrust coefficient & \\
\hline$d$ & Corrected distance, moment arm, constant & {$[\mathrm{m}]$} \\
\hline$d_{s p}$ & String potentiometer distance & {$[\mathrm{m}]$} \\
\hline$d_{V}$ & Vernier scale distance & {$[\mathrm{ft}]$} \\
\hline$D$ & Diameter, roughness element spacing & {$[\mathrm{m}]$} \\
\hline$\delta$ & Reference height & {$[\mathrm{m}]$} \\
\hline$\Delta P$ & Change in power & {$[\%]$} \\
\hline$\epsilon_{B}$ & Blockage factor & \\
\hline$\eta_{a r r a y}$ & Array efficiency & \\
\hline$F$ & Correction factor & \\
\hline$F r$ & Froude number & \\
\hline
\end{tabular}




\begin{tabular}{|c|c|c|}
\hline$F_{s}$ & Scaling factor & \\
\hline$F_{T a t t}$ & Tangential force & {$[N]$} \\
\hline$F_{\text {Thrust }}$ & Thrust force & {$[N]$} \\
\hline$F_{\varsigma}$ & Force in the $\mathrm{x}$-direction & {$[N]$} \\
\hline$F_{z}$ & Force in the z-direction & {$[N]$} \\
\hline$\varphi$ & Azimuth angle & [deg] \\
\hline$g$ & Gravity & {$\left[\mathrm{m} / \mathrm{s}^{2}\right]$} \\
\hline$G$ & Gap width & {$[\mathrm{m}]$} \\
\hline$\gamma$ & Wind angle [deg] & \\
\hline$h$ & Plane to grid distance, spire height & {$[\mathrm{m}]$} \\
\hline$H$ & Camera to grid distance, tunnel height & {$[\mathrm{m}]$} \\
\hline$k$ & Roughness element height & {$[\mathrm{m}]$} \\
\hline$L$ & Photographed grid distance & {$[\mathrm{m}]$} \\
\hline$\lambda$ & Tip speed ratio & \\
\hline$M_{x}$ & Moment about the $x$-axis & {$[\mathrm{Nm}]$} \\
\hline$N$ & Number of turbines & \\
\hline$\nu_{\text {water }}$ & kinematic viscosity of water & {$\left[\mathrm{m}^{2} / \mathrm{s}\right]$} \\
\hline$p$ & Blockage parameter & \\
\hline$P$ & Power & {$[\mathrm{W}]$} \\
\hline$P_{\text {elec }}$ & Electrical power & {$[\mathrm{W}]$} \\
\hline$P_{\text {meas }}$ & Measured power & {$[W]$} \\
\hline$P_{\text {single }}$ & Power from an isolated turbine & {$[\mathrm{W}]$} \\
\hline$q$ & Blockage parameter & \\
\hline$Q$ & Torque & {$[\mathrm{Nm}]$} \\
\hline$r$ & Local radius & {$[\mathrm{m}]$} \\
\hline$R$ & Radius & {$[\mathrm{m}]$} \\
\hline$R_{c}$ & Commercial-scale radius & {$[\mathrm{m}]$} \\
\hline$R_{m}$ & Scaled model radius & {$[\mathrm{m}]$} \\
\hline$R e_{D}$ & Reynolds number based on $D$ & \\
\hline$R e_{t i p}$ & Tip Reynolds number & \\
\hline$\rho$ & Fluid density & {$\left[\mathrm{kg} / \mathrm{m}^{3}\right]$} \\
\hline$s$ & Wall tip clearance & {$[\mathrm{m}]$} \\
\hline$S_{t i p}$ & Blade tip separation distance & {$[\mathrm{m}]$} \\
\hline$T u$ & Turbulence intensity & {$[\%]$} \\
\hline$\theta_{t i p}$ & Tip pitch angle & [deg] \\
\hline$U$ & Local speed & {$[\mathrm{m} / \mathrm{s}]$} \\
\hline$U_{b}$ & Bypass speed & {$[\mathrm{m} / \mathrm{s}]$} \\
\hline$U_{l u b}$ & Speed at hub height & {$[\mathrm{m} / \mathrm{s}]$} \\
\hline$U_{r \in f}$ & Speed at hub height & {$[\mathrm{m} / \mathrm{s}]$} \\
\hline$U_{r m s}$ & Root mean square of speed & {$[\mathrm{m} / \mathrm{s}]$} \\
\hline$U_{t i p}$ & Blade tip speed & {$[\mathrm{m} / \mathrm{s}]$} \\
\hline$U_{D}$ & Speed at the rotor disc plane & {$[\mathrm{m} / \mathrm{s}]$} \\
\hline
\end{tabular}




$\begin{array}{lll}U_{F} & \text { Speed in freestream conditions } & {[\mathrm{m} / \mathrm{s}]} \\ U_{T} & \text { Speed in tunnel conditions } & {[\mathrm{m} / \mathrm{s}]} \\ U_{1}, U_{2}, U_{3} & \text { Blockage correction velocities } & {[\mathrm{m} / \mathrm{s}]} \\ U_{\delta} & \text { Speed at a reference height } & {[\mathrm{m} / \mathrm{s}]} \\ U_{\infty} & \text { Freestream speed } & {[\mathrm{m} / \mathrm{s}]} \\ V & \text { Voltage } & {[\mathrm{V}]} \\ V_{a m p} & \text { Amplifier output voltage } & {[\mathrm{V}]} \\ V_{\text {out }, s p} & \text { String potentiometer voltage } & {[\mathrm{V}]} \\ V_{r o t} & \text { Local speed due to rotation } & {[\mathrm{m} / \mathrm{s}]} \\ V_{R} & \text { Resultant velocity } & {[\mathrm{m} / \mathrm{s}]} \\ \omega & \text { Rotational speed } & {[\mathrm{rad} / \mathrm{s}]} \\ \Omega & \text { Resistance } & {[\Omega]} \\ \Omega_{\mathrm{c}} & \text { Commercial-scale rotational speed } & {[\mathrm{rad} / \mathrm{s}]} \\ \Omega_{m} & \text { Scaled model rotational speed } & {[\mathrm{rad} / \mathrm{s}]} \\ x & \text { Axial distance from tip } & {[\mathrm{m}]} \\ X & \text { Downstream axial location } & {[\mathrm{m}]} \\ y & \text { Lateral position from tip } & {[\mathrm{m}]} \\ Y & \text { Lateral position } & {[\mathrm{m}]} \\ z & \text { Water depth } & {[\mathrm{m}]} \\ Z & \text { Vertical position } & {[\mathrm{m}]}\end{array}$

\section{Acronyms}

$\begin{array}{ll}\text { ABL } & \text { Atmospheric Boundary Layer } \\ \text { ADV } & \text { Acoustic Doppler Velocimetry } \\ \text { BEMT } & \text { Blade Element Momentum Theory } \\ \text { BVI } & \text { Blade Vortex Interaction } \\ \text { CFD } & \text { Computational Fluid Dynamics } \\ \text { DAQ } & \text { Data Acquisition System } \\ \text { DTU } & \text { Technical University of Denmark } \\ \text { ECN } & \text { Energy Center of the Netherlands } \\ \text { FIT } & \text { Feed-in Tariff } \\ \text { GENUVP } & \text { Generalized Unsteady Vortex Particle Method } \\ \text { HWA } & \text { Hot-wire Anemometry } \\ \text { LES } & \text { Large Eddy Simulation } \\ \text { MAAE } & \text { Mechanical and Aerospace Engineering } \\ \text { MAV } & \text { Micro Air Vehicle } \\ \text { MEXICO } & \text { Modern Experiments in Controlled Conditions } \\ \text { NI } & \text { National Instruments } \\ \text { NREL } & \text { National Renewable Energy Laboratory } \\ \text { PIV } & \text { Particle Image Velocimetry }\end{array}$




$\begin{array}{ll}\text { RANS } & \text { Reynolds-Averaged Navier-Stokes } \\ \text { RESOP } & \text { Renewable Energy Standing Offer Program } \\ \text { RFP } & \text { Request for Proposals } \\ \text { RHRC } & \text { Rolling Hills Research Corporation } \\ \text { TU Delft } & \text { Delft University of Technology } \\ \text { UAE } & \text { Unsteady Aerodynamics Experiment } \\ \text { UAV } & \text { Uninhabited Air Vehicle } \\ \text { UTM } & \text { Universal Transverse Mercator } \\ \text { VAWT } & \text { Vertical Axis Wind Turbine } \\ \text { VPM } & \text { Vortex Particle Method } \\ \text { VTM } & \text { Vorticity Transport Method }\end{array}$




\section{Chapter 1}

\section{Introduction}

The prediction of the power output and performance of wind turbines is a research area that has seen growing interest recently as the demand for greater renewable energy production increases. The current worldwide installed wind turbine capacity is $273 \mathrm{GW}$. with installed capacity concentrated in China. the United States. Western Europe, and India [1]. Canada currently has an installed wind turbine capacity of $6,500 \mathrm{MW}$ [2]. While this represents only approximately $2 \%$ of Canadian electrical demand, the industry has seen continued growth of about $25 \%$ per year throughout the past decade and is projected to grow to an installed capacity of $10,000 \mathrm{MW}$ by 2015 because of social and governmental initiatives $[2,3]$. This rapid development has been focused on Ontario, which currently accounts for over one third of Canada's installed wind energy capacity, and has been driven by the goal to reduce carbon dioxide emissions. In Ontario, this is being addressed primarily through conservation, the refurbishment of nuclear reactors, and the replacement of coal generation with solar. small hydro. and wind energy [3]. 


\subsection{Wind Energy Policy and Wind Farm Development in Ontario}

A series of initiatives by the provincial government have led to the very rapid deployment of wind energy in Ontario [3]. The first commercial-scale wind turbine in Ontario was installed near Tiverton in Bruce County in 1995 to conduct cold weather testing. Ontario's first wind farm was subsequently built nearby in 2002 and contained five $1.8 \mathrm{MW}$ turbines. The overall installed wind energy capacity in the province is shown in Figure 1.1 and was less than $15 \mathrm{MW}$ until 2006, when the installations that resulted from several provincial initiatives were commissioned. The Government of Ontario released a request for proposals (RFP) in 2004 for up to $300 \mathrm{MW}$ of renewable energy projects and followed this with a subsequent RFP in 2005 for 1000 additional megawatts. Note that $1000 \mathrm{MW}$ of capacity can be assumed to generate enough electricity for 300,000 homes. These two competitive bidding processes led to the commissioning of the first large wind farms in Ontario from 2006 to 2009 [4]. In order to promote smaller, distributed projects, a renewable energy standing offer program (RESOP) was initiated in 2006 for renewable energy projects smaller than 10 MW. Unlike the previous RFPs, the RESOP guaranteed projects a fixed rate of $0.11 \$ / \mathrm{kWh}$. A third competitive RFP was initiated in 2008 for $500 \mathrm{MW}$ of renewable energy that resulted in five new wind farms commissioned between 2010 and 2011. In 2009, the Government of Ontario passed the Green Energy and Green Economy Act, which superseded the previous initiatives and led to the implementation of a Feed-in Tariff (FIT). The FIT guaranteed wind energy developers a fixed price of $0.135 \$ / \mathrm{kWh}$. with additional bonuses depending on the level of community and aboriginal group ownership in a particular project [5]. The first wind farm to result from the FIT program came online in late 2011 and a large number of projects are scheduled to be commissioned, as observed by the large projected increase in installed wind capacity in 2013 in Figure 1.1. Supported by the rapid level of wind energy 
development since the introduction of the FIT. the program was recently extended and revised [6]. The updated FIT 2.0 program reduced the price paid to wind energy developers to $0.115 \$ / \mathrm{kWh}$ to reflect the declining capital costs associated with wind turbines. Ontario now frequently generates more electricity from wind than coal and future wind farms will effectively replace coal generation in the province when coal is phased out in 2014 [7].

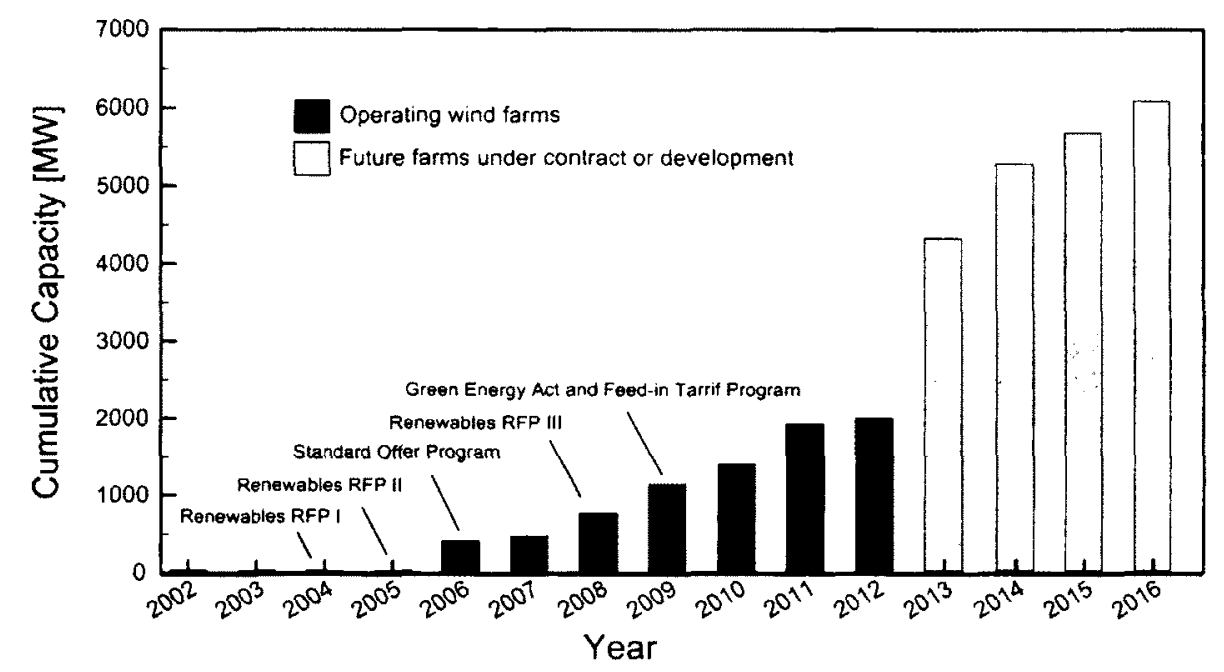

Figure 1.1: Wind energy capacity in Ontario from 2002-2016. Data from $[2.4,7]$.

The majority of Ontario's wind farms have been built in Southern Ontario. Figure 1.2 identifies the locations of wind farms larger than $20 \mathrm{MW}$ that are currently in service as well as those under development and has been modified to only show those wind farms installed in Central, Southern, and Easter Ontario for clarity [7]. The shaded regions in Figure 1.2 indicate the major population centres in Southern Ontario. The significant number of wind farm developments in Southern Ontario results from the high mean annual wind speed in this region and the proximity to existing transmission lines. The majority of future wind farm installations in the province will continue to occur in this region.

While Figure 1.1 represents the installed power capacity in the province. the actual monthly energy production from wind farms in Ontario has been compiled in Figure 1.3 based on hourly data from the Independent Electricity System Operator (IESO) in 


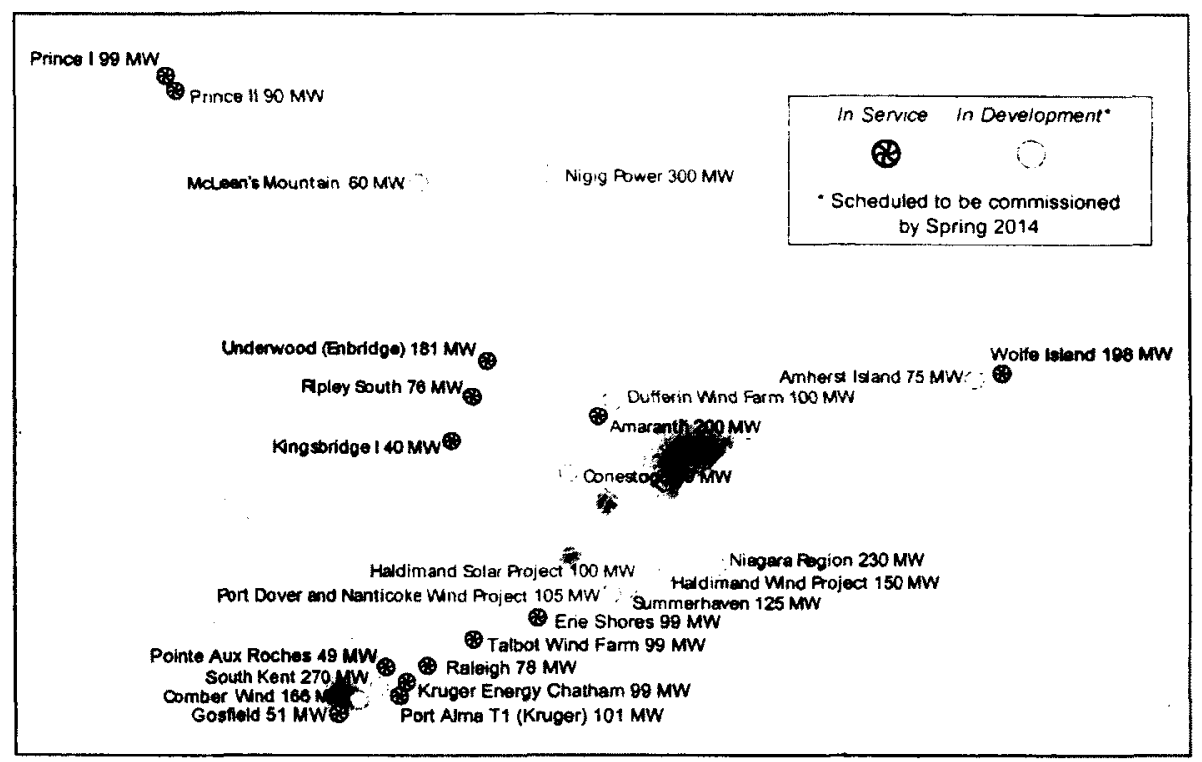

Figure 1.2: Wind farm map of central. southern. and eastern Ontario. Adapted from Ref. [7].

Ontario [7]. The Prince wind farm near Sault Ste Marie was the first large scale wind farm to come online following Ontario's first RFP in 2006 and the largest wind farm in the second RFP was the Wolfe Island wind farm near Kingston. The most recent wind farm to come online is the Pointe aux Roches wind farm in Southern Ontario and this was the first wind farm commissioned within the FIT program. The seasonal variation in wind energy generation in Ontario is also visible in Figure 1.3 and demonstrates that wind energy production is typically maximized between January and May. Despite the seasonal and daily variations in wind energy generation, the province consistently obtains $2 \%$ to $5 \%$ of its energy requirements from wind [7].

The rapid projected growth of wind energy. particularly in Southern Ontario, has led to a number of competing interests and challenges related to environmental considerations, land use, and energy supply. The continued large-scale deployment and integration of wind farms into Ontario's electricity grid will require significant research efforts to optimize wind farm land use and wind farm performance. These two factors will be dependent on the 


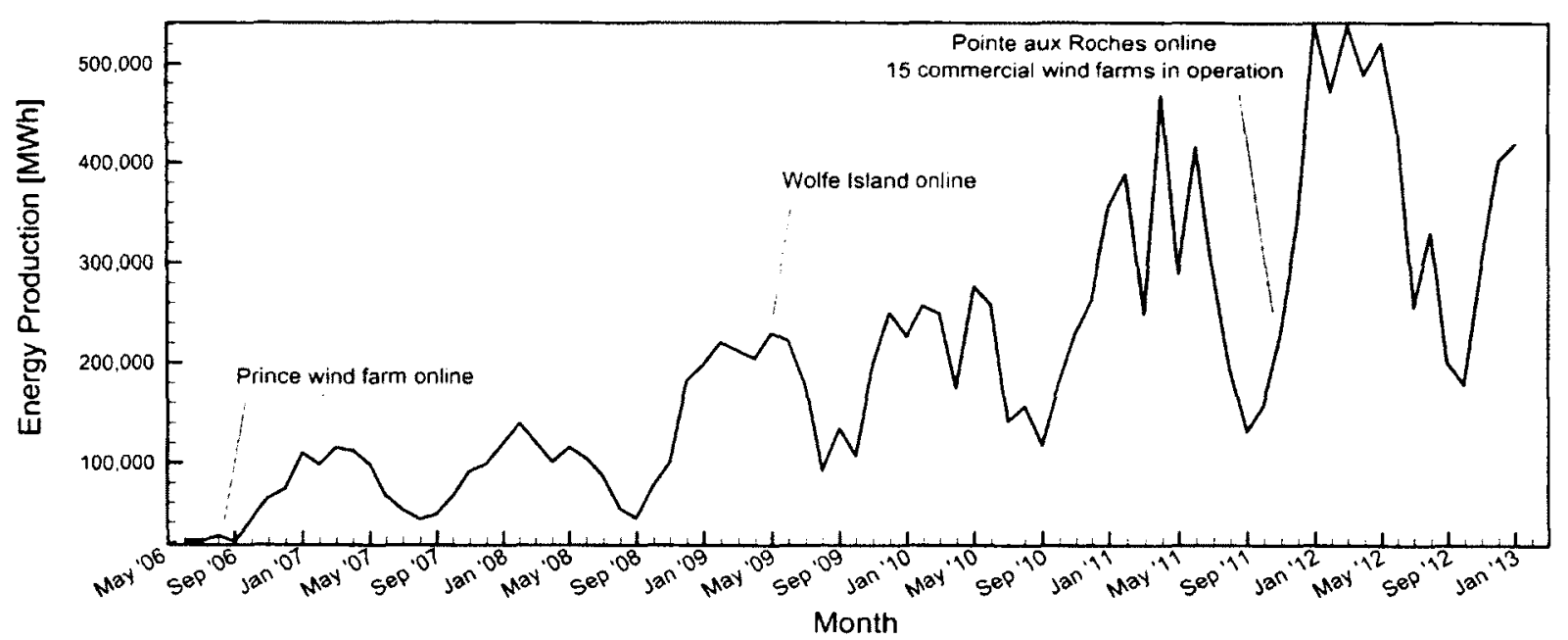

Figure 1.3: Monthly wind energy production in Ontario since 2006. Data from Ref. [7].

development of a thorough understanding of wind turbine wake development and on the identification of novel approaches to designing and planning wind farms.

\subsection{Motivation - Improving the Performance of Wind Farms}

The motivation for the current thesis arises from the need to improve the performance and power generation capabilities of wind farms. This involves characterising and capitalising on both wind turbine wake effects and the local wind speed distribution. Wind farm performance can frequently be degraded due to losses incurred by wake effects. Wind turbines extract energy from the air and, as a result, reduce the wind speed in the wake formed downstream of the rotor. This is illustrated in Figure 1.4 where the upstream wind turbine extracts power from the incident wind $\left(U_{\infty}\right)$. As a result of this phenomenon, the velocity deficit in an upstream turbine wake reduces the power that can be generated by subsequent downstream turbines in a wind farm. The rotor wake width is on the order of the turbine's diameter and the wake expands downstream. The wind speed in the wake will gradually recover to freestream conditions approximately $10-15$ rotor diameters (D) downstream [8,9]. This effect has led to commonly-observed wind farm design 
guidelines. which have established separation distances of $3.5 \mathrm{D}$ to $7 \mathrm{D}$ between turbines in the lateral direction and 7D-10D separation distances between turbines in the longitudinal, or prevailing wind, direction $[8,9]$. As previously-mentioned, the wake will completely recover to the freestream wind speed 10D-15D downstream. but this would impose large separation distances between turbines. The wake speed typically recovers to $80-90 \%$ of the freestream value $7 \mathrm{D}-10 \mathrm{D}$ downstream of a turbine and this has resulted in separation distances that are a compromise between land use and power output.

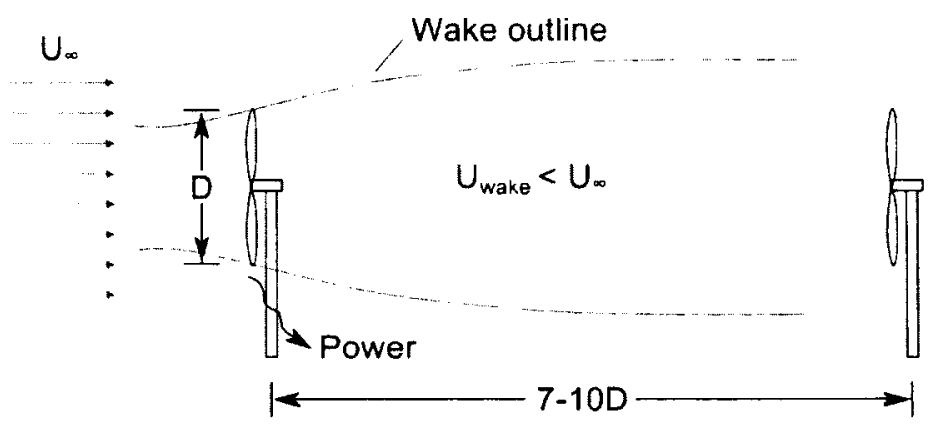

Figure 1.4: Schematic of wake interference effects.

Large offshore wind farms have typically followed these design guidelines and this has resulted in grid-like layouts [10]. The first large offshore wind farm was the Horns Rev I wind farm that was commissioned in 2002 and is located in the North Sea $15 \mathrm{~km}$ west of Denmark. This wind farm consists of 80 wind turbines of $80 \mathrm{~m}$ diameter and $2 \mathrm{MW}$ rated power arranged in an approximate grid with 7D separation between the rotor towers in all directions. The layout of the wind farm can be seen in Figure 1.5(a) and the prevailing wind directions are from the west, south-west, and north-west. The $x$ - and $y$-coordinates in Figure 1.5(a) represent the Universal Transverse Mercator (UTM) coordinates of the turbines. This wind farm has frequently been used as a case study to evaluate wind farm wake effects $[10]$. The power output in a row of turbines can be evaluated by investigating the turbines numbered 1 through 10 indicated in Figure 1.5(a). Two wind directions are indicated in Figure 1.5(a) and present situations where the wind is aligned with the row of turbines (Direction A) and when the wind is at an angle of $15^{\circ}$ to the studied row (Direction 
B), respectively. In both cases. the turbines were assumed to be yawed into the wind and the wakes can be considered to be approximately aligned with the direction of the wind [10].

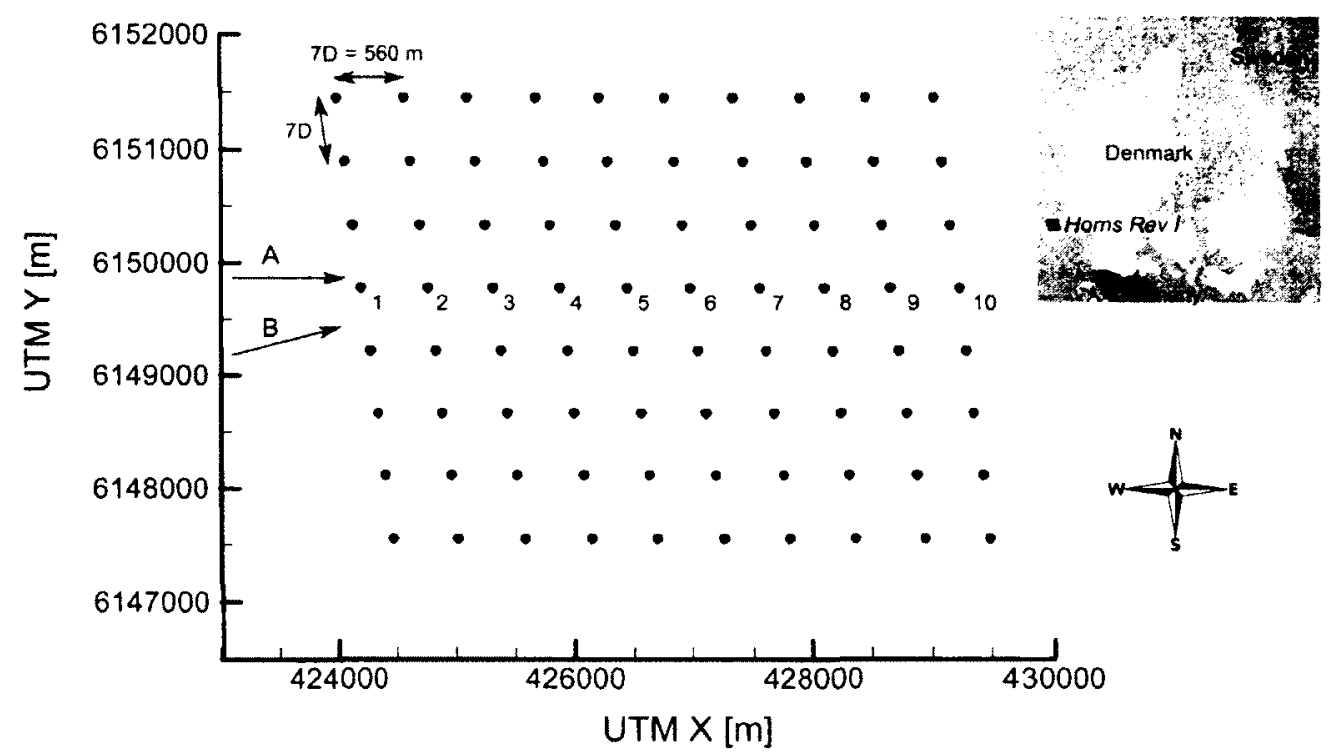

(a) Layout of the Horns Rev I wind farm. Adapted from [10].

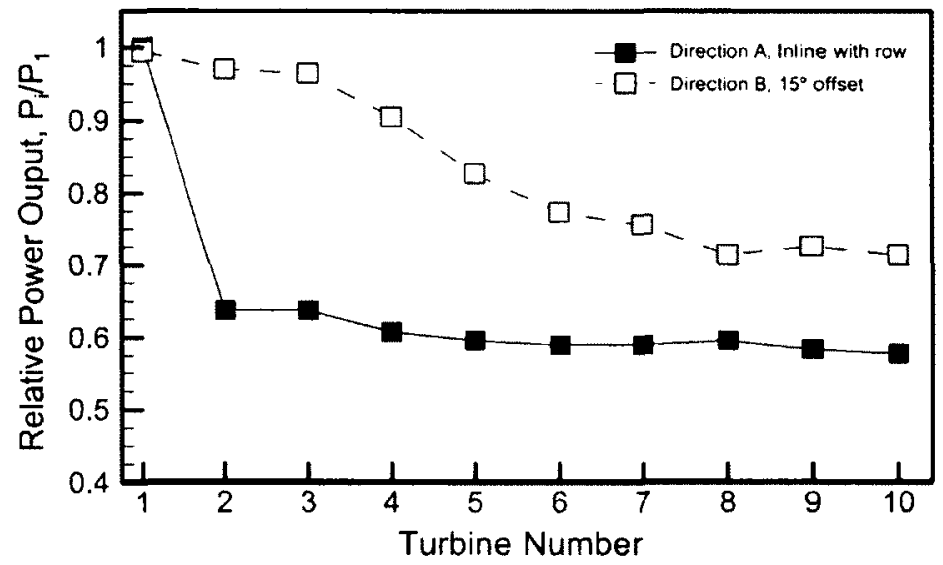

(b) Power output of one row of the Horns Rev wind farm. Adapted from [10].

Figure 1.5: Layout and power output of the offshore Horns Rev I wind farm in Denmark.

The wake effect losses in this wind farm were characterised in Ref. [10] by normalizing the power output of Turbines 1-10 in Figure 1.5(a) by the output of Turbine 1 . The normalized power output of the turbines in this particular row is plotted for two incident wind directions in Figure 1.5(b). It is clear that when the wind direction is aligned with a 
row of turbines (Direction A). a significant drop in power of approximately $35 \%$ to $40 \%$ is observed by Turbines 2-10 compared to the first turbine in the row. The overall losses in the row can be considered by calculating the array efficiency of the turbines in the row. The array efficiency can be assessed by dividing the cumulative power produced in the row by the power that would be produced by the same number of turbines in isolation (i.e. without wake effects). This results in an efficiency of $63 \%$ for this row of turbines [10]. When the wind direction is offset from the row by $15^{\circ}$ (Direction B), a more gradual loss in power from subsequent downstream turbines is observed in Figure 1.5(b). The row of turbines in the case of wind direction $B$ exhibits an efficiency of $83 \%$ [10]. In both cases, there are significant losses within the wind farm due to the exposure of downstream turbines to wind turbine wakes.

The grid-like layout that is present in the Horns Rev I wind farm and in other similar offshore wind farms differs from layouts which are typically observed for onshore wind farms. Onshore wind farms are typically built on private land and must take into consideration existing roads, transmission lines, houses, as well as topographical influences. This typically results in an irregular wind farm layout. an example of which is the Talbot wind farm located in Southern Ontario on the western shore of Lake Erie. This wind farm contains 43. $101 \mathrm{~m}$ diameter, 2.3 MW wind turbines and the schematic shown in Figure 1.6 highlights the variable separation between the wind turbines [11]. The prevailing wind directions in this region are from the west and south-west and turbines are therefore separated by at least $8.1 \mathrm{D}$ to $11.5 \mathrm{D}$ in the prevailing wind direction. Given the wind distribution at this site [12.13], turbines are more closely spaced in the lateral. or cross-wind direction. The closest hub-to-hub spacing in the lateral direction at the Talbot wind farm is 3.1D and is highlighted in Figure 1.6, but typical lateral spacing between turbine pairs is approximately $3.5 \mathrm{D}$ to $4 \mathrm{D}$. The losses due to wakes at this wind farm have been estimated using available hourly power generation data for the Talbot wind farm from the ISEO along with wind 
speed data from Environment Canada [7.12.13]. The array efficiency of the wind farm as a whole was estimated using cases where the wind farm should be operating at maximum capacity. based on observed wind speeds from Ref. [12]. It was observed that the wind farm array efficiency was often $95 \%$ to $98 \%$ when the farm should have been operating at full capacity, indicating that wake losses are approximately $2 \%$ to $5 \%$ when the wind is from the expected prevailing direction [7]. Other wind farms in the region, notably the Port Alma I wind farm and the Wolfe Island wind farm. had efficiency losses of $2 \%$ to $10 \%$ that were attributed in a similar manner to wake effects [7]. Based on the observed performance of these new, onshore wind farms, wake effects have been primarily mitigated through the staggered placement of wind turbines and large longitudinal separation distances.

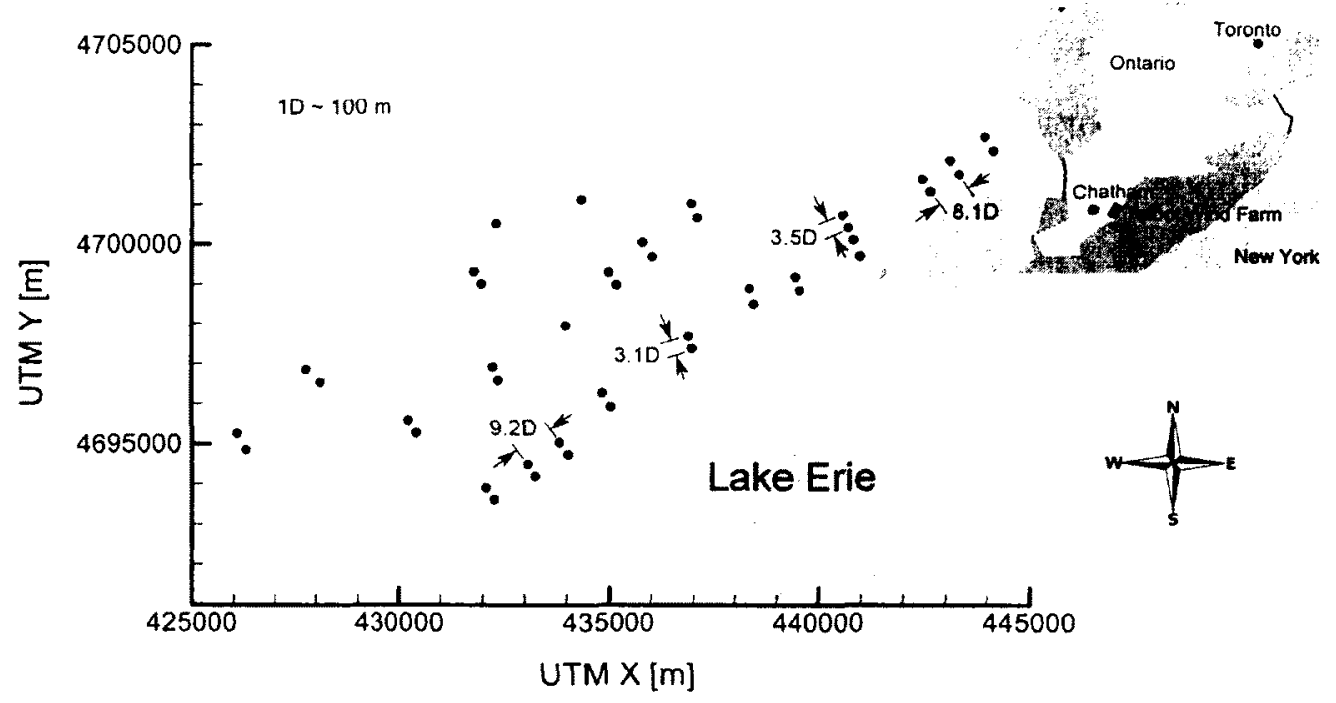

Figure 1.6: Layout of the Talbot wind farm. Data from [11].

The three above-mentioned wind farms generated more than $90 \%$ of their rated capacity only $7 \%-12 \%$ of the time, because mean annual wind speeds are considerably lower than the wind speeds considered for determining the rated capacity of the turbines [7]. A composite map of the mean annual wind speeds in Ontario at a height of $80 \mathrm{~m}$ was compiled based on the Canadian Wind Energy Atlas and is presented in Figure 1.7 [14]. Wind speeds are typically measured or modelled at a height of $80 \mathrm{~m}$ and are subsequently extrapolated up 
to the turbine's hub height. which usually ranges from $80 \mathrm{~m}-100 \mathrm{~m}$. Southern Ontario has particularly good wind resources and mean annual wind speeds approach $7-8 \mathrm{~m} / \mathrm{s}$ in this area. As previously mentioned, the mean wind speeds in Southern Ontario, the proximity to existing transmission lines, and the nearby population centres are the primary reasons why Ontario 's wind energy development has been focused in this region. The Great Lakes also hold a large amount of promise for offshore wind energy development and have mean annual wind speeds in excess of $9 \mathrm{~m} / \mathrm{s}$. While this may hold promise for the future, there is currently a provincially-mandated moratorium on offshore development in the Great Lakes.

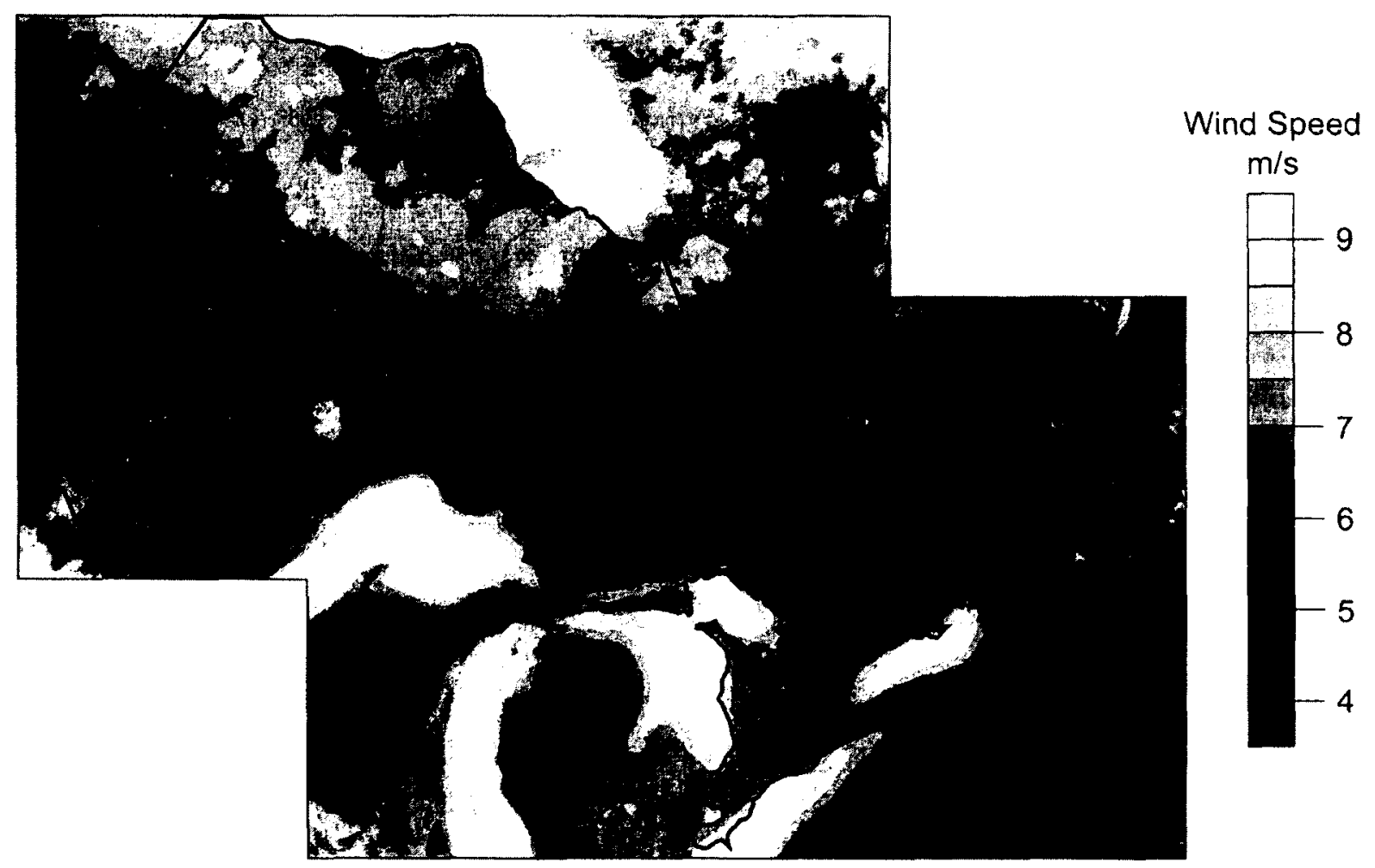

Figure 1.7: Mean wind speed distribution in Ontario at a height of $80 \mathrm{~m}$. Modified from Ref. [14].

Wind turbines have a characteristic power curve that depends on the wind speed and resembles the schematic in Figure 1.8. Wind turbines will typically only generate their maximum rated capacity at wind speeds higher than $12-14 \mathrm{~m} / \mathrm{s}$, depending on a particular turbine design. As highlighted in Figure 1.7 however, mean annual wind speeds at most 
onshore sites vary between $7-8 \mathrm{~m} / \mathrm{s}$ and mean annual wind speeds at offshore sites are closer to $9 \mathrm{~m} / \mathrm{s}$. The power curve is very sensitive to changes in wind speed below the rated wind speed and this causes turbines to generate approximately $30 \%$ of their maximum energy output on an annual basis.

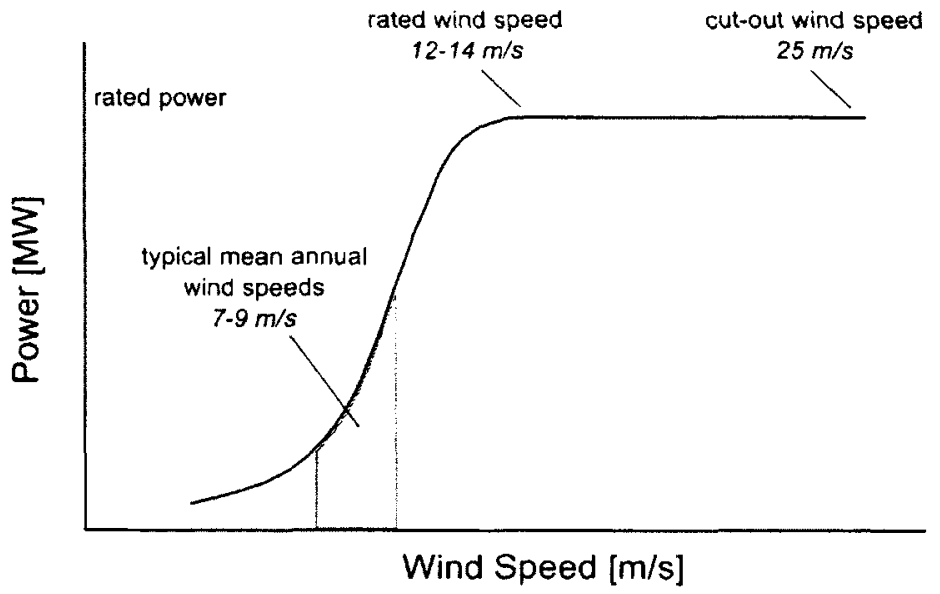

Figure 1.8: Schematic of a typical power curve.

Wind turbines will therefore generate significantly less energy than their rated capacities indicate. The annual capacity factor of a wind farm is a measure of the energy actually generated by a wind farm compared to its rated capacity. The capacity factors of 14 wind farms in Ontario were calculated from Nov 2011 to Nov 2012 using data from the IESO and are plotted in a histogram in Figure $1.9[7]$. Information regarding wind turbine shutdowns due to maintenance, repairs, ice accretion. or storms was not available and these facters were therefore not included in the analysis. Most farms exhibit a capacity factor of $30-32.5 \%$ with the highest- and lowest-performing wind farms having capacity factors of $35.9 \%$ and $26.5 \%$, respectively. The ability to improve wind farm performance at mean annual wind speeds while reducing wake effects could improve the annual capacity factor of wind farms. 


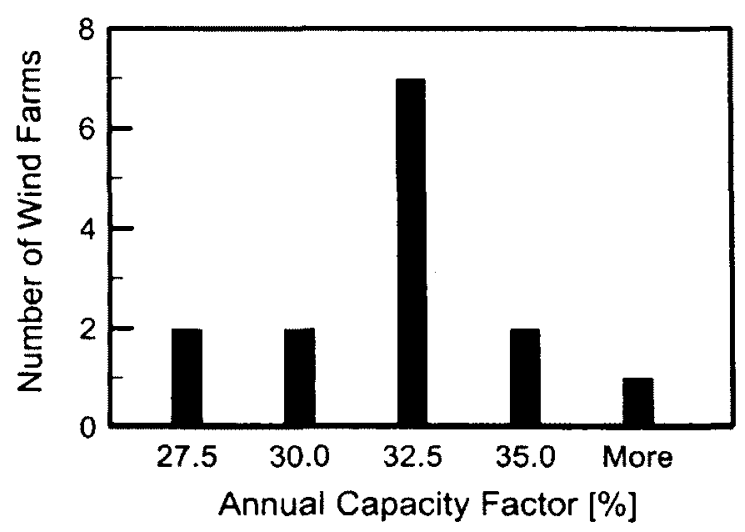

Figure 1.9: Annual capacity factors for 14 wind farms in Ontario from Nov 2011 to Nov 2012. Based on data from Ref. [7].

\subsection{Objectives}

The current thesis investigates the opportunities for increasing the power output of a wind farm by thoroughly understanding and capitalising on wake interference effects. This research may lead to new guidelines on the placement of wind turbines in a wind farm. Due to the inability of computational methods to rapidly simulate entire wind farms, the most practical method of assessing wind farm performance is through the use of scaled experiments. Significant gaps in literature were identified regarding scaling and testing limitations of such experiments and a deeper understanding of the challenges associated with small-scale wind turbine experiments had to be cultivated before investigating the issue of wind farm performance. This resulted in a thesis with the three primary objectives of (i) evaluating blockage limitations in closed test sections. (ii) improving the understanding of scaling and Reynolds number effects in small-scale testing. and (iii) establishing a method of improving the performance of wind farms by capitalising on the behaviour of wind turbine wakes.

(i) The first objective of the thesis research was to identify and establish guidelines regarding permissible amounts of blockage in scaled wind turbine experiments. The 
blockage in wind turbine experiments available in literature was observed to vary from $1 \%$ to $53 \%$. but the effect of such blockage on the development of the turbine wake had never been previously-identified. The present thesis systematically assesses the effect of increasing blockage on the tip vortex development in the wake with the goal of establishing a benchmark for testing guidelines in closed test sections.

(ii) The second objective related to the desire to develop a deeper understanding of scaling effects in low Reynolds number experiments. Commercial-scale wind turbines operate with Reynolds numbers on the order of $10^{6}$. However. because of scaling issues, these Reynolds numbers cannot be reproduced in wind tunnel experiments and it was therefore desired to assess the ability of scaled models to operate in a low Reynolds number testing environment. The present thesis also seeks to identify the flow regime where properties such as the wake expansion and force coefficients can be credibly related to full-scale wind farms.

(iii) The third objective of the work was to develop and evaluate a methodology that could be used to increase the power output of a wind farm. It was desired to investigate wind farm configurations that would not simply mitigate wake effects, but could also capitalize on the behaviour of the incident wind and the wake as a means to improve wind farm performance.

\subsection{Approach}

There are two possible methods of resolving wind turbine performance and wake development: with experiments or computational simulations. The merits of the two methods will be discussed in this Section and the selected approach will be highlighted.

Wind turbine experimental data can be obtained from scaled-models in a wind tunnel or 
from full-scale turbines in the field. Wind tunnel experiments can offer turbulence and speed information at several downstream planes from a rotor in a controlled environment. Scaling effects must be considered in scaled wind turbine experiments and this generally violates Reynolds number similarity. Also, the wind tunnel test section must be long enough to produce a representative atmospheric boundary layer, which restricts the size of the scaled rotor to be used. Typically, hot-wire anemometry (HWA) or particle image velocimetry (PIV) systems are used to characterise the wake and direct force and voltage measurements are used to characterise rotor forces and power [15-17]. Hot-wire measurements can generate highly-resolved speed data, but are typically restricted to point measurements and necessitate a traversing apparatus [16]. While PIV permits the spatial variation in the wake to be characterised, a challenging environment for PIV is encountered when studying the wake behind a large wind turbine experiment [17]. Despite these limitations, wind tunnel experiments have led to the development of currently-used wake models and have provided a large amount of physical insight into wake behaviour. In addition to wind tunnel experiments, several wind turbine field experiments have been performed which often focus on determining wake deficit profiles and power losses in wind farms $[18,19]$. Arrays of meteorological masts instrumented with anemometers and wind vanes have traditionally been used to conduct field experiments. Even with several arrays of masts, there is an inherent difficulty in accurately measuring the entire flowfield. Recently, sonic (SODAR) and laser-based (LIDAR) flow measurement systems have been used to characterise inflow and wakes and may produce very detailed full-scale measurements in the future [19].

On the other hand. computational simulations can offer the ability to study the wake in greater detail in more isolated conditions than experiments. However, currently-used wake models and grid-based computational fluid dynamics (CFD) codes suffer from many shortcomings. Firstly. wake models are typically only accurate in situations for which they have been calibrated as they rely on empirical constants. Secondly. conventional grid-based 
Reynolds Averaged Navier-Stokes (RANS) CFD codes can accurately capture the blade loading and power generation. but the numerical dissipation that is inherently present in grid-based codes causes the wake to artificially dissipate much faster (within 2-3 rotor diameters) than in reality. This may be somewhat mitigated with very fine grids in the wake, but this imposes a high computational cost which presently prohibits the completion of multi-rotor simulations. In fact, very few multi-turbine simulations have been presented in literature due to these challenges. One class of CFD methods, Vortex Particle Methods, however, is particularly well suited to rotary-wing simulations and can predict a wake structure very accurately, virtually free of numerical dissipation. The grid-free nature of vortex particle codes also eliminates the complex meshing requirements of grid-based CFD. This is the method used at Carleton University, and it has been used to simulate the interference of two turbines in 2008 [20].

Compared to the challenges associated with CFD solvers at the wind farm level, an experimental approach had many benefits. including the ability to quickly assess various wind turbine configurations and the ability to develop data sets for future validation. The objectives of this thesis were therefore addressed using a predominantly experimental approach. The range of techniques that were implemented include quantitative flow visualisation, direct force measurements, power measurements with scaled wind turbines, and hot-wire speed measurements. These experimental methods were supported and complemented by a range of computational tools. including blade element momentum theory and discrete vortex method computational simulations.

\subsection{Structure of the Thesis}

The thesis is divided into seven chapters. Background material and a survey of pertinent literature is presented in Chapter 2. The experimental methods that were applied in the 
water channel and wind tunnel are described in Chapter 3. along with the method used for the discrete vortex method computational simulations. The results chapters are presented in the order in which the research was conducted. Chapter 4 presents the results that were established during the blockage campaign conducted in Carleton's water channel. The improved understanding of scaling and Reynolds number effects is described in Chapter 5 . The results of the wind farm performance measurement campaigns are presented in Chapter 6 along with the wake speed profiles determined using hot-wire anemometry. Chapter 7 subsequently identifies the major conclusions of the research. summarises the academic contributions made throughout the thesis work. and highlights recommendations for future work. 


\section{Chapter 2}

\section{Background and Literature Review}

The effects of blockage, scaling effects. and the analysis of wind farm configurations were all considered using a predominantly experimental approach supported by a small number of computational simulations. This Chapter will describe the relevant background material relating to wind turbine aerodynamics, including blade aerodynamics, a description of the atmospheric boundary layer (ABL), and near and far wake characteristics. A survey of existing wind turbine and wind farm experiments follows the aerodynamic background material. Finally, a summary of the various means of studying wind turbine performance with computational methods will be presented.

\subsection{Wind Turbine Aerodynamics}

The aerodynamic environment of wind turbines involves a number of unsteady threedimensional effects. Their operational environment includes the effects of atmospheric turbulence, the atmospheric boundary layer, directional and temporal variations in wind shear and thermal stratifications [8]. These unsteady effects cause a wide range of unsteady 
loads. which are compounded by the effects of turbine yaw misalignment, tower and hub interference, and turbine wake interaction in wind farms.

\subsubsection{Blade Aerodynamics}

Horizontal axis wind turbines generate torque as a result of the lift produced by the rotor blades. A schematic of the aerodynamic loads generated by a turbine rotor can be seen in Figure 2.1. The airfoil cross-section inset in Figure 2.1 illustrates that the wind turbine blades have a positive pitch angle $(\beta)$ into the wind direction. This results in a low angle of attack $(\alpha)$ and a lift vector that is inclined in the direction of rotation. The lift and drag vectors can be decomposed to produce a tangential force $\left(F_{T a n}\right)$ in the direction of rotation and a thrust force $\left(F_{\text {Thrust }}\right)$ in the axial direction. The tangential and thrust forces of a wind turbine determine its performance as they relate to the torque generation $(Q)$ of the rotor and the velocity deficit in the wake. respectively.

The thrust force, torque, and power of a wind turbine are frequently non-dimensionalised in order to compare various rotor designs. The thrust $\left(C_{T}\right)$. torque $\left(C_{Q}\right)$. and power coefficients $\left(C_{P}\right)$ are defined in Equations (2.1) to (2.3) and are functions of the air density $(\rho)$, the swept area of the rotor blades $\left(A_{\text {swept }}\right)$, and the freestream wind speed $\left(U_{\infty}\right)$. The thrust coefficient relates to the momentum deficit in the wake and the power coefficient is frequently interpreted as the efficiency of a rotor. as it represents the relationship between the power extracted by the rotor and the power available in the area swept by the rotor blades. The mechanical power generated by the turbine $(P)$ is calculated as $P=Q \omega$. where $\omega$ is the rotational speed of the turbine.

$$
C_{T}=\frac{F_{\text {Thrust }}}{0.5 \rho A_{\text {swept }} U_{\infty}^{2}}
$$




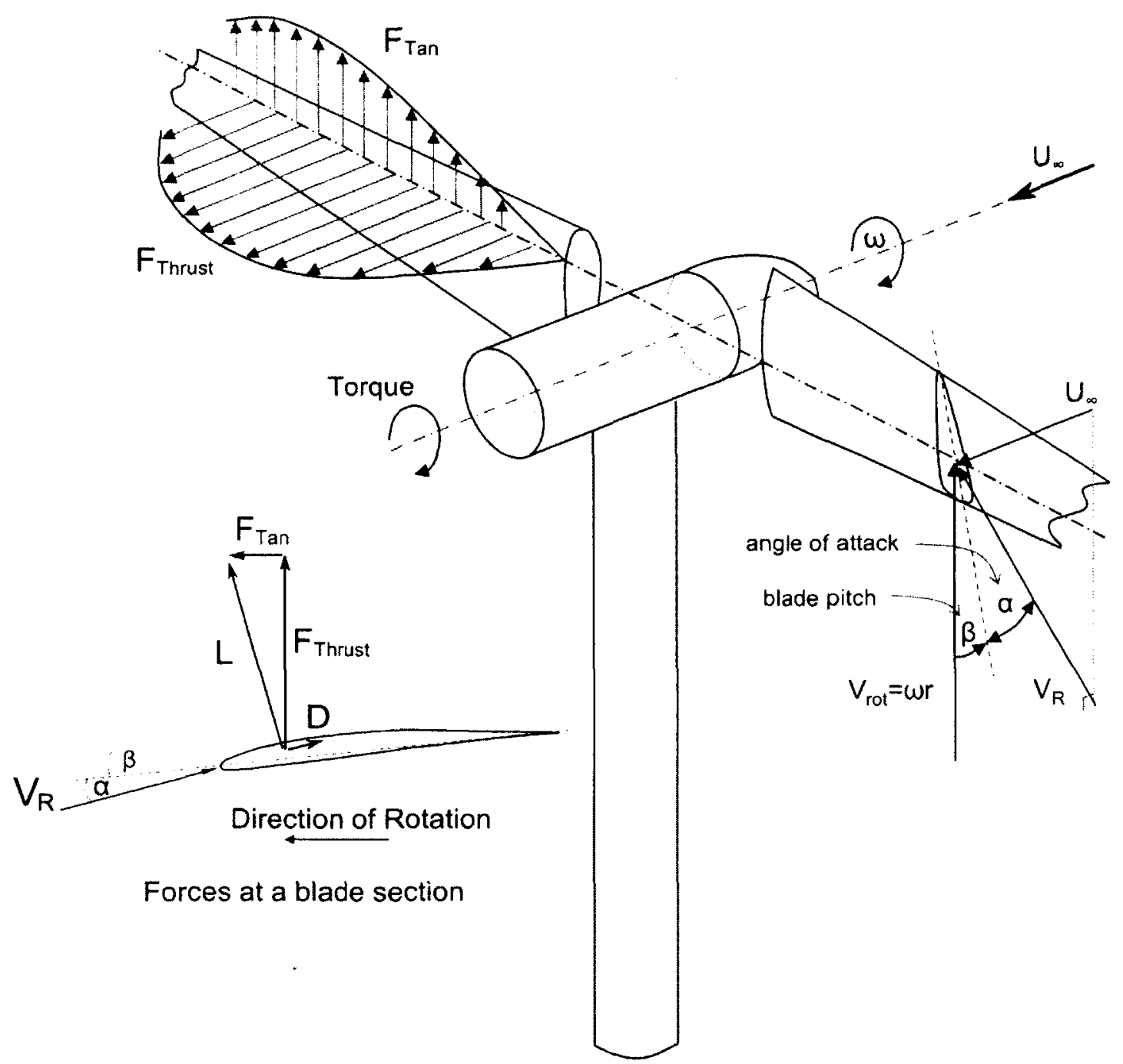

Figure 2.1: Loads generated by a wind turbine rotor.

$$
\begin{gathered}
C_{Q}=\frac{Q}{0.5 \rho R A_{\text {swept }} U_{\infty}^{2}} \\
C_{P}=\frac{P}{0.5 \rho A_{\text {swept }} U_{\infty}^{3}}
\end{gathered}
$$

The tip speed ratio of a turbine represents the ratio of the velocity of the blade tip to the incident wind speed and is defined in Equation (2.4), where $\omega$ is the rotational speed 
of the turbine. $R$ is the rotor radius. and $U_{x}$ is the inflow speed. The tip speed ratio of commercial-scale wind turbines typically ranges from $6-8$ over the range of mean annual wind speeds.

$$
\lambda=\frac{\omega R}{U_{x}}
$$

The lift and drag properties of a wind turbine will be associated to the Reynolds number of a local blade section. In order to compare different turbine designs, the Reynolds number based on the blade speed at the tip $\left(U_{t i p}\right)$ and the chord at the tip $\left(c_{t i p}\right)$ is defined in Equation (2.5), where $\nu$ is the kinematic viscosity of the fluid. An alternate definition of the Reynolds number used to compare wind turbine designs is based on the turbine diameter and the freestream wind speed.

$$
R e_{t i p}=\frac{U_{t i p} c_{t i p}}{\nu}
$$

The power generated by horizontal axis wind turbines can either be regulated using pitch-controlled or stall-controlled turbine blades. In pitch-controlled turbines, the pitch angle of the turbine blades is adjusted based on the incident wind speed measurements during operation to maintain constant rotor rpm and to regulate power output. This allows the angle of attack to remain low throughout the operational regime of the turbine and allows the rated turbine power to be controlled over the rated wind speeds. If the incident speed is too high and risks damaging a turbine. the blades are rotated to the feathered position and the turbine is stopped [9].

Stall-controlled turbines however. have a fixed blade pitch angle and their blades are designed to operate in a fully stalled flow regime at and above the turbine's rated wind speed. Stall-regulated blades rely on passive stall control to regulate the power generated 
by the rotor and to maintain constant rotational frequency. This approach is described schematically in Figure 2.2 where a turbine blade section is shown at both a low wind speed and a high wind speed. The fixed blade pitch angle $\left(\beta_{1}\right)$ is assumed to be positive towards the feathered position and the tangential velocity $\left(V_{\text {rot }}\right)$ of a particular spanwise section is assumed to be constant throughout the operational range of the turbine. Figure 2.2 demonstrates that the blade section angle of attack $(\alpha)$ will increase with the wind speed. At wind speeds that correspond to the rated power of a turbine, $\alpha$ will be higher than the critical angle of attack $\left(\alpha_{\text {stall }}\right)$ where the blade section begins to experience stalled flow. At the rated turbine power, a varying degree of stall occurs over the blade sections and the rotor power is controlled by the amount of aerodynamic stall occurring over the span of the rotor blade. There has recently been renewed interest in stall-regulated rotors for offshore wind turbines, since eliminating pitch-control mechanisms could reduce complexity, weight. and cost.

\subsubsection{The Atmospheric Boundary Layer}

Wind turbines operate within the lower portion of Earth s atmosphere and are subjected to wind shear from the $\mathrm{ABL}$ that creates unsteady rotor loading. The $\mathrm{ABL}$ height can range from approximately $200 \mathrm{~m}$ to over $1 \mathrm{~km}$ depending on the thermal stratification in the atmosphere [21]. Unstable atmospheric conditions typically occur during the day when surface heating creates large-scale vertical convection cells and a thick ABL [21]. Stable atmospheric conditions will typically occur at night when the surface is cooler than the surrounding air. resulting in a relatively thin $\mathrm{ABL}$. In this case. turbulence is not typically generated by large-scale eddies but is instead dominated by frictional effects and the surface roughness of the ground [21]. Neutral atmospheric conditions will occur in the late afternoon in strong winds if the air rising from the heated ground is in thermal equilibrium with the ambient air. In addition to buoyancy effects. surface roughnes's will modify the shape and 


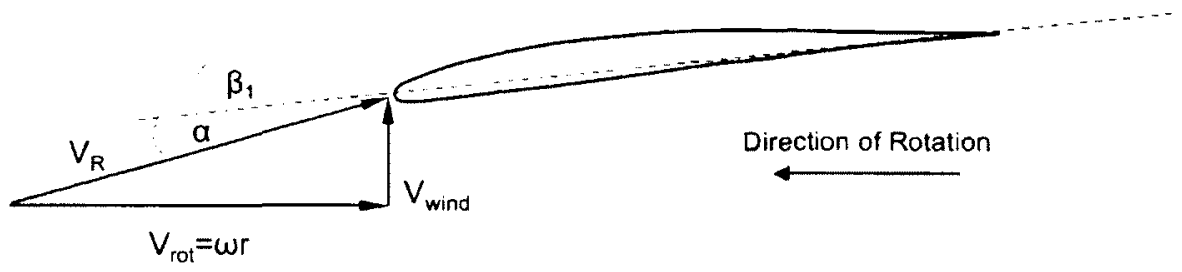

(a) Low wind speed.

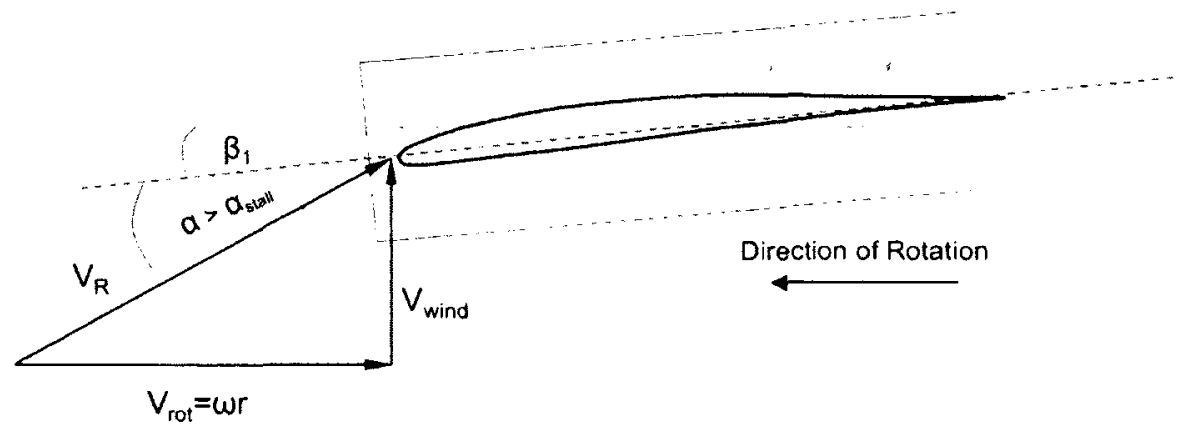

(b) High wind speed - stall control.

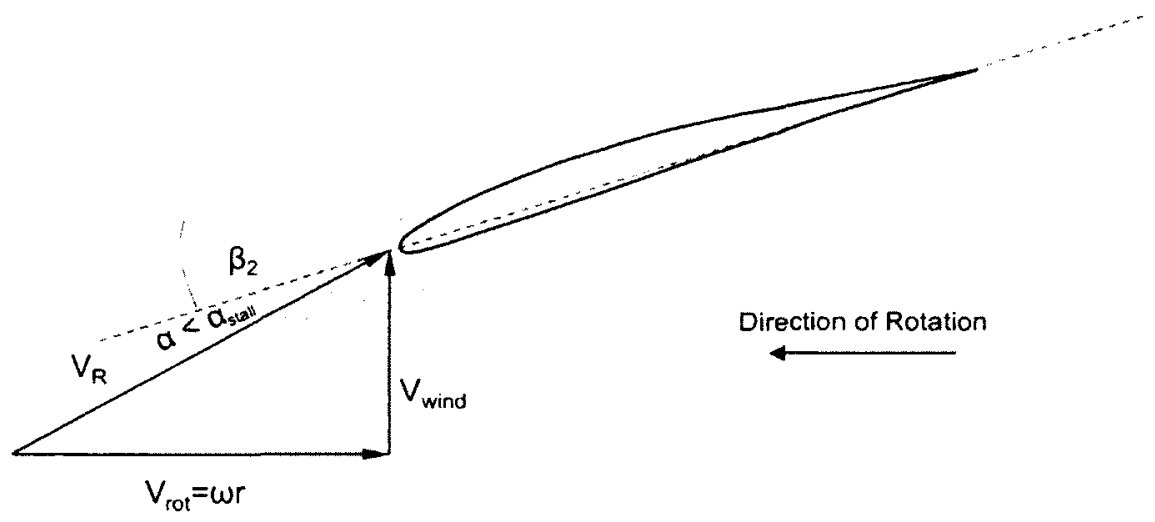

(c) High wind speed - pitch control.

Figure 2.2: Regulating power output using stall control or pitch control.

thickness of the atmospheric boundary layer. A schematic of the atmospheric boundary layer profiles over smooth offshore and rough onshore terrain is presented in Figure 2.3. This highlights the gradient in the boundary layer that onshore wind turbines. which typically have a hub height of $100 \mathrm{~m}$, are exposed to.

The profile of the atmospheric boundary layer is frequently approximated using a power law, as described by Equation (2.6), where $U$ is the speed at a particular height $z . U_{\delta}$ is 


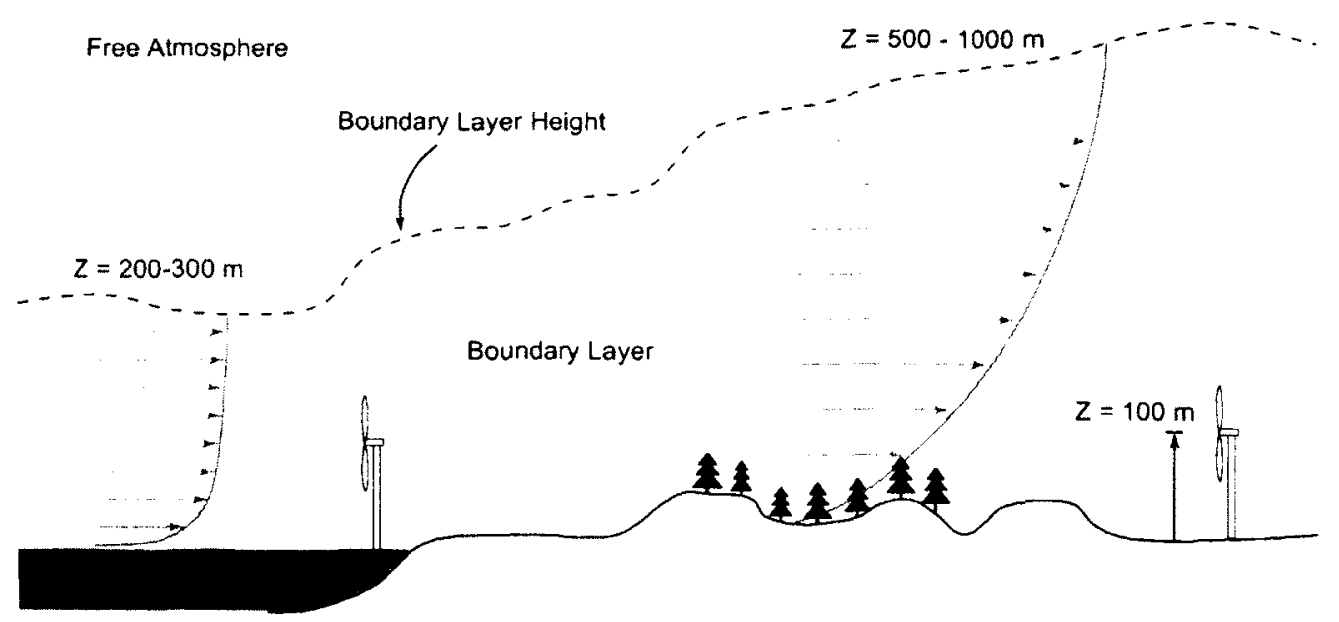

Figure 2.3: Schematic of the atmospheric boundary layer over smooth and rough terrain. Not to scale.

the speed at a reference height $\delta$, and $\alpha$ is the desired power law exponent. The power law exponent is related to a roughness length that characterises the flow over a particular type of terrain. Although the ABL profile is typically approximated with a power law in wind energy research, the profile is more commonly approximated with a logarithmic profile in the atmospheric sciences.

$$
\frac{U}{U_{\delta}}=\left(\frac{z}{\delta}\right)^{a}
$$

\subsubsection{Wake Development}

The evolution of the wake will depend on the atmospheric turbulence intensity. the surface roughness of the local environment, the presence of wind shear and gusts, and any topographic effects. The wake is typically characterised by two regions: the near-wake and the far wake, as indicated in Figure 2.4(a). The near-wake region typically extends 2 diameters (D) to $4 \mathrm{D}$ downstream of the rotor and the far wake can extend to $15-20 \mathrm{D}$ downstream, depending on the atmospheric conditions [9]. 
In uniform inflow conditions, the initial velocity distribution in the near-wake has a relatively flat profile. as seen in Figure 2.4(a) and is dependent on the ambient wind speed and the rotor thrust. Immediately downstream of the rotor. the velocity difference between the freestream and the wake creates a shear layer that will thicken downstream of the rotor because of turbulent mixing [21]. The wake expands as momentum is transferred to the wake through the turbulent mixing between the wake and the freestream allowing the velocity deficit to gradually recover downstream [22]. The mixing process and the shear layer thickening in the near-wake is illustrated schematically in Figure 2.4(a) and the centreline speed upstream and downstream of the rotor in Figure 2.4(b) illustrates the deceleration upstream of the rotor and the gradual velocity deficit recovery.

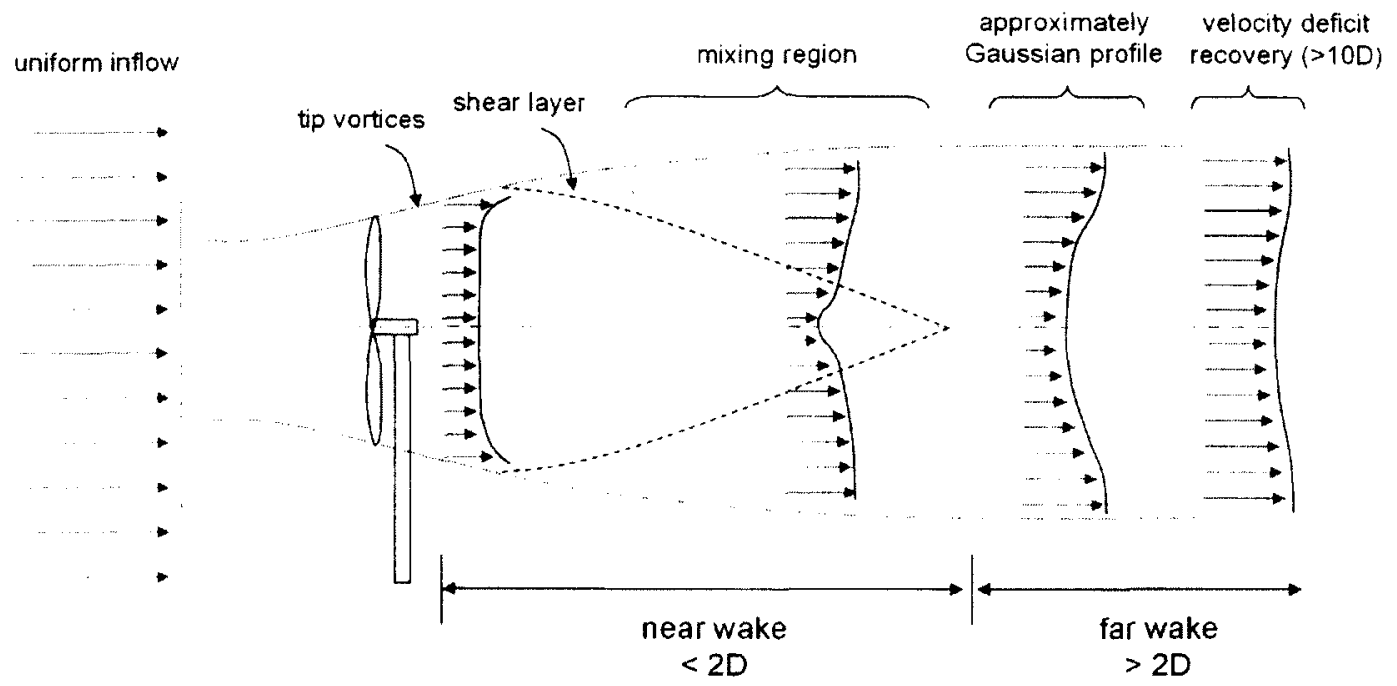

(a) Schematic of the near and far wake in uniform flow. Adapted from [21].

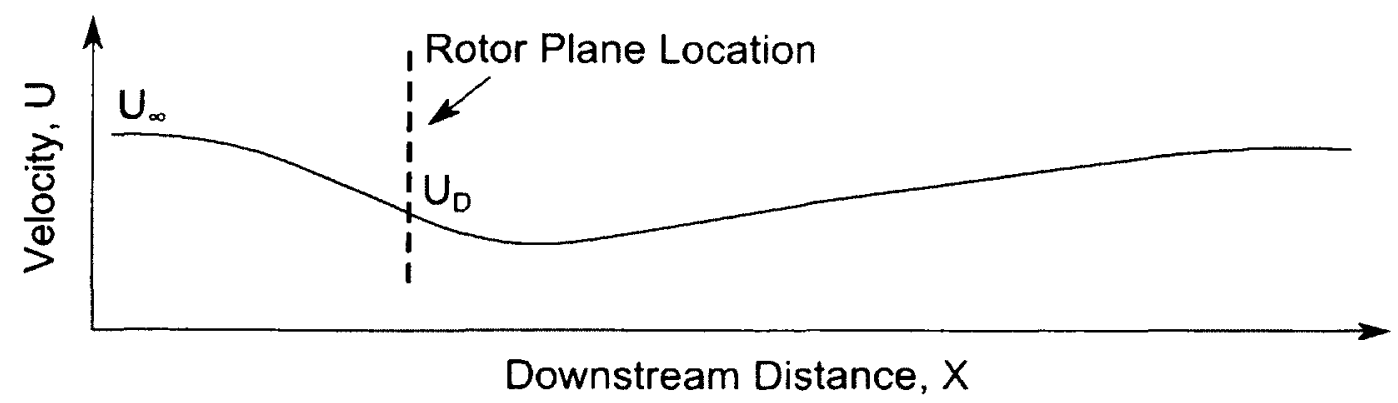

(b) Centreline flow speed upstream and downstream of a turbine.

Figure 2.4: Description of wind turbine wake evolution. 
The momentum extraction at the rotor plane will slow down the upstream air approaching the rotor. The axial induction factor $(a)$ is used to characterise the speed of the incident flow at the rotor plane $\left(U_{D}\right)$ according to Equation (2.7). The axial induction is related to the thrust coefficient of a turbine and the downstream wake expansion profile and characterises the decrease in mean speed upstream through the rotor plane.

$$
U_{D}=U_{\infty}(1-a)
$$

A larger thrust coefficient produced by the rotor will create a higher axial induction factor and will result in a larger velocity deficit behind the rotor. This will in turn create a larger shear between the freestream and the near wake. The end of the near wake corresponds to the location where the shear layer has thickened to the turbine axis, as indicated in Figure 2.4(a). In addition to the atmospheric turbulence and the mixing occurring in the shear layer, the shed vortices from the blades and mechanical turbulence from the nacelle and turbine tower will also increase the turbulence in the wake.

The idealised model presented earlier in Figure 2.4 neglects important flow features such as tip vortices and the $\mathrm{ABL}$ that alter the representation of the wake. The lift-generating nature of wind turbine blades results in vortices which are continually shed into the wake of the turbine. The vorticity along the blades will roll-up into a tip vortex and into a weaker root vortex and these helical structures propagate downstream. A schematic of the tip vortex shed from a single blade is shown in Figure 2.5(a). The cut-plane through the helical tip vortices shown in Figure 2.5 (b) highlights the tip vortices shed by successive blades and is based on an image recorded recorded during the current research. The tip vortices will merge. diffuse. and grow in diameter in the near wake region. and although the distinct tip vortex cores are only present for 1-3D downstream of a turbine, the presence of the merged vortex structures can be sensed further downstream in the far wake. 


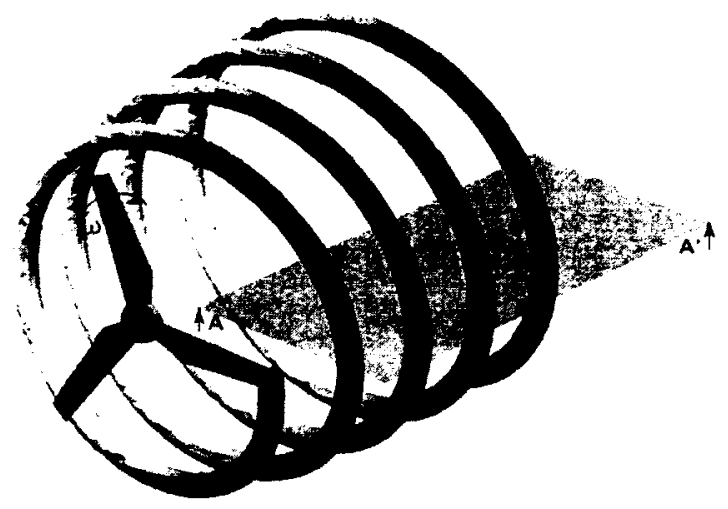

(a) Schematic of a helical tip vortex.

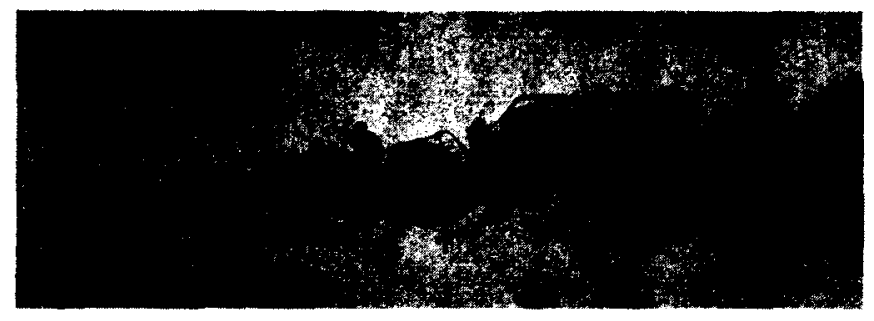

(b) Tip vortices at plane A-A'.

Figure 2.5: Representation of tip vortex shedding from a three-bladed wind turbine.

In addition to the presence of helical tip vortices, the presence of the ground and the velocity profile of the $A B L$ will cause the velocity and turbulence intensity profiles in the wake to differ from the idealised wake behaviour previously-highlighted in Figure 2.4(a). There will be a larger amount of mixing in the upper part of the wake and less mixing in the lower part of the wake due to the shear created by the ABL. This results in an asymmetric velocity profile and two peaks in the turbulence intensity $(T u)$ profile in the wake of the rotor, as highlighted by the schematics in Figure 2.6. The turbulence intensity is defined in Equation (2.8) and is normalised using the mean reference speed at hub height for consistency with results presented in wind turbine literature [16].

$$
T u=\frac{U_{r m s}}{U_{r e f}^{r}}
$$


Figure 2.6(a) illustrates the gradient in the ABL upstream of the turbine, and normalised velocity profiles in the wake that are representative of conditions observed 3D. 5D. 7D. and $9 \mathrm{D}$ downstream of a turbine. Likewise. the turbulence intensity profile in the upstream $\mathrm{ABL}$ and the turbulence intensity profiles in the wake 5D, 7D. and $9 \mathrm{D}$ downstream of a turbine are highlighted in Figure 2.6(b). Note that the turbine hub height is assumed to be located at $Z / D=1$ and the turbine blades would extend vertically from $Z / D=0.5$ to $Z / D=1.5$. In the far wake, the velocity deficit recovers more rapidly than the added turbulence in the wake decays. The velocity deficit generally recovers in approximately 10D to $15 \mathrm{D}$ downstream but the increased turbulence intensity in the wake can be observed $15 \mathrm{D}$ to $20 \mathrm{D}$ downstream of a rotor and this behaviour increases the unsteady loading on downstream turbines in a wind farm $[9.16]$.

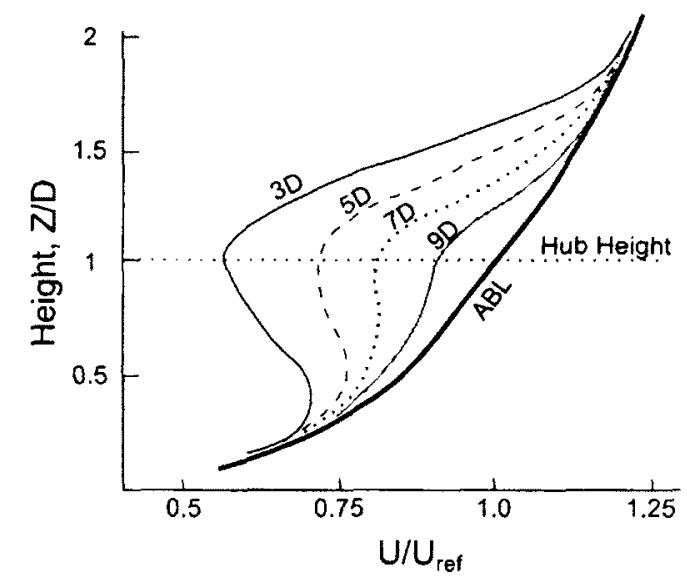

(a) Velocity profile in the wake.

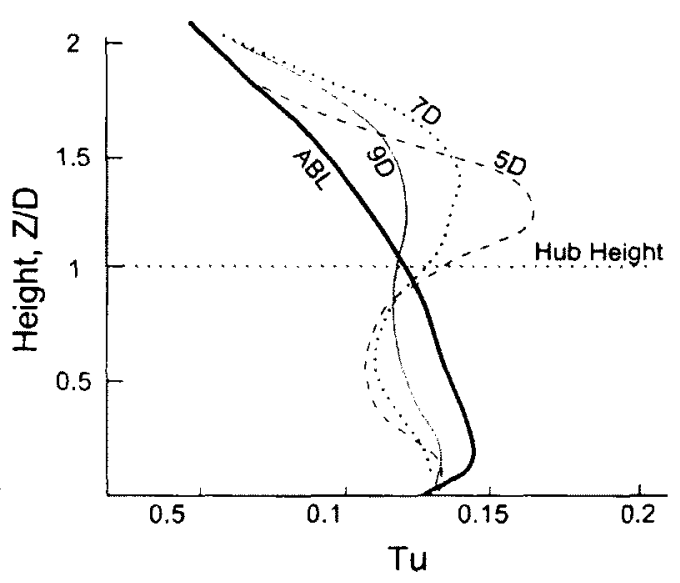

(b) Turbulence intensity profile in the wake.

Figure 2.6: Schematic of velocity and turbulence intensity profiles in an atmospheric boundary layer. Adapted from [16].

\subsubsection{Wind Farm Performance}

The power output of a wind farm is typically degraded due to the wake effects present in grid-aligned configurations. The overall efficiency of a wind farm including wake effects can be characterised using the array efficiency $\left(\eta_{\text {array }}\right)$ parameter. The array efficiency of a 
wind farm can be calculated with Equation (2.9). where $N$ is the number of turbines being considered. $P_{i}$ is the power output of a particular turbine. and $P_{o}$ is the power output of a clean. undisturbed turbine, where $P_{o}$ is typically selected as the power output from an upstream turbine that is not exposed to any wake effects. In this way, the array efficiency can be interpreted as the power output generated by the wind farm considering wake effects compared to the same wind farm without wake effects.

$$
\eta_{\text {array }}=\frac{1}{N} \sum_{i=1}^{N} \frac{P_{i}}{P_{o}}
$$

\subsection{Review of Existing Experiments}

This section describes many of the recent experimental efforts that have attempted to characterise various aspects of horizontal axis wind turbine performance. The review will highlight undertakings that considered isolated rotor performance, blockage effects. challenges associated with scaling, wake interaction effects. examples of scaled wind farms. and will identify some of the novel approaches that have been taken to increase the performance of wind farms. A detailed review of wind turbine experiments that focused on the near wake and far wake regions was given by Vermeer et al. in 2003 and summarized the major experimental efforts conducted between 1978 and 2001 [19]. A summary of the pertinent experiments to this thesis that have been published (with a few exceptions) since 2001 has been compiled in Table 2.1. The primary dimensions of the rotors and the test sections in which they were evaluated. the blockage ratio, Reynolds numbers, and information regarding the available measurements in each campaign are summarized in Table 2.1. Only the name of the first author associated with each experiment is shown in Table 2.1 for compactness. 
Table 2.1: A summary of recently-published wind turbine experiments.

\begin{tabular}{|c|c|c|c|c|c|c|c|c|c|c|}
\hline Author & Diameter & $\begin{array}{l}\text { Tunnel } \\
\text { Dimensions } \\
{[\mathbf{m}]}\end{array}$ & $\begin{array}{l}\text { Test } \\
\text { Section }\end{array}$ & $\begin{array}{l}\text { Blockage } \\
{[\%]}\end{array}$ & $R e_{c}$ & $\operatorname{Ren}$ & Airfoil & Blades & Facility & Measurements \\
\hline $\begin{array}{l}\text { Adaramrola } \\
{[23]}\end{array}$ & 0.9 & $2 \times 2.7$ & closed & 11.8 & 120,000 & 685,000 & unknown & 3 & $\begin{array}{l}\text { Norwegian Uni- } \\
\text { versity of Sci- } \\
\text { ence and Tech- } \\
\text { nology }\end{array}$ & Forces. $C_{P}$ \\
\hline Ainstie $[24\}$ & 0.27 & $9.1 \times 2.7$ & closed & 0.2 & $\begin{array}{l}11,500- \\
67,600\end{array}$ & 95,000 & $\begin{array}{l}\text { custom, } \\
6.8 \% \\
\text { camber }\end{array}$ & 3 & $\begin{array}{l}\text { CEGB March- } \\
\text { wood Engineer- } \\
\text { ing laboratory }\end{array}$ & HWA, $C_{r}$ \\
\hline Akay [25] & 2 & $3($ diam $)$ & open & 44.4 & 320,000 & $1,400,000$ & $\begin{array}{l}\text { DU-96-W- } \\
180\end{array}$ & 2 & $\begin{array}{l}\text { Open Jet Facil- } \\
\text { ity, TU Delft }\end{array}$ & PIV near wake \\
\hline Al-A badi $[26\}$ & 0.4 & $1.4 \times 1.87$ & closed & 4.8 & $\begin{array}{l}55,500- \\
82,800\end{array}$ & $\begin{array}{l}318,000 \\
480,000\end{array}$ & Clark Y & 3 & $\begin{array}{l}\text { Friedrich- } \\
\text { Alexander } \\
\text { University }\end{array}$ & $c_{r}$ \\
\hline $\begin{array}{l}\text { Alfredsson } \\
|27|\end{array}$ & 0.25 & $2.1 \times 1.5$ & closed & 1.8 & $\begin{array}{l}38,000- \\
65,000\end{array}$ & $\begin{array}{l}94,000- \\
134,000\end{array}$ & $\begin{array}{l}\text { cambered } \\
\text { plate }\end{array}$ & 2 & $\mathrm{KTH} A B L$ & $\begin{array}{l}\text { HWA, } C_{P} \text {, smoke } \\
\text { viz }\end{array}$ \\
\hline Aubrun $[28\}$ & $0.1-0.3$ & $2 \times 2$ & closed & $0.2-1.8$ & - & $\begin{array}{l}66,000- \\
198,000\end{array}$ & - & $\begin{array}{l}\text { Porous } \\
\text { disc }\end{array}$ & $\begin{array}{l}\text { Université } \\
\text { d'Orléans ABL }\end{array}$ & $\mathrm{HWA}, \mathrm{C}_{\mathrm{T}}$ \\
\hline Aubrun [29] & 0.416 & $2 \times 2$ & closed & 3.4 & 14,000 & 69,000 & unknown & 3 & $\begin{array}{l}\text { Université } \\
\text { d'Orléans ABL }\end{array}$ & HWA, $C_{T}$ \\
\hline Bahaj $[30]$ & 0.8 & $2.4 \times 1.2$ & closed & 17.4 & 170,000 & $1.350,000$ & $\begin{array}{l}\text { NACA } 63- \\
8 x x\end{array}$ & 3 & $\begin{array}{l}\text { Tow-tank } \\
\text { Southampton } \\
\text { Institute }\end{array}$ & forces \\
\hline Bahaj $[30]$ & 0.8 & $1.8 \times 3.7$ & closed & 7.5 & 170.000 & $1,350,000$ & $\begin{array}{l}\text { NACA } 63- \\
8 \times x\end{array}$ & 3 & $\begin{array}{l}\text { Cavitation } \\
\text { Tunnel, } \\
\text { QinetiQ } \\
\end{array}$ & forces \\
\hline Barber $[31\}$ & 0.3 & $1 \times 1$ & closed & 7.1 & 134,000 & 900,000 & 5809 & 3 & ETH-Zurich & ADV wake velocity \\
\hline $\begin{array}{l}\text { Burdett } \$ 32 . \\
33\}\end{array}$ & $0.3-0.5$ & $0.61 \times 0.61$ & closed & $19-53$ & $\begin{array}{l}55,000- \\
300,000 \\
\end{array}$ & $\begin{array}{l}50,000- \\
210,000 \\
\end{array}$ & S823 & 3 & $\begin{array}{l}\text { Baylor Univer- } \\
\text { sity }\end{array}$ & $c_{P}$ \\
\hline $\begin{array}{l}\text { Chamorro } \\
{[16,34]}\end{array}$ & 1) 1524 & $1.7 \times 1.7$ & closed & 0.6 & 6.120 & 25,000 & $\begin{array}{l}\text { GWS EP } \\
6030\end{array}$ & 3 & $\begin{array}{l}\text { University of } \\
\text { Minnesota }\end{array}$ & HWA wake \\
\hline
\end{tabular}

Continued on next page 
Table 2.1 - continued from previous page

\begin{tabular}{|c|c|c|c|c|c|c|c|c|c|c|}
\hline Author & Diameter & $\begin{array}{l}\text { Tunnel } \\
\text { Dimensions } \\
\text { [m] }\end{array}$ & $\begin{array}{l}\text { Test } \\
\text { Section }\end{array}$ & $\begin{array}{l}\text { Blockage } \\
{[\%]}\end{array}$ & $R e_{c}$ & Ren & Airfoil & Blades & Facility & Measurements \\
\hline Chen [35] & $0.47-0.78$ & $1.3 \times 1.3$ & closed & $10.2-28.3$ & $\begin{array}{l}23,000- \\
55,600\end{array}$ & $\begin{array}{l}310,000- \\
620,000\end{array}$ & $\begin{array}{l}\mathrm{NACA} \\
\mathbf{4 4 1 5}\end{array}$ & 6. 12 & $\begin{array}{l}\text { Tamkang Uni- } \\
\text { versity }\end{array}$ & $\begin{array}{l}\text { forces, upstream ve- } \\
\text { locity (Pitot) }\end{array}$ \\
\hline Cho $[36 \mid$ & 2 & $5 \times 3.75$ & open & 16.7 & $\begin{array}{l}134,000 \\
253.000\end{array}$ & $\begin{array}{l}6.6 \mathrm{E} 6- \\
12 \mathrm{E} 6\end{array}$ & see $[36]$ & 3 & $\begin{array}{l}\text { Korean } \\
\text { Aerospace } \\
\text { Research } \\
\text { Institute }\end{array}$ & Forces. $C_{\Gamma}$ \\
\hline Dan-mei [37] & 0.5 & 0.9 (diam) & closed & 30.9 & unknown & $O\left(10^{5}\right)$ & $\begin{array}{l}\text { NREL } \\
\text { S } 809\end{array}$ & 3 & $\begin{array}{l}\text { Shanghai } \\
\text { Jiao Tong } \\
\text { University }\end{array}$ & forces. HWA wake \\
\hline Grant [38] & 0.9 & $1.24($ diam,oct $)$ & open & 50.0 & 105,000 & 370,000 & $\begin{array}{l}\text { NACA } \\
4611\end{array}$ & 2,3 & $\begin{array}{l}\text { Heriot-Watt } \\
\text { University }\end{array}$ & PIV \\
\hline Grant [39] & 1 & $2.13 \times 1.61$ & closed & 22.9 & 200.000 & 500,000 & $\begin{array}{l}\mathrm{NACA} \\
4415\end{array}$ & 2 & $\begin{array}{l}\text { Univ of Glas- } \\
\text { gow }\end{array}$ & blade forces, PIV \\
\hline Haans $|40|$ & 12 & $2.24(\mathrm{diam})$ & open & 28.7 & $\begin{array}{l}175.0000- \\
290.000\end{array}$ & $1,150,000$ & $\begin{array}{l}\mathrm{NACA} \\
0012\end{array}$ & 2 & TU Delft & smoke viz. \\
\hline Hossain $|41|$ & 0.05 & $3 \times 1.8$ & closed & 0.04 & unknown & 30.000 & unknown & 4 & Fujita Corp & HWA. PIV \\
\hline $\begin{array}{l}\text { Maeda } \\
\{42,43]\end{array}$ & 0.5 & $3.6($ diam $)$ & open & 2 & 113.000 & 232.000 & unknown & 2 & Mie University & Pitot tubes. HWA \\
\hline Massouh $[44]$ & 0.5 & $1.35 \times 1.65$ & closed & 8.8 & 78.000 & 308,000 & unknown & 3 & $\begin{array}{l}\text { Ecole Nationale } \\
\text { Supérieure } \\
\text { d'Arts et } \\
\text { Métiers }\end{array}$ & PIV \\
\hline Medici $[45]$ & 0.18 & $1.2 \times 0.8$ & closed & 2.1 & $\begin{array}{l}20,0010- \\
50.0000\end{array}$ & $\begin{array}{l}65.000- \\
101,000\end{array}$ & $\begin{array}{l}\text { Göttingen } \\
417 \mathrm{~A}\end{array}$ & 2 & $\begin{array}{l}\text { KTH Mechan- } \\
\text { ics MTL }\end{array}$ & HWA \\
\hline Mexico $|17|$ & 4.5 & $9.5 \times 9.5$ & open & 17.6 & $\begin{array}{l}430.000- \\
560.000\end{array}$ & $\begin{array}{l}23 \mathrm{E} 6- \\
30 \mathrm{E} 6\end{array}$ & see [36] & 3 & DNW Open Jet & PIV, Forces, CP \\
\hline Myers $|46|$ & 0.1 & $1.35 \times 0.5$ & closed & 1.2 & - & 30.000 & - & $\begin{array}{l}\text { Porous } \\
\text { disc }\end{array}$ & $\begin{array}{l}\text { University of } \\
\text { Southampton }\end{array}$ & $c_{r} \cdot \operatorname{ADV}$ \\
\hline Neff $[4 \pi]$ & 0.3556 & $3.6 \times 2.4$ & closed & 1.1 & 33,000 & 220,000 & unknown & 3 & $\begin{array}{l}\text { Colorado State } \\
\text { EWT }\end{array}$ & Pitot tubes, $C_{r}$ \\
\hline
\end{tabular}


Table 2.1 - continued from previous page

\begin{tabular}{|c|c|c|c|c|c|c|c|c|c|c|}
\hline Author & Diameter & $\begin{array}{l}\text { Tunnel } \\
\text { Dimensions } \\
\text { [m] }\end{array}$ & $\begin{array}{l}\text { Test } \\
\text { Section }\end{array}$ & $\begin{array}{l}\text { Blockage } \\
{[\%]}\end{array}$ & $R e_{c}$ & $\operatorname{Ren}_{n}$ & Airfoil & Blades & Facility & Measurements \\
\hline NHEL $[48]$ & 10.08 & $24.4 \times 36.6$ & closed & 9.2 & 900,500 & $\begin{array}{l}3.3 \mathrm{E} 6- \\
17 \mathrm{E}^{-}\end{array}$ & $\mathrm{S} 809$ & 2 & NASA Ames & Forces. $C_{P}$ \\
\hline Whate $[49]$ & 0.175 & $0.4 \times 0.7$ & closed & 8.6 & $\begin{array}{l}6,400- \\
16.000\end{array}$ & 43,600 & flat plate & 2 & $\begin{array}{l}\text { University of } \\
\text { Edinburgh }\end{array}$ & PIV \\
\hline Xiao $[50]$ & 1.25 & $3.2(\mathrm{diam})$ & open & 15.3 & 175,000 & $1,000,000$ & $\$ 809$ & 2 & $\begin{array}{l}\text { China } \\
\text { Aerodynamics } \\
\text { Research and } \\
\text { Development } \\
\text { Center }\end{array}$ & PIV \\
\hline Yang [51] & 0.254 & $2.4 \times 2.3$ & closed & 0.9 & 14,000 & 67,000 & $\begin{array}{l}\text { MA0530TE } \\
\text { Prop }\end{array}$ & 3 & $\begin{array}{l}\text { Iowa State Uni- } \\
\text { versity } A B L\end{array}$ & PIV \\
\hline
\end{tabular}




\subsubsection{Single Rotor Experiments}

A significant number of experiments has focused on characterising the performance of a single. isolated wind turbine. The size of these rotor models - frequently on the order of $1 \mathrm{~m}$ - presents an opportunity to study blade loads, tip vortex development, and rotor performance. Measurements in the far wake are not frequently conducted with large rotor models, however, due to the size of the experimental facility that would be required. Tip vortex tracking downstream of two-bladed rotors has been conducted by several authors at the Delft University of Technology (TU Delft) in Refs. $[25,40,52]$ and tip vortices shed from three-bladed rotors were identified using PIV in Refs. [44.53]. One such experiment performed by Haans et al. used quantitative smoke visualisation to measure tip vortex core locations in axial and yawed flow conditions behind a $1.2 \mathrm{~m}$ diameter. two-bladed rotor in an open-jet wind tunnel [40]. The tip vortex core locations were identified and demonstrated good agreement with tip vortex traces that had been previously-recorded using a HWA with the same rotor [52]. The results presented in Ref. [40] established a linear relationship between the rotor thrust coefficient and the skew angle of the wake when the turbine was operated with $15^{\circ} .30^{\circ}$. and $45^{\circ}$ of yaw. Another recent experiment conducted by Akay et al. investigated the initial wake expansion behind a wind turbine in the $3 \mathrm{~m}$ diameter open-jet wind tunnel at TU Delft [25]. The tip vortex paths in the near wake of the turbine were identified in Ref. [25] with stereoscopic PIV and were compared to CFD predictions. The associated CFD computations were conducted with one domain that mimicked the experimental open-jet geometry and another that reflected freestream conditions and were designed to evaluate the effect of the tunnel geometry on the computed initial wake expansion. However, the results of the CFD computations were inconclusive.

Many wind turbine experiments have been conducted in water channels or flumes due to the beneficial increase in Reynolds number that these facilities can provide. Icing effects 
on wind turbine blades were investigated by Barber et al. in a water tow tank at ETH Zurich [54]. The three-bladed wind turbine used rotor blades that were $1 / 33$ scale versions of those used on the National Renewable Energy Laboratory (NREL) Phase VI turbine. The work evaluated the effect of various ice shapes along a rotor blade and identified that ice accretion on the outer $25 \%$ of a rotor blade is the most deleterious to wind turbine performance. A water channel experiment conducted in 2000 by Whale et al. recorded PIV measurements of the near wake behind a two-bladed wind turbine [49]. The tip vortex core locations identified through PIV demonstrated reasonable agreement with predictions from a free-wake computational code. Scaled marine turbine experiments performed by Bahaj et al. in a cavitation water tunnel and in a tow tank provide additional insight for testing wind turbine rotors in water [30]. Limits for cavitation on the rotor blades were established visually and the $C_{P}$ and $C_{T}$ curves were evaluated over a wide range of conditions. The marine current turbine was tested at two tip immersion depths $(0.55 \mathrm{D}$ and $0.19 \mathrm{D}$ ) in the tow tank in order to identify surface effects on the rotor performance. When the blade tip was $0.55 \mathrm{D}$ below the water surface. the power coefficient results closelymatched experiments in equivalent conditions in a cavitation tunnel. indicating that surface effects were not significant with the larger of the two tip immersion depths. When the blade tip was only $0.19 \mathrm{D}$ below the surface of the water, a reduction of $10-15 \%$ in $C_{P}$ was observed, however [30].

The NREL Unsteady Aerodynamics Experiment (UAE) Phase VI was performed throughout 1999 and 2000 using a 10 m diameter wind turbine installed in the NASA Ames $24 \mathrm{~m} \mathrm{x} 36 \mathrm{~m}$ wind tunnel and is the largest scaled wind turbine experiment conducted to date. A twisted. two-bladed, stall-controlled rotor was tested to characterise blade loads and rotor torque in an experiment with a Reynolds number that was representative of commercial-scale conditions. A summary of the experimental results can be found in Ref. [48] and a complete description of the test plan. model geometry, instrumentation. and 
calibration procedures can be found in Ref. [5j]. A blind code comparison was performed as part of the experiment with 30 researchers from 18 participating universities. government laboratories, and industrial partners in Europe and the United States. Participants were provided with the model geometry and test conditions, but did not have access to the test results until they had submitted their respective model's predictions. The codes that were tested ranged from blade element models. prescribed-wake models. free-wake models. and full Navier-Stokes codes [48]. The published results of the comparison demonstrated significant amounts of variability in prediction accuracy, little consistency between codes, and a limited ability to capture the aerodynamic effects occurring on the turbine $[48,56]$. In $0^{\circ}$ yaw. steady-state. unstalled conditions. the power predictions ranged from $25 \%$ to $175 \%$ of the measured data and at higher wind speeds, where dynamic stall and unsteady blade loading is present, the predicted power was $30 \%$ to $275 \%$ of the measured data [48]. Only one of the computational codes involved in the comparison accurately predicted the torque output of the rotor - the EllipSys3D incompressible Navier-Stokes solver from Risø [48]. However, each simulation with this particular computational code required days to perform and yawed- flow cases could not be considered as part of the comparison [48].

The NREL experiment produced a very valuable database that contained turbine loads and torque, but the flowfield near the rotor was not analysed. with the exception of qualitatively-observing tip vortices with smoke visualisation. In order to characterise the rotor flowfield in a wider range of flow conditions. The Modern Experiments in Controlled Conditions (MEXICO) wind turbine experiment was subsequently conducted in 2006 in the $9.5 \mathrm{~m} \times 9.5 \mathrm{~m} \mathrm{DNW}$ open-jet wind tunnel in the Netherlands. The three-bladed wind turbine was instrumented with pressure taps and load cells, and the wind tunnel was seeded with bubbles in order to conduct PIV of the rotor inflow and the near wake development. including tip vortex tracking [53]. The wind turbine rotor blades were composed of three airfoils that ranged from the DU91-W2-250 wind turbine airfoil near the root. to the RIS- 
A1-21 airfoil and finally to the NACA $64-418$ airfoil for the outboard $25 \%$ of the blade.

A more recent blind comparison study was organized by Krogstad and Eriksen in an attempt to evaluate the improvements that have been made in computational modelling since the NREL Phase VI experiment [57], 11 participants from 8 organisations were invited to deliver power and thrust curves along with the wake velocity deficit and turbulence kinetic energy predictions at downstream planes of $X / D=1,3$, and 5 . The rotor that was studied was a $0.894 \mathrm{~m}$ diameter, three-bladed wind turbine and the rotor blades used the NREL S826 wind turbine airfoil. The speed in the wake had been determined with a cross-wire hot-wire probe. The codes that were used for the blind predictions ranged from blade element momentum theory (BEMT) computations to Large Eddy Simulation (LES). The results indicated that the $C_{P}$ curve of the rotor was predicted to within $\pm 10 \%$ of the measurements and that the thrust coefficients were consistently underpredicted by the majority of the codes [57]. The velocity deficit predictions in the wake at three downstream planes typically failed to capture the asymmetry that was observed downstream of the rotor and the particularly large tower effects in this experiment [57]. The computations conducted with RANS solvers typically had as much variation from the experimental results as LES predictions. Despite some large errors in the predicted turbulence kinetic energy. the results of this blind comparison study more closely matched the experiment when compared to the earlier NREL Phase VI blind comparison, and highlights the progress that has been made in modelling stall and spanwise flow along wind turbine blades. The blind comparison study built on preliminary studies using the same rotor that assessed the thrust and power characteristics of the $0.894 \mathrm{~m}$ turbine [58]. The near wake profile in axial and yawed flow was recorded using pitot tubes and a laser doppler anemometer in this earlier study [58].

While the blind comparison organised by Krogstad measured velocity profiles up to 5D downstream of a $0.894 \mathrm{~m}$ rotor, far wake measurements typically necessitate a smaller rotor model. A detailed study of wind turbine wake velocities and turbulence intensities was 
conducted by Chamorro and Porté-Agel in which the evolution of the wake downstream of a $15 \mathrm{~cm}$ diameter wind turbine was characterised [16.34]: These measurements were recorded in the atmospheric boundary layer wind tunnel at the Lniversity of Minnesota is St. Anthony Falls Laboratory. Wake effects were characterised in neutral and stable boundary layer conditions [16.34]. Measurements recorded in a neutral boundary layer investigated roughness effects on the downstream wake development using a cross-wire hot-wire probe. A velocity deficit was still observed $15 \mathrm{D}$ downstream of the turbine and larger velocity deficits were typically present in smooth boundary layer conditions at each downstream plane [16]. Stable boundary layer conditions were subsequently created in an atmospheric boundary layer wind tunnel using cooled metal plates to create buoyant, or stratified conditions [34].

An alternative to a dynamic rotor model in a wind turbine experiment is the use of a static mesh simulator. These can take the form of a porous metal disc or a wire mesh that will experience a thrust coefficient similar to that imposed by a rotating model. Aubrun et al. compared the wake generated by a $0.416 \mathrm{~m}$ diameter. three-bladed wind turbine model with a wire mesh disc of the same diameter and characterised the wake using two single-normal hot-wire probes [29]. The study established that the lateral wake meandering phenomenon downstream of a turbine occurred downstream of both types of models and that a static mesh simulator was sufficient to generate a representative velocity deficit in the far wake [29]. Earlier work by the same authors in 2007 evaluated the relationship between the porosity level in a disc, the thrust coefficient, and the velocity profile in the wake [28]. Medici and Alfredsson also compared the wake behaviour downstream of a porous disc to the wake generated by a two-bladed wind turbine in 2005 [45]. The disc and turbine were operated at the same Reynolds number based on the freestream speed and the rotor diameter and flow measurements were recorded with a cross-wire hot-wire probe. The authors identified that large-scale vortex shedding differed between the static and rotating models [45]. The authors also observed evidence of tip vortices far downstream 
in the wake. at $X / D=9$ whereas tip vortices are typically assumed to dissipate within $3-4 D$ of the rotor. Myers and Bahaj also explored the use of porous discs to mimic the behaviour of marine current turbines at the University of Southampton [59]. The work identified that the downstream velocity deficit was predominantly dependent on the turbulence intensity in the water flume [59].

\subsubsection{Blockage Effects}

A particular concern in scaled wind turbine experiments is the effect of blockage on the downstream wake development. Blockage effects are caused by the confinement of the flow due to the presence of the test section walls. This will cause artificial flow acceleration around an object to preserve continuity and has a tendency to straighten out the streamlines travelling a model. Additionally, blockage will lead to recorded forces and moments which are larger than they would be if the same model was tested in the absence of walls. In general, blockage in a wind tunnel or water channel environment is characterised by the level of solid blockage occurring in the experiment. Solid blockage effects can be estimated based on the frontal area of the solid (and generally stationary) object being tested and any supporting structure near the measurement plane. Solid blockage in the context of a wind turbine experiment would be based on the frontal area of the turbine blades, the hub, and the tower. This definition, however. would underestimate the effective blockage caused by the dynamic motion of the rotor and the associated wake blockage. A more appropriate and more commonly applied definition of blockage for wind turbine experiments is based on the swept area of the rotor. The definition of blockage shown in Equation (2.10) that is characterised by the ratio between the rotor swept area and the cross-sectional area of the test section $\left(A_{x}\right)$ will be used throughout the thesis. 


$$
\text { Blockage }=\frac{A_{\text {swept }}}{A_{x}} \times 100
$$

Excessive amounts of blockage will cause artificial flow acceleration around an object in a closed test section which can modify the development of downstream flow structures. On the other hand. large amounts of blockage in an open-jet test section may cause the expanding wake and the mean flow field to slow down artificially. This is a concern as the factors influencing the wake expansion in wake interference experiments should be limited to the surface representing the ground, neighbouring wakes, the turbulent inflow conditions. and atmospheric stability. The extent of the blockage in a number of existing wind turbine experiments conducted in closed and open-jet test sections has been compiled in a histogram in Figure 2.7. If both closed and open test sections are considered, the blockage ratio within this sample of approximately two dozen experiments ranges from $1 \%$ to $53 \%[16,25.30,32$. 35,37 . Figure 2.7 also demonstrates that the majority of existing experiments have been conducted with blockage greater than $10 \%$. Given the wide range of blockage observed in literature, an understanding of blockage effects on the initial wake expansion is desirable in order to more carefully consider wake interference studies.

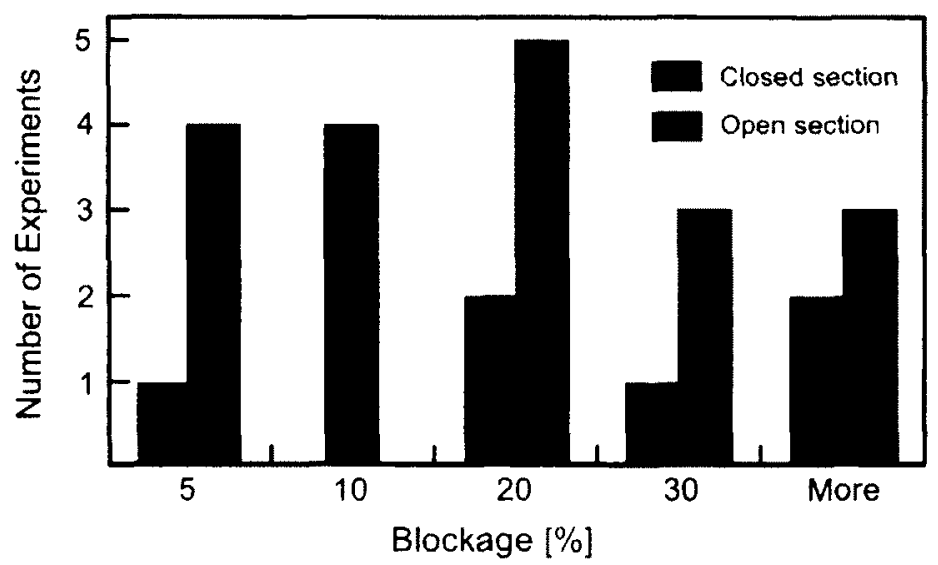

Figure 2.7: Distribution of blockage in existing experiments. Data derived from Refs. $[15-17.23 .25-27,30,32.35 .37 .39 .40 .42 .44 .47 .49 .50 .54 .56]$. 
Analytical blockage corrections are the standard way of correcting thrust and power coefficients [30.60]. These differ from typical wind tunnel blockage corrections highlighted by Pope as a wind turbine is extracting energy from the flow [61]. A commonly used blockage correction for wind turbines is derived in Ref. [30]. The correction, which will be described in Chapter 3, is solved iteratively and is dependent on the thrust coefficient of the wind turbine. the turbine diameter. and the test section cross-sectional area. These corrections were applied extensively by Burdett and Van Treuren as part of an experiment with several scaled wind turbines with blockage that ranged from $19 \%$ to $53 \%$ [32]. The work of Burdett and Van Treuren demonstrated that blockage corrections could adequately correct recorded power coefficients even at the very high blockage of $53 \%$. Despite their ability to account for the effects of blockage on power and thrust coefficients, corrections are seldom implemented in literature when blockage approaches $10 \%$. Experiments by Barber et al. [54], Adaramola and Krogstad [23], Bartl [62] and the NREL Phase VI experiment [48] had blockage that ranged from $7.1 \%$ to $13.5 \%$ and did not apply blockage corrections. The accuracy of neglecting blockage effects on the recorded thrust and power coefficients will depend primarily on the thrust coefficient under consideration based on the blockage correction model of Bahaj et al. [30].

Chen and Liou determined the blockage factor required to correct thrust and power data experimentally in a wind tunnel in 2011 [35]. This was accomplished by recording the centreline speed $1 \mathrm{~m}$ upstream of the rotor plane with and without a wind turbine installed in the test section. Three separate rotors were used with blockage that ranged from $10.2 \%$ to $28.3 \%$. The authors confirmed that the blockage correction factor was dependent on the rotor diameter. The required correction was also dependent on the tip speed ratio of the turbine and the blade root pitch angle. as each of these parameters will affect the thrust generated by a turbine. The correction factors that were determined experimentally had a similar magnitude to the analytical corrections implemented by Bahaj et al. [30]. While 
this reinforces the applicability of the analytical corrections, the effect of blockage on the development of the wake was not considered in Ref. [35].

A detailed investigation into tunnel blockage effects created by small vertical axis wind turbines (VAWT) was presented in 2011 by Ross et al. [63]. and identified a tolerable blockage limit of $3.8 \%$ before the recorded power coefficients were influenced by the VAWT and its asymmetric wake. The effect of wind tunnel blockage on the expansion of the wake downstream of scaled horizontal axis wind turbines in closed test sections, however, has not been treated explicitly in literature, to the author's knowledge.

\subsubsection{Reynolds and scaling}

Despite the increasing number of wind turbine experiments available in literature, scaling effects are still not well understood. Several recent measurement campaigns were conducted with Reynolds numbers based on the blade tip speed and tip chord $\left(R e_{t i p}\right)$ on the order of $10^{3}$ to $10^{4}[16,26,35,44,47,49]$ and additional medium-scale experiments have considered Reynolds numbers ranging from $10^{5}$ to $2 \cdot 10^{5}[23,30,38,40,54]$.

The large-scale MEXICO and NREL UAE Phase VI turbines were tested at Reynolds numbers that ranged from $5 \cdot 10^{5}$ to $10^{6}[17,56]$. Several recent experiments have considered scaled versions of full-scale experimental wind turbines. The experimental campaign presented by Cho and Kim assessed the performance of a $1 / 2.25$ geometrically-scaled version of the MEXICO rotor [36]. That medium-scale experiment measured the torque output of the rotor when the rotor blades were operating in free laminar-to-turbulent transition and forced transition to turbulence conditions. The torque coefficient was dependent on the transitional behaviour of the airfoils along the rotor blade span and on the Reynolds number based on the tip conditions, but a comparison was not made with full-scale results [36]. Xiao et al. used PIV to evaluate the initial wake expansion downstream of a $1 / 8$ scaled version 
of the NREL UAE Phase VI rotor [50]. The initial wake expansion was assessed using the tip vortex core locations. but could not be compared to the full-scale experiment. as wake expansion was not quantified in the full-scale campaign [56]. Additionally. experiments presented in Refs. [31.54] measured the rotor performance and velocities in the wake behind three-bladed wind turbines that used 1/33 scale versions of the NREL UAE Phase VI rotor blades. The power measurements recorded in the scaled test by Barber et al. were corrected for Reynolds number effects and demonstrated good agreement with the fullscale NREL values [54]. A set of experiments conducted by Burdett and Van Treuren tested the performance of wind tunnel models that had been dimensionally-scaled while maintaining Reynolds similarity [32]. The power curves generated by the three tested rotors with different diameters but at consistent Reynolds numbers demonstrated consistent performance [32].

One of the most comprehensive assessments of Reynolds number effects in literature was recently published in 2012 by Chamorro et al. [64]. The authors evaluated the mean speed and turbulence statistics in the wake of a $15 \mathrm{~cm}$ diameter wind turbine using a cross-wire hot-wire probe. The Reynolds numbers that were considered in the experiment ranged from 16.000 to 173.000 based on the speed at hub height and the turbine diameter $\left(\operatorname{Re}_{D}\right)$. The authors found that the mean velocity profile became independent of Reynolds number when $R e_{D} \approx 48.000$ [64]. Additionally, the turbulence statistics became independent of the Reynolds number when $\operatorname{Re}_{D}$ was at least 93,000 [64]. The dependence of the rotor loads on the Reynolds number was not identified by Chamorro et al. . however [64].

Although a formal scale classification for the above-mentioned experiments does not exist, in the current work. small-scale refers to experiments where the tip Reynolds number is on the order of $10^{3}$ to $10^{4}$ and medium-scale refers to experiments where the Reynolds number is on the order of $10^{5}$. The MEXICO and NREL UAE Phase VI experiments are classified here as large-scale experiments. The Reynolds number based on the tip speed 
and tip chord of a number of experiments is plotted with respect to rotor diameter in Figure 2.8. highlighting the range of scales that has been considered in experiments to date. Representative data for several commercial-scale horizontal axis wind turbines have been included in Figure 2.8 and these wind turbines have a Reynolds number on the order of $10^{6}$ and diameters that range from $80 \mathrm{~m}$ to $160 \mathrm{~m}$. The Reynolds number range considered in the current study varies from 3,620 to 47,000 and this range is compared to existing experiments in Figure 2.8. It has been discussed in Refs. [65] and [66] that the Reynolds number will have a minor influence on the wake behaviour above a lower limit of approximately 1.000 . based on computational analysis of the inflow behaviour of the rotor using an actuator disc computational method. It was, however. desired to experimentally-evaluate the effect of the Reynolds number on the initial wake expansion downstream of a model rotor to gain an improved understanding of the potential limitations of scaled wind turbine experiments.

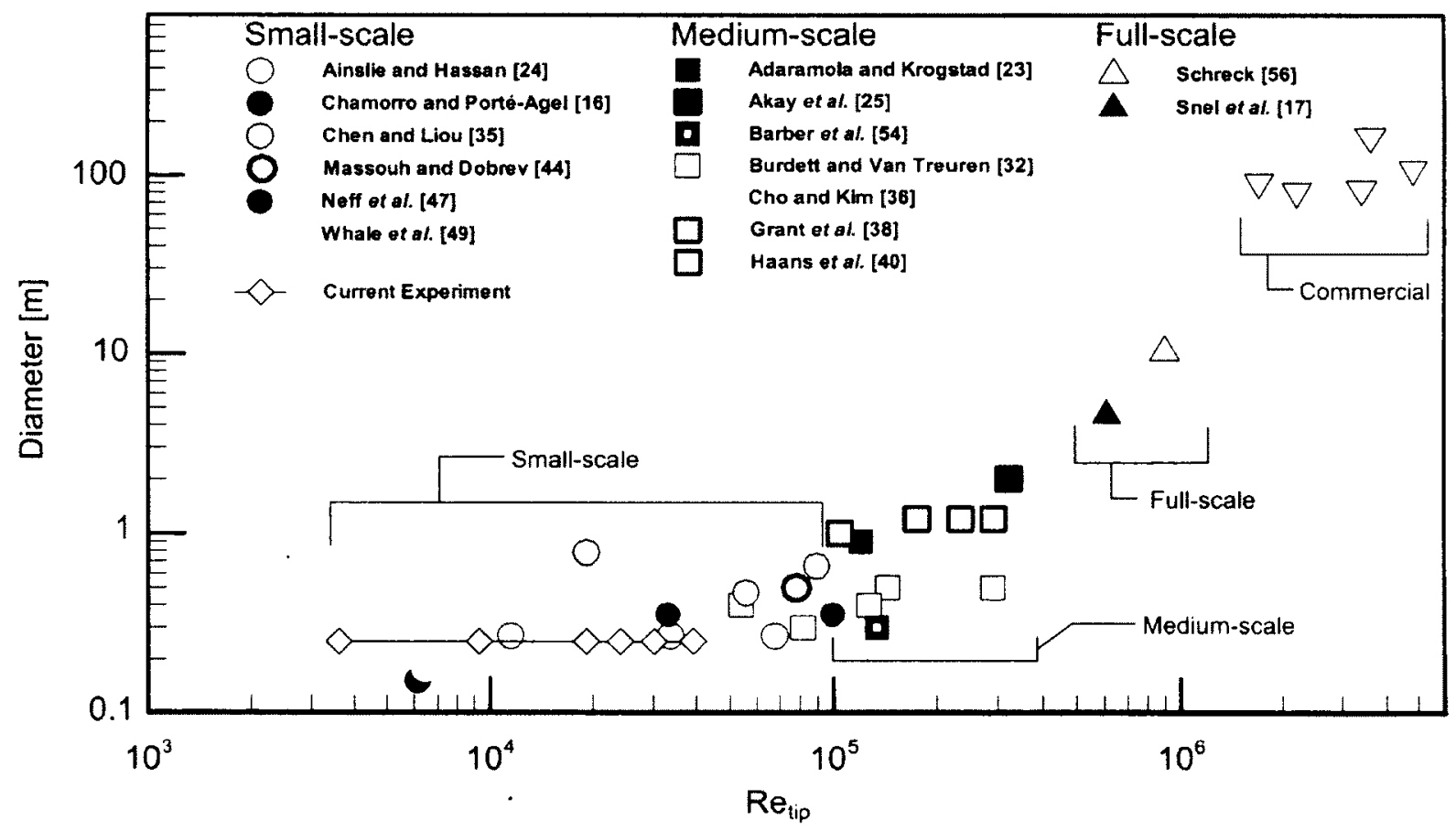

Figure 2.8: Summary of the Reynolds numbers and rotor diameters in existing experiments. 


\subsubsection{Wake Interference Effects}

The improved understanding of wake development downstream of single rotors has led to a number of wake interference experiments. These wake interaction studies have predominantly focused on understanding the effect of an upstream turbine wake on the power deficit experienced by a longitudinally-aligned. downwind rotor. One such experiment was conducted by Maeda et al. using two rotors with longitudinal separation [42]. The longitudinal separation between the rotors was varied from 3D to $10 \mathrm{D}$ and the downstream rotor was also given a lateral offset ranging from $\pm 2 \mathrm{D}$ from the centreline. The study was designed to measure the blade loads in full-wake and partial-wake flow conditions. Several wake effect characteristics were identified in Ref. [42] including the presence of an asymmetric wake, and that adjusting the location of downstream rotor by $\pm 1.5 \mathrm{D}$ from the centreline removed the rotor from the upstream wake effects. Fluctuations in blade flapping moments and tower moments were observed to be a maximum when the downstream rotor was offset from the centreline by $0.25-0.375 \mathrm{D}$. The study did produce uncharacteristically large power losses at the downstream rotor. however, which were $85 \%$ at a downstream distance of $3 \mathrm{D}$ and were still $65 \% 10 \mathrm{D}$ downstream [42].

Recent experiments conducted in the water tow-tank at ETH-Zurich by Barber et al. and Tsalicoglou et al. evaluated various interference effects using two longitudinally-aligned turbines [31.67]. In the first instance, the wake speed downstream of the upstream rotor was determined in uniform flow using an acoustic doppler velocimeter (ADV). Barber et al. identified that the measured wake profile differed from the typically-assumed Gaussian. axisymmetric profile implemented in wake models. The possible errors in predicting the annual energy output of a wind farm that result from typical wake model assumptions were subsequently evaluated using a BEMT model in Ref. [31]. The authors identified that the assumption of a purely Gaussian velocity profile led to higher errors than other 
BEMT-related assumptions when computing the annual energy production of a wind farm. A separate experiment conducted by Tsalicoglou et al. in the same tow tank investigated the effects of the wind inclination angle due to terrain effects on wind farm performance using two rotors [67]. The experimental setup was designed to mimic inclined wake effects that occurred between two, longitudinally-aligned but vertically-separated wind turbines installed on a hill. The downstream separation varied from $3 \mathrm{D}$ to $6.5 \mathrm{D}$ and the flow inclination angle of $15^{\circ}$ was achieved by tilting the rotors in the water channel. The study demonstrated that a downstream rotor in axial flow generates $45 \%$ less power than the upstream rotor, but that the drop in power was less significant in inclined flow due to the deflection of the wake by the upstream rotor [67].

A comprehensive wake interference study was conducted by Adaramola and Krogstad using two $0.9 \mathrm{~m}$ diameter wind turbines with a longitudinal offset that was varied from 3D to $9 \mathrm{D}$ [23]. The work conducted in Ref. [23] evaluated the effect of the operating conditions of the upstream rotor on the downstream rotor's performance, including the effects of tip speed ratio, yaw angle, and blade pitch angle. The performance loss of a downstream turbine was up to $45 \%$ compared to the upstream turbine in a longitudinallyaligned configuration. The authors identified that the overall array efficiency of the two turbines could be increased by modifying the yaw angle of the upstream rotor in order to partially deflect the wake. An alternate method of increasing the array efficiency was by either changing the tip pitch angle or the tip speed ratio of the upstream turbine in order to reduce its thrust coefficient. This approach reduced the losses of the downstream turbine by $5 \%$ to $10 \%$ [23]. Two wind turbines with similar diameters to the experiment presented by Adaramola and Krogstad were used in an experiment presented by Bartl in 2011 [62]. The upstream rotor in Ref. [62] had a diameter of $0.94 \mathrm{~m}$ and the downstream rotor had a diameter of $0.9 \mathrm{~m}$ and both turbines had a NREL S826 airfoil. The performance of the downstream turbine was evaluated with longitudinal separation distances of $3 \mathrm{D}$ and $5 \mathrm{D}$. 
In addition to recording power and thrust data. Bartl also characterised the wake velocity profiles using a single-normal hot-wire probe [62].

Meyers and Meneveau investigated optimal turbine spacing distances of very large wind farms using an optimisation model that was refined using LES of a large wind farm [68]. In very large wind farms (on the order of $10 \mathrm{~km}$ long), the surface roughness created by the scattered wind turbines will modify the boundary layer profile and will lead to a decrease in the hub speed far downstream. Meneveau and Myers evaluated the equilibrium boundary layer that developed over a variety of wind farm configurations and developed a cost ratio for optimisation that included the costs of land use and the cost per turbine in the farm [68]. Based on their work, the authors suggested that an optimum longitudinal spacing distance between turbines that considers the boundary layer development as well as land use and turbine costs may be approximately $15 \mathrm{D}[68]$.

Despite the increasing number of wake interaction studies available in literature. the lateral spacing between wind turbines within a row has not been thoroughly evaluated. The hub-to-hub spacing between wind turbines in a wind farm has historically been on the order of $5 \mathrm{D}$ to $7 \mathrm{D}$ for irregular, onshore wind farms. Recent wind farms have been installed with hub-to-hub separation distances of $3.1-4.5 \mathrm{D}$ in the predominant wind direction. however $[11,69]$. Hossain et al. examined the use of a wall of microturbines in order to provide a windbreak for agricultural or urban purposes while still generating electricity [41]. A $5 \times 5$ vertically-mounted array of $5 \mathrm{~cm}$ diameter. four-bladed. wind turbines was tested in a wind tunnel with a $0.2 \mathrm{D}$ gap width between blade tips. Downstream HWA and PIV measurements demonstrated that wake recovery was significantly delayed downstream of the array compared to the wake recovery downstream of a single rotor [41]. The gap between blade tips in the previously-described static mesh simulator wind farm was 2D. but lateral interference effects were not noted [28]. As part of a marine current turbine study. Bahaj et al. tested the effect of a lateral tandem rotor arrangement with a gap width that ranged 
from $0.5 \mathrm{D}$ to $0.0125 \mathrm{D}$ [30]. Interference effects from one rotor to the main test rotor were not apparent in Ref. [30]. however. A recent marine current turbine experiment conducted by Myers and Bahaj investigated the use of two-row arrays using porous metal discs to represent the effect of marine turbines [46]. Two upstream turbines were arranged with a $1.5 \mathrm{D}$ gap between the discs and a third. downstream rotor was located $3 \mathrm{D}$ downstream. The work presented in Ref. [46] indicated that the thrust coefficient of the central disc could be increased due to accelerated flow through the upstream gap. In addition to the work of Myers and Bahaj, the tidal turbine community has recently been investigating the concept of a tidal fence, or a linear array of tidal turbines across a partially blocked channel using computational methods in order to improve the yield of these turbines [70-73].

\subsubsection{Scaled wind farms}

In order to extend the knowledge gained from two-rotor wake interference experiments and to improve the fundamental understanding of wake development in a wind farm, several researchers have conducted scaled wind farm experiments. The earliest example of an experiment with a scaled wind farm configuration in a wind tunnel was conducted by Ainslie et al. in 1989. The experiment used three-bladed wind turbines and was used to validate a novel wake model $[22,24]$.

Despite the knowledge gained as a result of the work by Ainslie et al., additional scaled wind farm experiments have only appeared in literature since 2004. Aubrun conducted experiments on a scaled wind farm composed of 9 static mesh discs in flow conditions representative of an offshore ABL [28]. The discs were arranged in a $3 \times 3$ grid configuration with $3 \mathrm{D}$ longitudinal and lateral separation. The speed in the wakes of the discs was determined using a triple hot-fibre probe and the thrust coefficients of the turbine simulators were back-calculated by integrating the velocity deficit. The authors remarked that the 
decreased wake speed downstream of the first row of turbines resulted in a decrease in the power coefficient of approximately $60 \%$ at the second row of discs. but that the third row experienced similar output to the second row [28].

A similar wind farm configuration was evaluated at Carleton University using 9 scaled wind turbine models [74]. The wind farm was arranged in a $3 \times 3$ grid with $2 \mathrm{D}$ lateral separation and 5D longitudinal separation between rows. The electrical power output of the turbines was calculated and demonstrated that the power output in the second row of turbines was reduced by $45 \%$ compared to the upstream row. The power output in the third row of turbines was similar to that of the second row and followed the expected behaviour also noted in the experimental work of Aubrun et al. and in the full-scale measurements at the Horns Rev wind farm $[10,28]$.

Two scaled wind farm experiments published in 2011 by Chamorro et al. and Chamorro and Porté-Agel investigated grid-aligned and staggered wind farm configurations [75.76]. The first of these experiments arranged $30.15 \mathrm{~cm}$ diameter wind turbines in a $10 \times 3$ grid. with $4 \mathrm{D}$ lateral separation within a row and 5D or 7D longitudinal separation between the 10 rows [75]. Velocity profiles were measured along the centreline of the middle column in the wind farm using a cross-wire hot-wire probe and were observed to reach an equilibrium condition $4-5$ rotors into the wind farm. Tip vortex signatures were also observed 5 7D downstream of the turbines. The authors identified that very large wind farms may be idealized as a case of surface roughness with a transition region followed by a fullydeveloped region [75]. Subsequent work by the lead author investigated a 10-row wind farm where the rows contained alternating numbers of turbines (either 2 or 3 ) in a staggered arrangement [76]. Turbines were separated by $4 \mathrm{D}$ in the lateral direction and adjacent rows of turbines were separated longitudinally by $5 \mathrm{D}$. The authors identified that the staggered arrangement produced $10 \%$ more power than the grid-aligned placement of turbines by effectively increasing the wake recovery distance and the momentum transfer to the wake 
between successive longitudinal turbines. It was also observed in Ref. [76] that the internal boundary layer that forms over the wind farm developed more slowly with a staggered arrangement compared to a grid-like configuration.

Several compact wind farm configurations were evaluated by Ozbay in 2012 in the 2.4 $\mathrm{m} \times 2.3 \mathrm{~m}$ atmospheric boundary layer wind tunnel at the Iowa State University [77]. The author evaluated a number of wind turbine configurations using $0.254 \mathrm{~m}$ diameter turbines, including a $3 \times 3$ grid of turbines with $3 \mathrm{D}$ lateral separation and $3 \mathrm{D}$ longitudinal separation, a 3-2-3 staggered configuration with 3D lateral separation in which the middle row's turbines were offset, as well as the effect of a $2 \mathrm{D}$ ridge on longitudinally-aligned turbines, and deep array effects using 5 longitudinally-aligned turbines with 3D downstream separation. It was demonstrated that the array efficiency of the staggered configuration was $20 \%$ to $24 \%$ better than the grid configuration with the same downstream separation. The staggered configuration created a larger effective downstream wake recovery distance in between successive, aligned turbines. The gains observed with a staggered configuration in this particular work were larger than those observed by Chamorro et al. in a similar experimental setup [75]. This occurred due to the fact that the wind farm losses in the baseline configuration were much larger in the experiment presented by Ozbay due to 3D longitudinal separation, compared to 5D separation in the work of Chamorro et al. [75.77].

A comprehensive wind tunnel campaign was undertaken by Corten et al. using up to 28 scaled wind turbines [78.79]. The two-bladed wind turbines had a diameter of $0.25 \mathrm{~m}$. a tip Reynolds number of approximately 25.000 and operated at a tip speed ratio of 5.5 . The rotors were scaled aerodynamically and this resulted in a proportionally larger chord. The wind turbines were operated in the $3 \mathrm{~m} \times 2 \mathrm{~m}$ atmospheric boundary layer tunnel at TNO in the Netherlands in representative offshore boundary layer conditions. Grid configurations consisting of $8 \times 3,14 \times 2$, and $4 \times 7$ turbines were evaluated, along with a staggered configuration. The lateral hub-to-hub spacing between turbines within a row was 
typically $3 \mathrm{D}$ and the longitudinal separation between rows was typically $4 \mathrm{D}-5 \mathrm{D}$. although the exact separation distances varied between configurations [79]. A separate configuration was evaluated using a single row of three turbines with $2.5 \mathrm{D}$ lateral separation between turbine towers. The authors noted that interference effects between the laterally-arranged turbines were not observed [79].

\subsubsection{Increasing wind farm performance}

A number of novel approaches to improving wind farm performance have recently been investigated. The concept of reducing wake losses in offshore wind farms through upscaling wind turbines was investigated as part of the UpWind research program in the European Union (EU) [80]. The authors assessed the wake losses that would occur with $100.5 \mathrm{MW}$ wind turbines compared to the losses that would be incurred with 25, 20 MW turbines installed within the same area. The $20 \mathrm{MW}$ wind turbine was a hypothetical design with a $252 \mathrm{~m}$ diameter and the wake effects were assessed using three computational wake models. The upscaling study indicated that gains of $6.5 \%$ to $14.5 \%$ could be acheived by installing fewer, larger, wind turbines over a given surface area [80].

Another unconventional method of increasing wind farm performance involves controlling the orientation of the turbine wake by modifying the yaw angle of the upstream turbine in a wind farm. The upstream turbine will experience a decrease in performance and increased unsteady loading, but the farm efficiency will increase as the wake can be partially-deflected away from downstream turbines $[80]$.

A third approach to improving array efficiency has been independently evaluated by Corten and Schaak [78] and Adaramola and Krogstad [23]. This approach involves deliberately reducing the power generated by the upstream turbine in a column of wind turbines either by changing its blade pitch angle or by altering its tip speed ratio. This 
results in a lower induction factor, less energy extraction. and reduces the velocity deficit downstream of the first rotor. Subsequent turbines have been shown to generate more power and experience lower axial forces [78]. Corten and Schaak achieved this effect by changing the blade pitch angle of the upstream turbines in a wind farm. Experiments confirmed that the array efficiency of a 24 turbine wind farm could be increased by $2-4.5 \%$ and that axial loading of the turbines could be reduced by $20 \%$ by adjusting the upstream turbine's blade pitch angle [78]. In an experiment involving two wind turbines. Adaramola and Krogstad identified that the array efficiency of the two longitudinally-aligned rotors could be increased by $5-7 \%$ by adjusting either the tip speed ratio or the tip pitch angle of the upstream rotor [23].

\subsection{Computational Approaches}

Computational simulations can be implemented as an alternative to experiments in order to evaluate wind turbine performance and wake effects. The range of currentlyused techniques consists of blade element methods. wake models. actuator disc methods. prescribed- and free-wake methods, and CFD methods. An early review of aerodynamic modelling efforts for wind turbines was given by Snel in 1998 [81] with a follow-up review in 2003 [82]. A thorough review of the aerodynamic techniques that are used to resolve the near- and far-wakes of wind turbines was published by Vermeer et al. in 2003 [19]. A follow-up review in 2006 by Hansen et al. summarized the aerodynamic and aeroelastic techniques that are presently being used to evaluate wind turbine loads and a more recent review by Sanderse addressed techniques used to evaluate wind turbine wakes $[21,83]$. 


\subsubsection{Blade Element Momentum Theory}

Blade element momentum theory (BEMT) methods are the simplest and most commercially used analysis tools available for wind turbines [84]. They use a combination of blade element theory and momentum theory along with a series of empirical approximations to calculate the loads acting on a turbine blade at a discrete number of radial elements. This theory has been developed from the blade element theory of Glauert in 1935 [85]. A detailed derivation of BEMTs based on modeling the axial and radial inflow can be found in Refs. [86,87], whereas a BEMT based solely on the axial inflow to the rotor disc has been described by Leishman [88]. A review of the application of BEMT techniques in commercial wind turbine analysis software is presented by Molenaar and Dijkstra in [84]. While BENTs have been shown to reasonably predict inflow behaviour within a range of axisymmetric conditions [89]. they cannot characterise stalled flow conditions and require mathematical corrections at typical tip speed ratios [88]. Additional corrections that account for tip losses. root losses, yaw effects. and spanwise flow can be implemented to improve the fidelity of BEMT models [87.90]. Despite the relative simplicity of BEMTs. their rapid computation time makes them particularly well suited for assessing the forces and torque generated by new rotor designs.

\subsubsection{Wind Turbine Wake Modeling}

A rapid method of assessing wake effects in wind farms is through the use of wake models. A summary of currently-used wind farm tools was given in 2001 by Schepers et al. [91] and a detailed description of early wake modeling efforts was provided by Crespo [92]. The three types of wake models can be classified as roughness models. kinematic models, and field models [92]. The earliest methods for evaluating wind turbine wakes treated wind turbines as distributed roughness elements that affected the mean flowfield. 
Kinematic models assume axisymmetric self-similar wake velocity profiles and typically relate the velocity deficit to the thrust coefficient of the turbine [92]. The first kinematic model for wind turbine wakes was developed by Lissaman [93]. but other models were developed in the 1980s by Katic et al. [94] and Voutsinas et al. [95]. Field models calculate the flow properties at all of the designated points in a simplified flow field and use simplified Navier-Stokes equations with a 0-, 1-, or 2-equation turbulence model [92].

Wake models have been validated against a variety of data sets in wind tunnels and in the field. The GH Eddy Viscosity wake model has been validated against a wind tunnel experiment performed by Ainslie and Hassan in $1989[24,96]$. The results of the wind tunnel tests demonstrated that the wind speed profiles across the wake behind a single turbine are well predicted where the velocity deficit has an approximately Gaussian shape. The Ainslie model was also validated against power measurements taken at the Nørrekær Enge II wind farm [96]. Though qualitative agreement was demonstrated between the model and the measured power, a difference of $25 \%$ was observed between the power predictions and the measurements at each row of turbines [96].

The ENDOW and UpWind research programs in the EU compared the wake predictions of several wind farm tools with experimental measurements at offshore wind farms. The ENDOW program studied the wake measurements from the Vindeby offshore wind farm in 2001 where it was observed that model errors downstream of a single rotor were within $\pm 20 \%$ of the measured data [97]. The previously-mentioned UpWind program studied wake model predictions at the Horns Rev and Nysted offshore wind farms and demonstrated improvements in the capabilities of existing wake modelling tools compared to the earlier ENDOW program $[10.80,98]$. A number of wake models were validated using experimental and commercial-scale data by Renkema in 2007 [99]. Despite the recent improvements in wake modelling capabilities. a number of models rely on empirical constants and may have to be tuned to match conditions at a certain site. 


\subsubsection{Prescribed- and Free-wake Methods}

Potential flow methods for wind turbines can be classified as prescribed-wake methods or free-wake methods. In prescribed-wake methods. the shape of the wake is predefined and does not deform as a result of self-induced velocities. In free-vortex methods. however. the wake is created by successive vortex element shedding and wake deformation is determined by the self-induced velocities of the vortex elements as well as the induced velocity of the rotor blades [100]. Prescribed-wake methods have been used by several authors [100-104]. whereas free-wake methods have not been as commonly used to analyse wind turbine wakes $[49,89,105]$

In 2003, Duque et al. tested a free-wake vortex lattice code. CAMRAD II. originally developed for helicopters, against the NREL Phase VI measured data [105]. Results for CAMRAD II with a stall delay model were within $10 \%$ of the measured power until the freestream speed increased beyond $15 \mathrm{~m} / \mathrm{s}$, after which a $50 \%$ over-prediction of power was observed [105]. A more recently-developed free-wake vortex code was developed at the University of Maryland by Gupta and Leishman [106]. Each blade was modelled using a lifting surface and a single vortex filament was trailed from the tip of each blade. The results of this work have been validated in a series of papers in axial and yawed conditions [89.107-109]. The numerical results of this code are promising. though the inadequacies in stall delay models and two-dimensional polar data have been discussed in Ref. [106].

A particular class of free-vortex methods are vortex particle methods (VPM), in which the distributed vorticity in a flow is represented by a discrete number of particles of a finite size. The GENUVP code. developed by Voutsinas et al., models unsteady. incompressible. inviscid. and rotational flows using vortex particles [110-112]. A vortex particle approximation (also referred to as vortex blobs in literature) of the free vorticity is used to simulate the behaviour of the wake and a dipole approximation is used for the 
flow induced by the turbine blades. Vortex particles are shed from spanwise strips along the trailing edge of a blade as well as from the chordwise elements along the blade tip. An unsteady computational code created by Willis et al. uses a similar vortex particle formulation to model the wakes of fixed and flapping aircraft wings [113].

GENUVP forms the aerodynamic basis for SmartRotor. the aeroelastic discrete vortex method developed at Carleton University in 2002 [114]. SmartRotor is an aeroelastic. aeroacoustic code for rotorcraft and wind turbine analysis and combines a VPM to simulate the wake, a nonlinear structural dynamics model to simulate blade deflection. and an acoustic model. SmartRotor has been used to properly characterise several aeroelastic helicopter and wind turbine problems and has been validated against available data for the NREL Phase VI and Tjæreborg wind turbines [20,115-119]. The wake generated by the code demonstrated good agreement with vorticity transport method computations performed by Fletcher et al. [120].

Prescribed- and free-wake methods typically use a panel method representation for the blades and rely on two-dimensional airfoil data. dynamic stall models, and stall delay models and therefore have difficulty in accurately resolving blade loads under stalled conditions [20]. Despite these challenges. a vortex particle representation of the wake is highly advantageous as the approach does not suffer from the numerical dissipation that is present in grid-based RANS solvers. Similarly, the grid-free nature of vortex particle codes eliminates the need for the very sophisticated grid techniques required in rotary-wing grid-based CFD.

\subsubsection{Navier-Stokes Computational Fluid Dynamics}

There have been a number of RANS CFD simulations conducted over the past decade and they have demonstrated a clear ability to resolve blade loads and rotor torque. RANS CFD has also been used to adequately predict the forces and rotor torque generated by 
small vertical axis wind turbines by McTavish et al. and Jaohindy et al. [121.122]. RANS CFD simulations, however. can suffer from numerical dissipation in the turbine wake that can limit their applicability for characterising the downstream wake.

The first instances of the application of RANS CFD techniques to model complete wind turbines were conducted by Sørensen and Duque in 1998 and 1999, respectively [123. 124]. Sørensen used an early version of EllipSys3D, an incompressible Navier-Stokes solver code developed at Risø. to simulate a Nordtank $500 \mathrm{~kW}$ 3-bladed wind turbine using a 750.000 cell mesh that extended 4 diameters from the rotor [123]. The author found that insufficient grid resolution created erroneous results above $10 \mathrm{~m} / \mathrm{s}$, where portions of the blade were fully stalled. A similar study by Duque in 1999 analysed the flow over the NREL Phase II 3-bladed rotor using the OVERFLOW computational code [124].

As part of the blind comparison test of the NREL Phase VI wind tunnel experiment. Sørensen et al. used the EllipSys3D CFD solver to perform steady and unsteady computations [125]. In these simulations, the tower and nacelle were neglected, a single rotor blade was modelled, and a uniform velocity field was prescribed. Over 3 million cells were used to model the rotor and freestream and simulations required 40.8 hours on 4 CPUs [125].

In 2007. Massouh et al. compared PIV measurements of a 3-bladed scaled wind turbine with RANS simulations using Fluent [126]. The CFD simulation used six million cells. a sliding mesh and did not apply rotational periodicity in order to perform yawed simulations. It was found that the CFD-predicted vortex core radii were smaller and the distance between vortex cores was greater than measured in the experiment. with weaker vortices that dissipated more rapidly than the experimental observations [126].

A more recent set of studies has demonstrated both the applicability of RANS CFD simulations to predict blade loads and their inability to resolve the far wake. A study conducted in 2009 by Fletcher et al. compared RANS and vorticity transport method 
(VTM) simulations of the NREL Phase VI turbine [120]. It was demonstrated that the RANS predictions of the normal and tangential force coefficients. as well as the chordwise pressure distributions. were more accurate than the VTM predictions. In this particular study, the wake structure predicted with the two methods was compared and it was demonstrated that the VTM preserved the wake vorticity for many revolutions downstream with minimal numerical diffusion whereas the wake in the RANS simulations dissipated after 1.5 rotor revolutions [120]. A similar study conducted by Bechmann et al. in 2009 presented RANS simulations of the experimental three-bladed MEXICO rotor and compared the predicted blade loads and the wake structure to experimental pressure measurements and PIV, respectively [127]. The EllipSys3D incompressible finite volume method CFD code from Risø was used with the $k-\omega$ SST turbulence model. A hyperbolic mesh topology consisting of 16 million nodes extended 7 diameters downstream of the blades. The CFD simulations properly characterized the axial induction in the wake up to 1.3 diameters downstream (the limit of the PIV measurements). but the author attempted to extend the measurement database using the simulation results. It was observed that a nonphysical acceleration in the wake occurs approximately 2 to 2.5 diameters downstream of the rotor due to numerical errors caused by the coarsening mesh [127].

RANS simulations were conducted by Réthoré et al. as part of the MEXNEXT program that followed the experimental wind tunnel campaign using the $4.5 \mathrm{~m}$ diameter MEXICO turbine [128]. These computations included the geometry of the open-jet wind tunnel nozzle. the downstream collector. as well as the room, and were designed to evaluate the effect of the wind tunnel on the development of the inflow and wake of the turbine. It was noted in Ref. [128] that the wind tunnel collector appeared to influence the wake $1.5 \mathrm{D}$ downstream of the rotor and that the nozzle could also affect the flow more than $1 D$ upstream of the rotor plane. The PIV experiments conducted as part of the measurement campaign with the MEXICO rotor were conducted within this range of values. 
The sophistication of rotating RANS CFD simulations has increased throughout the past decade. The initial results of the NREL blind comparison study demonstrated that earlier CFD computations of wind turbines typically had errors of $25-60 \%$ in the predicted power output [48]. Recent computational efforts have produced errors in the predicted blade loading of 5-20\% compared to experimental results [57.120.129]. RANS simulations have demonstrated an adequate capability of predicting blade loading and power output. but they are typically not well suited to accurately resolve the wake. The high numerical dissipation that is present in recent simulations and the coarsening mesh downstream of the blades typically causes the wake to artificially dissipate within 1 to 2 rotor diameters.

A more recent development has been the use of LES to resolve the wake downstream of a single rotor and wakes on a wind farm scale. The results of a LES computation were published in 2013 by Mo et al. in which the NREL Phase VI wind turbine was simulated inside a domain that mimicked the NASA Ames $24 \mathrm{~m} \times 36 \mathrm{~m}$ wind tunnel [130]. The simulations modelled the two rotor blades, the tower. and a simplified nacelle. Good agreement was observed between the experiment and the computation when considering the pressure distribution over the rotor blades and the turbine power output. The helical vortex generated by the turbine was investigated in detail in Ref. [130]. The helical tip vortices took on a sinuous shape approximately $4 \mathrm{D}$ downstream of the turbine and broke down 5D downstream. The authors suggested that the boundary between the near and far wake regions should correspond to the downstream distance where the tip vortices breakdown [130].

\subsubsection{Actuator Line Techniques}

In many cases where the wake is the primary region of interest. the rotor blades do not have to be modelled in a simulation. An alternative is to impose the effect of the blades on 
the simulation domain using an actuator disc or actuator line technique. Actuator methods represent a wind turbine rotor as a discontinuous disc or a set of lines within a CFD domain. The flow field is typically computed by a RANS or LES CFD solver and the aerodynamic forces that represent individual blades are imposed onto the computational grid [131.132] Actuator line methods have been applied to a wide range of problems. including yawed simulations [19], turbine wake interaction studies [133,134], and the effect of unsteady flow on a turbine [135]. Recently, Breton and Masson developed an actuator surface method to produce a more accurate representation of the rotor blades and validated the technique using the three-bladed $10 \mathrm{~m}$ NREL Phase III rotor [136]. It was demonstrated in Ref. [136] that the turbine power was well predicted at low speeds, but was over-predicted at high wind speeds due to the use of two-dimensional airfoil data on a stall-controlled rotor.

Actuator disc methods have also been used in tidal current turbines to evaluate the downstream wake development and the flow around the disc. Nishino and Willden used an actuator disc coupled to a RANS simulation that was designed to evaluate blockage and aspect ratio effects in a narrow channel [71]. In a similar manner, Turnock et al. applied a combined RANS-BEMT method in order to evaluate blockage effects on the velocity distribution of the flow bypassing the simulated rotor disc [72].

Actuator line methods were also coupled to the EllipSys3D CFD software by Sørensen $e t$ al. and Mikkelsen [135.137] and have recently been used for grid-based LES [138-140]. This actuator line-LES software has been used in a uniform inflow by Troldborg et al. to perform computations on a laterally-aligned column of three turbines separated by 3D [138] and to perform LES on a $3 \mathrm{MW}$ wind turbine in order to evaluate the effect of turbulence levels on the wake development downstream of a rotor [140]. Additional work by Mikkelsen et al. using the same software investigated the power output of three, longitudinally-aligned turbines with different blade pitch settings where the downstream turbines were either operating in full-wake or partial wake conditions [139]. Churchfield et al. used actuator 
lines coupled to a LES simulation in order to evaluate the effects of longitudinal and lateral spacing of tidal current turbines [141].

The aerodynamic forces in actuator line methods can be determined with two-dimensional BEMTs with corrections for tip losses and yawed flow and as such. can experience the limitations of blade element momentum theory when predicting blade loads [19]. A relatively small number of grid points are required in order to characterise the effect of the blades on the flowfield. however. compared to the large computational requirements for simulating the actual geometry of the blades and LES methods have been shown to accurately characterise wind turbine wake development $[130,140]$.

\subsubsection{Hybrid CFD-Vortex Methods}

Hybrid methods have also been developed recently as a way to model blade loads and the wake behind turbines using the combined benefits of RANS and vortex methods. A small region around the turbine blade is modelled with a computational mesh and the three-dimensional effects of the flow on the rotor blade are resolved with grid-based RANS. The vorticity in the flow is then carried from the RANS region using one of many available vortex-wake techniques. Hybrid CFD-Vortex methods have been developed by $\mathrm{Xu}$ and Benjanirat $[142,143]$ and Schmitz and Chattot $[103,129,144,145]$. Another solver developed by Anusonti-Inthra and Floros coupled a VPM with a RANS zone around a stationary wing [146]. This code was validated against an experiment performed by NASA on a NACA 0015 semi-span wing and demonstrated that the lack of significant numerical dissipation in the vortex particle method allowed the wake structure to be validated 6 chord lengths downstream of the wing. Recent work performed by Oxley at Carleton University introduced a novel compressible formulation for vortex particles that was coupled to the Carleton Multi-Block (CMB) Euler solver [147]. This unsteady code was validated against 
AGARD oscillating airfoil experiments and has demonstrated the capability to simulate airfoil-vortex interaction [147]. 


\section{Chapter 3}

\section{Experimental and Computational Methods}

A variety of experimental techniques were applied in order to characterise wind turbine performance. tip vortex development. and wind turbine wake profiles. This Section will describe the scaled rotor designs and the range of experimental and computational methods that were implemented.

\subsection{Scaled wind turbines}

The water channel and wind tunnel experiments conducted in this thesis used two different rotor designs. The first design was a three-bladed wind turbine designed in the Department of Mechanical and Aerospace Engineering (MAAE) at Carleton and the second rotor was a geometrically-scaled version of an existing. medium-scale rotor. 


\subsubsection{MAAE rotor}

A three-bladed, fixed-pitch, wind turbine rotor has been designed for wind turbine experiments. A solid model of the scaled wind turbine and a generator can be seen in Fig. 3.1. The rotor blades were manufactured with an Objet Poly Jet three-dimensional printer and the blades were printed using a layer thickness of $16 \mu \mathrm{m}$. The diameters of the printed rotors ranged from $20 \mathrm{~cm}$ to $40 \mathrm{~cm}$. A $40 \mathrm{~cm}$ rotor was the largest size that could be printed with the PolyJet printer and a $15 \mathrm{~cm}$ rotor was printed but did not generate enough torque to reach the desired operating conditions.

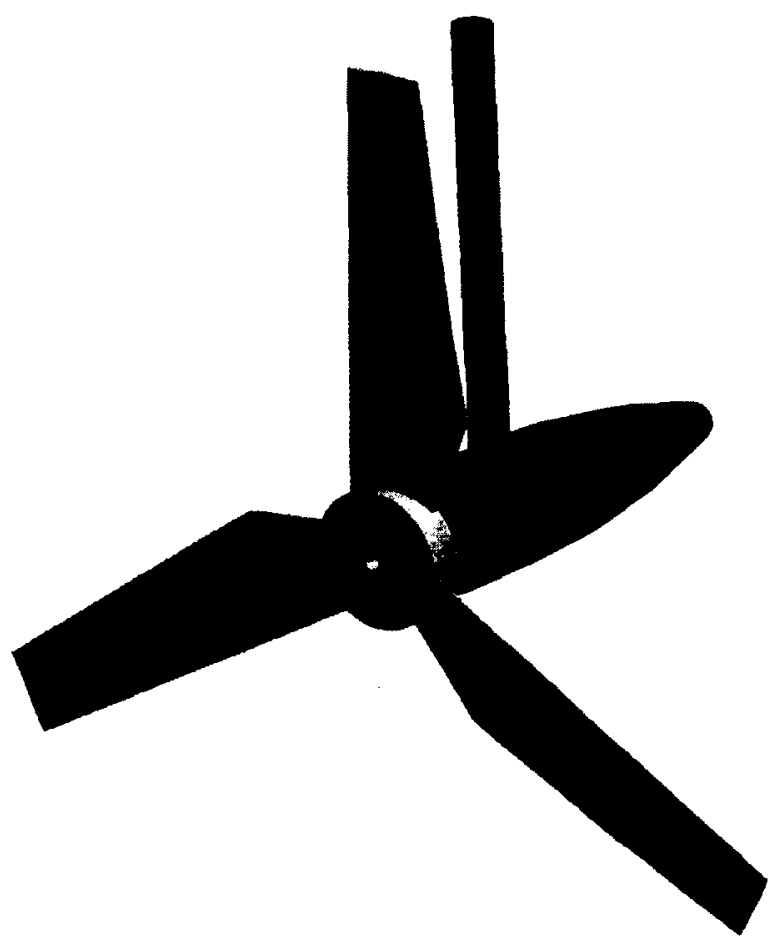

Figure 3.1: Solid model of the scaled MAAE wind turbine.

Several scaling relationships had to be considered during the design of the scaled rotors and these factors relate to the Reynolds number. Mach number, rotor geometry. and dynamic motion. Many aerodynamic phenomena. including separation and vortex shedding. 
are dependent on the Reynolds number of the flow. The Reynolds number based on the tip conditions is defined in Equation (3.1), where $l_{t i p}$ and $c_{t i p}$ are the tip speed and the blade tip chord. respectively. and $\nu$ is the kinematic viscosity of the fluid. The Reynolds number based on the blade tip speed and tip chord will be referred to simply as the Reynolds number in the remainder of the thesis.

$$
R e_{t i p}=\frac{U_{t i p} c_{t i p}}{\nu}
$$

Reynolds similarity is typically not maintained between commercial-scale turbines and small-scale models and the reasoning behind this can be illustrated by the work conducted by Burdett and Van Treuren [32]. Scaling relationships were developed and identify the scaling factor $\left(F_{s}\right)$ required to maintain Reynolds similarity between a commercial-scale turbine with radius $R_{c}$ and a model with radius $R_{m}$ as described in Equation (3.2) [32].

$$
F_{s}=\frac{R_{c}}{R_{r n}}
$$

The rotational speed of the model $\left(\Omega_{m}\right)$ required to maintain Reynolds similarity with the commercial-scale rotor whose rotational speed is $\Omega_{c}$ can then be described as a function of $F_{s}$ by Equation (3.3) [32].

$$
\Omega_{m}=\Omega_{c} F_{s}^{2}
$$

The current scaled rotors would have a scaling factor $F_{s}$ of approximately 500 for a 20 $\mathrm{cm}$ model based on a $100 \mathrm{~m}$ commercial wind turbine. This would require an extremely large rotational speed to maintain the Reynolds number which would violate Mach number 
similarity and result in aerodynamic phenomena. such as shock waves, not seen on wind turbine blades. It is therefore more desirable to maintain Mach similarity between models than it is to maintain Reynolds number similarity. As a result. the Nach number based on the blade tip speed was maintained at below 0.2 in the experiments conducted in air for similarity with blade tip Mach numbers observed on commercial-scale turbines. The Mach number based on the blade tip speed effectively limited the Reynolds number based on the blade tip conditions to values on the order of $10^{4}$.

The three-bladed MAAE rotor was not intended to act as a scaled version of a commercial-scale rotor, and therefore geometric similarity did not need to be maintained with this model. As will be discussed in greater detail below, the normalised chord of the rotor blades was increased relative to commercial-scale turbines in order to maximise the Reynolds numbers that could be reached by the turbine while respecting the Mach scaling limitation on the tip speed.

Dynamic similarity, however, can be maintained between models and commercial-scale rotors and the parameter that can be matched to this effect is the tip speed ratio $(\lambda)$. The tip speed ratio represents the blade tip speed divided by the freestream speed. Commercialscale wind turbines typically have a tip speed ratio of 6 to 8 at mean annual wind speeds and properties such as the turbine thrust and wake expansion have been shown to be related to the tip speed ratio [40]. The current rotor was therefore designed to operate in this tip speed ratio regime.

The wind turbine was also designed to generate a thrust coefficient representative of that produced by larger. commercial-scale designs. The thrust coefficient is related to the momentum deficit in the wake, the wake expansion, and the downstream speed recovery. The applicability of the results from scaled experiments to commercial-scale scenarios will therefore depend in part on mimicking large-scale thrust coefficients. The power coefficient 
of the turbine was not an important consideration with the MAAE rotor as scaled turbines have power coefficients which are much lower than commercial-scale designs [79].

The current rotors were operated at Reynolds numbers that ranged from 3,620 to 47.000. based on the blade tip speed and tip chord. There is a limited amount of experimental lift curve data available for airfoils at Reynolds numbers between 10.000 and 100.000 . as this is a range of Reynolds numbers that falls between the Reynolds numbers typically seen on Micro Air Vehicles (MAV) and Uninhabited Air Vehicles (UAV). The lift. drag. and stall behaviour of an airfoil can become highly sensitive to changes in operating conditions at Reynolds numbers on the order of $10^{4}$ and thick airfoils typically do not perform well in this flow regime [148]. As an alternative, an Eppler E387 airfoil was selected for the rotor blades due to the airfoil's lift curve behaviour and the availability of experimental data near the expected Reynolds number regime [149-151]. The E387 airfoil was designed principally for low-speed applications where the Reynolds number is below 500,000 and has a thicknessto-chord ratio that is one half the thickness of contemporary wind turbine airfoils. The E387 airfoil has a camber of $3.7 \%$ and a thickness-to-chord ratio of $9 \%$ and its geometric profile is shown in Figure 3.2(a). By comparison. modern wind turbine airfoils have been designed for commercial-scale conditions and can have a thickness-to-chord ratio that is approximately $20-25 \%$.

The selection of a thin airfoil for the MAAE was predominantly a byproduct of the desire to use an airfoil that exhibited reasonable and quantifiable lift properties at the Reynolds numbers under consideration in these experiments. Work by Burdett and Van Treuren has indicated that thick, wind-turbine specific airfoils can also be used on scaled models [32]. The experiments presented in Ref. [32] generated airfoil data for a $21 \%$ thick NREL S823 airfoil at Reynolds numbers that ranged from 50,000 to 275.000 . The airfoils did not suffer from degraded performance due to laminar separation at low Reynolds numbers provided that a trip strip was integrated on the scaled models. The geometry of the S823 airfoil is 


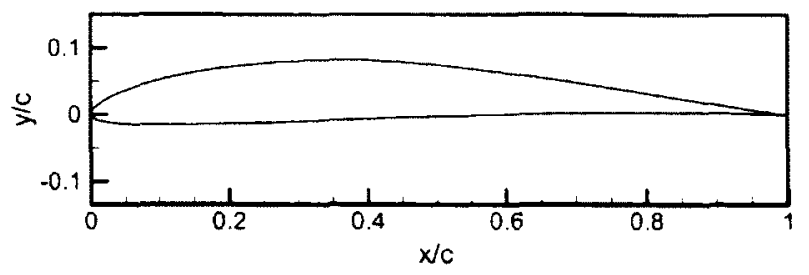

(a) E387 airfoil.

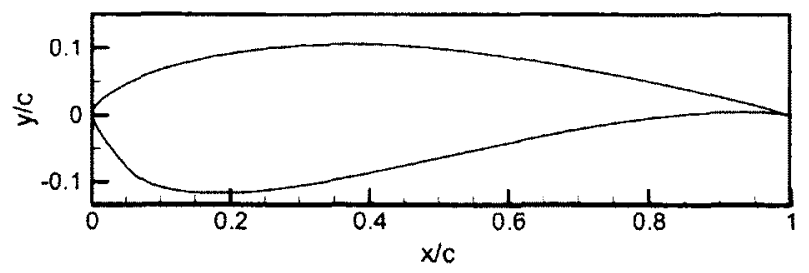

(b) $\mathrm{S} 823$ airfoil.

Figure 3.2: Comparison of the E387 airfoil with a typical wind turbine airfoil.

compared to the E387 airfoil in Figure 3.2.

Experimental lift data for the E387 airfoil from Refs. [149-151] is presented in Figure 3.3 for Reynolds numbers ranging from 10,000 to 50.000 . The airfoil has a shallow lift curve at Reynolds numbers below 30.000 and has a relatively low lift to drag ratio of $7-9$ when the Reynolds number ranges from 10.000-20.000. The airfoil also exhibits a characteristic jump in lift at $\alpha=10^{\circ}$ when $R e=30.000$ and a similar increase at $\alpha=8^{\circ}$ when $R e=40.000$. This behaviour was evaluated by McArthur and was attributed to the formation of a laminar separation bubble at these particular flow conditions followed by subsequent transition to turbulence and flow reattachment, which results in the increase in lift [151].

Once the experimental lift and drag data had been obtained for the E387 airfoil. the geometry of the blades could be determined. The chord $(c)$ and twist $(\beta)$ distributions of the rotor blades were developed using blade element momentum theory from Ref. [86] and are presented in Figures 3.4(a) and 3.4(b). respectively. Blade element momentum theory (BEMT) combines momentum theory and blade element theory in order to resolve the performance of a turbine design. Two-dimensional airfoil data is required in a BEMT method to calculate the tangential and thrust forces generated by a series of elements along 


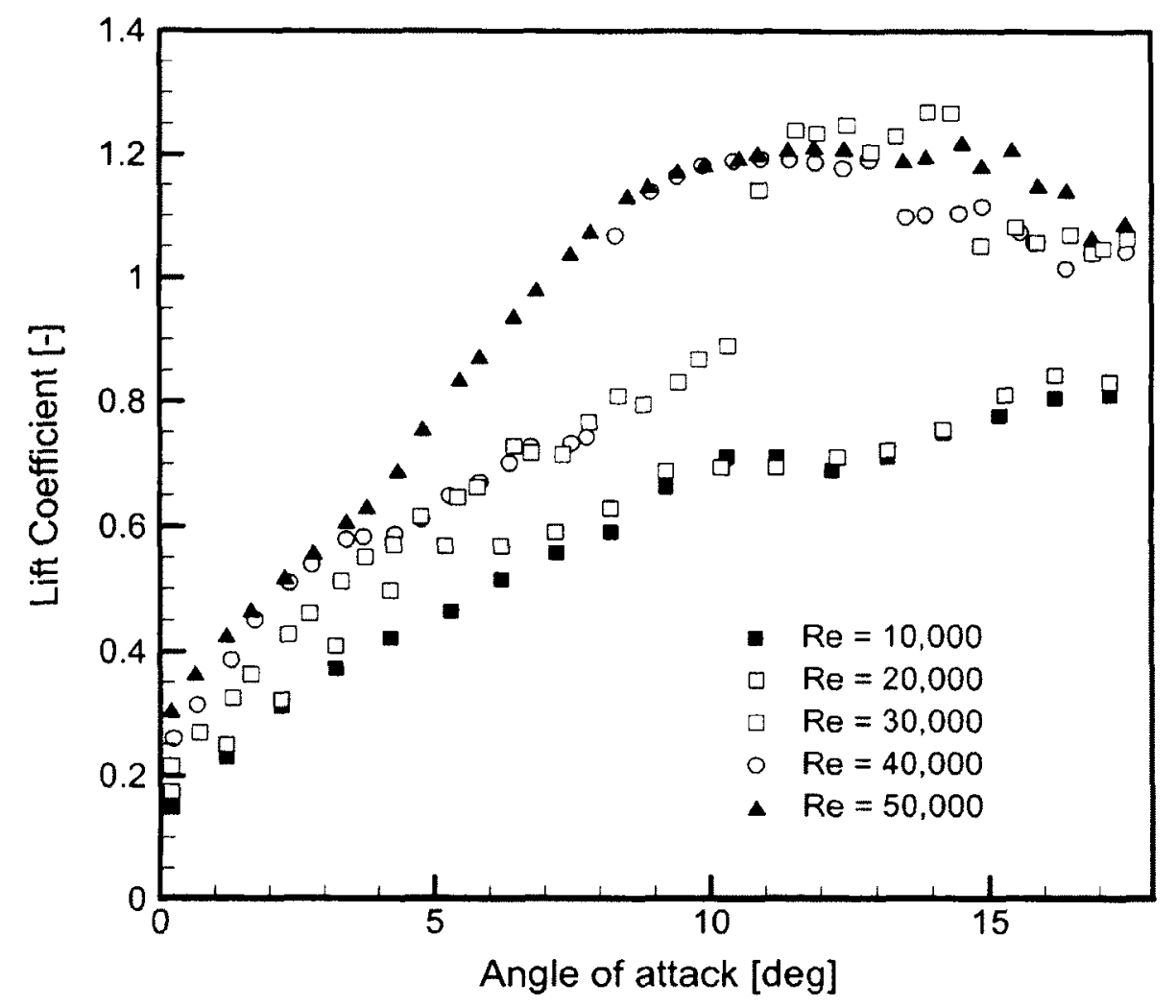

Figure 3.3: Eppler E387 lift curves at low Reynolds numbers [149-151].

the rotor blade and the axial and radial induction factors are determined iteratively at a particular $\lambda$. The background theory for BEMT is well described in literature and will not be repeated here [86].

The normalized chord of the scaled blades was increased relative to that observed on fullscale designs in order to increase the Reynolds number of the blades. The twist distribution of the blades was modified from an ideal twist distribution determined with BEMT due to the desire to produce angles of attack in the unstalled region of the E387 lift curve with tip speed ratios from 4 to 8 in the expected Reynolds number regime.

As a consequence of increasing the magnitude of the Reynolds numbers that could be attained by the rotor blades. the solidity of the rotor blades will be larger than that observed on commercial wind turbines. The solidity of the rotor blades can be determined 


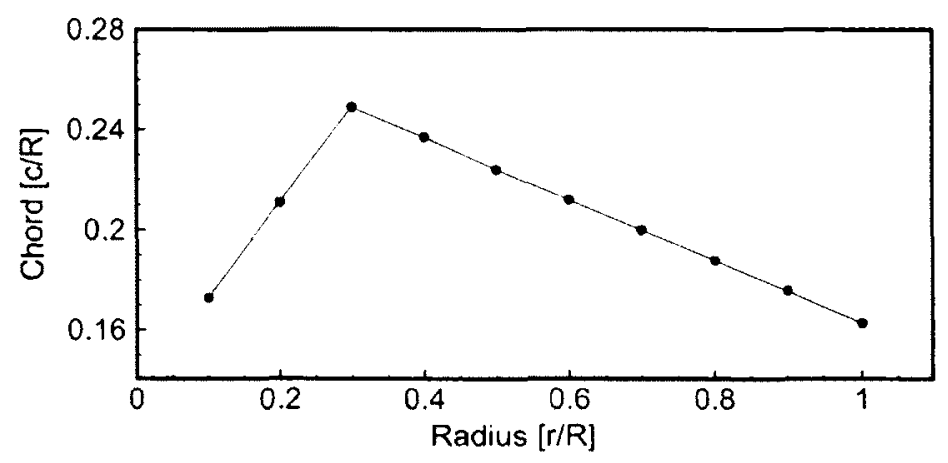

(a) Chord distribution.

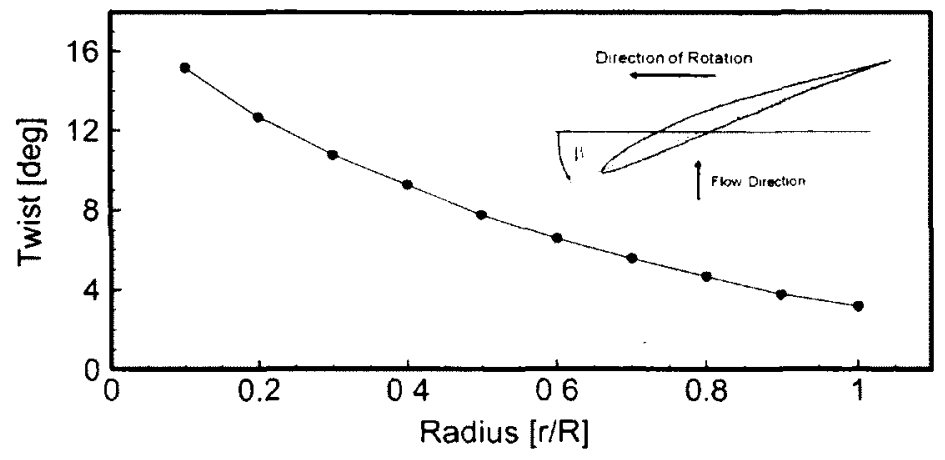

(b) Twist distribution.

Figure 3.4: The chord and twist distribution of the scaled MAAE rotor blades.

using Equation (3.4) and represents the ratio between the frontal area of the blades $\left(A_{\text {blades }}\right)$ and the swept area of the rotor $\left(A_{\text {swept }}\right)$. The solidity of the current design is $18 \%$ and is 3 to 4 times higher than the solidity of typical. in-service rotors [9]. The high rotor solidity resulting from the chord design choice will result in a narrow power coefficient curve.

$$
\text { Solidity }=\frac{A_{\text {blades }}}{A_{\text {swept }}}
$$

The scaling parameters and geometric design parameters for the MAAE wind turbine blades are summarized and are compared to typical commercial-scale values in Table 3.1. 
Table 3.1: MAAE Design Parameters.

\begin{tabular}{|c|c|c|}
\hline Parameter & MAAE Rotor & Typical Full-Scale Values [9] \\
\hline \hline Diameter & $20-40 \mathrm{~cm}$ & $80-100 \mathrm{~m}$ \\
\hline Tip chord, c/R & 0.163 & $0.01(1 \mathrm{~m})$ \\
\hline Tip speed ratios & $6-8$ & $6-10$ \\
\hline Tip Reynolds number & $3,600-47.000$ & $1 \times 10^{6}-1 \times 10^{7}$ \\
\hline Tip Mach number & $0.05-0.18$ & $0.2-0.25$ \\
\hline Thrust coefficient & $0.8-0.9$ & $0.8-0.9$ \\
\hline Solidity & $18 \%$ & $4 \%$ to $5 \%$ \\
\hline Airfoil & Eppler E387 & Various \\
\hline Airfoil thickness to chord & $9 \%$ & $20 \%-25 \%$ \\
\hline
\end{tabular}

\subsubsection{Scaled TU Delft turbine}

An experiment was conducted in 2005 by Haans et al. at TU Delft using a two-bladed rotor in an open-jet wind tunnel in order to determine the wake expansion in axial and yawed flow using smoke visualisation. The TU Delft rotor had a NACA 0012 airfoil and a diameter of $1.2 \mathrm{~m} \mathrm{[40].} \mathrm{The} \mathrm{rotor} \mathrm{had} \mathrm{a} \mathrm{linear} \mathrm{twist} \mathrm{distribution} \mathrm{from} \mathrm{the} \mathrm{root} \mathrm{cutout} \mathrm{to} \mathrm{the}$ $90 \%$ radial location. followed by a constant twist to the blade tip. The tip pitch angle of the rotor could be set at $0^{\circ}, 2^{\circ}$. or $4^{\circ}$. The rotor was tested in uniform flow at $5.5 \mathrm{~m} / \mathrm{s}$ and at tip speed ratios that ranged from 6 to 10. These conditions corresponded to a Reynolds number range of 175.000-291.000, based on the blade tip speed and tip chord [40].

In order to gain a deeper understanding of how scaled wind turbine experiments reflect medium- or full-scale conditions, a geometrically-scaled version of the TU Delft rotor was created to support this thesis research. The non-dimensionalised chord and twist distributions from the medium-scale rotor were therefore maintained in the scaled model. The $1 / 4.8$ scaled rotor had a diameter of $25 \mathrm{~cm}$ and was tested with Reynolds numbers on the order of $10^{4}$. similar to the MAAE rotor. A solid model of the $25 \mathrm{~cm}$ diameter scaled TU Delft rotor with $\theta_{t i p}=0^{\circ}$ is shown in Figure 3.5. The pitch angle of the rotor blades in the medium-scale experiment could be changed manually. Given the small size of the $1 / 4.8$ 
scale rotor. a more convenient solution was to print two different models with the desired tip pitch angles. As a result. two scaled TU Delft rotors were manufactured using the same method as used for the MAAE rotor with tip pitch angles of $0^{\circ}$ and $4^{\circ}$.

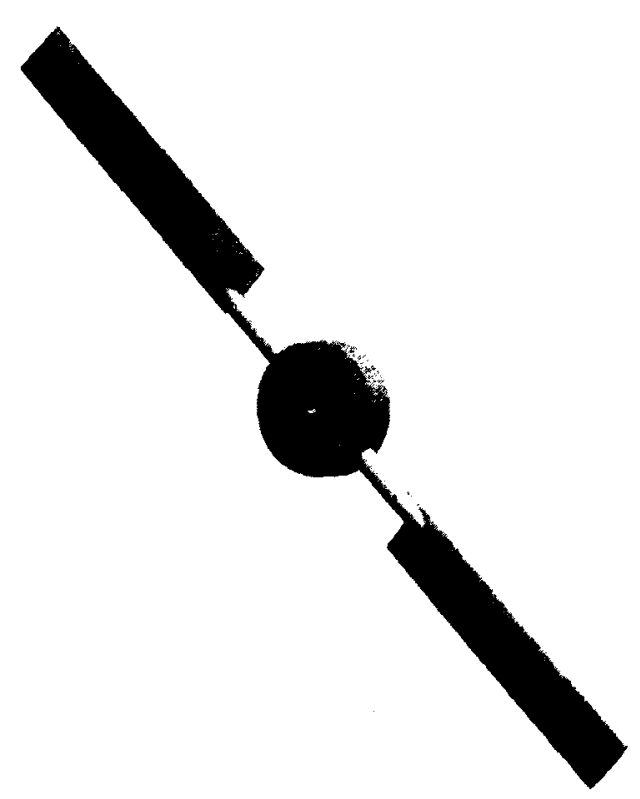

Figure 3.5: Solid model of the scaled TUDelft rotor.

A comparison between the small-scale rotor used in the current study and the mediumscale experiment presented by Haans et al. is provided in Table 3.2 [40].

\subsection{Water channel experiments}

The experiments that were conducted to identify blockage effects and Reynolds number effects in scaled wind turbine testing took place in the water channel facility at Carleton University. The water channel at Carleton was manufactured by Rolling Hills Research Corporation (RHRC) and was commissioned in 2008 [152]. The commercially-available 
Table 3.2: Comparison between the full-scale TU Delft rotor and the $1 / 4.8$ scale rotor.

\begin{tabular}{|c|c|c|}
\hline Parameter & TU Delft & Carleton \\
\hline \hline Blades & 2 & 2 \\
\hline Airfoil & NACA 0012 & NACA 0012 \\
\hline Diameter & $1.2 \mathrm{~m}$ & $0.25 \mathrm{~m}$ \\
\hline Radius & $0.6 \mathrm{~m}$ & $0.125 \mathrm{~m}$ \\
\hline Root cutout & $0.3 \mathrm{R}$ & $0.3 \mathrm{R}$ \\
\hline Chord & $0.08 \mathrm{~m}$ & $0.01667 \mathrm{~m}$ \\
\hline$\lambda$ & $6,8.10$ & 6.8 \\
\hline$U_{\infty}$ & $5.5 \mathrm{~m} / \mathrm{s}($ Air $)$ & $0.028 \mathrm{~m} / \mathrm{s}-0.23 \mathrm{~m} / \mathrm{s}\left(\mathrm{H}_{2} O\right)$ \\
\hline$R e_{t i p}$ & $175,000(\lambda=6)$ & $12,800-29,100(\lambda=6)$ \\
& $230,000(\lambda=8)$ & $28,200-31,400(\lambda=8)$ \\
\hline$\theta_{\text {tip }}$ & $0^{\circ}, 2^{\circ}, 4^{\circ}$ & $0^{\circ}, 4^{\circ}$ \\
\hline Twist & $\left(6+\theta_{t i p}\right)-6.67 r / R,-0.3 \leq r / R \leq 0.9$ & same as full-scale \\
& $\theta_{t i p}, 0.9<r / R \leq 1$ & \\
\hline
\end{tabular}

RHRC 2436 closed-loop water channel shown in Figure 3.6 has a $1.82 \mathrm{~m}$ long test section with a $0.61 \mathrm{~m} \times 0.81 \mathrm{~m}$ cross-section. The water channel can be operated at flow speeds ranging from $0.025 \mathrm{~m} / \mathrm{s}$ to $0.31 \mathrm{~m} / \mathrm{s}$ with a turbulence intensity of $1 \%$ RMS [152]. The upstream flow conditioning elements consist of a series of screens and honeycomb and the walls then follow a 6:1 contraction ratio. Divergent glass walls compensate for boundary layer growth in the test section. The speed in the test section is inferred using a Signet 2536 Rotor-X Flow Sensor coupled to a Signet 8152 Flow Totalizer. The rotary flowmeter is located in the downstream return section of the water channel. A portion of the flow is diverted from the main return and passes through the flow meter. The speed output for Carleton's tunnel was calibrated by the manufacturer prior to installation of the water channel and requires the quiescent water height to be approximately $81 \mathrm{~cm}$. As a result. the water level was verified every day throughout the measurement campaign and water was added if necessary.

A pressurized. six-colour. dye delivery system integrated with the water channel uses food colouring diluted with water for flow visualisation. The dye system is composed of six canisters filled with dye arranged as shown in Figure 3.7. The canisters are pressurised to 


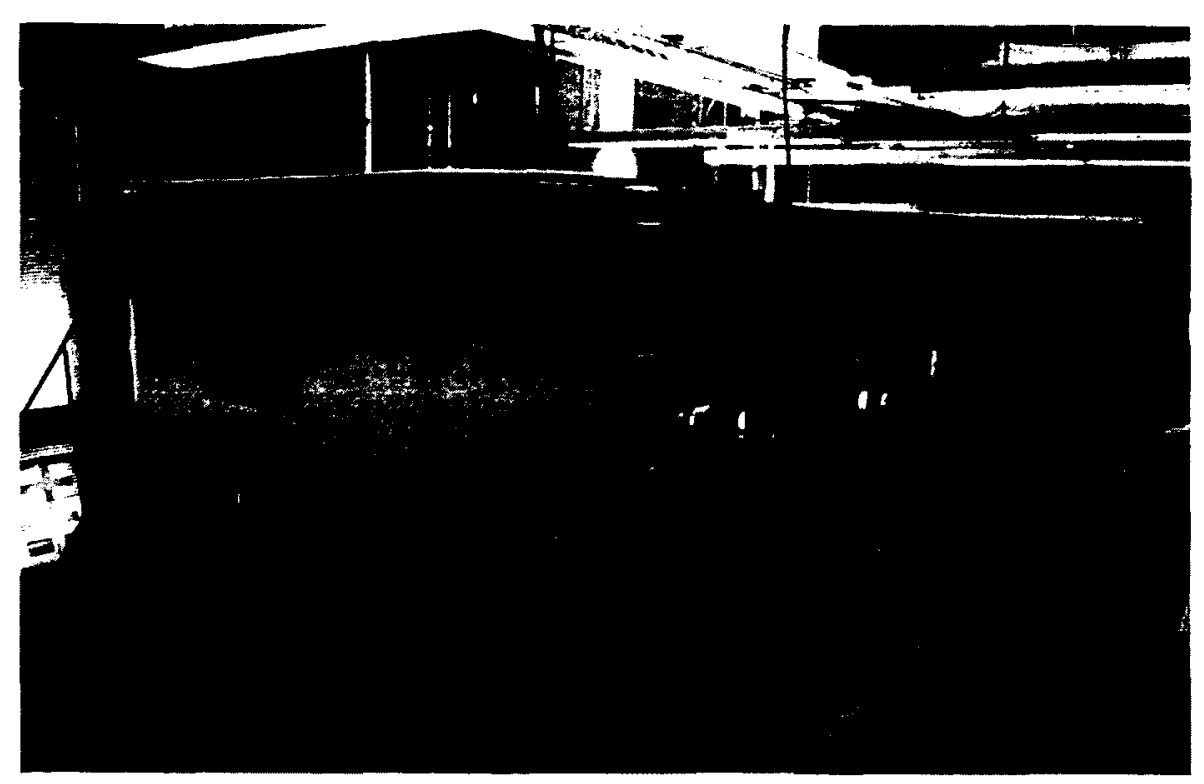

Figure 3.6: The RHRC 2436 water channel.

20 psi using regulated shop air and the flow rate of the dye from each canister is controlled independently with a control valve. The dye is delivered to the water channel in Tygon tubing and is injected into the flow in a tapered copper tube.

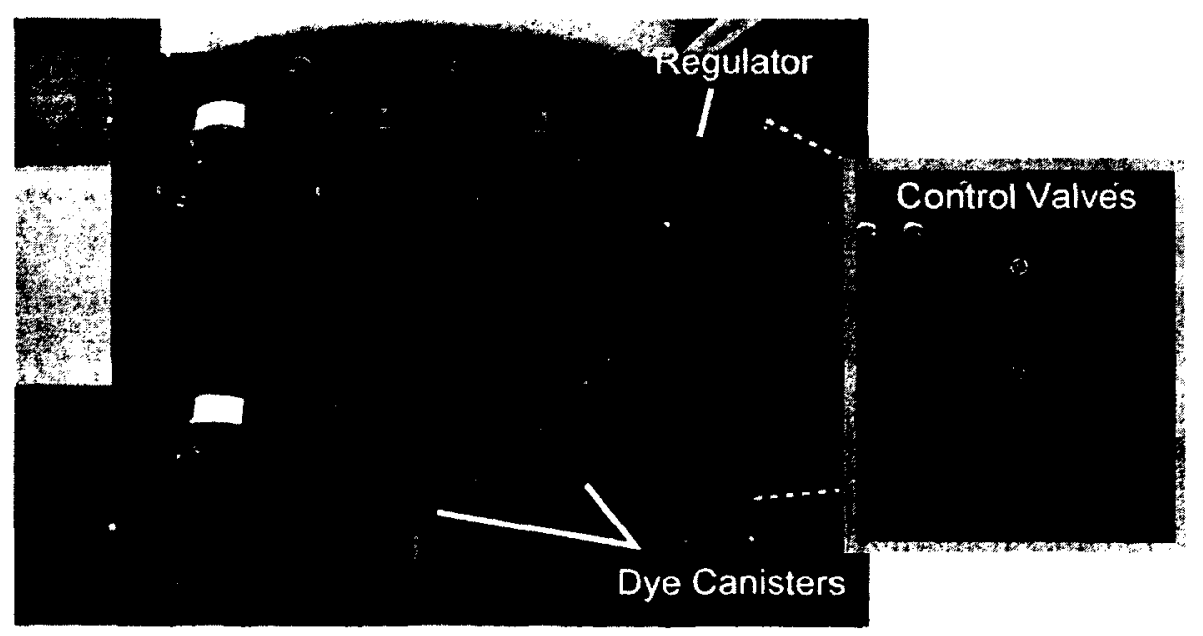

Figure 3.7: The pressurised dye delivery system.

When conducting the flow visualisation experiments. the wind turbine and the dye rig were mounted in the channel test section as shown in Figures $3.8(\mathrm{a})$ and $3.8(\mathrm{~b})$. The swept area of the rotor. the minimum tip clearance from the wall $(s)$. and the dye injection location 
are all indicated in Fig. 3.8(b). The dye was injected into the flow at the same height as the rotor axis near the blade tip. The dye strut was a streamlined rod and the tip of the dye injection tube was located sufficiently downstream of the dye strut to limit the effect of the strut wake on the injected dye stream. The rotor was mounted at the center of the water channel and the free surface was assumed to have a negligible influence on the recorded values, based on experiments in literature [30].

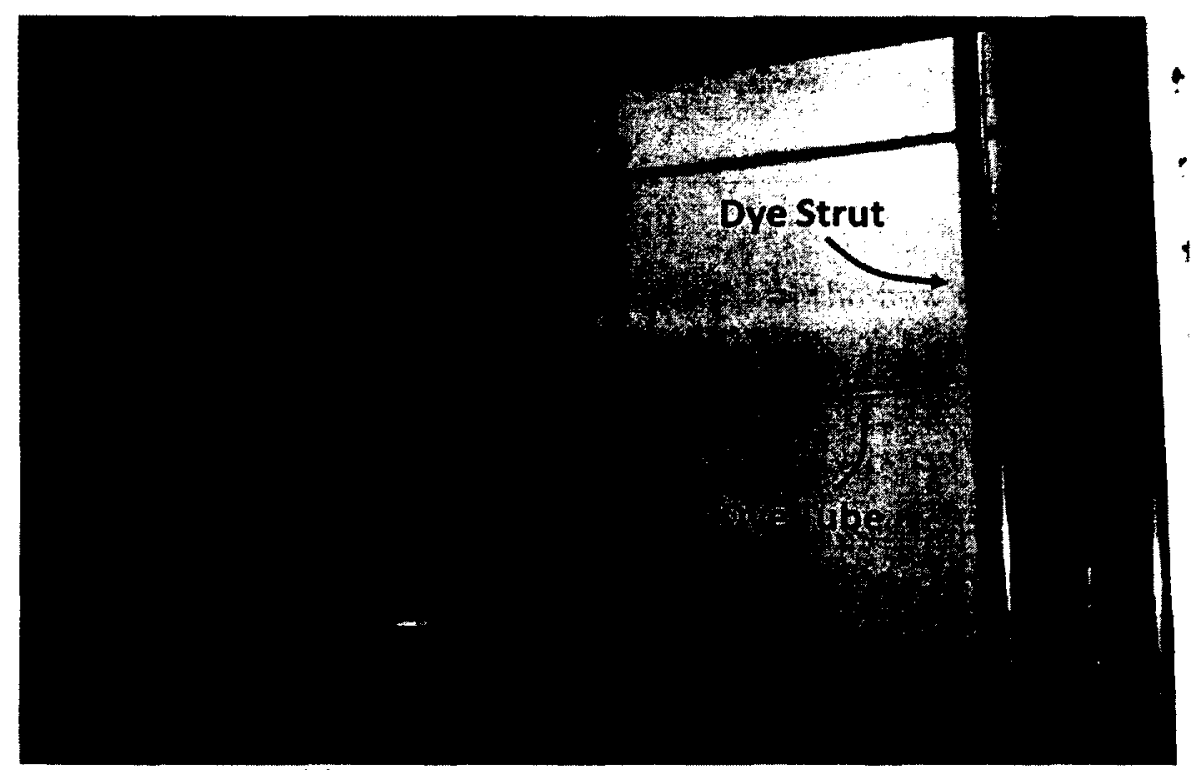

(a) Wind turbine and dye rig installation.

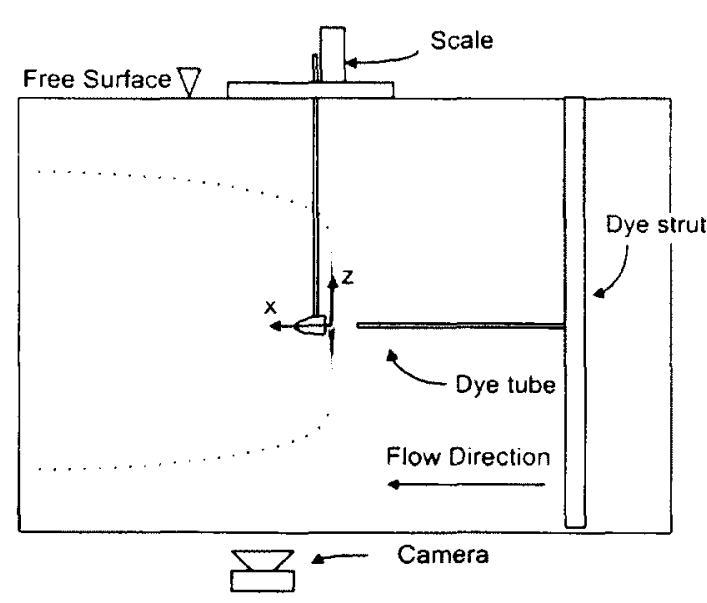

Side View of the Test Section

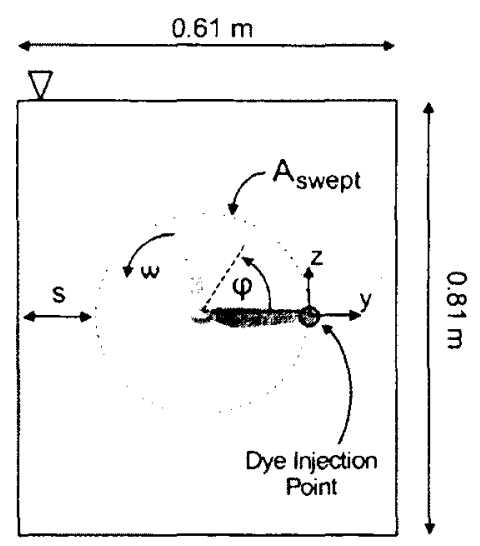

Front View

(struts omitted for clarity)

(b) Schematic of the experimental setup. The dimensions are not to scale

Figure 3.8: The RHRC 2436 water channel at Carleton Lniversity. 


\subsubsection{Quantitative dye visualisation and tip vortex identification}

Flow visualisation is frequently used as a qualitative technique that can highlight important flow features [153]. In some cases. however, quantitative information can be extracted from flow visualisation images. Haans et al. used smoke visualisation in air to identify tip vortex core coordinates downstream of a two-bladed horizontal axis wind turbine [40]. Aurahs et al. used a similar technique in water to evaluate the interaction of a horseshoe vortex with the rotor in an axial turbine [154]. Quantitative flow visualisation was used in the water channel in order to identify discrete tip vortex cores lying along a plane.

Photographs of the flow structures were recorded from below the glass floor of the test section using a Nikon D3100 digital SLR camera equipped with a AF-S NIKKOR $18-55 \mathrm{~mm}$ lens. The field of view was illuminated using two $500 \mathrm{~W}$ halogen lights. The relative locations of the tip vortex cores were identified using a $0.635 \mathrm{~cm} \times 0.635 \mathrm{~cm}$ grid located above the surface of the water. represented schematically in Figure 3.9. Images were recorded to capture both the streaklines and the measurement grid when the blade tip passing through the dye stream was in the $0^{\circ}$ azimuth position indicated earlier in Figure 3.8(b). The images were post-processed using the image analysis software Engauge and the tip vortex locations were identified with respect to the measurement grid visible in the photographs. It has been assumed that the centre of the tip vortex core corresponds to the tip vortex core location. following the techniques described in Ref. [40]. The twodimensional coordinates corresponding to the downstream $(x)$ and lateral $(y)$ position of the vortex cores relative to the blade tip were identified and were extracted from the image processing software. As a result. the coordinate system used to assess the expansion in the wake has its origin at the blade tip, as highlighted in Figures 3.8(b) and 3.9.

The identified vortex core coordinates relative to the grid have to be subsequently 


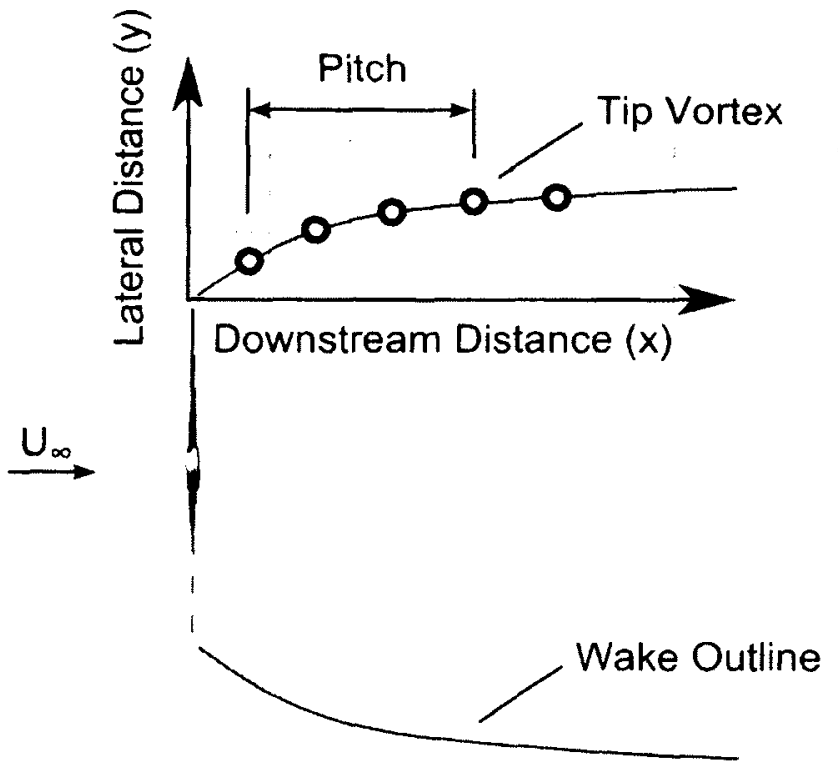

Figure 3.9: Schematic of the wake expansion and the tip vortex pitch.

corrected to represent the distances at the dye injection plane. This involves correcting the distances for camera parallax effects using the distance between the camera lens and the measurement grid $(H)$ and the distance between the measurement plane and the grid (h) following the procedure described in Ref. [40]. A schematic that identifies the distances used for parallax correction can be seen in Figure 3.10. The distance $L$ represents a distance identified based on the grid coordinates and $d$ represents the corresponding actual distance at the measurement plane. The photographed vortex cores are corrected for the effect of parallax using Equation (3.5).

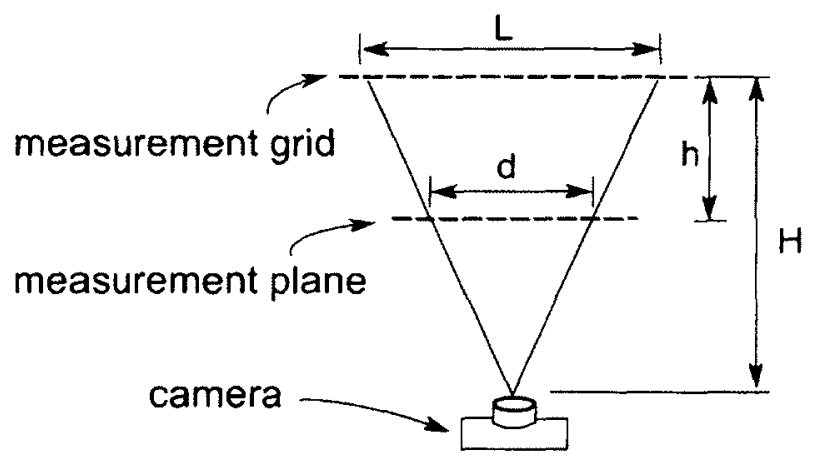

Figure 3.10: Parallax correction schematic. Adapted from [40]. 


$$
d=\frac{H-h}{H} L
$$

The downstream vortex core coordinates have been normalised by the turbine diameter $(x / D)$. The lateral positions of the tip vortex cores from the blade tip were normalised with respect to the diameter $(y / D)$ for consistency with the downstream coordinates. The initial wake expansion is characterised in this thesis by the lateral position of the tip vortex cores in the near wake region. The core locations determined from six images were averaged for consistency with the procedure outlined in Ref. [40]. Additional analysis confirmed that an acceptable level of repeatability was obtained when 5 or 6 images were used when calculating the average core locations. Additionally. a synchronisation mechanism was not used to record the photographs and the images were selected based on the resulting blade tip position. The errors in the mean vortex core location resulting from the experimental approach will be described in Chapter 4.

\subsubsection{Rotor control}

A set of GWS-400 generators and EPS-series gearboxes manufactured by Grand Wing Servo-Tech Co., Ltd were used to control the rotational speed of the rotors. The gearboxes were designed to slow the rotor down to the desired rotational speed and the selected gearbox depended on the rotor size and the flow speed. The reduction ratios of the 9 gearboxes ranged from 2.14 to 6.30 . Fine speed adjustments at a given flow speed were performed by adjusting the load on the generator using a resistor. 


\subsubsection{Scale measurements}

In addition to conducting flow visualisation for each test case. the thrust force generated by the rotor was inferred using a commercially-available AMTI HE6X6 six degree-offreedom balance. The HE6X6 force balance uses Hall Effect sensors to record displacements and calculates three forces and three moments. An image of the scale and the associated components can be seen in Figure 3.11. The balance measures $15.24 \mathrm{~cm} \times 15.24 \mathrm{~cm}(6$ in $\times 6$ in) and the balance platform has four mounting screws. Four additional holes are provided on the base of the scale to fix the balance within an experimental setup. The scale outputs digital data that is transmitted to a computer and to the AMTI NetForce software through a USB cable.

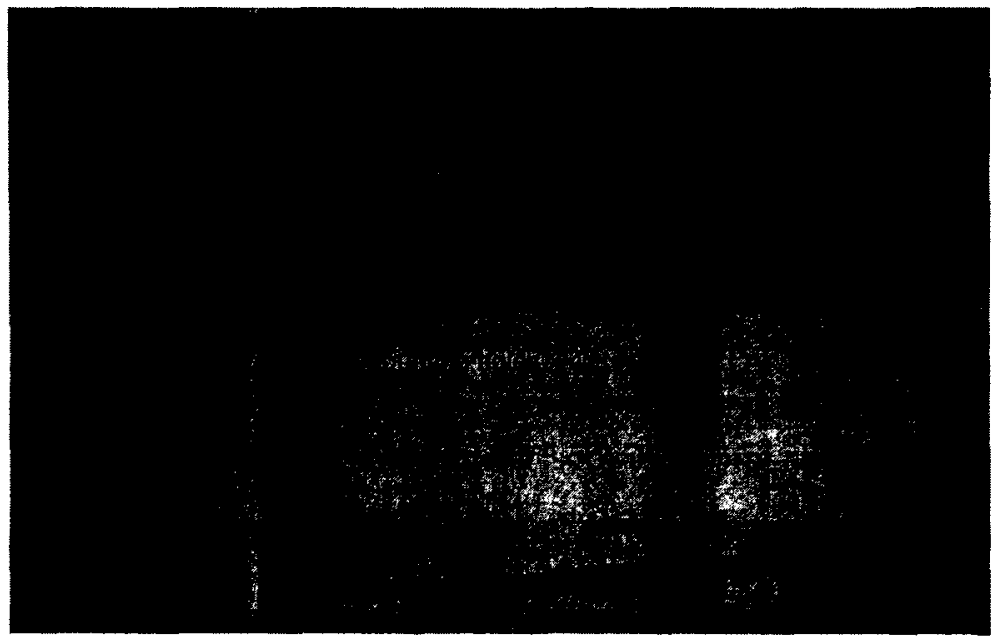

Figure 3.11: The HE6X6 scale and components.

A schematic of the top view of the balance indicating its coordinate system is shown in Figure 3.12. The five positions indicated on the right side of Figure 3.12 refer to the five locations where the balance was loaded during the calibration process that will be described below.

The six degree-of-freedom balance was predominantly used in the water channel experiments, where it was mounted in the vertical arrangement highlighted in Figure 3.13 . 

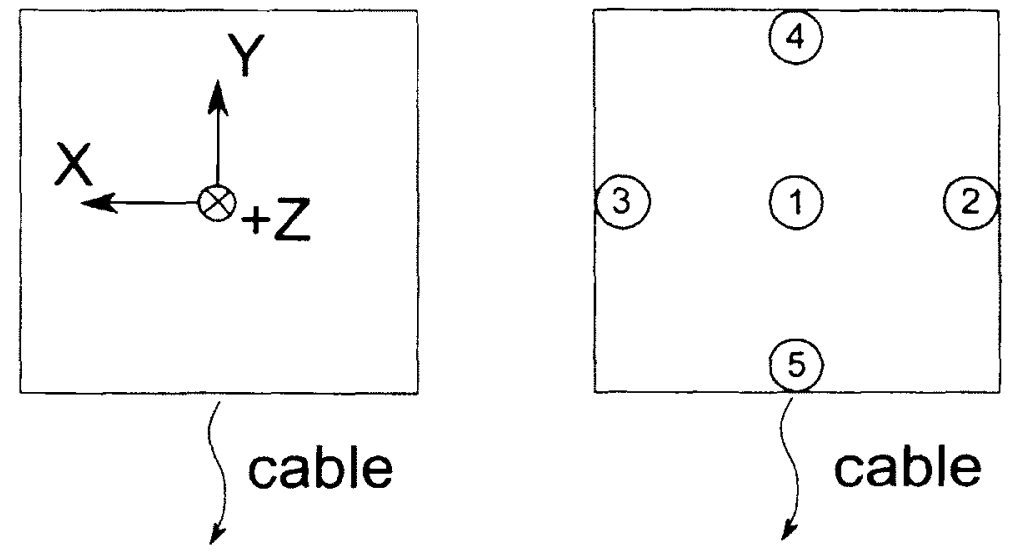

Figure 3.12: Schematic of the scale coordinates - top view.

The balance was attached to a mounting plate at a $90^{\circ}$ angle and a joining plate was used to connect the turbine support strut to the platform of the balance. In order to isolate the rotor-generated forces from the effect of the mean flow. a concentric pipe was place around the turbine support strut and was attached under the platform of the entire assembly.

The scale was also used for a small number of experiments in the upstream section of the wind tunnel. While the configuration of those experiments will be discussed in the next Section, the scale was arranged horizontally underneath the floor of the wind tunnel.
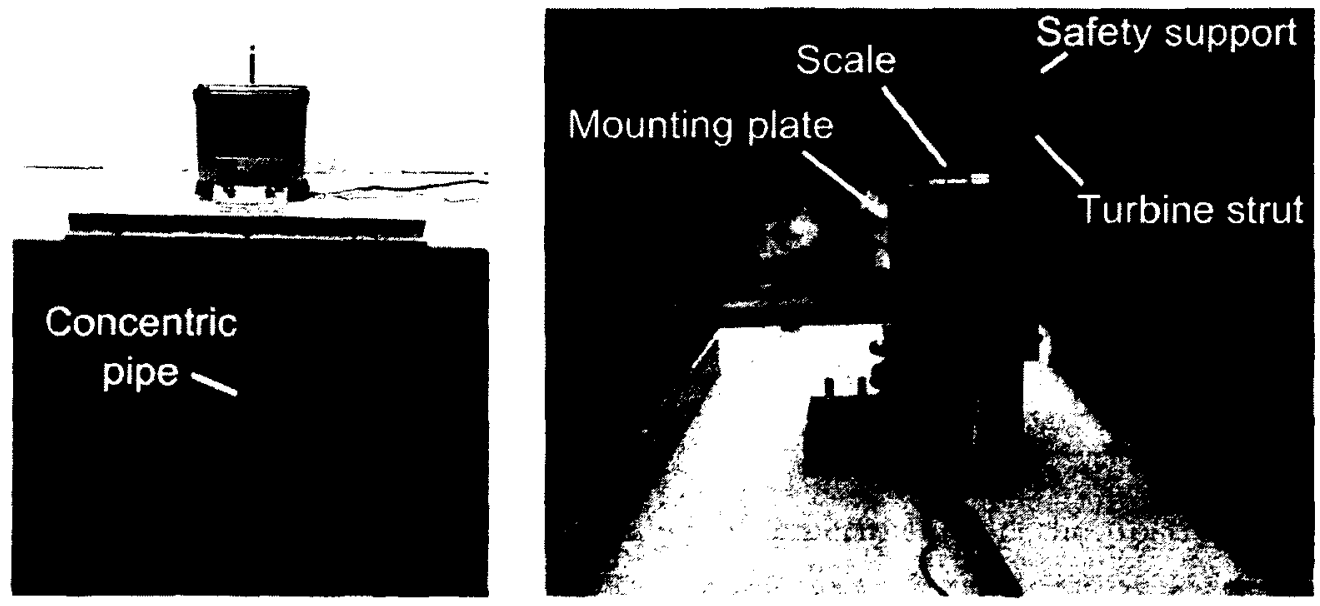

Figure 3.13: Vertical scale arrangement for the water channel experiments.

The balance was used to determine the thrust force generated by the wind turbines. as 
$C_{T}$ was related to the downstream velocity deficit and wake expansion. The thrust force generated by a turbine was obtained using the moment about the $\mathrm{x}$-axis $\left(\Lambda_{x}\right)$. instead of using the forces determined by the scale. In the vertical configuration, the direct thrust force corresponded to $F_{z}$ from the balance and the $F_{z}$ data output by the balance were not reliable in this orientation. The moment about the $\mathrm{x}$-axis and the distance between the rotor's centre of rotation and the centre of the balance were used to determine the thrust force $\left(F_{\text {Thrust }}\right)$. This approach was much more repeatable than using the $\operatorname{direct} F_{z}$ output from the balance. Once the thrust force had been calculated. the thrust coefficient $\left(C_{T}\right)$ was determined using Equation (3.6).

$$
C_{T}=\frac{F_{\text {Thrust }}}{0.5 \rho A_{\text {swept }} U_{\infty}^{2}}
$$

\subsubsection{Balance calibrations}

The balance was calibrated by the manufacturer and the calibration constants are applied in the AMTI NetForce software. The accuracy of the calibration curves for the scale in a horizontal configuration were previously-verified by Arends [155]. The calibrations that related to the calculated thrust coefficients in the current experiments were verified here in the horizontal and vertical mounting arrangements

The first set of calibrations was conducted in March 2011 using the scale in the horizontal arrangement. Twenty-four Canadian $1 \$$ coins were used for the calibration and were weighed on a Mettler Toledo AG285 balance with a resolution of $0.1 \mathrm{mg}$. The mass of the coins is shown in Table 3.3 .

The force in the $+F_{z}$ direction was first verified. The calibration procedure began by zeroing the hardware and the software. Coins were then place one at a time at the centre of the balance in position 1 identified above in Figure 3.12. Data was recorded for 10 
Table 3.3: Calibration weights from March 2011 - horizontal orientation.

\begin{tabular}{|c|c|}
\hline Weight Number & Mass [g] \\
\hline 1 & 7.0314 \\
\hline 2 & 6.9677 \\
\hline 3 & 7.0673 \\
\hline 4 & 6.9793 \\
\hline 5 & 6.9227 \\
\hline 6 & 6.9403 \\
\hline 7 & 7.0986 \\
\hline 8 & 6.9943 \\
\hline 9 & 6.9393 \\
\hline 10 & 6.9428 \\
\hline 11 & 6.9999 \\
\hline 12 & 6.9605 \\
\hline 13 & 7.0030 \\
\hline 14 & 6.9980 \\
\hline 15 & 6.9834 \\
\hline 16 & 7.0299 \\
\hline 17 & 7.0599 \\
\hline 18 & 7.0057 \\
\hline 19 & 6.9846 \\
\hline 20 & 6.9718 \\
\hline 21 & 7.0805 \\
\hline 22 & 7.0212 \\
\hline 23 & 6.9974 \\
\hline 24 & 6.9787 \\
\hline
\end{tabular}

$s$ and the average force was calculated each time a new coin was added to the balance. The calibration curve for $F_{z}$ developed using this procedure is shown in Figure 3.14. A linear relationship with a slope of 0.9873 is observed between the actual weight of the coins $\left(F_{z, \text { actual }}\right)$ and the recorded weight output from the balance $\left(F_{z, \text { meas }}\right)$. Additionally. the position sensitivity of the balance was assessed by placing coins 1-5 together at each of the five locations previously-highlighted in Figure 3.12. Forces were recorded for $10 \mathrm{~s}$ at each position and the balance was zeroed in between each test. A bar chart highlighting the insensitivity of $F_{z, \text { meas }}$ to the position of the coins on the platform is shown in Figure 3.15.

The calibration for the moment in the $\mathrm{x}$-direction. $M_{x}$. was verified as $M_{x}$ was used to determine the thrust force in the horizontal configuration. The moments in the positive and negative $M_{x}$ directions were assessed by placing coins at positions 4 and 5 on the balance. respectively. As with the calibration for $F_{z}$. coins were placed one at a time on the balance 


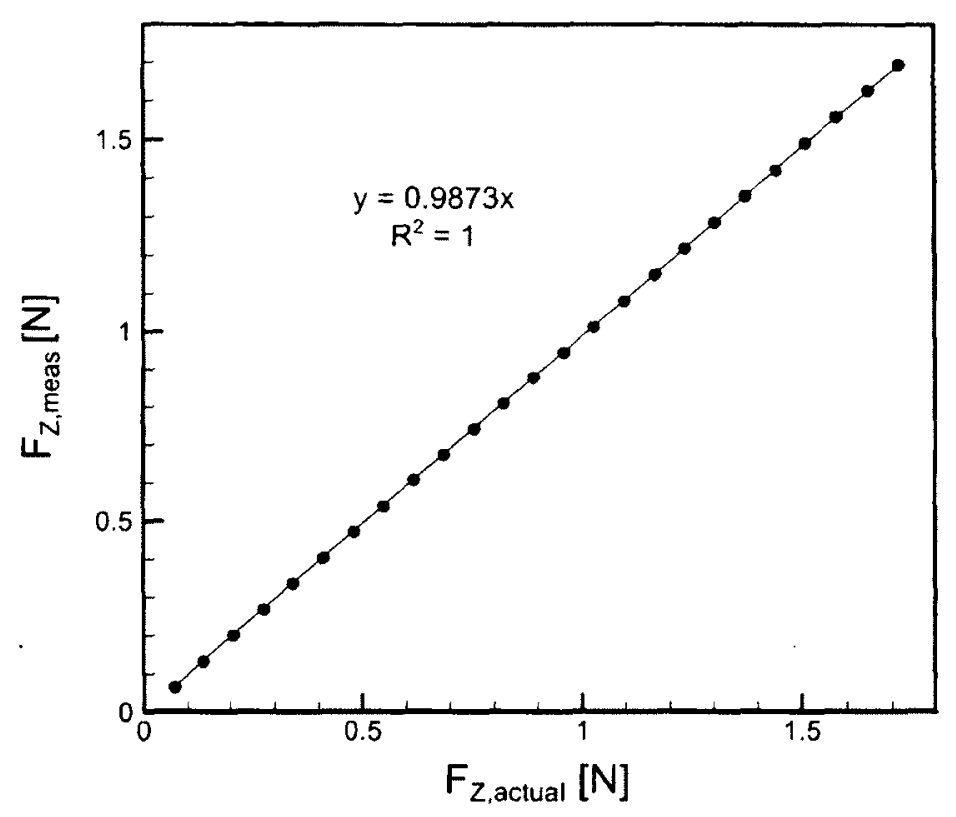

Figure 3.14: Calibration for $F_{z}$ - horizontal configuration.

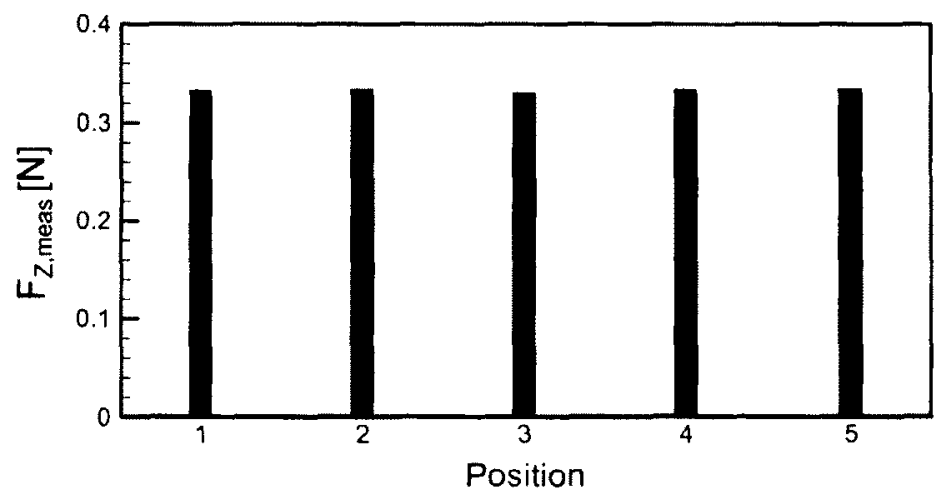

Figure 3.15: Position sensitivity of $F_{z}$ - horizontal configuration.

and averaged moments were calculated based on $10 \mathrm{~s}$ of recorded data. The calibration curve for $M_{x}$ is shown in Figure 3.16 and includes both the positive and negative ranges.

The balance was then calibrated in the vertical orientation using a different set of weights in August 2012. The masses of the weights and the two hangars used to support the weights were obtained using a Mettler Toledo AG285 balance and are shown in Table 3.4. The calibration for $M_{x}$ in the vertical orientation was conducted with a setup that minicked the one used in the water channel. The scale was mounted vertically and a turbine was 


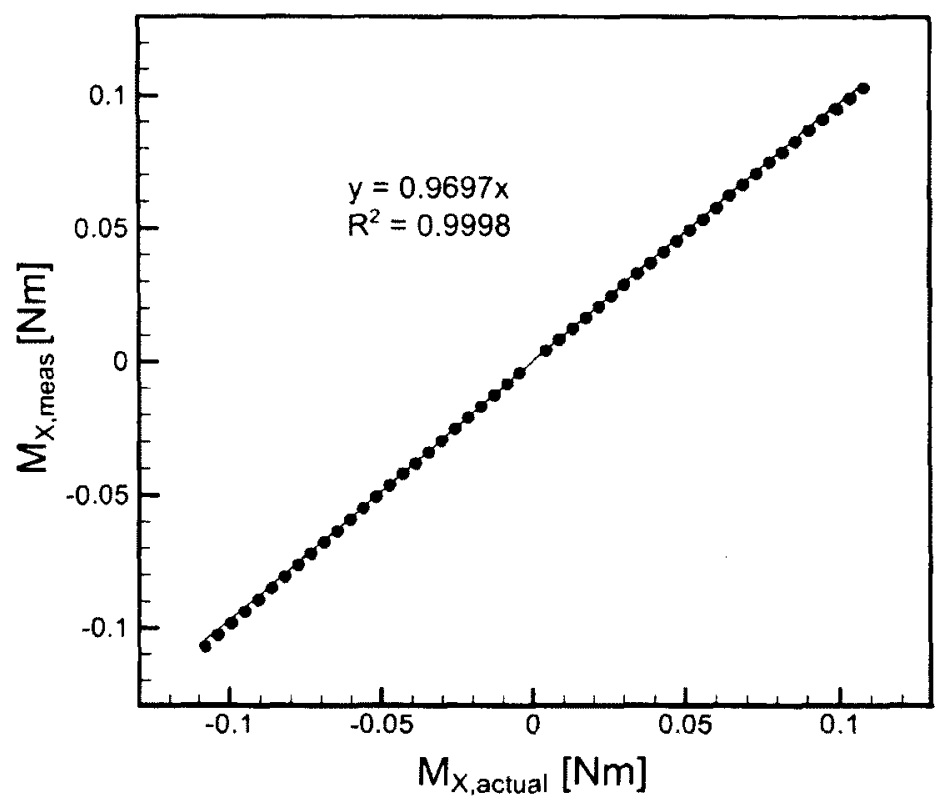

Figure 3.16: Calibration for $M I_{x}$ - horizontal configuration.

attached to the scale using a support strut as indicated in Figure 3.17. The schematic indicates that weights were added to a hangar that was attached to the turbine support strut using a pulley and a piece of string. Weights were added one at a time at the centre of the hangar and the output from the scale was recorded for $10 \mathrm{~s}$ each time a new weight was added. The applied moment was determined based on the force applied to the turbine support strut $\left(F_{z, \text { actual }}\right)$ and the vertical distance, $d$, between the string and the centre of the balance. The applied moment $\left(M_{x, \text { actual }}\right)$ is compared to the moment output by the scale $\left(M_{x, \text { meas }}\right)$ in Figure 3.18 at three different test conditions. The setup was evaluated using a $20 \mathrm{~cm}$ rotor. a $40 \mathrm{~cm}$ rotor, and both the light and heavy weight hangars.

\subsubsection{Blockage corrections and free surface effects}

The presence of an object in a closed test section will create a blockage effect that results in artificial flow acceleration around the object in order to preserve continuity. The definition of blockage used in this thesis is repeated here in Equation (3.7) as the ratio 
Table 3.4: Calibration weights from August 2012 - vertical orientation.

\begin{tabular}{|c|c|}
\hline Weight Number & Mass [g] \\
\hline \hline 1 & 10.2440 \\
\hline 2 & 10.0850 \\
\hline 3 & 10.1540 \\
\hline 4 & 10.1486 \\
\hline 5 & 10.0033 \\
\hline 6 & 10.0015 \\
\hline 11 & 20.0039 \\
\hline 12 & 20.0006 \\
\hline Light hangar & 5.2036 \\
\hline Heavy hangar & 49.9395 \\
\hline
\end{tabular}

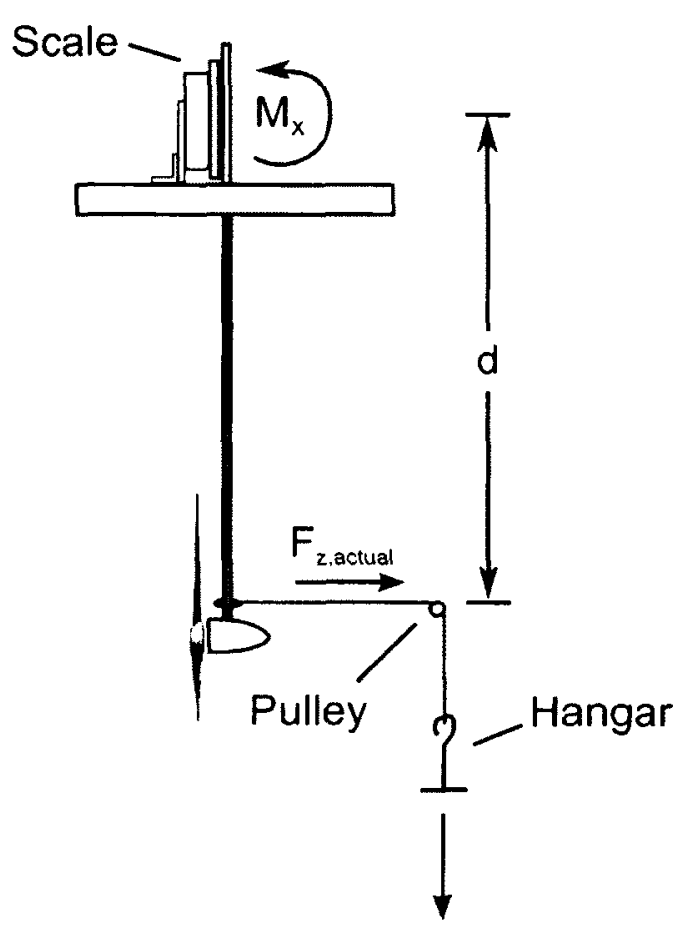

Figure 3.17: Schematic of the vertical calibration setup.

between the swept area of the rotor and the cross-sectional area of the test section. as this is a representative way of characterising blockage caused by dynamic rotor effects and the downstream wake. Blockage will have a tendency to straighten out streamlines around and downstream of an object due to the higher speed. Additionally. blockage will lead to recorded forces and moments which are larger than they would be if the same object was tested in truly freestream conditions due to the local increase in the speed of the flow [156]. 


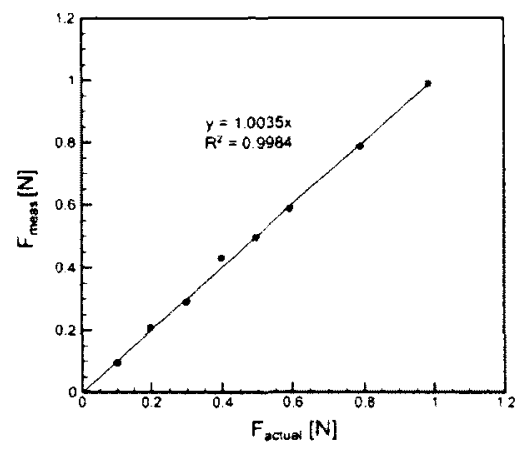

(a) $20 \mathrm{~cm}$ rotor, heavy hangar

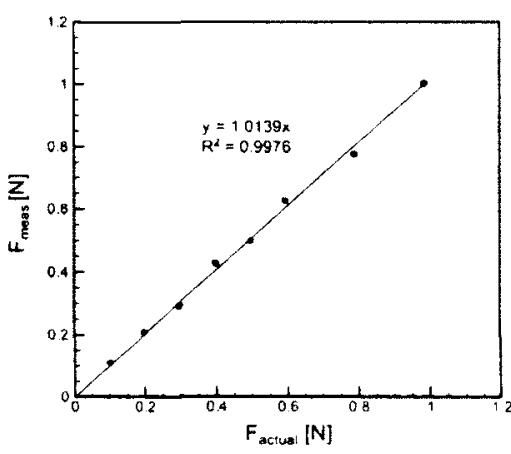

(b) $40 \mathrm{~cm}$ rotor, heavy hangar

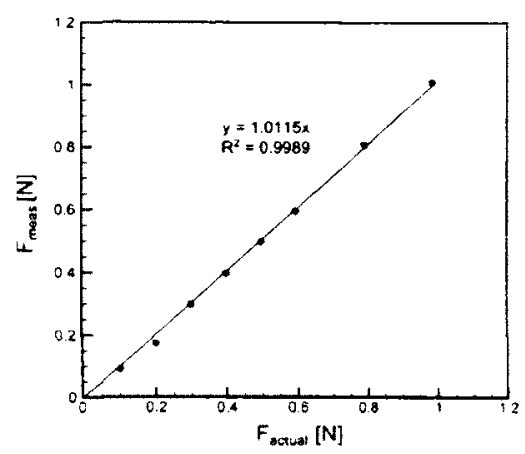

(c) $20 \mathrm{~cm}$ rotor, light hangas

Figure 3.18: Calibration for $M_{x}$ - vertical orientation.

$$
\text { Blockage }=\frac{A_{\text {swept }}}{A_{x}} \times 100
$$

Analytical blockage corrections are typically used to correct thrust coefficients in wind turbine experiments [30.60]. The correction presented by Bahaj et al. in Ref. [30] will be used in the current work to correct thrust and power coefficients for blockage effects. The correction is based on an actuator disc model of a wind turbine in a closed test section and can estimate the equivalent freestream thrust coefficient based on a blockage factor $\left(\epsilon_{B}\right)$.

The symbols and assumptions used in the correction are indicated in the schematic shown in Figure 3.19. The rotor is replaced with an actuator disc, at which the velocity is $U_{1}$ and across which the pressure is discontinuous. Plane $\mathrm{A}$ is located upstream in conditions that are undisturbed by the rotor. where the tunnel speed $\left(U_{T}\right)$ has a uniform profile [30]. Plane B is located downstream where the pressure in and out of the wake is approximately equal. The velocity in the wake of the disc is represented by $U_{2}$ and the downstream velocity outside the wake is $U_{3}$.

By applying continuity and an axial momentum balance. the velocities $U_{1}, U_{2}$, and $U_{T}$ can be related to the crosis-sectional area of the test section $\left(A_{x}\right)$ and $A_{\text {swept }}$ according to 


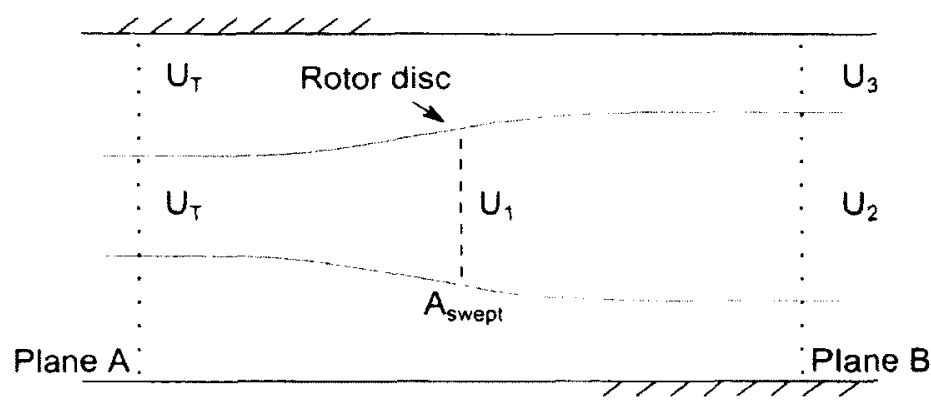

Figure 3.19: Schematic of the actuator disc-based blockage correction.

Adapted from Ref. [30].

Equations (3.8) and (3.9) [30].

$$
\begin{gathered}
\frac{U_{1}}{U_{2}}=\frac{-1+\sqrt{1+\frac{A_{s w e p t}}{A_{x}}\left[\left(\frac{U_{3}}{U_{2}}\right)^{2}-1\right]}}{\frac{A_{\text {swept }}}{A_{x}}\left[\left(\frac{U_{3}}{U_{2}}\right)-1\right]} \\
\frac{U_{T}}{U_{2}}=\frac{U_{3}}{U_{2}}-\frac{A_{\text {swept }}}{A_{x}} \frac{U_{1}}{U_{2}}\left[\frac{U_{3}}{U_{2}}-1\right]
\end{gathered}
$$

The rotor's uncorrected thrust coefficient can also be rewritten as

$$
C_{T, U}=\frac{F_{\text {Thrust }}}{0.5 \rho A_{\text {swept }} U_{T}^{2}}=\left(\frac{U_{2}}{U_{T}}\right)^{2}\left[\left(\frac{U_{3}}{U_{2}}\right)^{2}-1\right]
$$

Equation (3.10) can be rearranged to produce Equation (3.11).

$$
\frac{U_{T}}{U_{2}}=\frac{1}{\sqrt{\frac{C_{T . U}}{\left(\frac{u_{3}}{U_{2}}\right)^{2}-1}}}
$$

The benefit of using this formulation is that the velocities in the test section do not need to be known. as only the velocity ratios are required. In order to solve the equations. a range of values are selected for $U_{3} / U_{2}$ and Equations (3.9) and (3.11) are solved iteratively 
until convergence is achieved. Once the two equations have converged to a common value for $U_{T} / U_{2}$, the velocity ratio $U_{1} / U_{T}$ can be determined by first calculating $U_{1} / L_{2}$ with Equation (3.8) and then multiplying the result with the inverse of $U_{T} / U_{2}$. The ratio $l_{1} / C_{T}$ can then be used to determine the relationship between the flow speed in the tunnel and the equivalent freestream conditions using Equation (3.12).

$$
\frac{U_{T}}{U_{F}}=\frac{\frac{U_{2}}{U_{T}}}{\left(\frac{U_{1}}{U_{T}}\right)^{2}+\frac{C_{T . U}}{4}}
$$

The velocity $U_{T} / U_{F}$ represents the blockage factor $\left(\epsilon_{B}\right)$ and can be expressed more conveniently as

$$
\epsilon_{B}=\frac{U_{1}}{U_{T}}+\frac{U_{T}}{U_{1}}\left(\frac{C_{T, U}}{4}-1\right)
$$

The blockage factor can then be used to correct the uncorrected thrust coefficients recorded in the closed test section $\left(C_{T, U}\right)$ to their equivalent freestream conditions in the absence of blockage using Equation (3.14) [30].

$$
C_{T, C}=C_{T, U} \epsilon_{B}^{2}
$$

Water channel experiments differ from wind tunnel experiments in that there are three solid walls and one free surface. The free surface facilitates access to a model in a water channel, but it may affect the development of the flow around an object.

A similarity parameter that should be considered when matching a water channel experiment to a channel flow or surface wave problem is the Froude number $(F r)$. The Froude number is the relationship between the freestream speed and the surface wave propagation speed in channel flows and is defined by Equation (3.15). where $U_{x}$ is the freestream speed. $g$ is gravity. and $z$ is the height of the free surface in the channel. 


$$
F r=\frac{U_{\mathrm{x}}}{\sqrt{g z}}
$$

The Froude number is analogous to the Mach number in air as it relates to the direction of propagation of flow information. The non-dimensional number should be maintained in the subcritical flow regime in channel flow $(<1)$ if an analogy is to be maintained with subsonic flow in air. Given that the water channel was not being used to match a particular surface wave condition, it was desired to maintain a low Froude number such that the free surface of the water was not disturbed during the experiments. The Froude number in the current experiments will be discussed briefly in Chapter 4, but ranged from 0.036 to 0.082 and was in the subcritical flow regime.

In tidal turbine experiments. the Froude number can typically reach $0.2-0.3$ and the free surface of the water is affected by the presence of the rotor. A two-dimensional model to combine blockage and surface effects was proposed by Whelan et al. in 2009 [157]. A schematic of the model has been adapted from Ref. [157] in Figure 3.20. The model assumes that the free surface of the water will drop downstream of the rotor. This was caused by the presence and energy extracting nature of the rotor. As a result of the drop in height of the free surface, the speed of the bypass flow $\left(U_{b}\right)$ around the rotor was assumed to increase further and it was proposed in Ref. [157] that forces and moments may experience an increase greater than what would be observed with traditional blockage effects.

The model of Whelan was evaluated in a separate work by Shives and was found to overestimate the drop in the free surface [73]. Shives developed an alternate model based on CFD simulations of a tidal turbine represented with an actuator disk. The resulting model is highlighted in Equations (3.16). (3.17), and (3.18). The inputs to the model are the thrust coefficient $\left(C_{T}\right)$. the blockage ratio $\left(B_{r}\right)$ and the Froude number of the flow: 


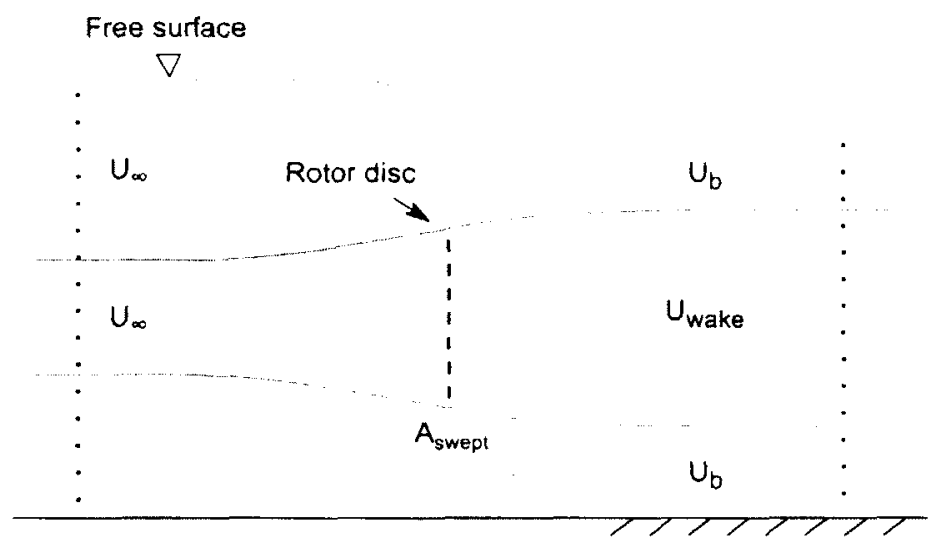

Figure 3.20: Schematic of the free surface variation due to blockage.

Adapted from Ref. [157].

$$
\begin{gathered}
\frac{h_{6}}{h_{0}}=2 \sqrt{\frac{-p}{3}} \cos \left\{\frac{1}{3} \arccos \left[\frac{3 q}{2 p} \sqrt{\frac{-3}{p}}\right]\right\} \\
p=\left[\left(C_{T} B_{r}-2\right) F r^{2}-1\right] \\
q=2 F_{r}^{2}
\end{gathered}
$$

\subsection{Wind tunnel experiments}

The wind farm layout experiments were conducted in the atmospheric boundary layer (ABL) wind tunnel at Carleton University. The wind tunnel geometry. the design of the scaled wind turbines. and the wind turbine layouts that were considered will be described. Additionally, the flow conditioning elements used to generate a scaled ABL will be presented. The instrumentation and setup of the three-axis traverse will then be highlighted. Lastly. the calibration procedures and data acquisition parameters used in conjunction with the hot-wire probe measurements will be described.

The ABL tunnel at Carleton is an open-circuit wind tunnel designed for experiments 
occurring in scaled boundary layer flows and can be seen in Figure 3.21. The wind tunnel has an upstream contraction ratio of $7.1: 1$ followed by a fetch distance of $9.76 \mathrm{~m}$. The turbine is powered by a $30 \mathrm{~kW}$ motor coupled to a $1.67 \mathrm{~m}$ diameter fan and this allows the tunnel to reach a maximum flow speed of $17 \mathrm{~m} / \mathrm{s}$.

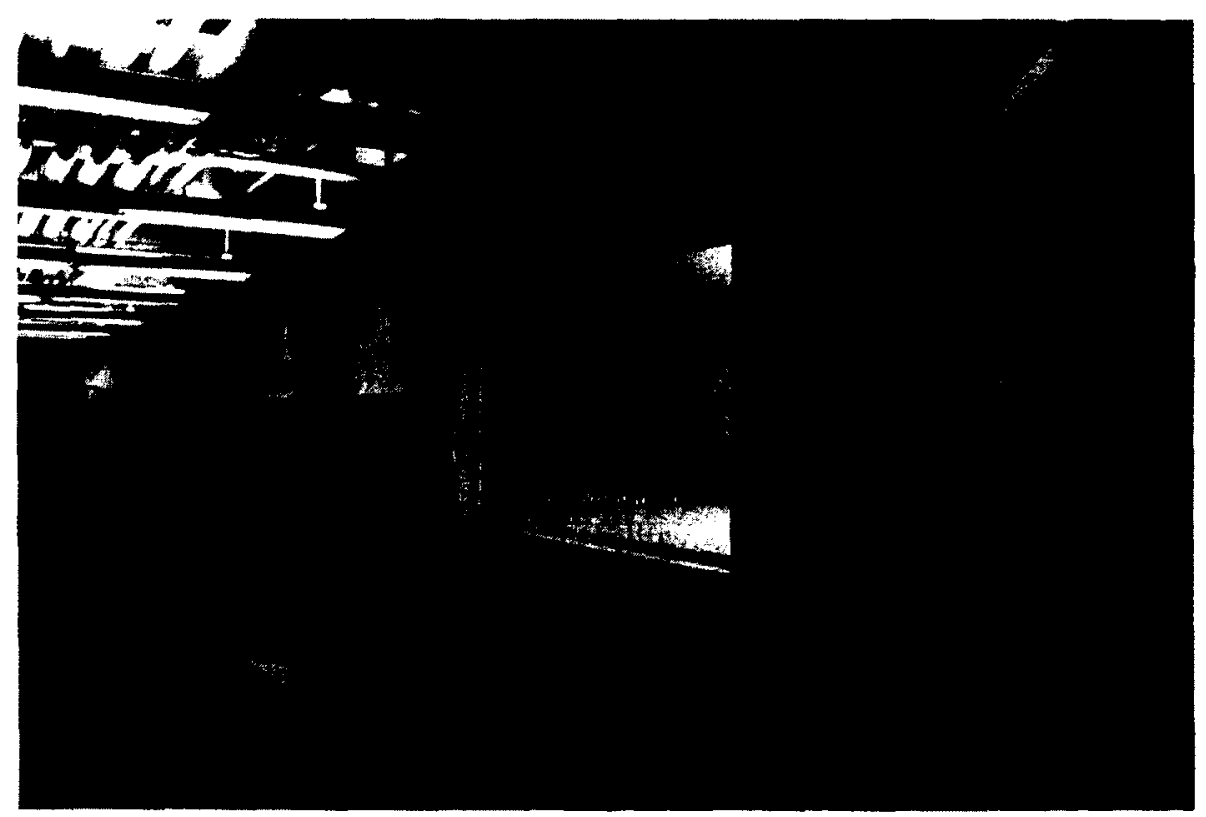

Figure 3.21: The open-circuit ABL tunnel at Carleton University.

The wind tunnel was commissioned in 1979 and has been used throughout the past 30 years for a wide range of experiments, including snow drift interaction with the roof of Montreal's Olympic Stadium and vortex interactions with building-mounted solar panels $[155,158]$. Images of the Olympic Stadium in the ABL tunnel are shown in Figure 3.22.

A drawing of the boundary layer wind tunnel in $2140 \mathrm{ME}$ at Carleton University has been reproduced from Professor Kind's research notes and has been adapted in Figure 3.23 to reflect the current state of the wind tunnel room [158]. The dimensions in Figure 3.23 are in imperial units for consistency with Kind 's research notes and to reflect the system of units used when the tunnel was built. Metric equivalents were not provided in the drawing for clarity. A closed-return, two-dimensional wind tunnel with an offset return 

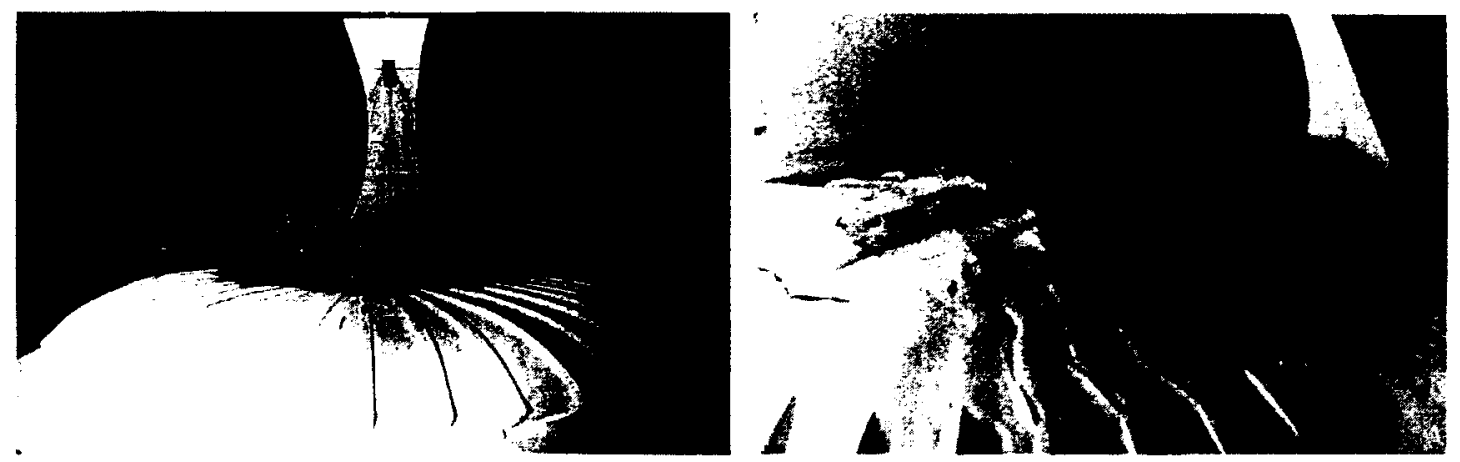

Figure 3.22: Snow drifting studies conducted with a scaled model of Montreal's Olympic Stadium [158].

leg was commissioned in the same room in 2005 in order to study rotorcraft blade-vortexinteractions (BVI). The BVI tunnel was built on the exterior wall side of $2140 \mathrm{ME}$ and blocks a significant portion of the return for the open-circuit ABL tunnel. Additionally, an open passage was been created between the wind tunnel room and the motor control room in 2010 that modified the shape of the room. 

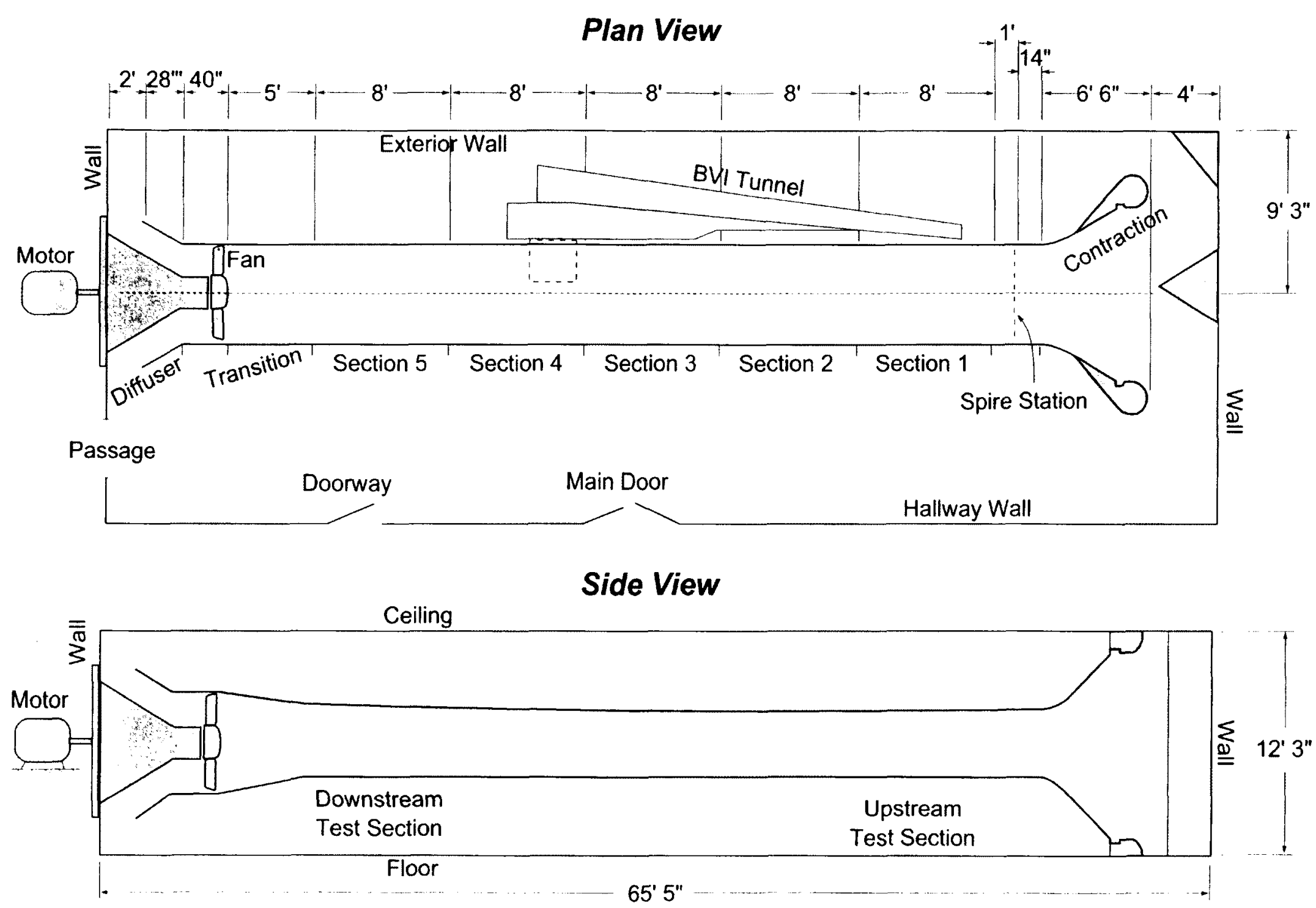

Figure 3.23: Drawing of the ABL wind tunnel room. Adapted from Kind [158]. 
The ABL tunnel was designed with upstream and downstream test sections. which both have a height of $1.12 \mathrm{~m}$. a width of $1.68 \mathrm{~m}$. and a length of $2.44 \mathrm{~m}$ (44 in $\times 66$ in $\times$ $8 \mathrm{ft}$ ). Access is provided to each test section by a horizontally-hinged plexiglass door. The upstream test section is used for experiments in clean uniform flow conditions. An experiment was conducted in the upstream section with a single rotor and the test section was configured with a smooth floor and without boundary layer spires.

The wind farm experiments were conducted in the downstream test section. In this configuration, boundary layer spires were installed at the upstream spire station indicated in Figure 3.23 and roughness panels were installed along the full fetch distance of the tunnel, corresponding to Sections 1-4 in Figure 3.23. The test section floor of the wind tunnel is typically configured with a turntable. but the floor was replaced for the current experiments. A matrix of holes were drilled in the tunnel floor that corresponded to the prescribed positions for the turbines used in the various lateral and longitudinal wind farm configuration experiments. Figure 3.24 identifies the potential turbine locations. The dimensions are normalised with respect to the $20 \mathrm{~cm}$ diameter wind turbine blades and a downstream distance of 0 corresponds to the upstream measurement plane used in the experiment. The nomenclature for the points in Figure 3.24 was used in file naming conventions where $P$ refers to the Port side of the tunnel, $S$ refers to the Starboard side of the tunnel, $U$ was an Upstream position. and $C$ refers to positions along the Centreline.

\subsubsection{Atmospheric boundary layer development}

Wind turbines operate in the atmospheric boundary layer. which causes fluctuating turbine loading. The full-scale $A B L$ can have a height that ranges from $200 \mathrm{~m}$ to over 1 $\mathrm{km}$ and has a profile that depends on atmospheric stability and the roughness of the local topography [159]. The open circuit ABL wind tunnel at Carleton University was used in the 


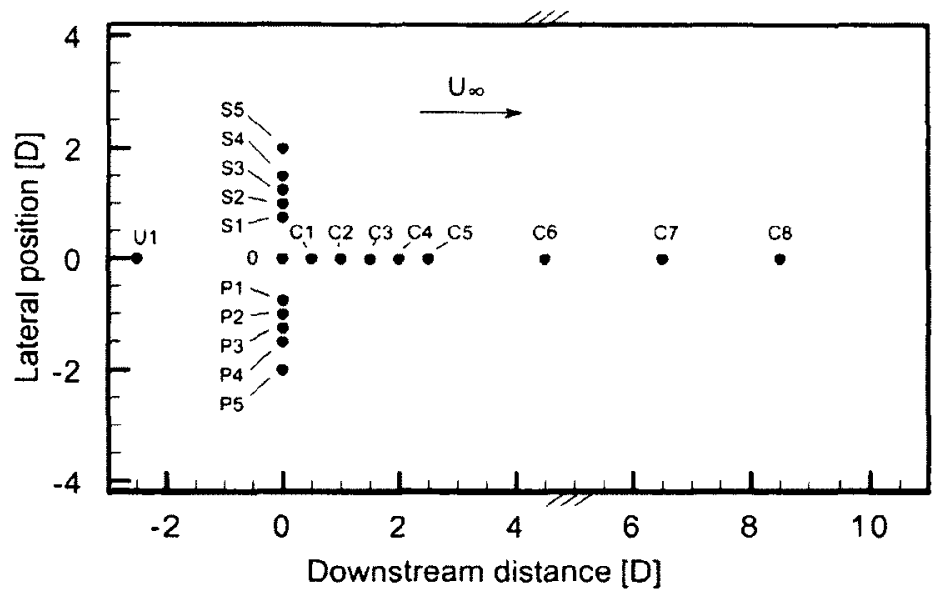

Figure 3.24: Layout of the wind turbine positions.

current study in order to simulate the effects of a neutral atmospheric boundary layer. A scaled boundary layer was created in the wind tunnel using triangular spires and distributed roughness elements along the floor of the wind tunnel, as seen in Figure 3.25(a).

The boundary layer spires and the roughness elements were designed using the empirical sizing methodology described by Irwin [160]. This approach assumes that a uniform velocity profile is found upstream of the spires and that the wind tunnel has a rectangular crosssection with a height $H[160]$. A boundary layer with a height $\delta$ will develop downstream of the spires with the power law profile recalled here in Equation (3.19), where $U$ is the speed at a particular height $z . U_{\delta}$ is the speed at a reference height $\delta$. and $\alpha$ is the desired power law exponent. The triangular spires installed at the test section inlet are equally-spaced in the lateral direction and their base $b$ and height $h$ dimensions are sized according to the boundary layer height and profile desired for a particular application.

$$
\frac{U}{U_{\delta}}=\left(\frac{z}{\delta}\right)^{a}
$$

Once the desired $\delta$ and $\alpha$ have been selected. the height of the spires can be calculated 


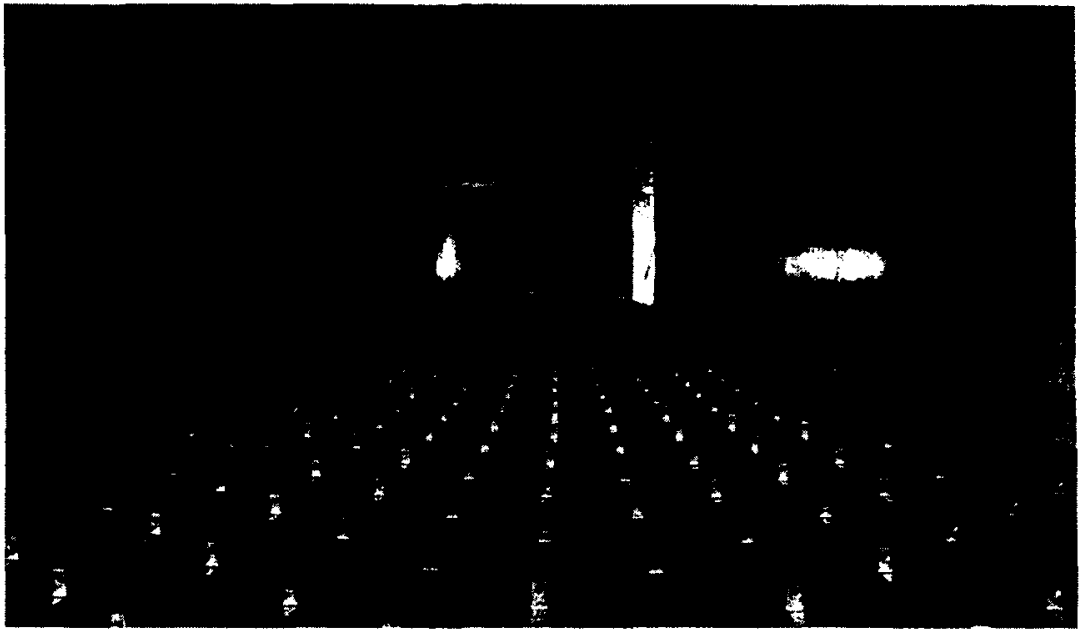

(a) Upstream view of the flow conditioning elements.

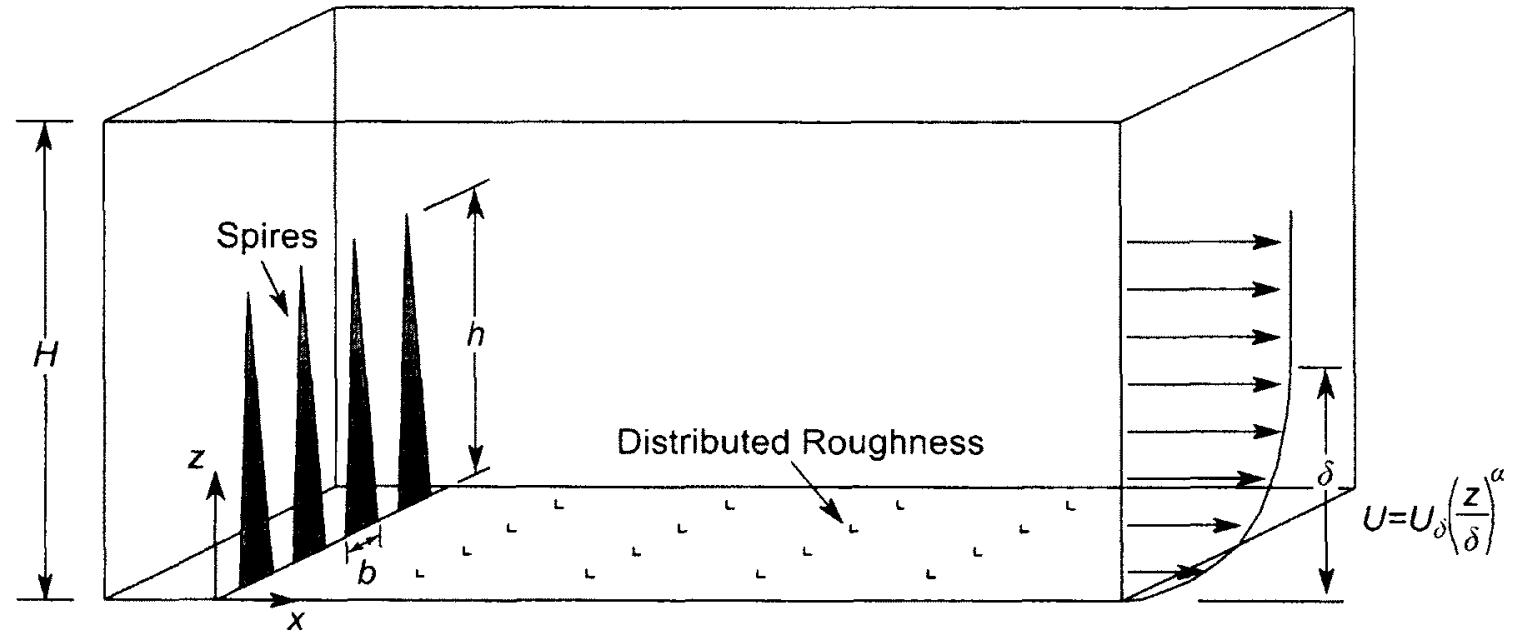

(b) Schematic of the spires and boundary layer. Adapted from Irwin [160].

Figure 3.25: Flow conditioning elements in the wind tunnel.

using Equation (3.20)

$$
h=\frac{1.39 \delta}{1+\alpha / 2}
$$

The base-to-height ratio of the spires can subsequently be determined as

$$
b / h=0.5\left[\frac{c(H / \delta)}{1+c}\right](1+\alpha / 2)
$$


where two additional coefficients $c$ and $d$ can be determined with Equations (3.22) and (3.23).

$$
\begin{gathered}
c=\frac{d\left\{\left[\frac{2}{1+2 \alpha}\right]+d-\left[\frac{1.13 \alpha}{1+\alpha}(1+\alpha / 2)\right]\right\}}{(1-d)^{2}} \\
d=\frac{\alpha(\delta / H)}{1+\alpha}
\end{gathered}
$$

Equation (3.21) includes the contribution of the drag from a roughened floor downstream of the spires necessary to produce a boundary layer with the desired $\delta$ and $\alpha$. The friction coefficient $\left(C_{f}\right)$ that would produce these boundary layer characteristics can be approximated using Equation (3.24).

$$
C_{f}=1.36\left[\frac{\alpha}{1+\alpha}\right]^{2}
$$

The roughness element size that is required to produce the necessary floor skin friction coefficient can be estimated using the empirical relationship described in Equation (3.25). The spacing of the roughness elements $(D)$ must be selected in order to determine the roughness element height $(k)$. The overall shape of the roughness elements is not prescribed in Ref. [160], and various authors have used wooden cubes. spanwise chains, or metal Lbrackets $[16.24,158]$.

$$
k / \delta=\exp \left\{(2 / 3) \ln (D / \delta)-0.1161\left[\left(2 / C_{f}\right)+2.05\right]^{1 / 2}\right\}
$$

The empirical formulation described by Irwin assumes that the desired boundary layer height and profile will be formed at a distance $6 h$ downstream of the spires. If the test section is longer than this, then Equation (3.26) can be used to determine the additional boundary layer thickness that would be achieved by an additional $\Delta x$ downstream fetch 
distance.

$$
\Delta \delta=0.068 \alpha\left[\frac{1+2 a}{1+a}\right] \Delta x F
$$

The function $F$ in Equation (3.26) can be estimated using Equation (3.27). $F$ is a correction factor that accounts for the pressure drop in the test section caused by the boundary layer growth [160].

$$
F \simeq\left\{1+(\delta / H)\left[\frac{\alpha(3+2 \alpha)}{1+\alpha(1-\delta / H)}\right]\right\}^{-1}
$$

Based on the sizing described by Irwin. two sets of spires were selected for the wind tunnel experiments [160]. It was desired to produce scaled boundary layers that were representative of rough, forested terrain and of smooth. offshore conditions. A rough boundary layer corresponding to a power law profile with $\alpha=0.25$ could be represented well with existing spires that had a height of $83.8 \mathrm{~cm}$ and a width of $12.7 \mathrm{~cm}(33 \mathrm{in} \times 5 \mathrm{in})$. The original roughness panels designed by Kind were broken and unusable and therefore new panels of roughness elements were designed based on the methodology of Irwin [160]. Sizing indicated that the roughness elements should have a height of $3 \mathrm{~cm}(1.2 \mathrm{in})$. but this size would have necessitated manufacturing custom elements. As an alternative. $2.54 \mathrm{~cm}$ (1 in) angle brackets were purchased off-the-shelf and were glued to $1 / 8$ in thick hardboard panels. Sizing for a smooth boundary layer profile with $\alpha=0.1$ indicated that spires with a height of $80.0 \mathrm{~cm}$ and a width of $5.1 \mathrm{~cm}$ could produce the desired boundary layer profile.

\subsubsection{Wind turbines}

The wind tunnel experiments were all conducted using $20 \mathrm{~cm}$ diameter MAAE rotor blades. The size of the blades was based on the size of the wind tunnel and the height of the boundary layer that could be generated within the tunnel. The towers for the wind 
turbines were printed at Carleton University using the Dimension BST three-dimensional printer. A DC generator was friction fit into the hub of each tower and the centre of rotation was $20 \mathrm{~cm}$ from the wind tunnel floor. This provided a blade clearance distance of $0.5 \mathrm{D}$ between the lowest blade tip position and the floor of the wind tunnel, consistent with the ratio observed on a number of commercial onshore wind turbine designs.

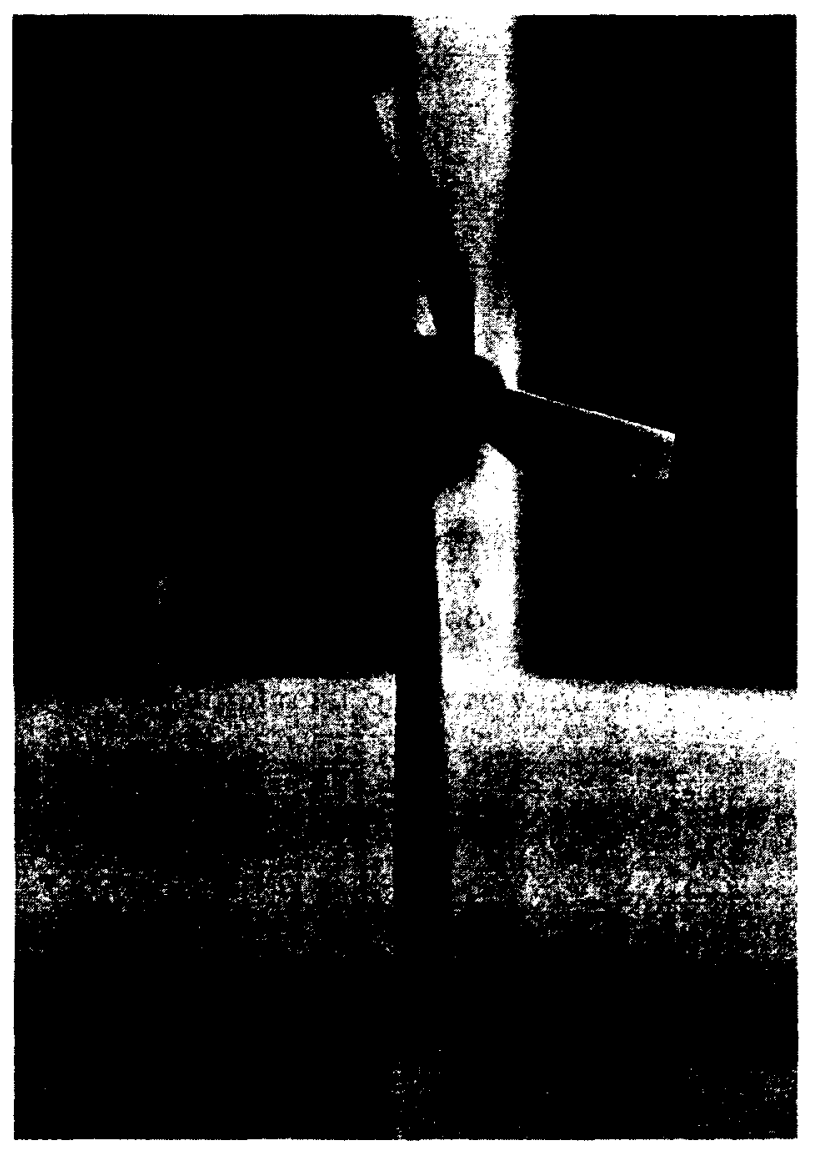

Figure 3.26: Wind turbine model in the wind tunnel.

The low-torque DC generators were obtained from the company KidWind and were each wired to a 10 -turn. $5 \mathrm{k} \Omega$ potentiometer in parallel with a $4.7 \mu \mathrm{F}$ capacitor. The load provided by the potentiometers allowed the rpm of each turbine to be controlled independently. The voltage drop recorded by the data acquisition system $(V)$ and the resistance provided by the potentiometer $(\Omega)$ were used to calculate the electrical power $\left(P_{\text {elec }}\right)$ generated by the turbine using Equation (3.28). 


$$
P_{\text {elec }}=\frac{V^{2}}{\Omega}
$$

A similar method of controlling the power output and rotational speed of small wind turbine models has been used by Chamorro et al. by Corten et al., and by Ozbay [16. $64,77,79\}$. The baseline rotor operating conditions had a $\lambda \approx 6$ and $R e_{\text {tip }} \approx 38.000$ and this corresponds to a rotational speed of $3300 \mathrm{rpm}$. The rotational speed of the turbine could not be precisely maintained (as would be the case in a fixed-speed commercial wind turbine). and so the actual rotational speed of the rotor will fluctuate with turbulence in the boundary layer and changes in the mean freestream speed. This also means that turbines operating in the downstream wake of an upstream turbine will experience a slower inflow speed and will rotate with a slower rotational speed if the load provided by the resistor was maintained. A lower rotational speed and an associated lower voltage output would be divided by the same $\Omega$, leading to a decrease in power. An alternative that was evaluated would involve adjusting the load on the generator for downstream turbines such that the target rotational speed could be maintained. In this case, the target rotational speed and voltage would be divided by a larger value of $\Omega$, leading to approximately the same power output as in the preceding case. The logistical challenges associated with this approach were cumbersome, and the goal of the experiment was not to match a particular rotational speed. Additionally. the overall outcome regarding the power output of the rotor was similar in both cases (allow rpm to vary for a fixed load. or attempt to match rpm by changing the load).

Therefore. the approach that was selected involved setting $\Omega$ for each turbine such that the individual. isolated turbines operated with $\lambda \approx 6$ and $R e_{\text {tip }} \approx 38.000$. The rpm was then allowed to fluctuate at a constant load regardless of the wind farm configuration being tested. consistent with the approach selected in Refs. [16.64,77.79]. 


\subsubsection{Instrumentation and data acquisition system}

The primary instrumentation and equipment used in the wind farm experiments can be seen in Figure 3.27. A computer with a $3 \mathrm{GHz}$ Pentium 4 processor and $512 \mathrm{MB}$ RAM used Labview 2010 version 10.0 to interface with the data acquisition system (DAQ) and the motor controller. Data were acquired using a National Instruments (NI) CompayDAQ system with a NI-9205 analog input module. The DAQ can support 32 single-ended channels, 16 differential channels, or a combination thereof and each channel had a range of $\pm 10 \mathrm{~V}$. The module has 16 -bit resolution and a maximum sampling rate of 250,000 samples per second.
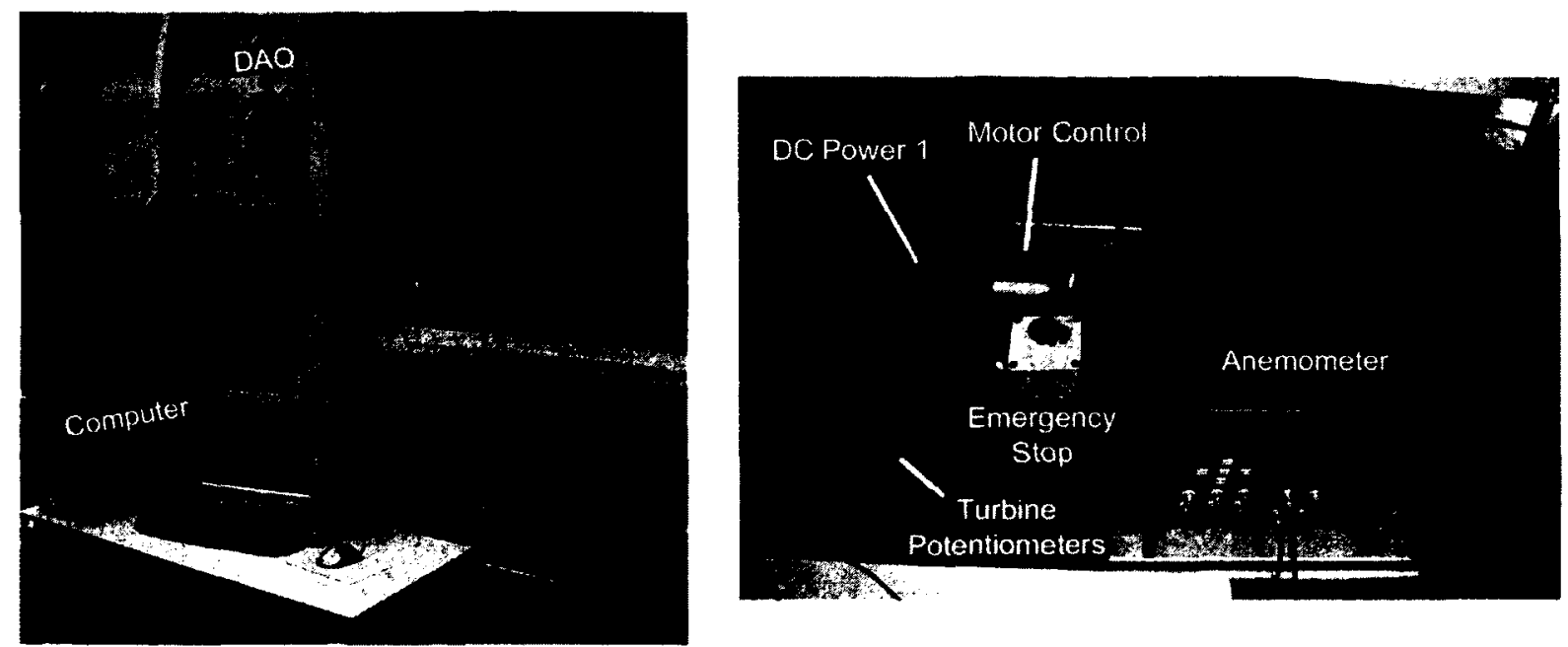

Figure 3.27: Photograph of the primary instrumentation and equipment.

The NI-9205 data acquisition module is shown in Figure 3.28 along with the wiring setup used in the current experiment. The DAQ is also used to acquire input signals from the Department's scaled wind farms and therefore 16 single-ended channels were configured for turbine voltages. All of the analog inputs for the current experiment (pressure transducer. string potentiometer, three turbines, and the anemometer) were wired as differential inputs. While the DAQ has analog inputs for either 32 single-ended channels or 16 differential channels, row 17 includes a common ground for single-ended reference measurements and 
row 18 includes a single digital output channel.
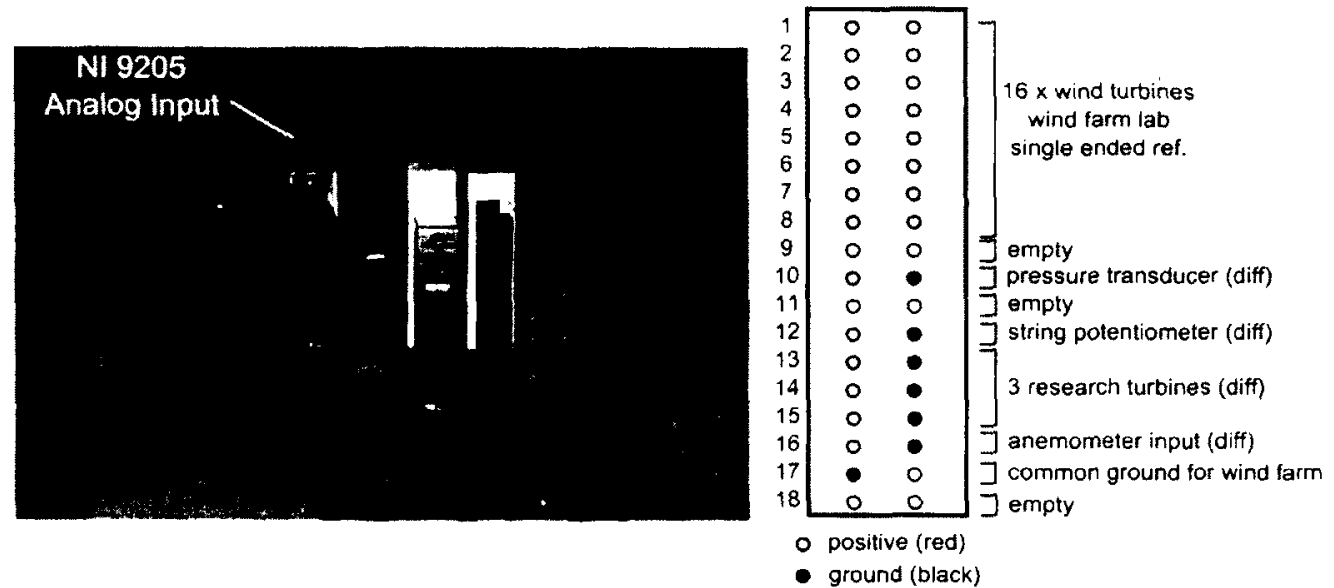

Figure 3.28: The NI Compaq-DAQ system and wiring diagram.

The ambient temperature and humidity in the wind tunnel room were monitored using a thermocouple module and a humidity module that are input to the hot-wire anemometer. The J-type thermocouple was mounted two metres away from the anemometer in a quiescent environment and the sensed temperature was output on the digital display of the anemometer. The temperature was recorded during each experiment with the hot-wire probe with a precision of $0.1^{\circ} \mathrm{C}$.

Two power supplies were used during the experiment. The first power supply was a RS75-24 24 VDC switching power supply from Mean Well that was used to power the traverse motor, the pressure transducer, and the motor controller. The second power supply was a steady DC power supply from BK Precision. Model 1610. (S/N 145-04858) and was used to power the string potentiometer as described below.

The Pitot-static tube used to determine the reference speed used to set the tunnel operating conditions was a 160-36 Pitot-static tube from Furneco (Dwyer Canada). A PX653 pressure transducer from Omega (Serial X11080025) was used to convert the pressure sensed by the Pitot-static tube into a voltage. The transducer has a measurement range from 0 to 0.25 inches of water $(62 \mathrm{~Pa})$ and has an output voltage between 1 and $5 \mathrm{~V}$. The 
available range of the transducer corresponds to a maximum flow speed of $10 \mathrm{~m} / \mathrm{s}$. The transducer was pre-calibrated by the manufacturer and the calibration curve is shown in Figure 3.29 .

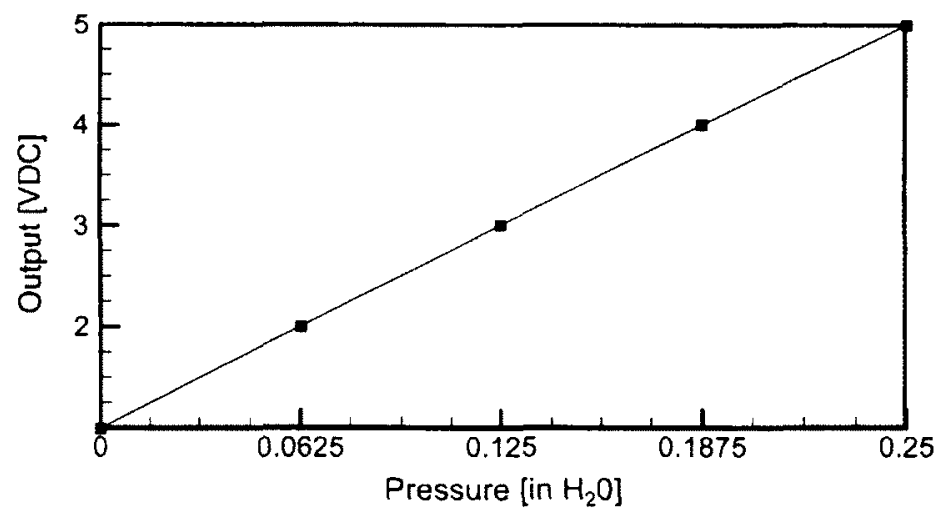

Figure 3.29: Calibration curve for the PX653 pressure transducer.

The lateral position of the hot-wire probe in the test section was determined using a string potentiometer that was attached to the actuator cart of the traverse system. A string potentiometer consists of a thin metal cable integrated into a potentiometer and outputs a voltage that is proportional to the extension of the cable. The LX-PA-50 series string potentiometer from UniMeasure (Serial number 42050470) used in the experiment had a maximum range of $1270 \mathrm{~mm}$. The output voltage of the string potentiometer $\left(V_{\text {out }, s p}\right)$ is ratiometric and will depend on the excitation voltage $\left(V_{\ell}\right)$ applied to the sensor. The sensor has a maximum excitation voltage of $25 \mathrm{~V}$, but the excitation voltage was maintained at $10.00 \mathrm{~V}$ throughout the experiments so as not to exceed the range of the DAQ. The string potentiometer had initially been powered by a $10 \mathrm{~V}$ switching-type DC power supply. but this produced an output voltage that switched between $V_{\text {out,sp }}$ and 0 at the frequency of the power supply. As this was not satisfactory, the sensor had to be powered by a separate, steady DC power supply, referred to as DC Power 2 in the following subsection. The manufacturer-provided sensor sensitivity was $0.77194 \mathrm{mV} / \mathrm{mm} / V_{e}$ which produced a calibration curve for the string potentiometer distance $\left(d_{s p}\right)$ according to Equation (3.29) when $V_{e}=10.00 \mathrm{~V}$. 


$$
d_{s p}[\mathrm{~mm}]=128.67 V_{\text {out }, s p}
$$

This calibration was verified in situ on the traverse setup by conducting three separate calibrations. The traverse was moved toward the starboard side of the test section in intervals of approximately $\Delta x \approx 10 \mathrm{~mm}$ and then was stepped back towards the port side of the tunnel in approximately $20 \mathrm{~mm}$ intervals. The distance moved by the string potentiometer was measured at each interval and is compared to the $V_{\text {out,sp }}$ in Figure 3.30. The slope of the calibration equation resulting from this procedure varied from the manufacturer-provided slope by approximately $1 \%$. The calibration procedure was repeated 2 additional times, and the new calibration equation resulting from the three combined data sets is indicated in Equation (3.30).

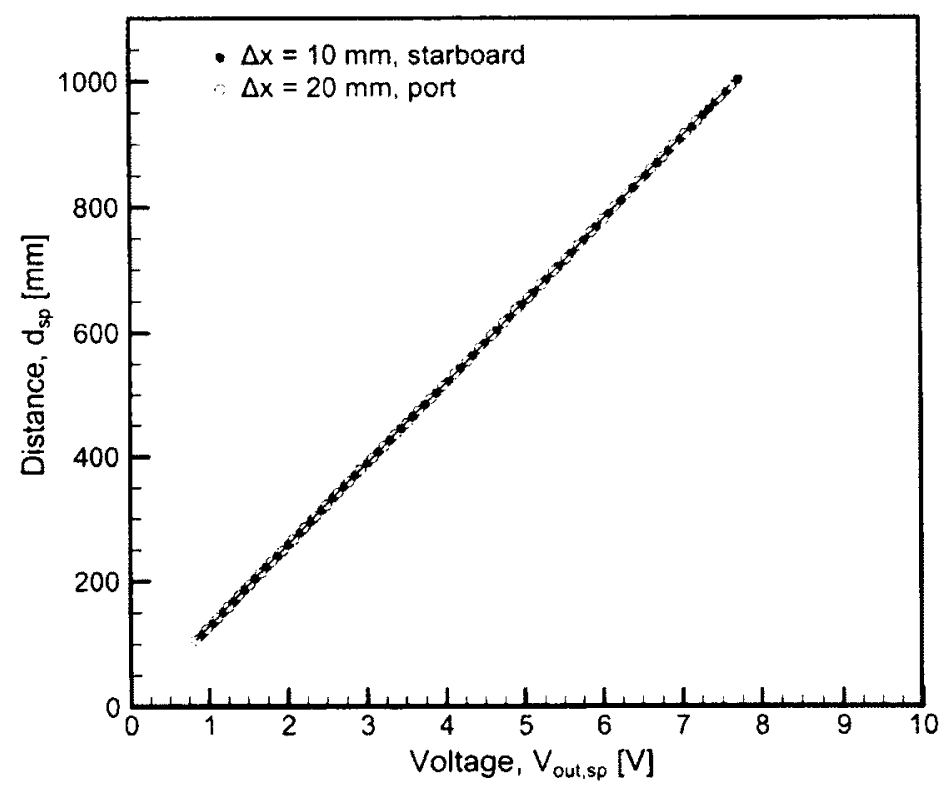

Figure 3.30: Manual calibration curve for the string potentiometer.

$$
d_{s p}[\mathrm{~mm}]=130.5 V_{\text {out }, s p}-3.29
$$




\subsubsection{Traverse design}

One of the goals of the planned experiment was to determine the flow speed downstream of a variety of wind farm configurations. A three-axis traverse system was therefore designed in order to map the flow in the region of interest. The majority of the traverses that were planned were lateral traverses at the turbine hub height, and so the lateral (spanwise) axis was the only automated direction of travel in order to keep the design straightforward and inexpensive. The probe height could be adjusted manually in the vertical and streamwise directions in order to infer the flow speed at various planes. An image of the traverse system that was manufactured can be seen in Figure 3.31. The traverse system was mounted on streamwise-aligned rails and can be moved upstream or downstream. The traverse actuator is mounted on a plate which is attached to bearing blocks so that the entire assembly can be easily moved along the rails. A latch-style brake was installed alongside the bearing blocks on both rails to lock the assembly in place during a particular test. The vertical axis is controlled manually by adjusting a guide on a Vernier scale. The Vernier scale had a resolution of $0.001 \mathrm{ft}(0.3 \mathrm{~mm})$ and a set screw was used to fix the vertical axis at a particular height above the test section floor.

The lateral motion of the traverse system was automated using a FA-200-TR-24-50 track actuator from Firgelli Automations. The screw-driven actuator had a range of motion of $1270 \mathrm{~mm}$ (50 in). The actuator's motor was controlled using a model 1065, $5 \mathrm{amp} .9-28 \mathrm{~V}$ brushed DC motor controller from Phidgets. The actuator comes with a pre-installed cart that was used to fasten the vertical axis to the actuator. The string potentiometer was fastened on the port side of the traverse system and was installed in such a way that the aluminum extrusion of the track actuator shielded the potentiometer's cable from the flow. The end of the cable was fastened to the actuator cart so that motion of the cart would modify the output of the potentiometer. 


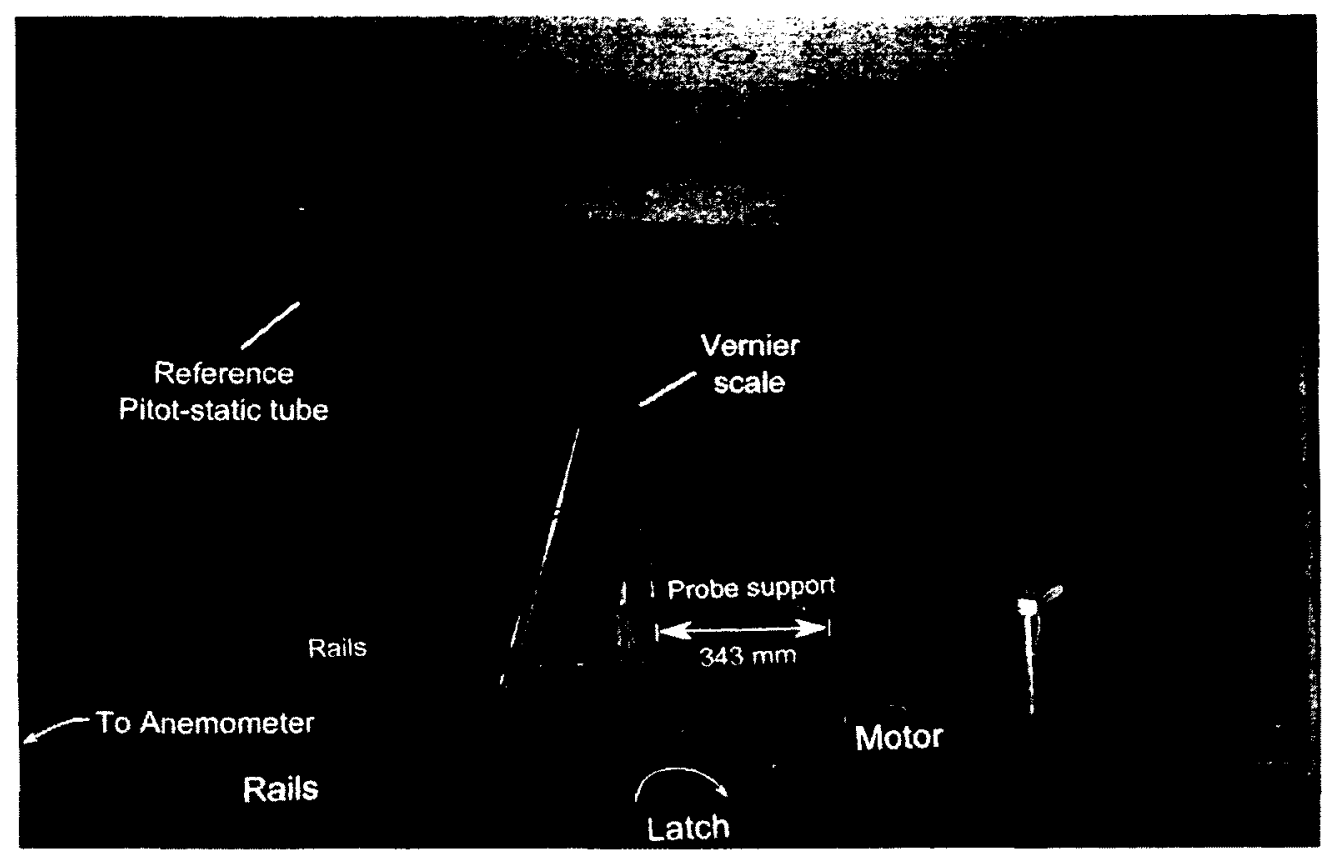

Figure 3.31: Photograph of the traverse equipment.

The actuator had pre-installed limit switches. but their locations were not compatible with the location of the string potentiometer. Two additional contact switches were therefore installed on the frame of the actuator to prevent damage to the actuator. the motor, and the string potentiometer. The contact switches would cut power to the motor when activated and these were wired to an additional emergency stop button located beside the data acquisition computer. A schematic of the wiring setup for the traverse system is shown in Figure 3.32. Connectors were used where possible so that the traverse could be disconnected from the wiring to facilitate movement along the rails.

The motor integrated with the track actuator did not come equipped with an encoder and therefore the string potentiometer described earlier was used to determine the lateral position. One of the string potentiometer output calibrations was shown above in Figure 3.30. but the calibrated distance from the potentiometer had to be related to the lateral position of the hot-wire probe at the end of the probe support. A calibration curve was developed and is shown in Figure 3.33(a) that relates the string potentiometer distance to 


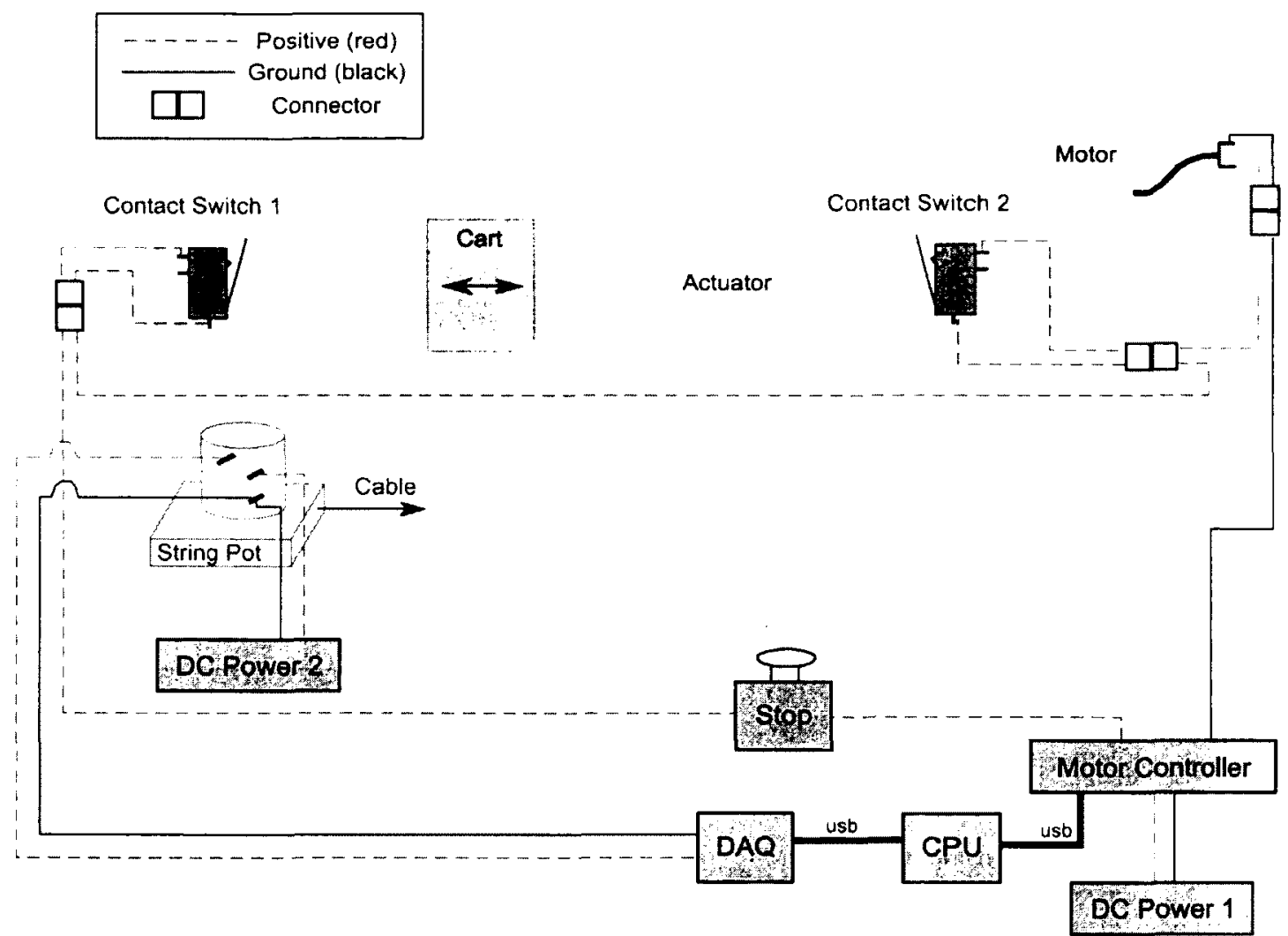

Figure 3.32: Wiring diagram for the traverse system.

the lateral position of the probe from the port side wall of the tunnel. A calibration curve was developed in a similar manner to relate the reading from the Vernier scale $\left(d_{v}\right)$ on the vertical axis to the height of the probe from the test section floor. as shown in Figure $3.33(b)$

\subsubsection{Hot-wire measurements}

The speed in the wake of several turbine configurations was determined in order to complement the turbine power measurements. The sensor that was available to meet this objective was a single-normal hot-wire probe connected to a constant temperature hotwire anemometer. Single-normal hot-wire probes have been used by several authors in Refs. $[41.43,62]$ to characterise the flow downstream of scaled wind turbines. The hot-wire 


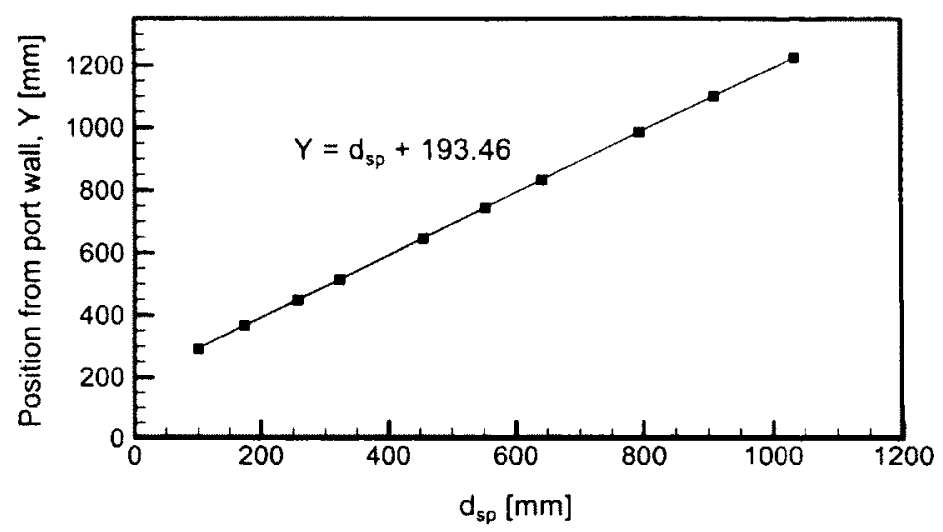

(a) Lateral probe position calibration curve.

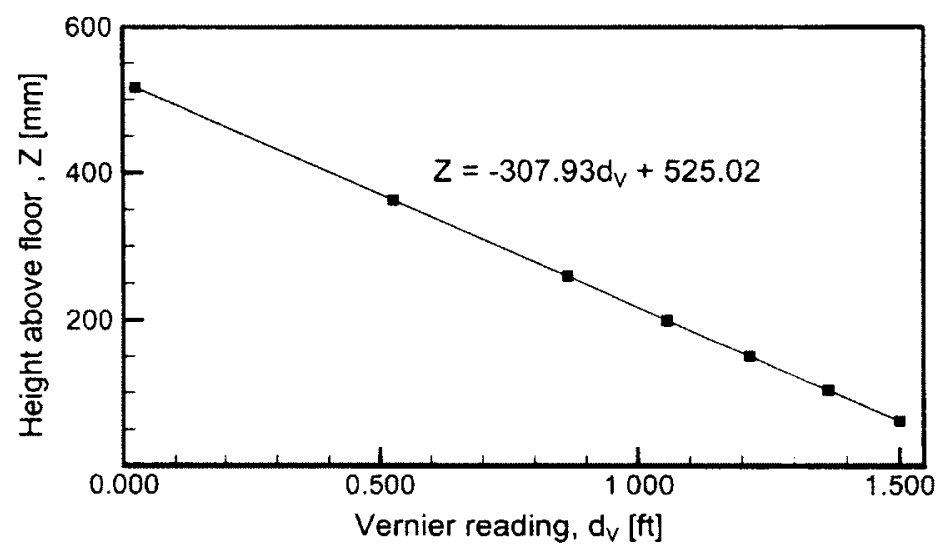

(b) Vertical probe position calibration curve.

Figure 3.33: Calibration curves for determining the position of the hot-wire probe.

probe was manufactured by Auspex Scientific and had a $5 \mu \mathrm{m}$ tungsten welded sensor with a length of $1 \mathrm{~mm}$. The body of the probe was a stainless steel tube with a $1 / 8$ in diameter.

The probe was connected to an AN-1005 constant temperature anemometry system from A.A. Lab Systems Ltd. The anemometer had a single channel. which was equipped with a signal conditioning unit in order to amplify. offset. and filter the signal [161]. The manufacturer-provided guidelines for setting up the anemometer were followed and the parameters in Table 3.5 were applied to the anemometer. The DC offset and the gain were selected in order to match the range of expected flow conditions to the voltage output of the anemometer. This led to a voltage output at zero velocity of approximately $-9.6 \mathrm{~V}$ and a voltage output at the upper range of the measurements of approximately $7 \mathrm{~V}$. Based on 
the results observed in other wind turbine experiments with a similar rotor diameter. the frequencies of interest were typically below $1 \mathrm{kHz}$ [16.64]. Data were subsequently low-pass filtered at a slightly higher frequency. $1.4 \mathrm{kHz}$, and were sampled at twice that frequency. The DAQ receiving the anemometer amplifier output signal had 16-bit resolution. which corresponds to 65.536 discrete values between $-10 \mathrm{~V}$ and $10 \mathrm{~V}$ and discrete voltage intervals of $0.0003 \mathrm{~V}$.

Table 3.5: Anemometer settings.

\begin{tabular}{|c|c|}
\hline Parameter & Value \\
\hline \hline DC Offset & $-3.510 \mathrm{~V}$ \\
\hline Gain & 13.18 \\
\hline Overheat Ratio & 1.488 \\
\hline Filter & $1.4 \mathrm{kHz}$ \\
\hline Sampling Rate & $2.8 \mathrm{kHz}$ \\
\hline Sampling Time & $30 \mathrm{~s}$ \\
\hline
\end{tabular}

\subsubsection{Calibrations and temperature correction}

The hot-wire probe was calibrated against a Pitot-static tube in situ in the downstream test section of the $\mathrm{ABL}$ tunnel. The wind tunnel configuration was modified from the configuration used to generate a scaled ABL, however. The spires and the two upstream roughness panels were removed. This was done in order to limit the growth of the boundary layer so that a core of uniform flow was present at the height where calibration took place. The centre of the Pitot-static probe and the hot-wire probe were located $500 \mathrm{~mm}$ above the floor of the test section and were separated laterally by $35 \mathrm{~mm}$. as indicated in Figure 3.34. A separate study investigated the effect of the interference between the two probes and determined that the probes did not mutually-interfere at this distance.

Hot-wire probe calibrations were conducted throughout the measurement campaign with calibrations conducted before or after experiments and at different ambient temperatures. In total, 17 calibrations were conducted throughout the measurement campaign. Calibration 


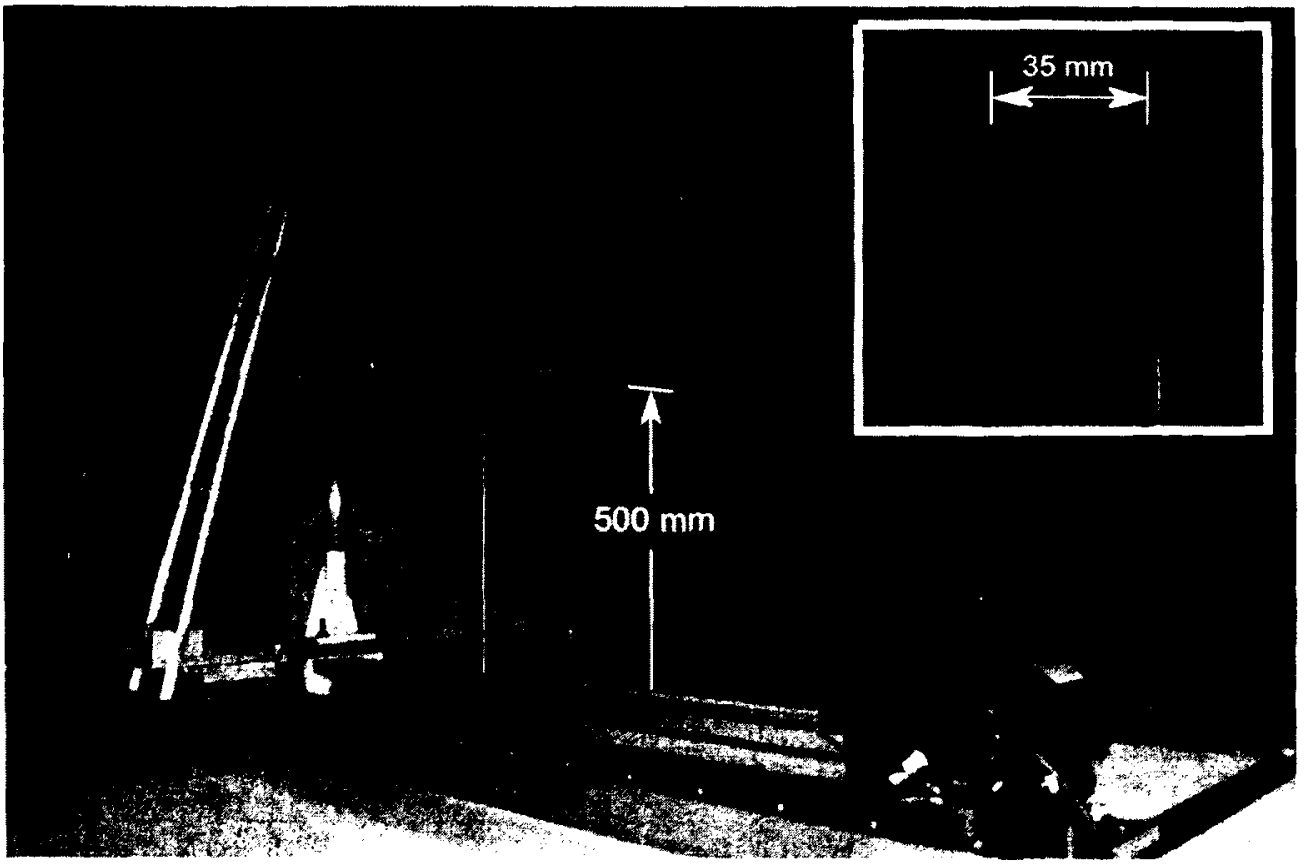

Figure 3.34: Calibration setup in the wind tunnel.

curves are typically presented in literature where the anemometer amplifier output voltage is a function of the speed. An alternate approach is described by Bruun in which the speed is expressed as a fourth order polynomial as a function of the anemometer voltage [162]. The approach highlighted by Bruun simplifies the data reduction procedure and was adopted in the current work. The calibration curves are presented in a manner that follows the calibration equations and therefore the output voltage will be presented on the $\mathrm{x}$-axis and the recorded wind speed will be on the $y$-axis.

A particular challenge that had to be addressed during the calibration procedure related to the minimum freestream speed in the tunnel. The wind speed in the tunnel when the motor was operating at its idle condition was approximately $4.2 \mathrm{~m} / \mathrm{s}$ and this was higher than some of the flow speeds expected in the wakes of downstream turbines. A convenient and suitable low-speed calibration facility in which flow speeds could range from $1-4 \mathrm{~m} / \mathrm{s}$ could not be located and so another solution was explored. A series of sandwiched screens were placed upstream of the hot-wire and Pitot-static probes in order 
to slow down the incoming fluid. The speed of the flow reaching the probes was adjusted by removing individual screens. This approach allowed the flow to be slowed down to 2 $\mathrm{m} / \mathrm{s}$. One of the calibration curves developed during the measurement campaign can be seen in Figure 3.35(a). where the symbols representing the data recorded in the low speed configuration (with screens) are distinguished from those representing the data in the high speed configuration (no screens). Labels corresponding to the number of screens used for certain data points in the low speed data series are identified in Figure 3.35(a). A 4th order polynomial calibration equation was generated using all of the data from the calibration and the percent difference between the data points and the calibration curve is highlighted in Figure 3.35 (b). The errors in the high speed configuration were all within $\pm 0.25 \%$ and those observed in the low speed configuration were typically within $\pm 1 \%$. The majority of the recorded data corresponded to wind speeds above $4 \mathrm{~m} / \mathrm{s}$ and therefore the errors associated with the low speed configuration were deemed to be acceptable. It is worth noting that the data point recorded at the tunnel idle condition without screens often exhibited a larger error than the remaining data obtained in the high speed configuration.

The sensitivity of the calibration curves to changes in ambient temperature was identified using calibration curves developed at the three temperatures indicated in Figure 3.36(a). The temperature in the room was maintained within $\pm 0.1^{\circ} \mathrm{C}$ during each of the calibrations. The results are consistent with calibrations observed in literature in that the same voltage represents a higher speed as the temperature in the room increases. The three calibration curves identified in Figure 3.36(a) were used to interpolate for the wind speed based on the temperature during a particular test. Each experimental run typically lasted $1 \mathrm{hr}$ and the average temperature recorded during the run was used to account for temperature effects. The calibration curves were extrapolated in cases where the temperature exceeded $23.0^{\circ} \mathrm{C}$, the highest temperature associated with a calibration curve. A small number of the experimental runs had velocities above $23.0^{\circ}$ and the highest temperature observed during a 


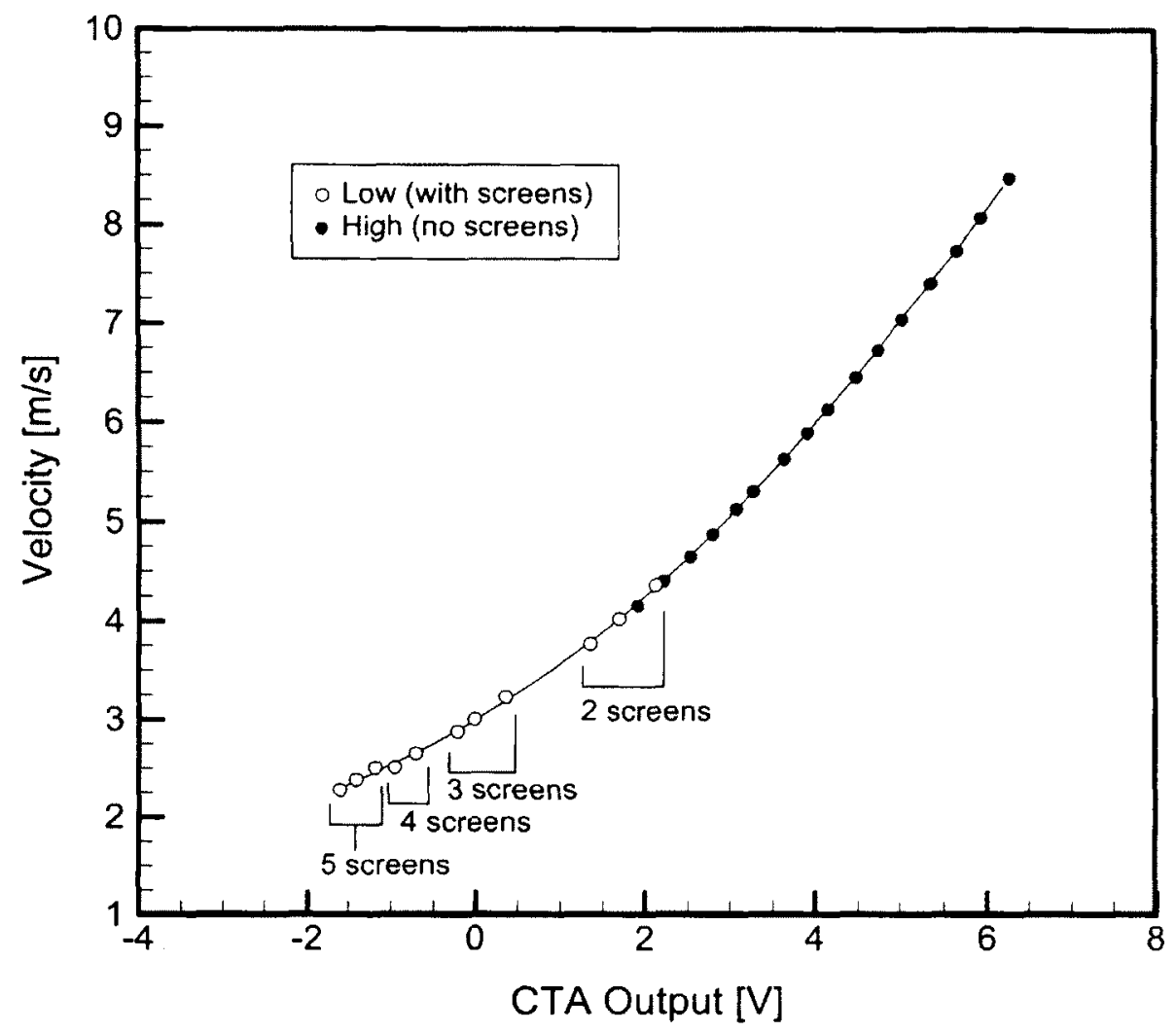

(a) Calibration curve at low and high velocities.

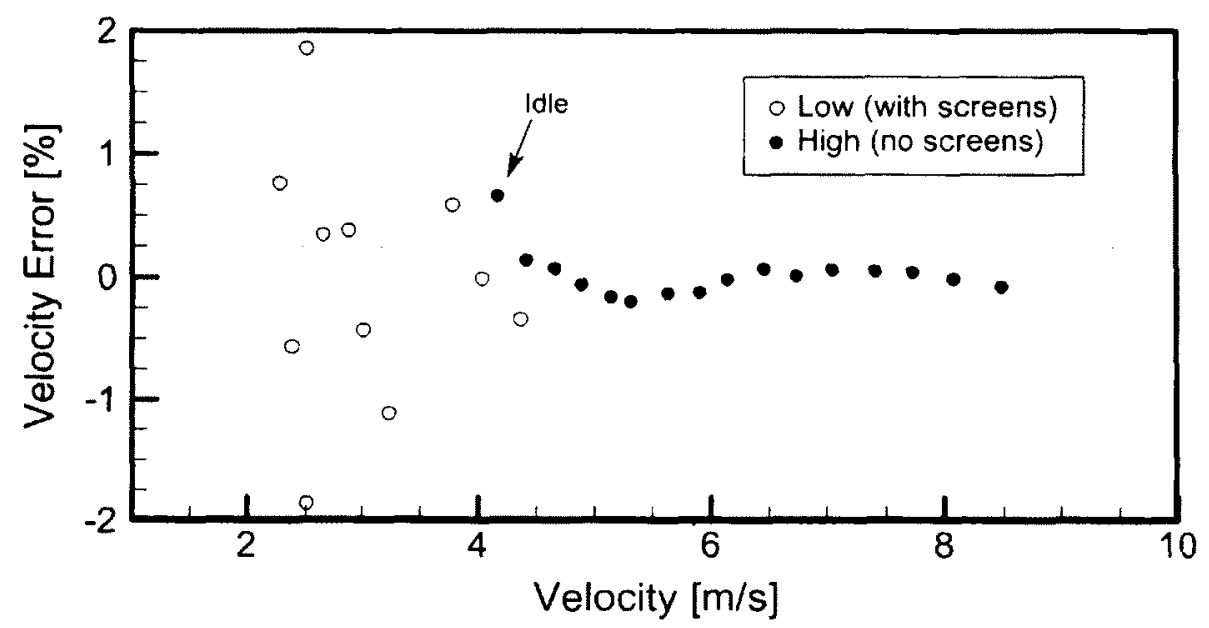

(b) Velocity errors at low and high velocities

Figure 3.35: Calibration curve developed at low and high velocities.

run was $23.7^{\circ} \mathrm{C}$. The errors between the recorded data points and the 4 th order polynomial curve fit are shown in Figure 3.36(b). Similar to the calibration presented earlier. the errors 
associated with the high speed calibration configuration were within $\pm 0.25 \%$ and these errors increased to approximately $\pm 1 \%$ in the low speed configuration.

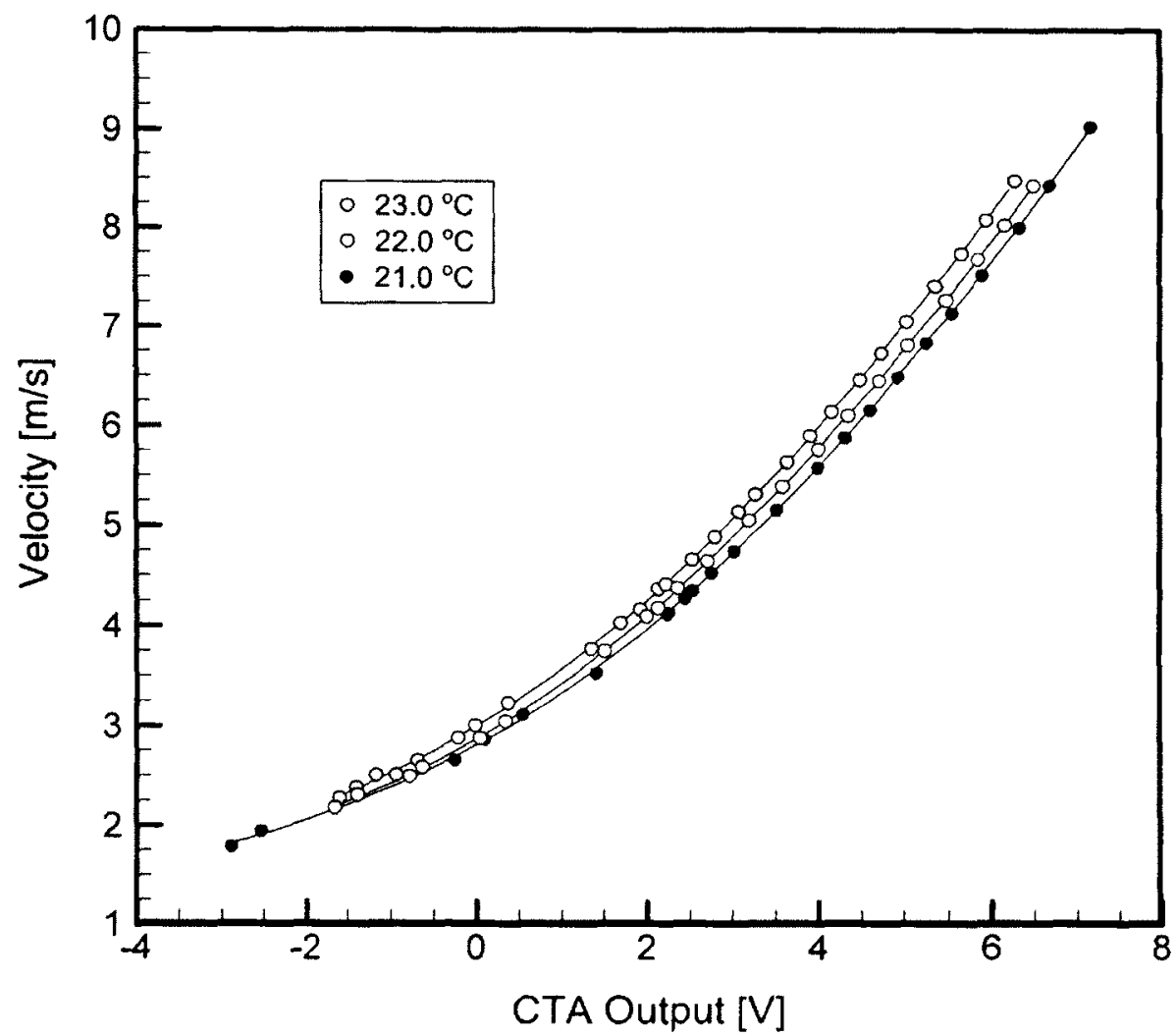

(a) Temperature sensitivity in the calibrations.

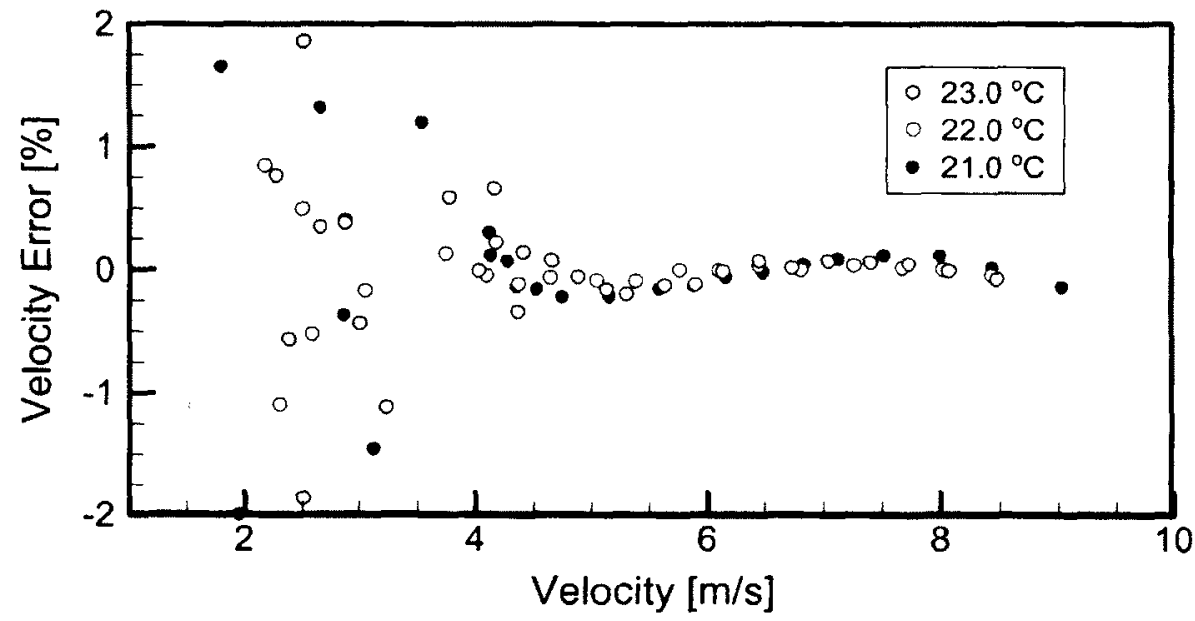

(b) Velocity calibration errors at three temperatures

Figure 3.36: Sensitivity of the calibration curve to changes in temperature. 
Lastly, the long term calibration stability was demonstrated using two calibration curves recorded at the same ambient temperature. The calibration curves presented in Figure 3.37 were recorded 20 days apart and approximately 50 hours of tunnel time was recorded between the two calibrations.

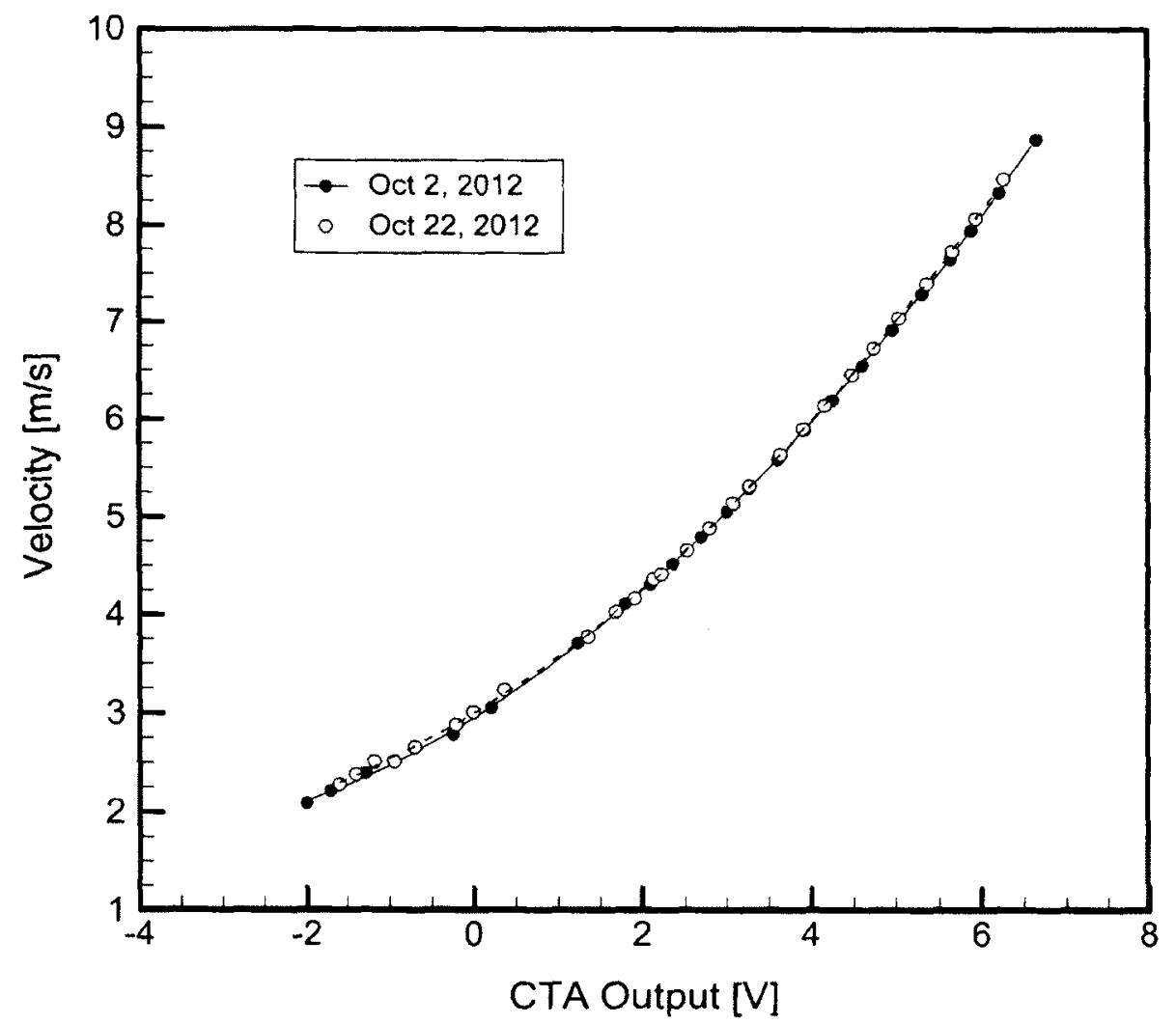

Figure 3.37: Long term calibration stability at $23.0^{\circ} \mathrm{C}$.

\subsubsection{Data reduction}

The speed time series $(U)$ was calculated based on the anemometer amplifier output ( $V_{\text {amp }}$ ) according to Equation (3.31). The calibration coefficients $A$ to $E$ are presented in Table 3.6.

$$
U=A V_{a m p}^{4}+B V_{a m p}^{3}+C V_{a m p}^{2}+D V_{a m p}+E
$$

The mean speed was calculated for each 30 second sample. The turbulence intensity 
Table 3.6: Calibration coefficients.

\begin{tabular}{|c|c|c|c|c|c|c|}
\hline Calibration & Temp & $\mathrm{A}$ & $\mathrm{B}$ & $\mathrm{C}$ & $\mathrm{D}$ & $\mathrm{E}$ \\
\hline \hline 10 & $21.0^{\circ} \mathrm{C}$ & -0.0002 & 0.0019 & 0.0505 & 0.4682 & 2.8103 \\
\hline 12 & $22.0^{\circ} \mathrm{C}$ & 0 & -0.0006 & 0.0566 & 0.5017 & 2.8692 \\
\hline 17 & $23.0^{\circ} \mathrm{C}$ & -0.0002 & 0.0022 & 0.0511 & 0.5151 & 3.0000 \\
\hline
\end{tabular}

(Tu) was calculated using Equations (3.32) and (3.33). where $U_{r m s}$ is the root-mean-square of the speed and $U_{r e f}$ is the mean reference speed. $U_{r e f}$ corresponds to the mean speed at hub height in the test section without wind turbines following the results presented in Refs. [16.43,64.79] as opposed to the mean local speed at a particular data point.

$$
\begin{gathered}
U_{r m s}=\left(\frac{1}{N-1} \sum_{1}^{N}\left(U_{i}-U_{\text {mean }}\right)^{2}\right)^{0.5} \\
T u=\frac{U_{r m s}}{U_{r e f}}
\end{gathered}
$$

\subsection{Computational methods}

The two computational methods used during the thesis research were blade element momentum theory (BEMT) and a vortex particle code. The BEMT was previouslydiscussed in Section 3.1 and as the related theory is well explained in literature. the method will not be described in the thesis [86-88]. The vortex particle code, GENUVP, was used to support a portion of the research and offers the ability to evaluate the development of the wake in freestream conditions. 


\subsubsection{GENUVP}

GENUVP is an inviscid. incompressible. discrete vortex method computational solver that was developed at the National Technical University of Athens and the software has been used to characterise a number of helicopter and wind turbine problems [118,163]. A complete treatment of the theory and implementation of the code can be found in Refs. [110.112]. The simulation domain in GENUVP is divided into three regions: the rotor blade surface. the near wake region, and the far wake region. Singularity distributions are defined on all solid surfaces and in the near wake, whereas the farwake behind the lifting surface is represented with free vortex particles [112]. The rotor blades are resolved using thin lifting surfaces and an inviscid panel method is used to resolve the blade forces. A viscous correction is implemented using lookup tables with two-dimensional lift and drag airfoil data.

At each time step. a vortex sheet is generated along the trailing edge of a blade and at the blade tip. This vortex sheet is then transformed into free spatial vorticity in subsequent steps so that the vorticity in the far wake may be represented by free vortex particles. Therefore, instead of calculating the geometry of vortex sheets in the far wake, the evolution of free vortex particles is tracked throughout the domain [110.112].

A computational simulation of the scaled MAAE rotor was conducted using GENLVP as part of the blockage study described in Chapter 4 in order to identify tip vortex positions in freestream conditions. The computation was performed in freestream conditions with a rotor operating at $\lambda=6$ and $R e_{t i p}=24.000$. A rigid body simulation was conducted as the radial structural properties of the blade were not known. Two-dimensional E387 airfoil lift and drag coefficients from Refs. [149-151] were used to correct the panel method predictions for viscous effects. The viscous correction in GENUVP takes the form of a lookup table populated with steady 2D experimental data at several Reynolds numbers and angles of attack. Experimental airfoil data for the E387 airfoil at Reynolds numbers ranging from 
10.000 to 30.000 were used. The reduction in the lift coefficient due to the $2 \mathrm{D}$ experimental data correction ranged from $25 \%$ at the mid-span to $17.5 \%$ at the blade tip. The magnitude of the correction is due to the shallow slope of the lift curve of the E387 airfoil when the Reynolds number is less than 50.000 .

The inboard section of the blades from the spanwise location $r / R=0.1$ to $r / R=0.3$ has a local Reynolds number below 10,000 when $R e_{t i p}=24.000$. It was estimated that the inboard $20 \%$ of the current rotor blades only generates approximately $8 \%$ of the total rotor torque and, as a result, it was assumed that the airfoil data at this inboard section could be approximated with the available data at a Reynolds number of 10,000 [151].

\subsubsection{Validation}

GENUVP will be used to assess the wake development downstream of the three-bladed MAAE rotor in freestream conditions in the next Chapter. It was therefore desired to validate the ability of the vortex particle code to capture the expansion of the wake in the near wake region. The force and torque predictions from GENUVP have been previouslyvalidated by this author using data from the NREL Phase VI experiment in 2008 in Ref. [20]. The two-bladed $10 \mathrm{~m}$ diameter wind turbine is seen below in Figure 3.38 in the NASA Ames $24 \mathrm{~m} \times 36 \mathrm{~m}$ wind tunnel. The stall-controlled wind turbine has twisted, tapered blades that have an S809 airfoil and a tip pitch angle of $3^{\circ}$. The experiments conducted by the NREL operated the turbine with a fixed rotational speed of $72 \mathrm{rpm}$ and wind speeds that varied from $5 \mathrm{~m} / \mathrm{s}$ to $25 \mathrm{~m} / \mathrm{s}$. Qualitative smoke visualisation was conducted during the full-scale measurement campaign in order to observe one of the helical tip vortices. but the wake expansion was not quantified. Fletcher et al. conducted CFD simulations of the NREL rotor using RANS and vorticity transport method (VTM) approaches in 2009 [120]. Experimental data wasn't available for comparison until recently in 2011. however. when 
a medium-scale experiment was conducted by Xiao et al. with a $1 / 8$ scale model of the NREL turbine [50]. Given that the NREL Phase VI turbine had been used for previous validation efforts. it was decided to validate the wake profile predicted by GENUVP with those predicted in the computational work of Fletcher et al. and in the experimental work of Xiao et al. $[50,120]$.

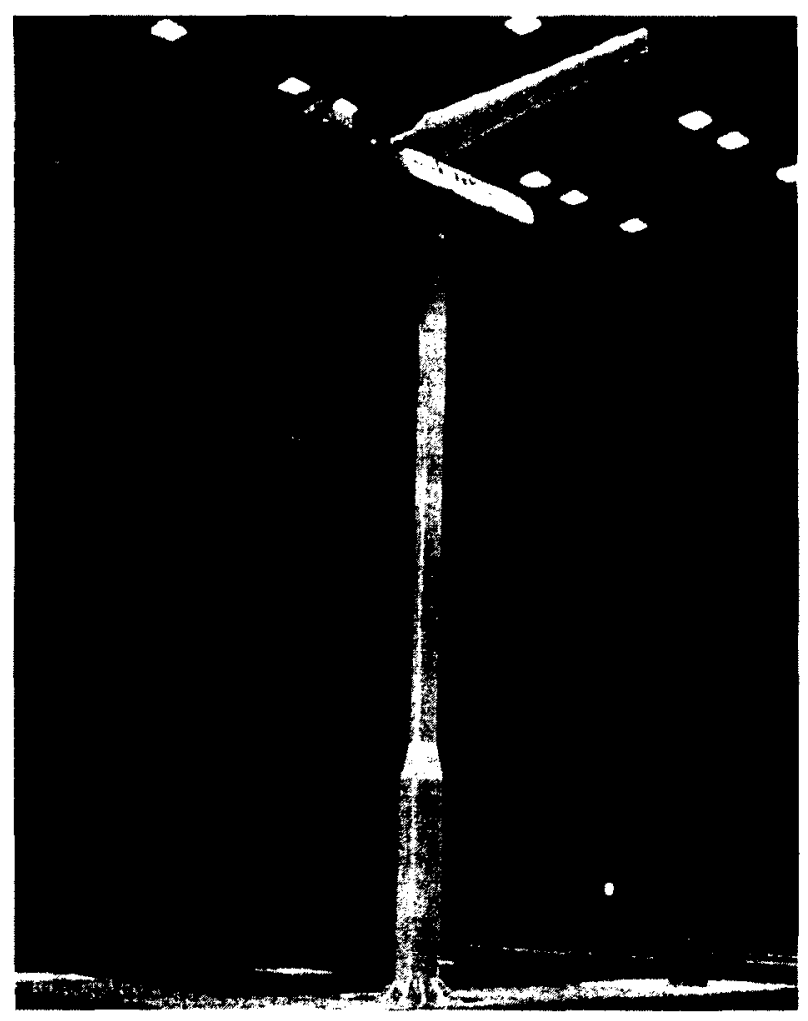

Figure 3.38: The NREL Phase VI UAE turbine [56].

Xiao et al. recorded PIV measurements of the tip vortex positions downstream of the blade throughout a wake age of $450^{\circ}$, corresponding to approximate 1.3 revolutions of the turbine [50]. The axial and lateral positions of the tip vortex cores were identified at nonuniform wake age intervals. The rotor was tested in flow conditions that produced $\lambda=4.9$ and $R e_{t i p}=175.000$. These conditions are compared to the test conditions used in the computations by Fletcher et al. and in the current work in Table 3.7. The tip speed ratio in the computations is $10 \%$ higher than that used in the experiment. The lower tip speed ratio 
in the experiment should generate a wake with less lateral expansion and a larger helical tip vortex pitch distance.

Table 3.7: Comparison between the computational and the experimental test conditions from Ref. [50].

\begin{tabular}{|c|c|c|c|}
\hline Description & Diameter & $\lambda$ & $R e_{\text {tip }}$ \\
\hline \hline Present work - GENUVP & $10 \mathrm{~m}$ & 5.4 & 900.000 \\
\hline Fletcher et al. $[120]$ - RANS & $10 \mathrm{~m}$ & 5.4 & 900.000 \\
\hline Fletcher et al. $[120]$ - VTM & $10 \mathrm{~m}$ & 5.4 & 900,000 \\
\hline Xiao et al. $[50]$ - PIV & $1.25 \mathrm{~m}$ & 4.9 & 175,000 \\
\hline
\end{tabular}

A schematic highlighting the computational flowfields and the coordinate system for the validation case is presented in Figure 3.39. The tip vortices generated in the RANS computations from Ref. [120] dissipate after 1.5 turbine revolutions and the wake profile is qualitatively similar to the flowfield highlighted by the vortex particles from GENUVP. The wake age from $0^{\circ}$ to $540^{\circ}$ is represented schematically in Figure 3.39, along with the conventions used for the downstream and lateral tip vortex positions.

The current computational results are compared to the results from Fletcher et al. and Xiao et al. in Figure 3.40. The downstream and lateral tip vortex core locations were identified in $20^{\circ}$ intervals in the computations and an irregular distribution of data points was recorded in the computations. The tip vortex age is limited to $540^{\circ}$ of rotation in Figure 3.40 as this encompasses the available range of data for both the experimental results of Xiao et al. and the RANS simulation of Fletcher et al. [120].

Good agreement can be seen when comparing the downstream tip vortex position as a function of wake age in Figure 3.40(a). The increased axial distance at a particular wake age in the experiment is consistent with the reduced $\lambda$ in that case and corresponds to a helical vortex with a larger pitch.

The four data sets compared in Figure 3.40(b) highlight a number of similar features. In all cases, the wake appears to contract near the blade tip due to tip vortex roll-up prior 


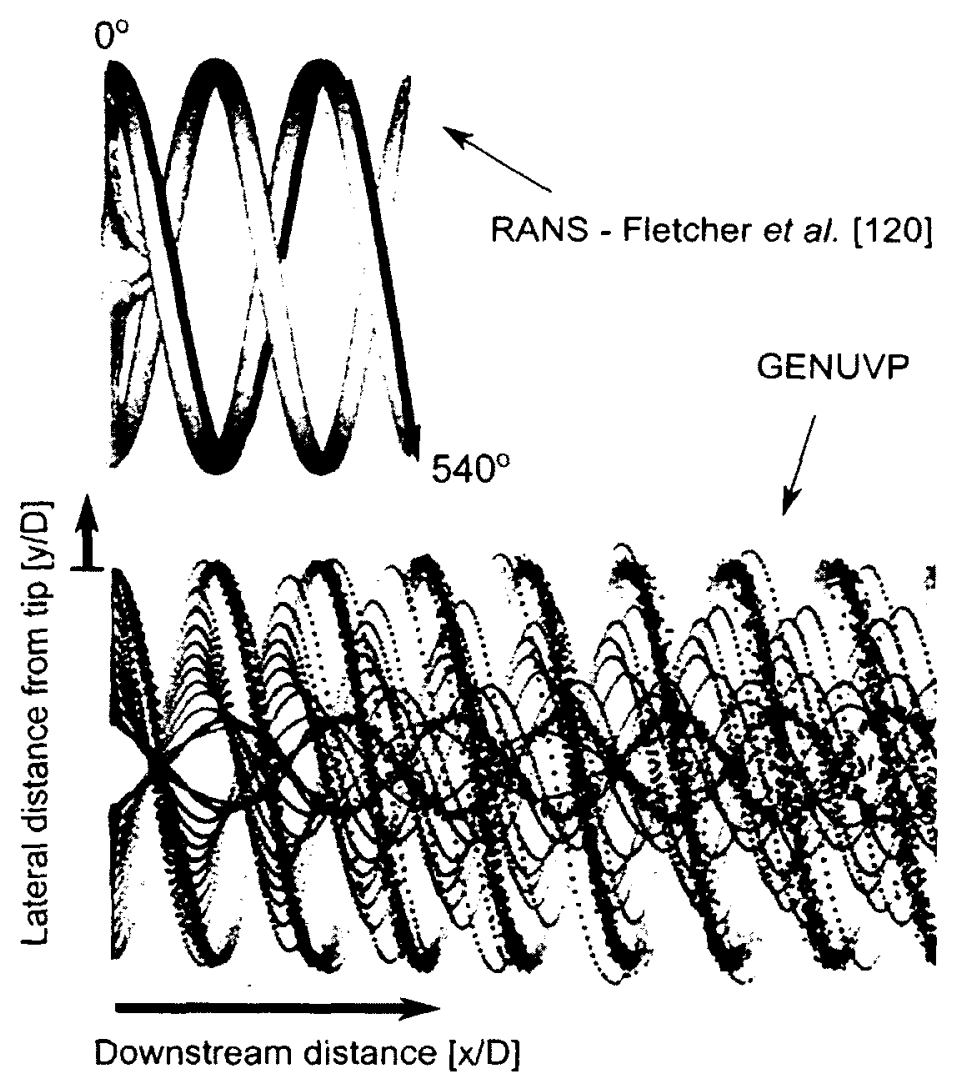

Figure 3.39: Comparison of the computational wake profiles.

to expanding further downstream. The small expansion of approximately $1 \%$ is attributed to the low solidity and low thrust coefficient of this wind turbine $\left(C_{T} \approx 0.49\right.$ at $\lambda=$ 5.4). The current results obtained with GENUVP are well validated and closely follow the lateral vortex position from the RANS simulations. The prediction of the wake expansion downstream of the MAAE rotor will be presented in Chapter 4. 

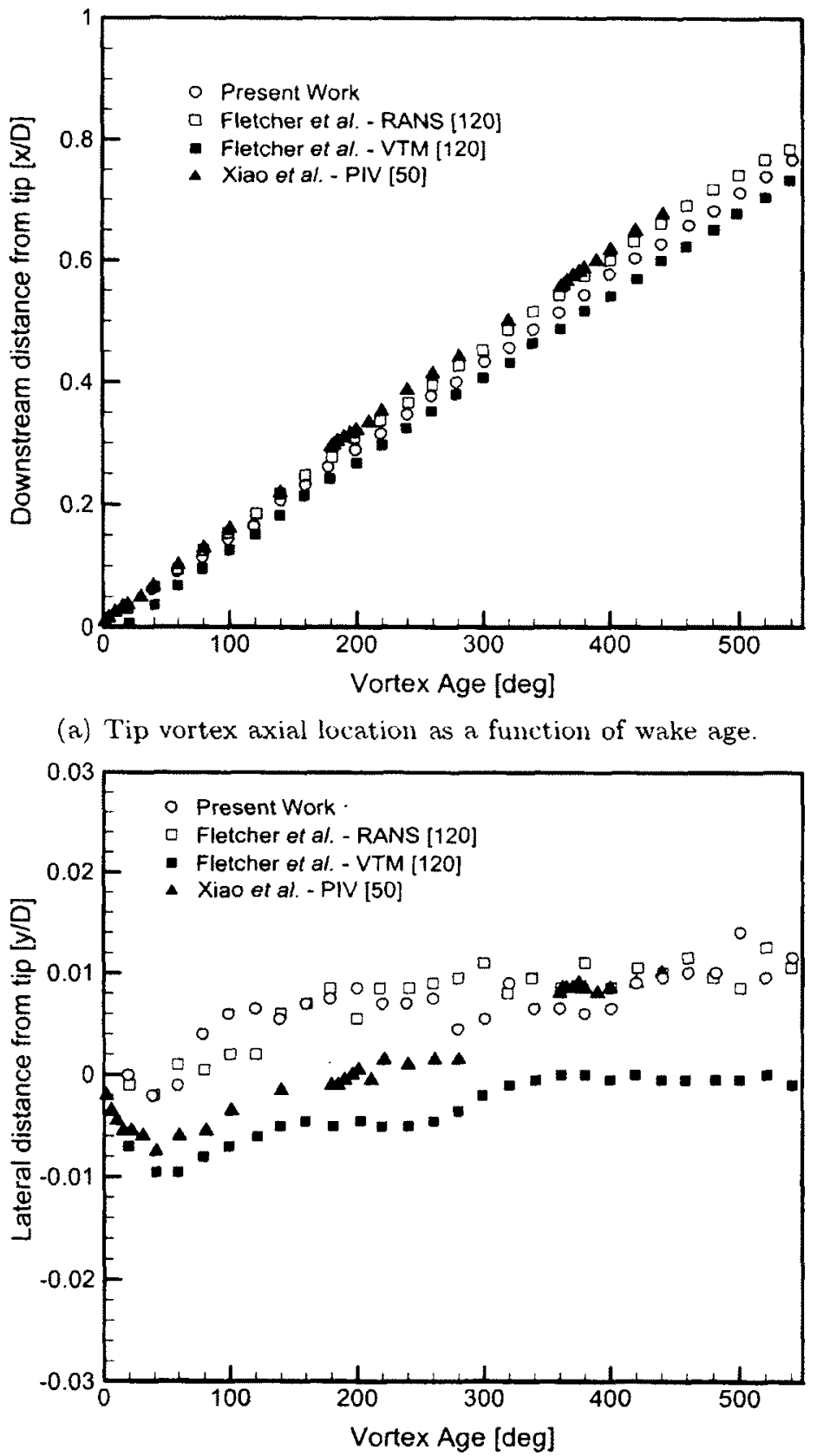

(b) Tip vortex radial location as a function of wake age.

Figure 3.40: Validation of the axial and radial tip vortex locations. 


\section{Chapter 4}

\section{Evaluating blockage effects on}

\section{wind turbine wake development}

One concern in scaled wind turbine experiments is the extent of the blockage effects on the wake development, as the factors influencing wake expansion in wake interference experiments should be limited to the surface representing the ground. neighbouring wakes. the turbulent inflow conditions, and atmospheric stability. The objective of the current study was to evaluate the effect of blockage on the initial wake expansion downstream of a scaled wind turbine in a closed test section. The initial wake expansion was assessed by identifying the tip vortex cores downstream of the five rotors using quantitative dye visualisation. The thrust developed by the scaled models was recorded using a six degreeof-freedom balance and has been related to the wake expansion.

This Chapter will first present a series of tests that were completed in order to assess the behaviour of the dye visualisation and force measurement systems. The flowfield observed downstream of the scaled wind turbines will be described using experimental and computational results. The blockage results in the near wake will then be presented and 
will be compared to the wake expansion determined computationally using a discrete vortex method. Lastly. a relationship between the wake profile and the recorded thrust coefficient will be established. The results of the study will help to develop testing guidelines that minimize the effect of the test section on the natural development of the flow downstream of a rotor.

\subsection{Assessment of the experimental setup}

The measurement campaign described in this Chapter represents the first quantitative data set obtained using the RHRC 2436 water channel. It was therefore desired to develop a series of recommended practices by thoroughly evaluating the experimental setup.

The RHRC 2436 water channel was previously described in Chapter 3 and is equipped with a 6-colour dye visualisation system. The dye was composed of food colouring from Esco Foods, Inc., that was mixed in proportions of 3 parts water and 1 part food colouring. in order to produce dye that was close to neutrally-buoyant. Although two of the dyecontaining canisters were broken during the test campaign, red, green, blue, and brown dyes were all tested. The brown dye provided the sharpest contrast in the recorded photographs. The blue dye produced acceptable contrast. but the flow patterns highlighted by the red and green dyes were more difficult to clearly distinguish in the water. With the exception of two tests, all of the remaining flow visualisation tests used brown dye.

The dye visualisation system represents an upstream obstruction in the test section and the system was designed to minimise the effects of the dye strut and dye tube on the flow behaviour. The dye strut was a streamlined rod and the dye tube was a slender. tapered tube. The outlet of the dye tube was located more than 8 strut-chord widths downstream of the dye strut in order to limit the effect of the wake shed from the dye strut on the 
injected dye. The frontal area of the assembly represented $0.5 \%$ of the test section area and its effect on the overall level of blockage in the channel was neglected. While the blockage contribution of the strut and tube were neglected. several aspects of the dye system were characterised during the flow visualisation campaign. including:

- the effect of the dye setting on the initial wake expansion.

- the effect of the dye setting on the measured forces.

- the effect of isolating the turbine strut from the wake.

\subsubsection{Effect of the dye setting on the initial wake expansion}

The primary means of assessing the effect of blockage on wake development in this thesis is through the use of quantitative dye visualisation to identify tip vortex core locations. The first testing parameter to be evaluated was therefore the effect that the dye flow rate. or dye setting, had on the wake expansion.

The effect of the dye setting on the wake expansion was evaluated using a $25 \mathrm{~cm}$ rotor, corresponding to $10 \%$ blockage at a Reynolds number of 9.370 and a tip speed ratio of approximately 6 . Recall from Chapter 3 that images were digitally-processed and the extracted tip vortex coordinate locations relative to the blade tip were subsequently corrected for parallax and were normalised with respect to the turbine diameter. Four dye settings were tested and five flow visualisation images were processed at each dye setting. The initial wake expansion was subsequently calculated using the parallax-corrected vortex cores extracted from the images. All of the images were recorded when Blade 2 was passing through the dye stream, as previously discussed. The flowfield visualised at the four tested dye settings can be seen in Figure 4.1. A smooth dye stream can be seen upstream of the rotor blade at the low. medium, and high dye settings. However. the very high dye setting in 
Figure 4.1(d) creates a mushrooming effect upstream of the rotor blade as the dye behaves like an impinging jet in the freestream flow.

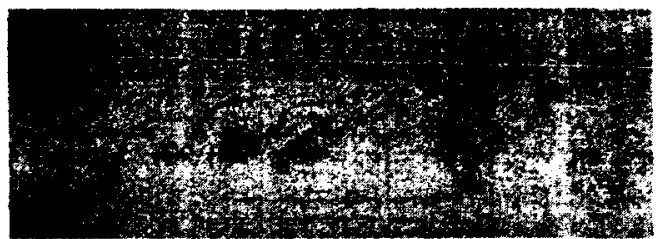

(a) Low dye setting.

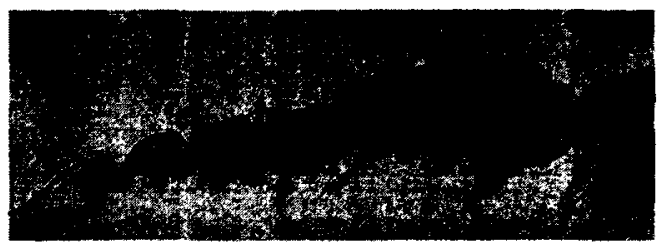

(c) High dye setting

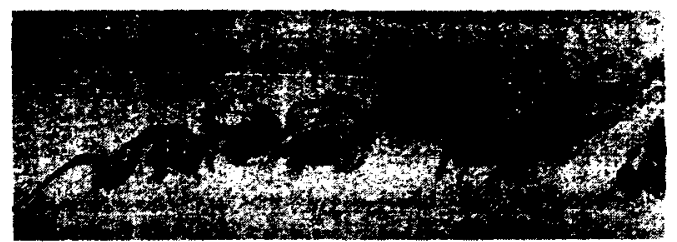

(b) Medium dye setting.

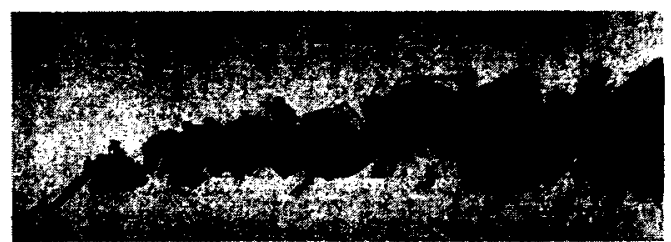

(d) Very High dye setting.

Figure 4.1: Tip vortex visualisation at different dye settings with a $25 \mathrm{~cm}$ rotor at $\lambda \approx 6$ and $R e_{t i p}=9.370$.

The position of the tip vortices in the wake calculated based on images similar to those seen in Figures 4.1(a) to 4.1(d) is presented in Figure 4.2. Figure 4.2 demonstrates that the initial wake expansion profile experienced small variations when the low. medium. and high dye settings are considered. The very high dye setting appears to increase the observed wake expansion, however. The error bars visible in Figure 4.2 represent \pm 1 standard error from the mean vortex core locations. Although the horizontal and vertical error bars have been displayed, in many cases the horizontal error bars were smaller than the symbol size. The vertical error bars represent a standard error of less than $\pm 2 \%$ to $\pm 5 \%$.

The effect of the dye on the tip vortex core location was further assessed by calculating the percent difference between each radial core location relative to the vortex core location with the lowest dye setting. The lowest dye setting was selected as the baseline condition as it was expected that the dye would interfere the least with the flowfield developing downstream. The deviation of the lateral tip vortex core locations with the medium. high. and very high dye settings from the first five core locations identified with the low dye setting 


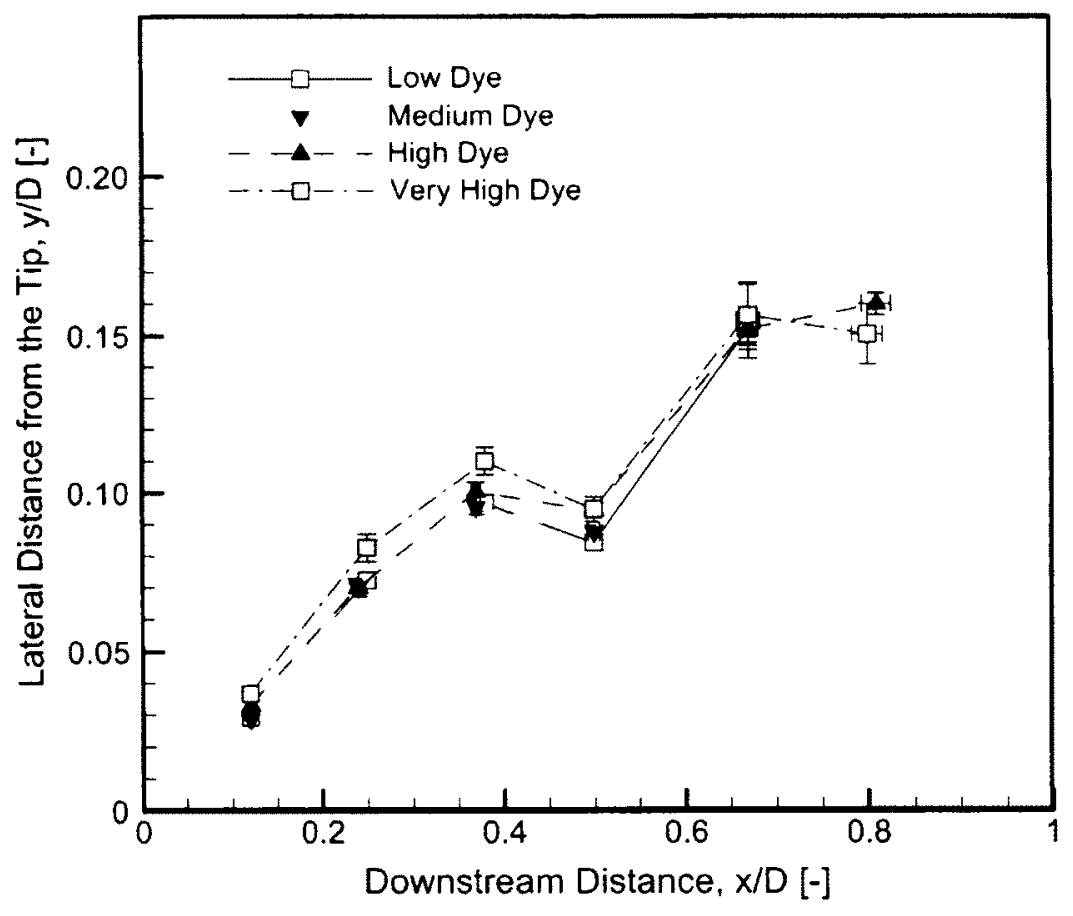

Figure 4.2: Initial wake expansion as a function of the dye setting: $25 \mathrm{~cm}$ rotor at $\lambda \approx 6$ and $R e_{t i p}=9.370$.

can be seen in Figure 4.3. The vortex cores identified with the medium setting typically have small variations compared to the baseline results. The core locations identified with the very high setting, however, consistently have a percent difference larger than $10 \%$ compared to the baseline. This occurs due to the added momentum of the dye that amplifies any potential dye tube misalignment.

Based on these observations, the dye setting in the remaining experiments was maintained near the medium level. A first-order method of obtaining this condition is to ensure that the dye stream appears smooth upstream of the rotor blade so that the jet-like effect visible in Figure 4.1(d) is not visible in the water. This has an additional practical advantage as the water takes longer to become discoloured with a lower dye setting. 


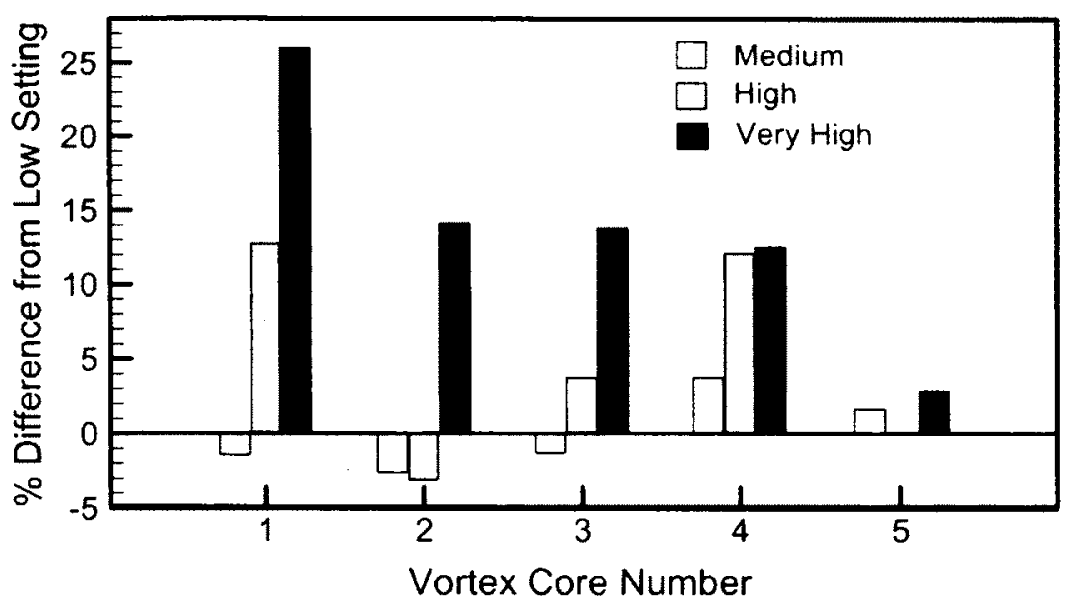

Figure 4.3: Deviation of the lateral vortex core locations due to the dye setting.

\subsubsection{Effect of the dye setting on the measured forces}

The force measurements were recorded in separate measurement campaigns from the dye visualisation experiments. This was done primarily in order to limit the blockage in the channel to the rotor and its support strut but also to minimize the amount of time that the six degree-of-freedom balance spent near the surface of the water. In order to assess whether or not future campaigns could collect flow visualisation and force data simultaneously. the forces generated by a rotor were recorded at various dye settings. The change in the uncorrected thrust coefficient $\left(C_{T, U}\right)$ as a result of the dye setting was evaluated using two separate runs. The procedure involved starting the tunnel and allowing the transients to dissipate. The wind turbine forces were recorded without the dye turned on. and then the dye was turned on and the flow rate was increased in discrete intervals approximately every 90 seconds.

The thrust coefficient calculated based on the recorded forces during Run A and Run $\mathrm{B}$ at all of the dye settings is presented in Figure 4.4. The errors that were assessed in the thrust coefficient are included in Figure 4.4 and represent approximately $0.16 \%$ of the thrust coefficient magnitude. In both test cases (A \& B). the thrust coefficient is observed 
to increase with increasing dye flow rate. The change in the measured $C_{T, U}$ is small. however. In Run A. the low dye setting represents an increase of $0.73 \%$ compared to the no dye setting. The very high dye flow rate results in a thrust coefficient that increases by $2.4 \%$ compared to the baseline case. Based on these observations, thrust readings and dye visualisation results can be obtained simultaneously. Thrust coefficient corrections could be implemented with the lower dye flow rates, but the very high dye setting should be avoided when obtaining quantitative results because of its demonstrated impact on the tip vortex locations.

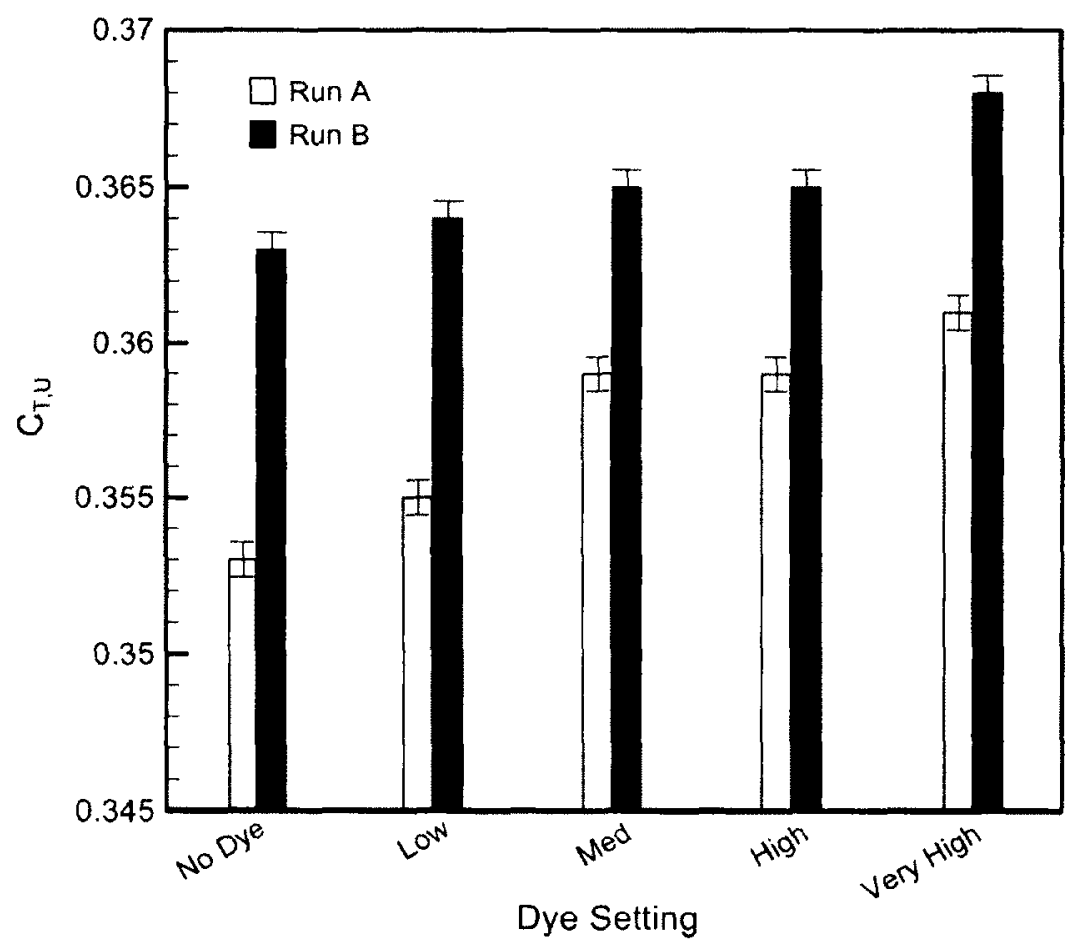

Figure 4.4: Effect of the dye setting on $C_{T, U}: 25 \mathrm{~cm}$ rotor at $\lambda \approx 6$ and $\operatorname{Re}_{\text {tip }}=9.370$.

\subsubsection{Effect of isolating the turbine strut from the wake}

The last aspect of the experimental setup that was considered involved the effect of isolating the vertical turbine support strut from the wake. The wind turbine strut was connected directly to the scale in the force measurement campaigns. As a result, the shed 
tip vortices would strike the support strut and would create a higher perceived thrust force measurement. One practical solution to this problem involves isolating the turbine strut tower from the wake with a concentric pipe. The $0.8 \mathrm{~cm}$ diameter turbine support strut was isolated from the effects of the wake using a $2.2 \mathrm{~cm}$ diameter pipe. The pipe was fixed to the underside of the support platform using a flange and did not touch the support strut.

The effectiveness of this technique is demonstrated by comparing the thrust coefficient recorded with the isolation pipe to the perceived thrust coefficient recorded without the isolation pipe. This was assessed using a $25 \mathrm{~cm}$ rotor at $\lambda \approx 6$ and $R e_{\text {tip }}=9.370$. These results are compared in Figure 4.5, where it can be observed that the test case without the pipe generates a higher recorded thrust coefficient than the case where the strut is isolated using a pipe. In this particular case, when the strut drag (recorded in a separate test) was removed from the forces recorded without the pipe, the results collapse to a similar level as the isolated case. It is expected that at higher flow speeds and Reynolds numbers. the procedure of removing the strut drag will not result in a thrust coefficient that is equivalent to the case where the strut is isolated from the wake due to the added contribution of the shed tip vortices. The concentric pipe was effective, however, in isolating the thrust coefficient developed solely by the rotor.

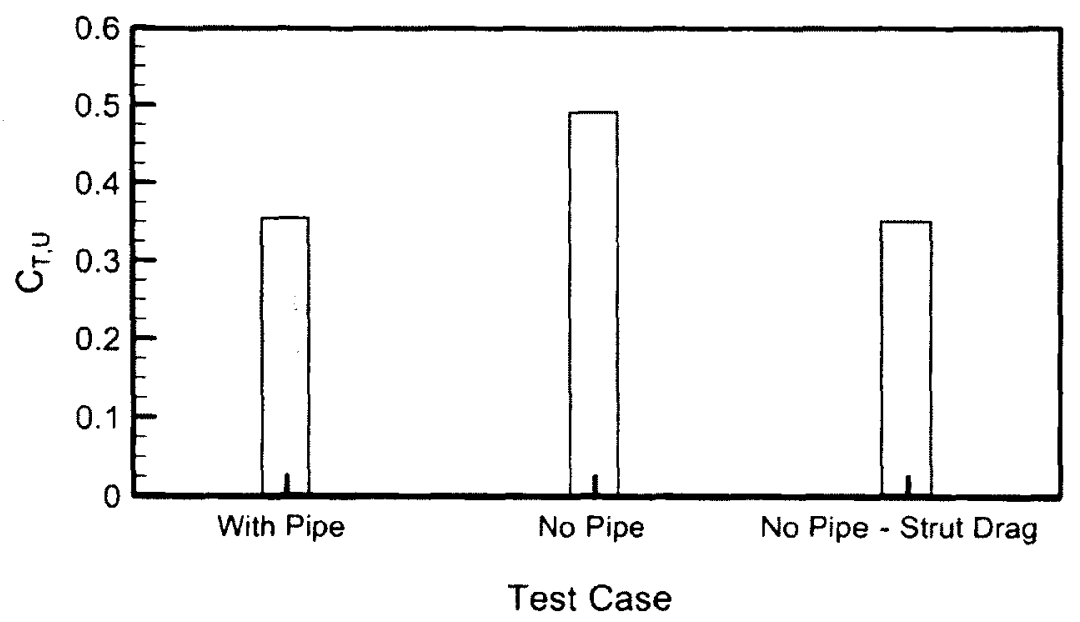

Figure 4.5: Effect of isolating the strut on $C_{T, U}: 25 \mathrm{~cm}$ rotor at $\lambda \approx 6$ and $R e_{\text {tip }}=9.370$. 


\subsubsection{Summary of the developed testing guidelines}

Based on the results obtained in this Section. the following recommendations can be made regarding the dye visualisation and force measurement setup:

- The dye flow rate should be maintained at a low-to-medium level in order to limit the effect of the dye stream on the developing flow structures. The useful range of dye settings corresponds to the labels 11-15 on the dye control dial on the water channel.

- Forces and flow visualisation can be recorded simultaneously, provided the dye setting is maintained in the low-to-medium range. This limits the increase in $C_{T, U}$ due to the dye stream to approximately $1 \%$.

- If the forces are being measured using a support strut attached directly to the turbine and the scale, the strut should be isolated from the turbine wake and the freestream using a concentric pipe. This eliminates the need to remove the strut drag from the recorded forces and removes the effect of the turbine wake on the thrust force.

\subsection{Wake development}

The flowfield in the near wake of the wind turbines was evaluated prior to assessing blockage results in order to understand the flow features that are affected by blockage. Examples of the recorded tip vortex visualisation images are presented in Figure 4.6. These particular images were recorded using a $25 \mathrm{~cm}$ diameter rotor at a Reynolds number of 9,370. Although this $R e_{\text {tip }}$ varies from the blockage test conditions that will be discussed below, the qualitative flow patterns are similar to those observed at $R e_{\text {tip }} \approx 23.000$. The dye diffused more slowly at the lower $R e_{t i p}$. allowing more of the flowfield to be observed. The very high dye setting visible in Figure 4.6 was used to improve the visibility of the 
downstream flowfield, but a lower relative dye setting was used when recording the images used for quantitative dye visualisation based on the testing guidelines that were established earlier in Section 4.1.

The nomenclature in Figure 4.6 is organised such that A. B. C refer to groups of vortices from the first, second, and third revolutions, respectively, and the numbers $1,2.3$ refer to the vortices shed by Blades 1, 2, and 3, respectively. A periodic vortex pairing instability occurred downstream of the rotor and the observed vortex core pattern at a particular instant was dependent on the blade passing through the dye stream at the $0^{\circ}$ azimuth position, as observed in Figures 4.6(a) to 4.6(c). Two sets of three vortices are visible downstream of the rotor in Figure 4.6(a), as indicated by the labels $\mathrm{A}$ and B. Vortices A1 and $A 2$ begin to pair when Blade 1 is in the $0^{\circ}$ azimuth position. When Blade 2 passes through the dye stream as shown in Figure 4.6(b). vortices A1 and A2 continue to pair. while vortex A3 moves outward in the radial direction in the process of coalescing with vortices $A 1$ and A2. Vortex B1 moves inward due to its direction of rotation and the presence of the shear layer between the wake and the freestream and subsequently begins to pair with vortex B2. The first vortex in a new set of vortices. denoted C1. is formed when Blade 2 is in the configuration shown in Figure 4.6(b). When Blade 3 is in the $0^{\circ}$ azimuth position as shown in Figure 4.6(c), the vortices in Group A have paired further while diffusing and growing, while a second vortex in the new set of vortices. denoted C2, is formed. This vortex pairing process is repeated once per rotor revolution at this operating condition. The flow visualisation images used to evaluate the wake expansion were recorded when Blade 2 was in the $0^{\circ}$ azimuth position, corresponding to the flow pattern observed in Figure 4.6(b).

The vortex merging behaviour can be visualised further in Figure 4.7 using an image that has been post-processed. The background has been removed and the visible but diffused dye could be highlighted using the Sobel edge detection algorithm in the image processing software GIMP. The vortices present in Figure 4.7 represent the vortices shed from the 25 


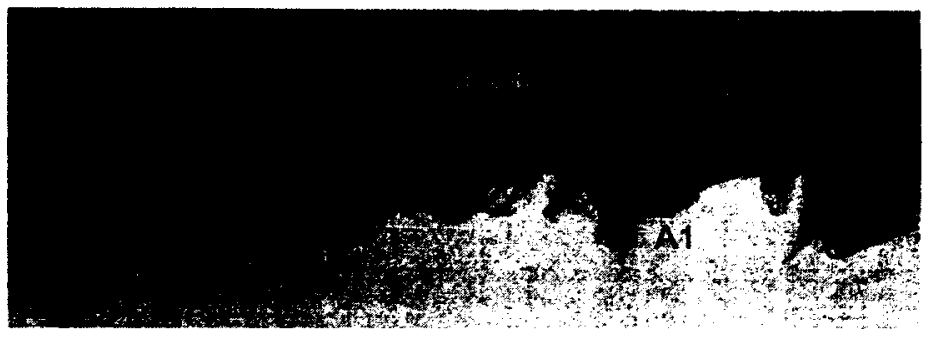

(a) Blade 1 at $0^{\circ}$

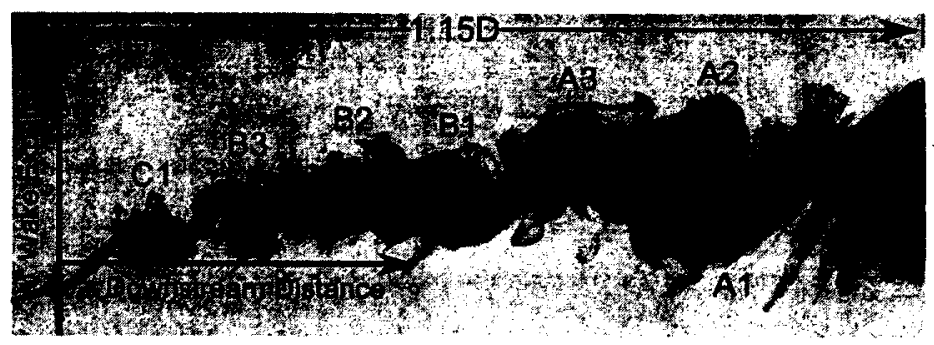

(b) Blade 2 at $0^{\circ}$

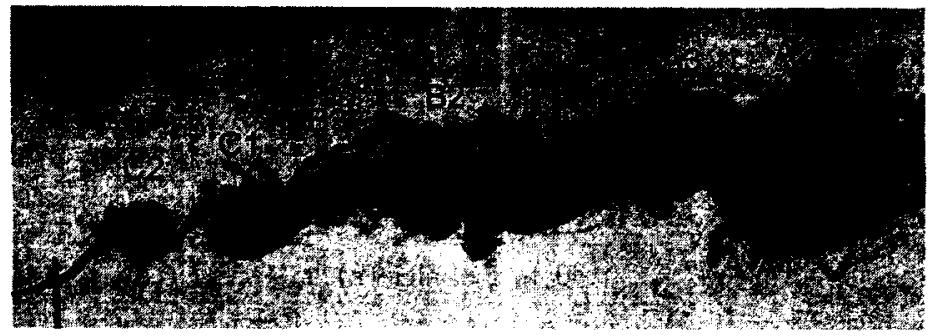

(c) Blade 3 at $0^{\circ}$

Figure 4.6: Tip vortex visualisation downstream of a $25 \mathrm{~cm}$ rotor at $\lambda=6.1$.

$\mathrm{cm}$ MAAE rotor at $\lambda=6.1$ and $R e_{t i p}=24.100$ over the course of 6 revolutions. The naming convention established earlier is maintained here where the vortices are labeled in groups from $A$ to $F$. where the vortices from each revolution corresponds to a unique letter and the oldest vortices are labelled with $\mathrm{A}$. The numbers associated with each letter correspond to the blade number that shed a particular vortex. The edge-detected streaklines identify three large structures on the right side of the image (groups A. B, and C) that are each composed of three merged tip vortices. Vortices D1 and D2 have paired and are in the process of coalescing with vortex D3. while the pairing process is being initiated between vortices $\mathrm{E} 1$ and $\mathrm{E} 2$. 


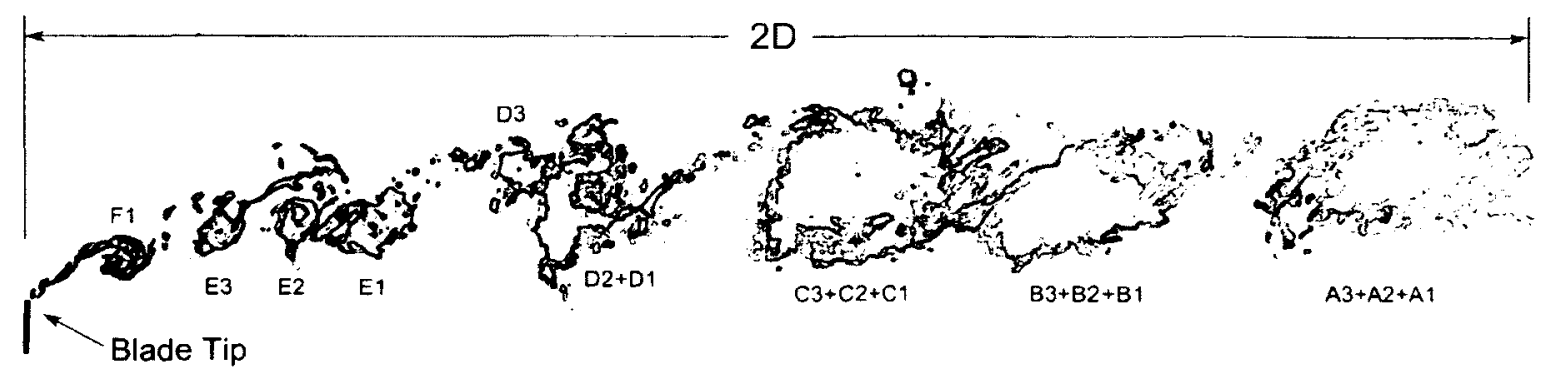

Figure 4.7: Merged tip vortices in the near wake.

The tip vortex pairing process downstream of a rotor will depend on the proximity and strength of the vortices as well as the velocity gradient that exists between the freestream and the wake. As a result. the number of blades on a rotor. the rotor solidity. the tip speed ratio, and the Reynolds number under consideration will all affect the tip vortex pairing. Tip vortex pairing was observed by Alfredsson and Dahlberg approximately 1.5D downstream of a two-bladed rotor operating with $\lambda \approx 4$ and a Reynolds number on the order of $10^{4}[27]$. This behaviour was also observed by Whale et al. approximately 1D downstream of a 2-bladed rotor operating at $\lambda \approx 6$ and a Reynolds number on the order of $10^{3}$ [49]. The onset of the pairing behaviour in the current work occurred at a downstream distance of $0.45 \mathrm{D}$. The earlier onset of pairing in the current work is attributed to the three-bladed design and the high rotor solidity compared to large-scale rotors. While it is unclear how this pairing behaviour extrapolates to full-scale conditions, recent large eddy simulations conducted by Ivanell et al. demonstrated that tip vortex pairing could occur in simulations of large-scale rotors [164]. The computation of the Tjæreborg rotor in Ref. [164] demonstrated tip vortex pairing $1.75 \mathrm{D}$ downstream of the three-bladed rotor at $\lambda \approx 7$.

The structure of the wake was further investigated using the computational results from GENUVP. The wake was visualised using the location of the discrete vortex particles shed from the trailing edge of the rotor blades and the blade tips. The wake structure when Blade 2 is passing through a $0^{\circ}$ azimuth angle can be seen in Figure 4.8 . The vortex particles inboard of the blade tip have been omitted in Figure 4.8 for clarity and, as a result. the 


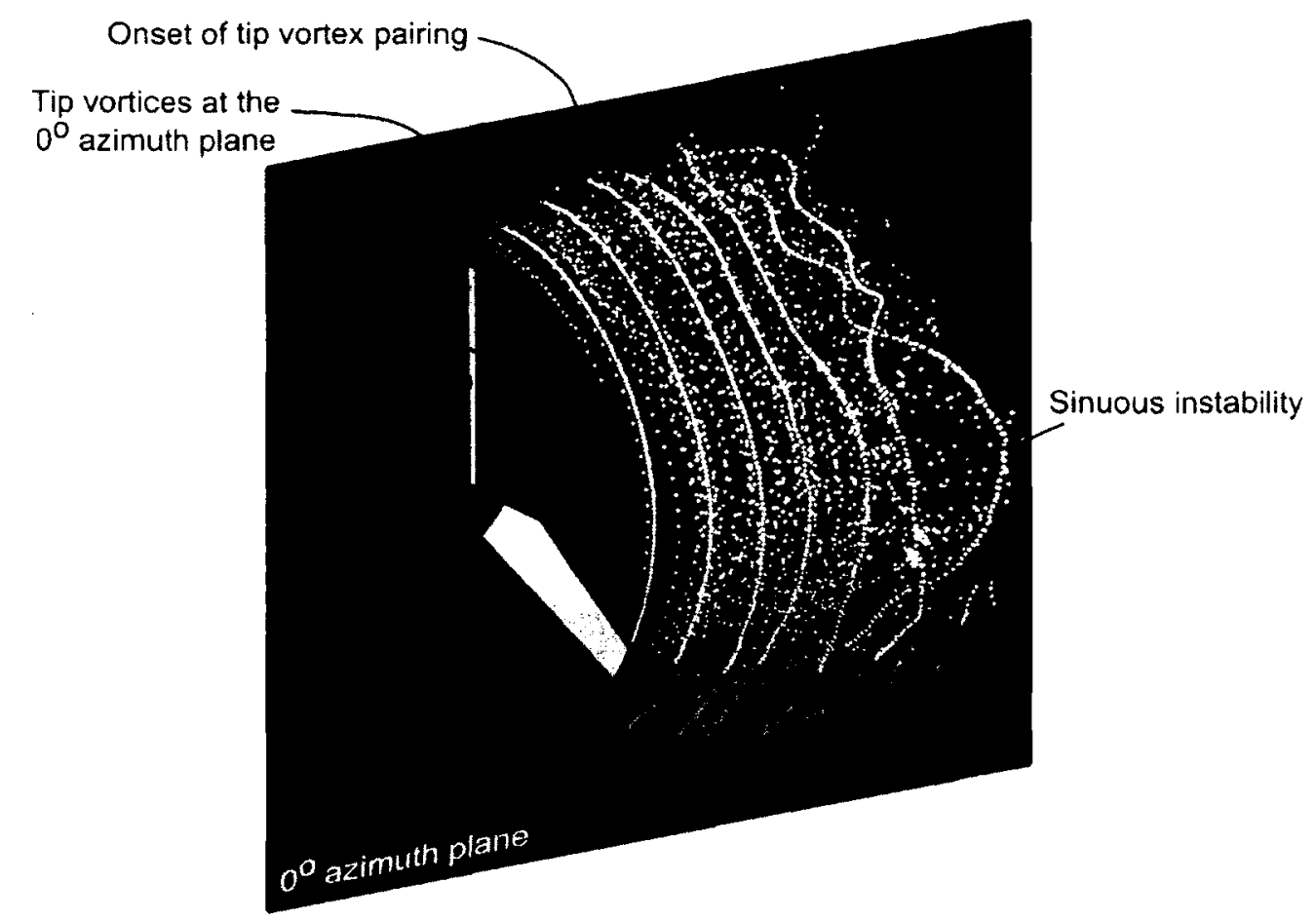

Figure 4.8: Computationally-predicted tip vortex structures - Blade 2 is at a $0^{\circ}$ azimuth angle.

visualised vortex particles highlight the helical tip vortices that roll-up downstream of the blades. The shaded plane Figure 4.8 indicates the $0^{\circ}$ azimuth plane and five tip vortex core locations are identified at this plane. The tip vortex core pattern that is observed at the $0^{\circ}$ azimuth plane in Figure 4.8 is similar to that observed in the experimental flow visualisation in Figure 4.6(b). The visualisation of the wake in GENUVP also highlights the instabilities that are present in the helical near wake region. The tip vortex pairing instability is highlighted in Figure 4.8 through the pairing of the $3^{\text {rd }}$ and $4^{t / h}$ vortices. Another common instability mode in helical vortices is a sinuous instability. This is highlighted in Figure 4.8 and results in a streamwise oscillation of the tip vortices. The magnitude of this sinuous instability is similar to the pitch distance between successive tip vortex cores. Therefore. this sinuous instability may at times reduce the separation between successive tip vortices and accelerate the tip vortex pairing process that was observed downstream of the rotor. 


\subsection{Effects of blockage on the initial wake expansion}

It was established through the literature review in Chapter 2 that blockage in wind turbine experiments with single rotors has ranged from $1 \%$ to $53 \%$ and that there is little consensus on what constitutes a tolerable amount of blockage as far as the wake development is concerned. The primary objective of this aspect of the thesis research involved identifying the effects of blockage on the initial wake expansion behind a scaled wind turbine and to establish a blockage limit that should not be exceeded if near wake properties are to be measured.

These blockage effects were investigated in the RHRC 2436 water channel using quantitative dye visualisation. The five rotors with diameters ranging from $20 \mathrm{~cm}$ to 40 $\mathrm{cm}$ shown in Figure 4.9 were used in the experiment and these rotor sizes correspond to blockage that ranges from $6.3 \%$ to $25.4 \%$. As was previously-mentioned. blockage is commonly defined in wind turbine experiments as the ratio of the rotor swept area to the cross-sectional channel area $\left(A_{x}\right)$.

A summary of the test conditions used for the rotors in the blockage test can be seen in Table 4.1 . The blockage ranged from $6.3 \%$ to $25.4 \%$ and the tip clearance is the minimum normalized distance between the blade tip and the side walls of the channel. A tip speed ratio of 6 , consistent with full-scale turbines, was targeted for all of the tests and the flow speed ranged from $0.10 \mathrm{~m} / \mathrm{s}$ to $0.23 \mathrm{~m} / \mathrm{s}$. Note that the tip speed ratio for the $20 \mathrm{~cm}$ diameter rotor test could not be maintained at $\lambda=6$, and therefore the resulting pitch distance between tip vortex cores will be increased relative to the other four test cases. The tip speed ratio had an estimated uncertainty of $\pm 3.3 \%$ from the mean value in each blockage test. The Reynolds number was maintained at approximately 23.000 in all of the blockage experiments. Another parameter that was considered in the water channel tests was the Froude number. The non-dimensional number should be maintained in the subcritical 


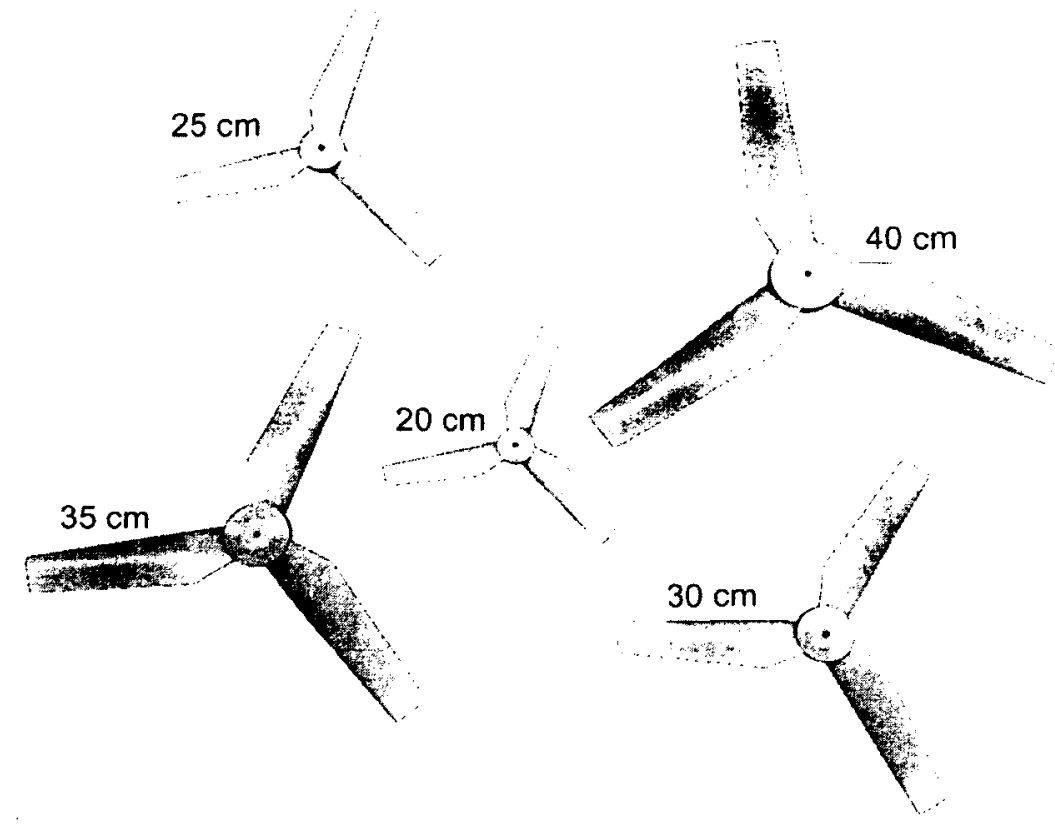

Figure 4.9: Scaled MAAE turbine blades.

flow regime in channel flow $(<1)$ if an analogy is to be maintained with subsonic flow in air. The Froude numbers based on the tested flow speeds and a channel depth of $0.81 \mathrm{~m}$ ranged from 0.036 to 0.082 and were all in the desired subcritical flow regime. Additionally. the average water temperature throughout the experimental campaign was maintained at $21.5^{\circ} \mathrm{C} \pm 0.2^{\circ} \mathrm{C}$. It has been assumed that the small variation in the temperature between the tests was not a significant source of uncertainty and, as a result. the kinematic viscosity of water at $21.5^{\circ} \mathrm{C}$ was used for Reynolds number

The effect of $R e_{t i p}$ on the wake expansion was evaluated in a separate measurement campaign that will be presented in Chapter $\bar{\jmath}$. Based on those results. it is expected that the Reynolds number variation in the current experiment. which is in the range of 23. $400 \pm 700$. will not contribute to the variation between the test cases that occurs as a result of blockage. Although the Reynolds number based on the tip conditions is used in this thesis, it should be noted that the Reynolds number can alternatively be based on the freestream speed and the turbine diameter $\left(R e_{D}\right)$. The $R e_{D}$ values associated with this 
experiment are provided in Table 4.1 for completeness.

Table 4.1: Test conditions for the blockage experiments.

\begin{tabular}{|c|c|c|c|c|c|c|}
\hline Test Number & Diameter & Blockage & Tip Clearance & $\lambda$ & $R e_{\text {tip }}$ & $R e_{D}$ \\
\hline \hline 1 & $20 \mathrm{~cm}$ & $6.3 \%$ & $1 \mathrm{D}$ & 5.8 & 23.700 & 48,500 \\
\hline 2 & $25 \mathrm{~cm}$ & $9.9 \%$ & $0.72 \mathrm{D}$ & 6.1 & 24.100 & 48,500 \\
\hline 3 & $30 \mathrm{~cm}$ & $14.3 \%$ & $0.5 \mathrm{D}$ & 6.0 & 23.700 & 48,300 \\
\hline 4 & $35 \mathrm{~cm}$ & $19.4 \%$ & $0.375 \mathrm{D}$ & 5.9 & 23.100 & 48.200 \\
\hline 5 & $40 \mathrm{~cm}$ & $25.4 \%$ & $0.26 \mathrm{D}$ & 6.0 & 22.600 & 46.200 \\
\hline
\end{tabular}

The initial wake expansion is characterised as the lateral position of the tip vortex cores and is presented in Figure 4.10 as a function of the downstream distance for the five tested rotors. The downstream and lateral distances indicated in Figure 4.10 are relative to the blade tip. The lines connecting the vortex core locations in Figure 4.10 and in all subsequent tip vortex figures are for representation only. All of the images that were used for vortex core identification correspond to instances where Blade 2 was passing through the dye stream in the $0^{\circ}$ azimuth position, similar to the case presented earlier in Figure $4.6(\mathrm{~b})$.

In addition to the wake expansion, the tip vortex pairing process is highlighted in Figure 4.10 by investigating the $3^{\text {rd }}$ and $4^{\text {th }}$ vortices for each rotor. The fourth tip vortex core in all but the $40 \mathrm{~cm}$ diameter case moves inward toward the blade tip. This is not indicative of overall wake contraction, but represents the onset of the tip vortex pairing instability that was highlighted in Figure 4.6 and highlights one of the limitations of flow visualisation for inferring the wake expansion profile. Once the vortices pair, diffuse. and grow. their presence can be observed qualitatively in the water. but the unique cores are no longer visible.

Several trends are visible in Figure 4.10 that highlight the effect of blockage on the wake expansion. The wake narrows as blockage is increased. based on the expansion observed at the fifth vortex cores. This result was expected. as the obstruction created by the rotating 


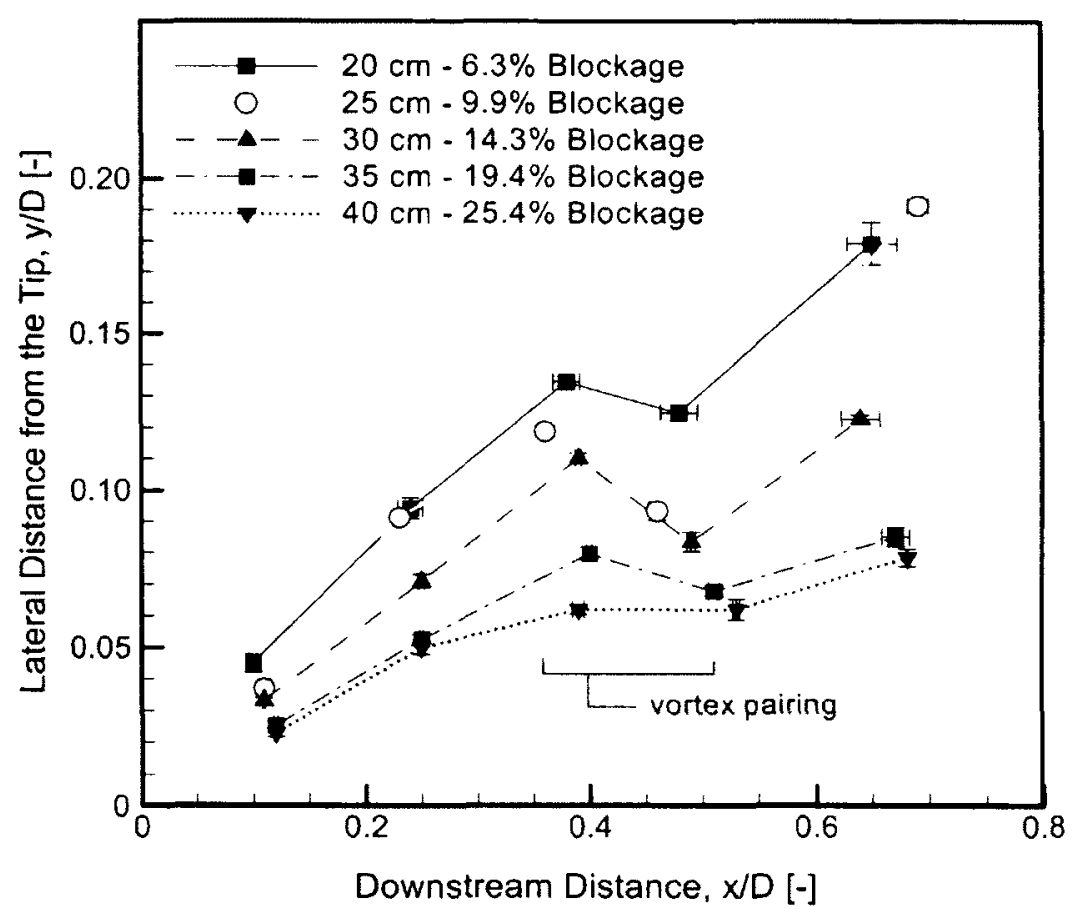

Figure 4.10: Initial wake expansion as a function of blockage at $R e_{t i p} \approx 23.000$ and $\lambda \approx 6$ $(\lambda=5.8$ for the $20 \mathrm{~cm}$ rotor $)$.

turbine in the closed test section will cause the flow between the blade tips and the solid walls, as well as the flow upstream of the rotor. to accelerate. This acceleration will cause the downstream wake to narrow artificially. Blockage had a pronounced effect on the wake expansion at the fifth vortex core when the blockage was greater than $10 \%$ and resulted in a wake which was $35 \%$ to $55 \%$ narrower than the wake downstream of the two smallest rotors. The $20 \mathrm{~cm}$ and $25 \mathrm{~cm}$ rotors have similar initial wake expansion profiles based on the first. second, and fifth vortex core locations. The vortex pairing. however, is not as prominent in the $20 \mathrm{~cm}$ diameter rotor case. This is attributed to the tip speed ratio of the test performed with the $20 \mathrm{~cm}$ diameter rotor, which could only reach $\lambda=5.8$ and resulted in a larger pitch distance between successive vortices. Despite the moderate difference observed in the behaviour of the third and fourth vortices. the initial wake width at a downstream distance of $x / D=0.65$, approximately at the fifth vortex core. does not appear to be significantly affected in this case when the blockage is less than $10 \%$. 
Increased blockage is also seen to limit the vortex pairing motion. For the smaller rotors. pairing typically occurred at $x / D=0.45$. corresponding to a wake age of $360^{\circ}$. The lateral motion associated with pairing is reduced with a diameter of $35 \mathrm{~cm}$ and is not observed for the $40 \mathrm{~cm}$ rotor within the range of axial values shown in Figure 4.10. The proximity of the vortices to the wall in the case of the $40 \mathrm{~cm}$ diameter rotor restricted tip vortex movement in the radial direction and delayed the observed vortex pairing to a vortex age of approximately $720^{\circ}$.

The vertical and horizontal error bars shown in Figure 4.10 represent \pm 1 standard error from the mean vortex core location. Error bars are shown for all of the data points. but in many cases the standard error is smaller than the symbol width. One standard error in the lateral direction is typically $2 \%$ to $5 \%$ of the mean value.

The testing environment used to characterise blockage effects on the wake development differs from most experiments conducted in wind tunnels due to the presence of one free surface and three walls. The height of the free surface of the water was not observed to vary as a result of the turbine model. There were some visible surface ripples caused by the support strut and the vertical edge where the water channel contraction joins the test section. In some tidal turbine experiments, the water level will drop downstream of the turbine and this effect is related to the thrust coefficient. the blockage. and the Froude number [73]. The drop in the free surface is associated with an increase in the bypass flow speed around the object and this may have an additional blockage-related contribution [73]. Models are available in literature to predict the drop in height experienced by the free surface. The model presented by Shives in Chapter 3 was applied to the present experiment and predicted a drop in water height of less than $1 \mathrm{~mm}$ based on the thrust coefficient. blockage. and Froude numbers identified for the $20 \mathrm{~cm}$ and $40 \mathrm{~cm}$ rotors [73]. By comparison. $1 \mathrm{~mm}$ was the accuracy of the measurements for the height of the free surface. so this was consistent with the lack of change in the free surface height observed in the experiments. 
The insensitivity of the free surface was predominantly due to the low Froude number range of 0.036 to 0.082 in the present experiment compared to a value of approximately 0.3 in many tidal turbine experiments. As the additional bypass speed effect observed in tidal turbine experiments was not a factor in the present set of tests. these results are assumed to also apply to experiments conducted in a wind tunnel.

The most conservative wall distance was selected in this experiment in order to assess the blockage effects on the wake development. recognizing the fact that the test section is not square. In fact. many test sections in literature are rectangular, allowing the wake to expand differently in the vertical and lateral directions, despite the fact that blockage is generally characterised as a simple area ratio. It is expected that the wake expansion that will occur between the rotor and the free surface will be on the same order as the expansion occurring between the rotor and the bottom wall.

\subsubsection{Sensitivity to the streakline pattern}

It was previously-highlighted in Figure 4.6 that the tip vortex streakline pattern was dependent on the blade number (1.2. or 3) passing through the dye stream. The conclusions that were drawn from the above initial wake expansion analysis with Blade 2 were verified with results obtained when Blades 1 and 3 were passing through the dye stream. Similar data reduction procedures were followed to determine the blockage dependence of the tip vortex core locations. The tip vortex core patterns corresponding to Blades 1 and 3 are shown in Figures 4.11(a) and 4.11(b), respectively. The error bars indicate \pm 1 standard error of the mean downstream and lateral core locations

The wake expansion trends observed as blockage increases in Figures 4.11(a) and 4.11 (b) are similar to the trends observed with Blade 2 in Figure 4.10 and the initial wake expansion is seen to narrow as the blockage is increased. Tip vortex pairing for Blade 3 is visible in 


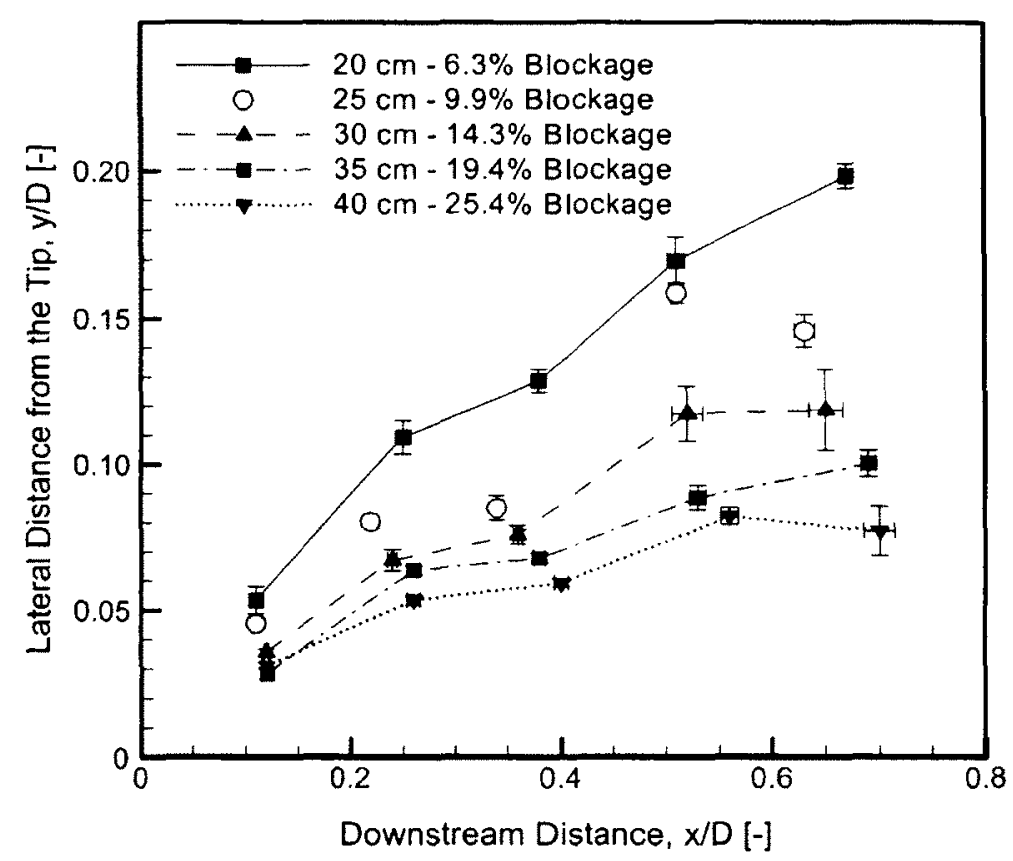

(a) Vortex core locations for Blade 1.

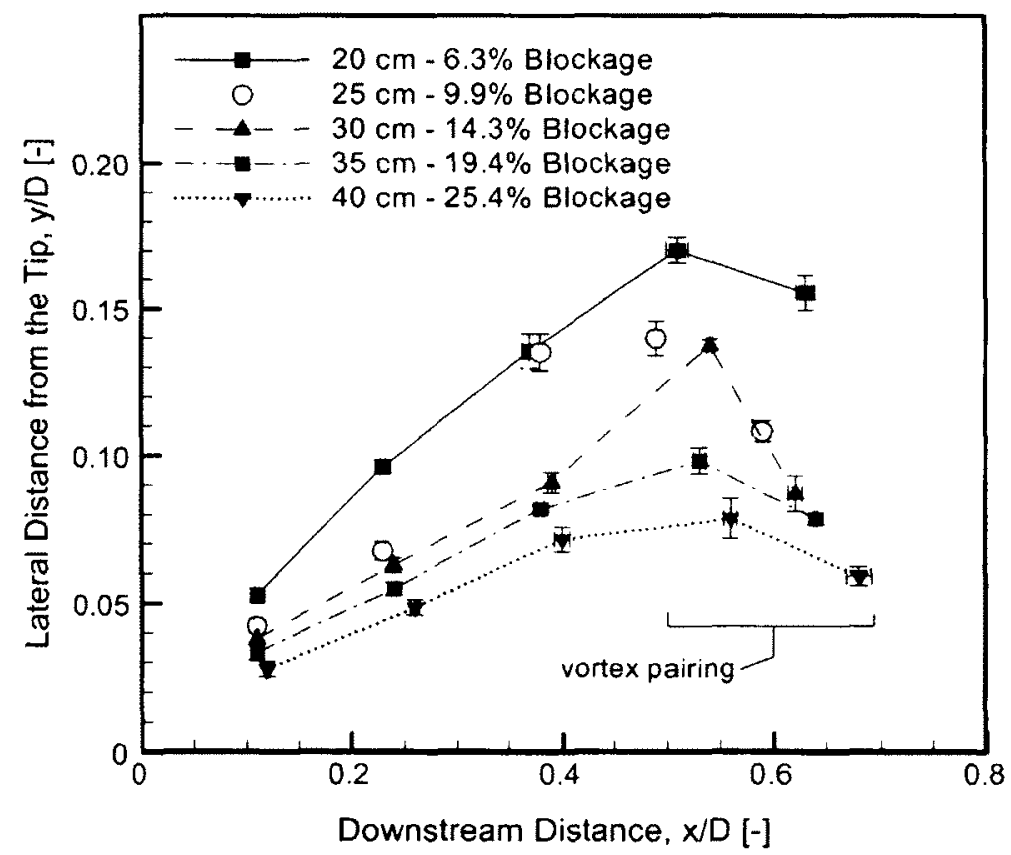

(b) Vortex core locations for Blade 3 .

Figure 4.11: Initial wake expansion variation with blade number.

Figure 4.11(b) through the motion of the $4^{t / h}$ and $5^{t / h}$ tip vortex cores. These two vortices correspond to the same two vortices that are pairing when Blade 2 was passing through the 
dye stream (though these were located at the $3^{\text {rd }}$ and $4^{t / 2}$ vortex positions in the Blade 2 streaklines). The increased vortex pairing behaviour observed with the $25 \mathrm{~cm}$ blade (where $\lambda=6.1)$ makes it difficult to directly compare the wake expansion profile to the $20 \mathrm{~cm}$ diameter rotor (where $\lambda=5.8$ ). where the vortex pairing is not as pronounced. The wake expansion, however, does appear to be somewhat affected in these two cases when blockage was $9.9 \%$. This highlights that special consideration should be given to wind tunnel blockage if wake properties are to be measured and reinforces the finding that blockage should be less than $10 \%$ to minimize wall effects on the natural expansion of the wake.

\subsubsection{Comparison with computational results}

A simulation was run in GENUVP with a model of the MAAE rotor and the resulting wake expansion was compared to the experimental blockage results in order to compare the freestream wake expansion observed in the simulations to the blockage-affected wake expansion in the experiment. For consistency with the blockage experiments, a $25 \mathrm{~cm}$ diameter rotor was simulated at $\lambda=6$ with flow conditions corresponding to $R e_{f i p}=$ 23,000. The simulation was run for 5.5 revolutions and tip vortices were extracted from the $5^{t / 2}$ revolution. A simulation consisting of 5.5 revolutions has been shown to be long enough to generate a converged solution, based on previous work [118]. Tip vortex core locations were extracted from a plane at a $0^{\circ}$ azimuth angle and the initial wake expansion profile was calculated downstream of the blade tip.

The initial wake expansion downstream of the computational rotor when Blade 2 is passing through the dye stream is compared to the experimental results with $6.3 \%$ and $9.9 \%$ blockage in Figure 4.12 because these were the closest cases to the freestream conditions applied in the simulations. The comparison highlighted in Figure 4.12 indicates that the computation predicts many important flow features. including vortex pairing between 
the $3^{\text {rd }}$ and $4^{\text {th }}$ vortices and the magnitude of the wake expansion. There is an offset along the $x / D$ axis between the computational and experimental results. however. that is consistent with the expansion behaviour previously-noted in Figure 4.10. The computation was conducted in freestream conditions and the resulting wake is representative of the wake development in the absence of walls or blockage. The difference in the tip vortex pitch distance between the experimental and computational results may be due to the fact that the simulation was conducted with a rigid blade model whereas the experimental rotor blades exhibited some deflection in the water channel. Experiments available in literature have shown that a change in the tip pitch angle can lead to an increased pitch distance between vortex cores on the order of the difference observed between the current computational and experimental results [40]. The similarities between the computation and the experiment indicate, however, that the development of the near wake in the experiment was not significantly altered when the blockage was less than $10 \%$.

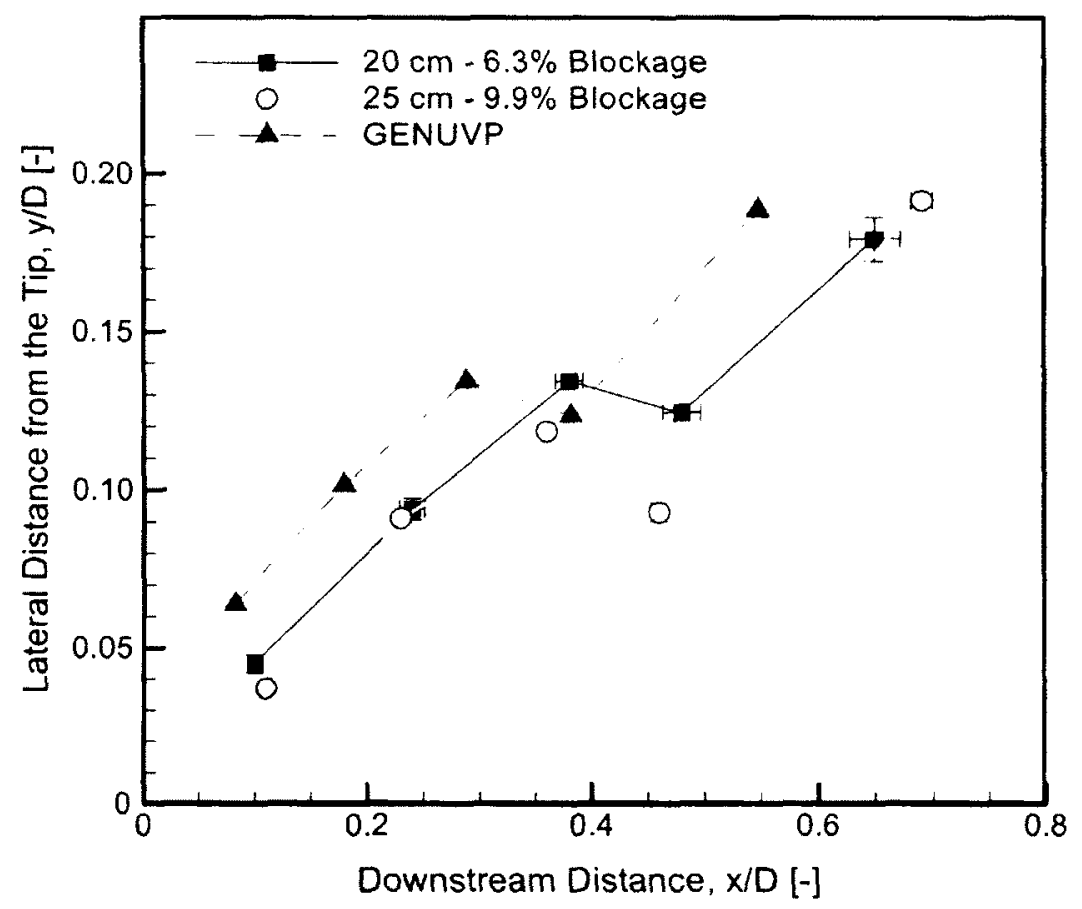

Figure 4.12: Comparison between the experimental and computational wake expansion. 


\subsection{Effect of blockage on thrust forces}

In addition to narrowing the wake. the freestream acceleration caused by blockage will cause forces and moments generated by the rotor to increase as well. Corrections have been developed in literature to account for the increase in thrust and power coefficients resulting from increased blockage and these have been shown to be effective even when blockage reaches $50 \%[30,32]$. A relationship between an increase in the thrust coefficient and a narrowing wake as a result of blockage has not been identified in literature, however. For that reason, the thrust coefficient was determined in each of the current configurations and was related to the lateral position of the tip vortices.

\subsubsection{Corrected Force Coefficients}

The thrust coefficient was calculated based on the scale measurements recorded for the five rotors and was corrected to account for the presence of the rotor and the wake in the channel, using the method recalled here in Equation (4.1), where $C_{T, U}$ and $C_{T, C}$ are the uncorrected and corrected thrust coefficients, respectively, and $\epsilon_{B}$ is the blockage factor. The blockage factor is dependent on the rotor diameter. the cross-sectional test section area and $C_{T, U}$. The mean uncorrected thrust coefficients were calculated based on $M_{x}$ obtained from the balance for each of the blockage test conditions identified in Table 4.1 and the blockage correction was used to determine $C_{T, C}$. These two coefficients are plotted with increasing blockage in Figure 4.13 .

The force measurement campaign was conducted separately from the flow visualisation study. The thrust coefficients in Figure 4.13 were calculated as the average of the mean thrust coefficients from three separate experimental runs. consistent with the procedures identified in Ref. [40]. 


$$
C_{T, C}=C_{T, U} \epsilon_{B}^{2}
$$

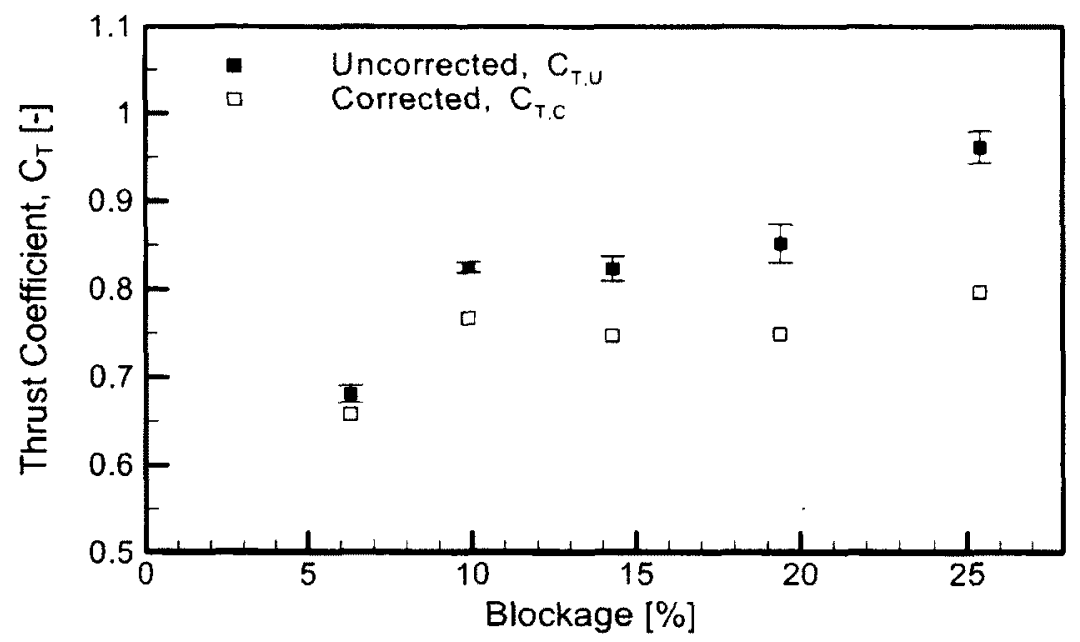

Figure 4.13: Uncorrected and corrected thrust coefficients at $R e_{t i p} \approx 23.000$ and $\lambda \approx 6$.

Figure 4.13 demonstrates that although $C_{T, U}$ increases with increasing blockage. the corrected $C_{T, C}$ values collapse to similar freestream levels. The increase in $C_{T, U}$ with increasing rotor size is associated with increased blockage effects caused by the rotor and the corresponding flow acceleration in the test section. The corrected thrust coefficient obtained with the smallest rotor (lowest blockage) does not follow the trend established by the four larger rotors. This decrease is attributed to the operating condition of the smallest rotor, which had a tip speed ratio of 5.8 . The error bars on the uncorrected coefficients in Figure 4.13 represent \pm 1 standard deviation from the mean, uncorrected. thrust coefficients, where one standard deviation from the mean represents $1 \%$ to $4 \%$ of the mean thrust coefficients.

The thrust coefficient and the lateral position of the tip vortices at a downstream distance of $x / D=0.65$ were then both evaluated with respect to the blockage in the water channel. This distance corresponds approximately to the location of last available vortex core and the lateral position at $x / D=0.65$ was interpolated based on the coordinates 
of vortices 4 and 5 for the case where Blade 2 was passing through the dye stream. The difference in the thrust coefficient between the uncorrected and corrected values $\left(\Delta C_{T}\right)$ was calculated using Equation (4.2). The effect of blockage on both the wake expansion at $x / D=0.65$ and $\Delta C_{T}$ can be observed in Figure 4.14. The reduction in $C_{T}$ obtained with the blockage correction ranged from $3.6 \%$ to $17.5 \%$ for the smallest and largest rotors. respectively. Given the magnitude of the thrust coefficient corrections that were required in the current study. blockage corrections may be beneficial even when the blockage is approximately 5-10\%. Figure 4.14 also demonstrates that the wake expansion approaches its freestream level as the blockage in the channel is reduced. The opposite is true when blockage is increased, however, and Figure 4.14 links an artificially-narrowed wake with an increasing thrust coefficient.

$$
\Delta C_{T}=\frac{\left|C_{T, C}-C_{T, U}\right|}{C_{T, U}} \times 100
$$

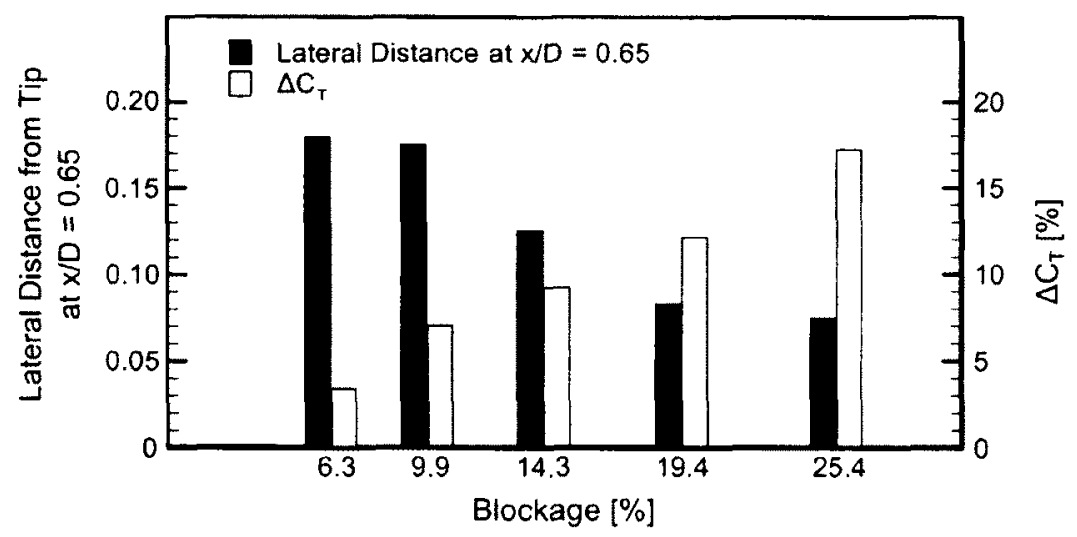

Figure 4.14: Relationship between blockage. $\Delta C_{T}$. and the wake expansion at $x / D=0.65$.

\subsection{Summary}

The current experiment was conducted in a closed-loop water channel and investigated the effects of blockage on the initial wake expansion behind scaled wind turbine rotors in 
uniform flow using quantitative dye visualisation. The results of this study set a benchmark regarding blockage effects on initial wake expansion in small-scale experiments in closed test sections. It was established that the initial wake expansion was narrowed when the solid blockage was larger than $10 \%$ for this particular three-bladed rotor at $\lambda=6$. Although this limit cannot be assumed to be definitive for all rotors. the results indicate that care must be taken in wind turbine wake experiments in order to limit the effect of blockage on the wake development. The following conclusions were developed throughout this component of the thesis:

- Guidelines were developed regarding the dye visualisation setup in the water channel. It is recommended that the dye setting should be maintained at or below the 'medium' dye level used in this study. This limits the effect of the dye stream on the wake expansion and on the recorded forces. Additionally, the turbine-supporting strut should be isolated from the turbine wake using a concentric pipe.

- Quantitative dye visualisation indicated that the initial wake expansion was narrowed by the presence of the walls when the blockage of the rotor was larger than $10 \%$. The effect of blockage on the initial wake expansion was significant and resulted in an initial wake expansion that was $35 \%$ to $55 \%$ narrower when blockage was increased above $10 \%$.

- Tip vortex pairing behaviour was dependent on the quantity of solid blockage created by the rotor and blockage of $25 \%$ was observed to delay vortex pairing.

- The experimental wake expansion results with blockage ratios of $6.3 \%$ and $9.9 \%$ were compared to computational results conducted in freestream conditions. The wake behaviour in the computation was consistent with the established wake expansion trends and predicted a similar wake expansion magnitude compared to the blockageaffected experiments. 
- The thrust coefficients inferred with a six degree-of-freedom balance demonstrated that the as-measured, uncorrected thrust coefficients increased with increasing blockage. although the corrected thrust coefficients collapsed to a similar magnitude. Based on the results of the force measurements, it is recommended that blockage corrections may be beneficial even when the blockage is $5-10 \%$. The research presented a link between a narrowed wake and an increased thrust coefficient as blockage was increased. 


\section{Chapter 5}

\section{Evaluating Reynolds number}

\section{effects in scaled wind turbine}

\section{experiments}

In addition to blockage effects, scaling in wind turbine experiments presents another challenge. Commercial wind turbines typically operate with Reynolds numbers on the order of $10^{6}$, but large single rotor experiments typically only operate with Retip on the order of $10^{5}$. Wind farm experiments present an even bigger limitation on $R e_{f i p}$, as the wind turbines must be small enough to operate within a scaled atmospheric boundary layer wind tunnel and to allow adequate room in the tunnel for realistic separation distances. This generally results in experiments with $R e_{\text {tip }}$ on the order of $10^{4}$. two orders of magnitude lower than commercial-scale turbines. Little work is available in literature that investigates the impact of such low Reynolds numbers on the wake expansion.

Therefore, the objective of the current study was to evaluate the effect of the Reynolds number on the initial wake expansion and on the measured thrust coefficients of scaled 
wind turbines. The effect of the Reynolds number was evaluated by testing two different small-scale wind turbines in a water channel. This study was designed to bridge some of the knowledge gap that exists between rotors operated at Reynolds numbers on the order of $10^{4}$ and larger experiments with Reynolds numbers on the order of $10^{5}$. Specifically. the study contrasts the wake expansion and thrust coefficients of a rotor developed for a low Reynolds number testing regime with the characteristics of a rotor that has been geometrically-scaled and operated in a low Reynolds number regime. The scaled rotor results will help to develop guidelines for scaled wind turbine testing and will explore the extent to which the scalability of the wake profile and thrust coefficients are dependent on the behaviour of an airfoil in scaled experiments.

\subsection{Scaled rotors}

The measurement campaign was conducted with the three-bladed MAAE rotor and the scaled two-bladed TU Delft rotor. A photograph of the two rotors is shown in Figure 5.1. Each rotor has a diameter of $25 \mathrm{~cm}$, which corresponds to $10 \%$ blockage in the water channel based on the rotor frontal area and the cross-section of the test section. The current rotors

were tested with Reynolds numbers that were on the order of $10^{3}$ to $10^{4}$. The two rotors differ in that the MAAE rotor was designed for a low Reynolds number regime and the scaled TU Delft rotor was geometrically-scaled.

The MAAE rotor has been designed to generate a thrust coefficient similar to that observed on full-scale wind turbines. The rotor has an Eppler E387 airfoil that behaves well in a low Reynolds number environment and a chord that was designed to generate high enough Reynolds numbers to reduce the sensitivity of the airfoil's lift and drag properties to changes in $R e_{\text {tip }}$. 
The second rotor used in the study was a $1 / 4.8$ geometrically-scaled version of a larger. $1.2 \mathrm{~m}$ diameter wind turbine from TU Delft [52.165]. The non-dimensionalised chord and twist distributions were maintained between the medium-scale rotor and the current experiment and the rotor blades have a NACA 0012 airfoil. Two scaled TU Delft rotors were manufactured using the same rapid prototyping method as the MAAE rotor and had tip pitch angles of $0^{\circ}$ and $4^{\circ}$.
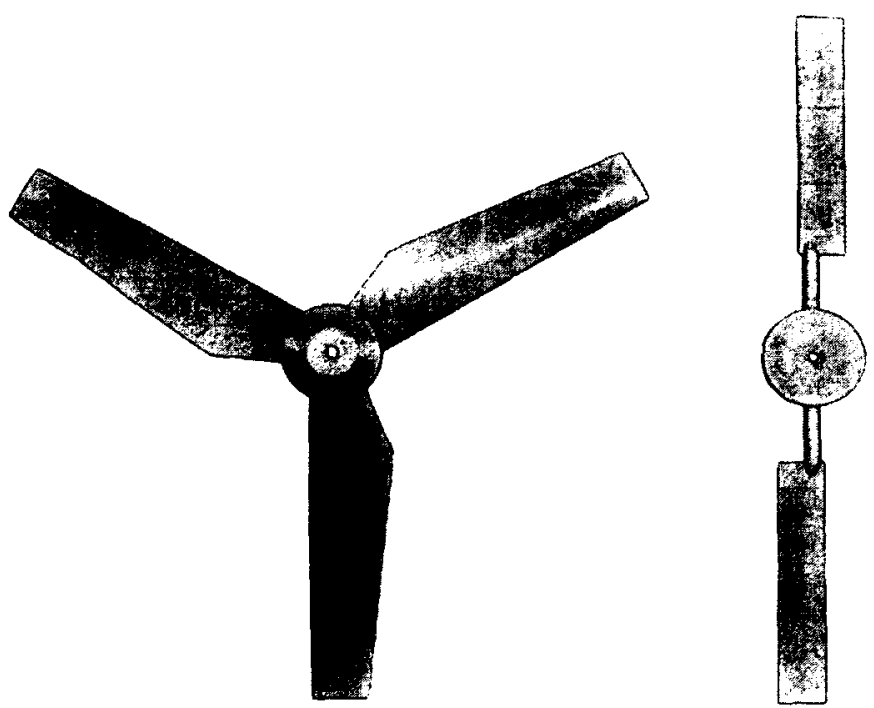

Figure 5.1: $25 \mathrm{~cm}$ diameter MAAE and TU Delft rotor blades.

\subsection{Test conditions}

The current set of experiments was conducted in the RHRC 2436 water channel at Carleton using the flow visualisation and force measurement practices established in Chapter 4. The flow speeds in the Reynolds number experiments ranged from $0.10 \mathrm{~m} / \mathrm{s}$ to $0.27 \mathrm{~m} / \mathrm{s}$ and the range Froude numbers based on these flow speeds and a channel depth of $0.81 \mathrm{~m}$ was 0.036 to 0.096 . 


\subsubsection{MAAE test conditions}

The $25 \mathrm{~cm}$ diameter MAAE rotor was operated at $\lambda \approx 6$ throughout a Reynolds number range of 3.620 to 30.100 in order to observe the effect of $R e_{t i p}$ on the initial wake expansion The lowest Reynolds number that could be tested at $\lambda=6$ was 3.620 and the highest Reynolds number that could be tested was 30.100 . The test conditions used to evaluate the Reynolds number effects are summarized in Table. 5.1.

Table 5.1: Test conditions for the MAAE rotor.

\begin{tabular}{|c|c|c|c|}
\hline Test Case & Diameter & $\lambda$ & $R e_{\text {tip }}$ \\
\hline \hline 1 & $25 \mathrm{~cm}$ & 6.0 & 3,620 \\
\hline 2 & $25 \mathrm{~cm}$ & 6.1 & 9,370 \\
\hline 3 & $25 \mathrm{~cm}$ & 5.8 & 19.100 \\
\hline 4 & $25 \mathrm{~cm}$ & 6.1 & 24.100 \\
\hline 5 & $25 \mathrm{~cm}$ & 6.1 & 30.100 \\
\hline
\end{tabular}

\subsubsection{Scaled TU Delft test conditions}

The scaled TU Delft rotor was operated in the water channel at $\lambda \approx 6$ and $\lambda \approx 8$ with Reynolds numbers ranging from 12,800 to 31.400 in order to evaluate the scalability of the initial wake expansion downstream of the rotor. The test conditions and their associated Reynolds numbers can be seen in Table 5.2. along with the medium-scale test conditions used for comparison. The tip speed ratio had an estimated uncertainty of $\pm 3.3 \%$ from the mean value in each test.

\subsection{Description of the flowfields}

Given that the two rotors have different numbers of blades and different lift-generation characteristics, their near wake flowfields will differ. Therefore. the flowfields in the near wake of the rotors will be described prior to investigating the effect of the Reynolds number 
Table 5.2: Test conditions for the scaled and medium-scale TU Delft rotor.

\begin{tabular}{|c|c|c|c|c|}
\hline Test Case & Rotor Diameter & $\theta_{\text {tip }}$ & $\lambda$ & $R e_{\text {tip }}$ \\
\hline \hline 6 & $25 \mathrm{~cm}$ & $4^{\circ}$ & 5.9 & 12,800 \\
\hline 7 & $25 \mathrm{~cm}$ & $4^{\circ}$ & 6.2 & 29,100 \\
\hline 8 & $25 \mathrm{~cm}$ & $4^{\circ}$ & 7.9 & 31.400 \\
\hline 9 & $25 \mathrm{~cm}$ & $0^{\circ}$ & 8.3 & 28.200 \\
\hline \hline & Medium-scale $[40]$ & & & \\
& $1.2 \mathrm{~m}$ & $4^{\circ}$ & 5.9 & 175.000 \\
& $1.2 \mathrm{~m}$ & $4^{\circ}$ & 7.9 & 230.000 \\
& $1.2 \mathrm{~m}$ & $0^{\circ}$ & 7.9 & 230,000 \\
\hline
\end{tabular}

on the wake expansion.

\subsubsection{MAAE flowfield}

The near wake development and the tip vortex behaviour downstream of the MAAE rotor were both described in detail in Chapter 4 . Reynolds number effects introduce some additional variations in the flowfield, however. Figure 5.2 shows images recorded at Reynolds numbers ranging from 3,620 to 30,100 . The images were recorded when Blade 2 was passing through the dye stream, and so the $3^{r d}$ and $4^{t / h}$ vortices should be in the process of pairing. based on the results presented earlier in Chapter 4 . There appears to be little vortex pairing when $R e_{t i p}=3.620$ and the pitch distance between successive vortices is larger compared to the three other cases. While the flowfield with $R e_{t i p}=9.370$ is qualitatively similar to those at the higher Reynolds numbers, the wake expansion is wider in Figures 5.2(c) and $5.2(\mathrm{~d})$.

\subsubsection{Scaled TU Delft flowfield}

Flow visualisation images were subsequently recorded with the scaled TU Delft rotors at the four test conditions highlighted in Table 5.2. In the flow visualisation experiment conducted with the MAAE rotor. significant differences were observed in the instantaneous 


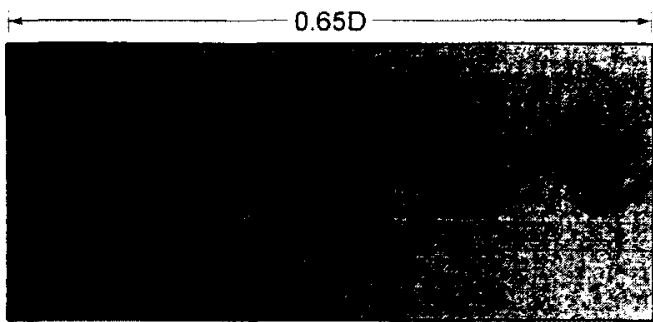

(a) Blade 2 at $0^{\circ}$ with $R e_{t i p}=3,620$.

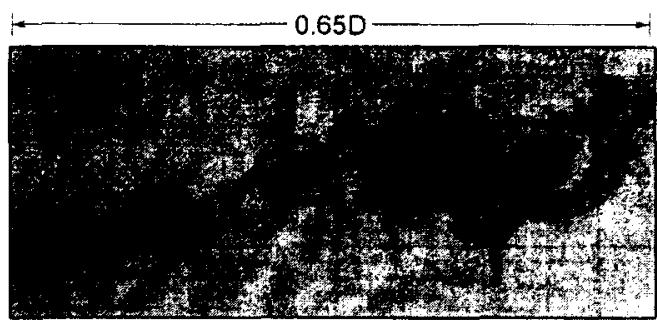

(c) Blade 2 at $0^{\circ}$ with $\operatorname{Re}_{t z p}=24,100$.

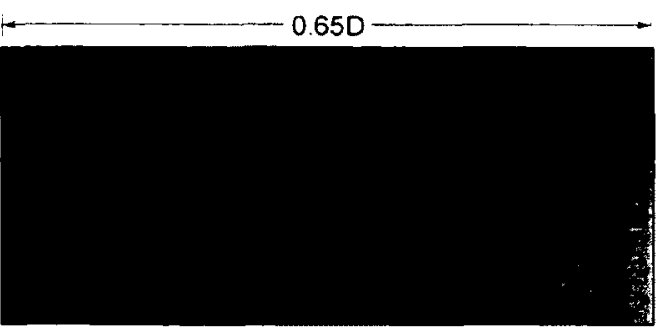

(b) Blade 2 at $0^{\circ}$ with $R_{t 2 p}=9,370$

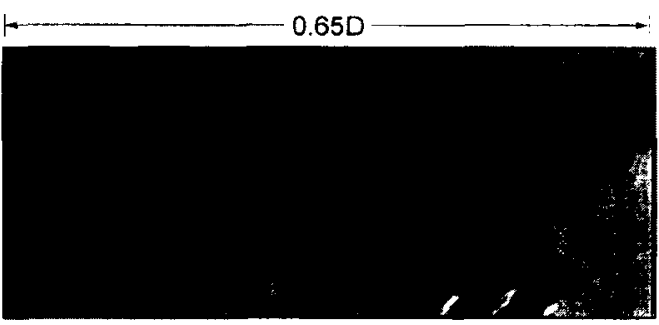

(d) Blade 2 at $0^{\circ}$ with $R e_{t i p}=30,100$.

Figure 5.2: Tip vortex visualisation downstream of the $25 \mathrm{~cm}$ MAAE rotor at $\lambda \approx 6$ at several Reynolds numbers.

flowfield when each of the three blades passed through the dye stream. However. due to the two-bladed nature of the scaled TU Delft rotor. the tip vortices are spaced further apart and the pairing behaviour observed in the MAAE test is not as prevalent. The instantaneous flowfields recorded when Blade 1 passed through the dye are shown in Figure 5.3. The tip vortex pairing instability that was present downstream of the MAAE rotor is not visible in these images, however. and occurred further downstream.

The flowfield downstream of the rotor was. however. dependent on the rotor operating conditions. The images in Figure 5.3 have the same horizontal and vertical scale and portions of the downstream flowfield were omitted in Figures 5.3(a) and 5.3(b) in order to focus the field of view on the first five tip vortex structures. The flow visualisation images highlight the effect of the tip pitch angle setting and the tip speed ratio on the wake expansion and the tip vortex pitch. The effect of the tip speed ratio on the tip vortices can be visualised in Figure 5.3(a). The tip vortex pitch increases from Figure 5.3(a)-i to Figure 5.3(a)-ii due to the reduced tip speed ratio in Figure 5.3(a)-ii. 
The effect of decreasing the tip pitch angle is highlighted by comparing Figures 5.3(b)-i and 5.3(b)-ii. Note that Figures 5.3(a)-i and 5.3(b)-i are the same image. The reduced tip pitch angle in the test visualised in Figure 5.3(b)-ii creates a higher effective angle of attack distribution along the blade. higher circulation, and higher shed vorticity. resulting in stronger tip vortices. The increased wake expansion that accompanies a higher lift force along the blade and higher thrust coefficient can be observed in Figure 5.3(b)-ii as well.
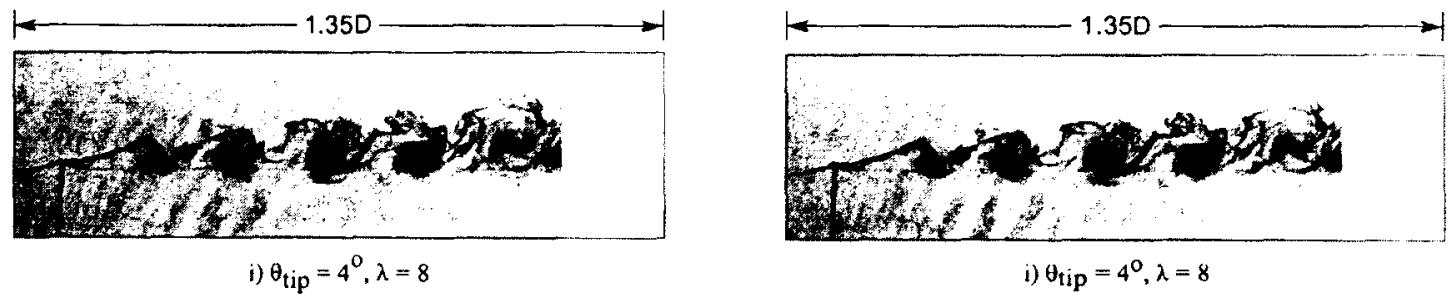

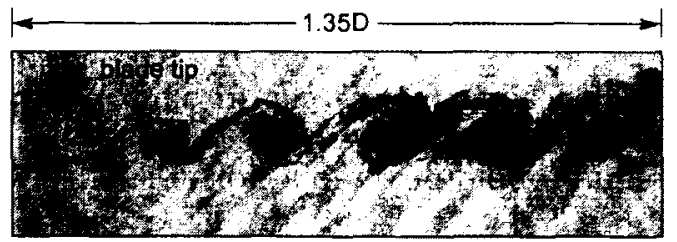

ii) $\theta_{\text {tip }}=4^{\circ}, \lambda=6$

a) Tip speed ratio effect

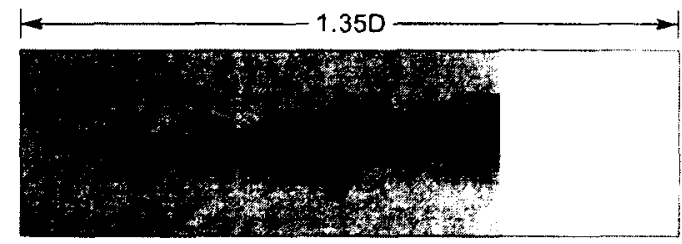

ii) $\theta_{\text {tip }}=0^{\circ}, \lambda=8$

b) Tip pitch angle effect

Figure 5.3: Scaled TU Delft tip vortex visualisation. $R e_{\text {tip }} \approx 30.000$.

The tip vortex pairing process was investigated further downstream by post-processing an image using an edge-detection filter. It was demonstrated in Chapter 4 that this technique was effective in highlighting flow structures when the dye had partially diffused downstream of the rotor. The flowfield that extends $1.85 \mathrm{D}$ downstream of the blade tip with $\theta_{t i p}=4^{\circ}$ at $\lambda=7.9$ and $R e_{t i p}=31.400$ can be seen in Figure 5.4. Six pairs of vortices are identified downstream of the blade tip and each vortex pair has been assigned a common letter following the naming conventions also applied in Chapter 4 . The onset of vortex pairing in this configuration occurs between vortices $\mathrm{C} 1$ and $\mathrm{C} 2$. approximately 1D-1.25D downstream of the blade tip. This behaviour is consistent with the tip rortex pairing distance noted by Alfredsson and Dahlberg (1.jD downstream) and by Whale et al. 
(1D downstream) using other two-bladed wind turbines [27.49].

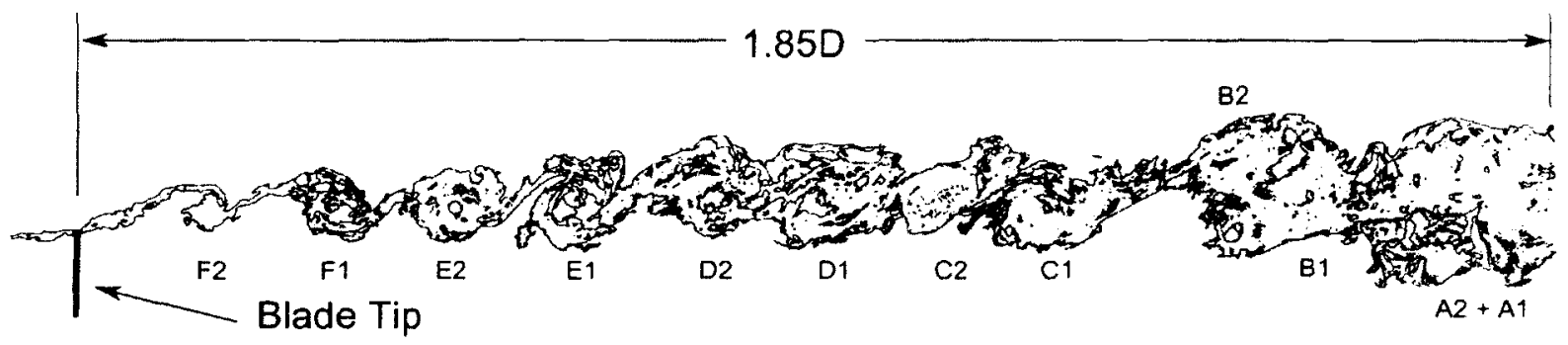

Figure 5.4: Merged tip vortices in the scaled TU Delft near wake. $\lambda=7.9 . \theta_{\text {tip }}=4^{\circ}$. $R e_{\text {tip }}=31.400$.

\subsection{Reynolds number effects on the wake expansion}

Quantitative flow visualisation was conducted downstream of the MAAE and scaled TU Delft rotors in order to gain an improved understanding of scaling effects. The initial wake expansion downstream of the MAAE rotor was assessed in order to evaluate the sensitivity of the wake to changes in the Reynolds number. This is contrasted with the wake expansion identified downstream of the geometrically-scaled TU Delft rotor when tested in a low Reynolds number regime.

\subsubsection{MAAE wake expansion}

The $25 \mathrm{~cm}$ diameter MAAE rotor was tested at the five Reynolds numbers previouslyshown in Table 5.1 in order to assess the effect of the Reynolds number on the initial wake expansion. The vortex cores that were extracted from six dye visualisation images were averaged at each Reynolds number. The initial wake expansion at each Reynolds number can be seen in Figure 5.5. The vortex core locations correspond to instances where Blade 2 passed through the dye and the blade tip position corresponds to the origin. The vertical 
and horizontal error bars shown in Figure 5.5 represent \pm 1 standard error from the mean vortex core location. One standard error in the lateral direction is typically $2 \%$ to $5 \%$ of the mean value and the horizontal error bars are generally smaller than the symbol. The errors in the lateral direction were the highest in the smallest Reynolds number case and may have been due to radial vortex wandering that was observed in this particular case. Figure 5.5 demonstrates that the wake becomes wider with increasing Reynolds number.

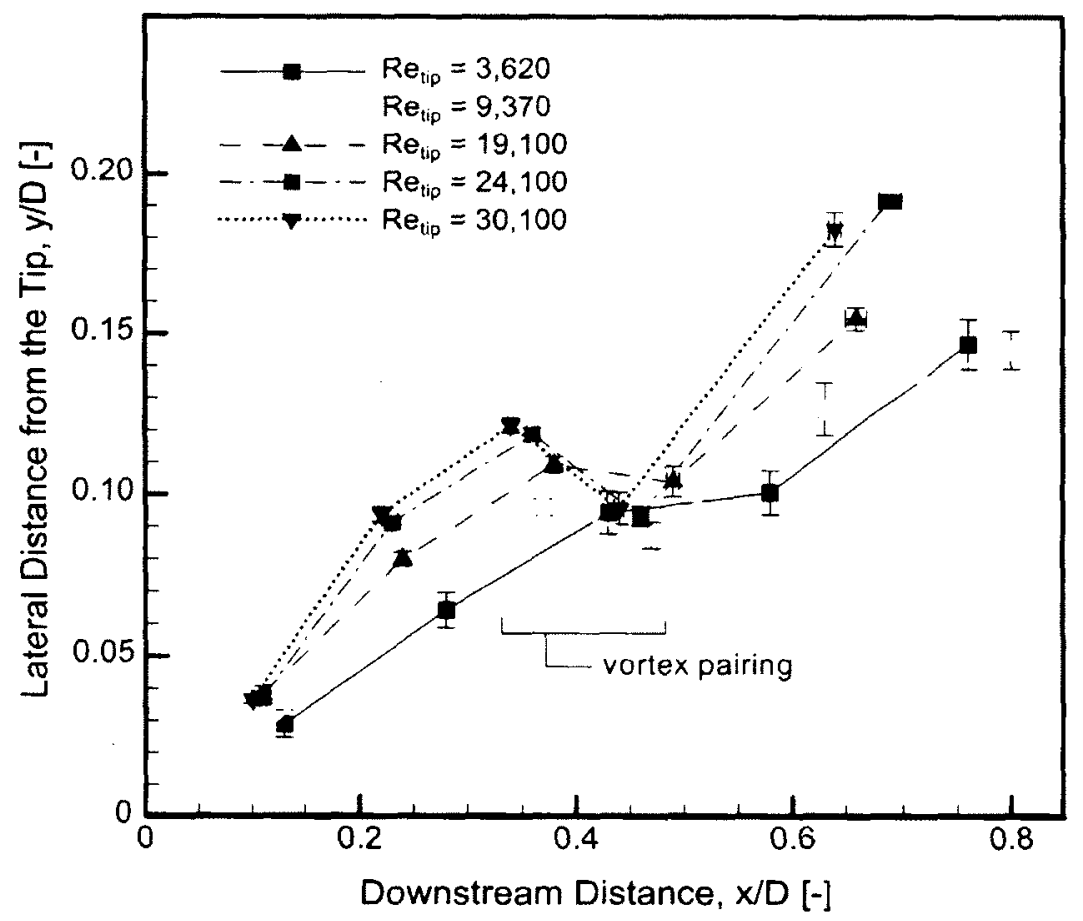

Figure 5.5: Wake expansion dependence on $R e_{t i p}$ for the MAAE rotor at $\lambda \approx 6$.

The initial wake expansion and the vortex pairing behaviour appear to become less dependent on Reynolds number when the Reynolds number was higher than 24.100. The similarity in the initial wake expansion above a Reynolds number of 24,100 may be related to the lift and drag characteristics of the E387 airfoil at these particular Reynolds numbers. Experiments presented in Refs. [150] and [151] evaluated the lift and drag coefficients of the E387 airfoil at Reynolds numbers ranging from 10.000 to 60.000 . It was found that laminar to turbulent transition occurred over smooth E387 airfoils at a Reynolds number 
of 30.000 . but that this transitional behaviour could occur at a Reynolds number of 20.000 because of surface roughness effects [151]. While the blades used in the current study are smooth to the touch. there is a visible roughness pattern on the surface of the blades that was an artifact of the rapid prototyping manufacturing method and may have affected the transitional Reynolds number of the rotor blades. The initial wake expansion could not. however, be tested above a Reynolds number of 30.100 with this particular rotor. When the flow speed was increased in an attempt to raise the Reynolds number, surface waves were generated due to the vertical edge in the water channel wall between the contraction flange and the test section glass. These surface waves blurred the view of the measurement grid located above the free surface of the channel.

\subsubsection{Scaled TU Delft wake expansion}

In a similar manner to the flow visualisation experiments conducted with the MAAE rotor, vortex cores were digitally extracted from six images at each of the scaled $\mathrm{TU}$ Delft test conditions in Table 5.2. The experimental vortex cores obtained from the dye visualisations were compared to smoke visualisation results presented by Haans et al. [40] in addition to HWA measurements behind the same rotor discussed by Vermeer [ 52$]$.

There were some small differences between the present experiment and the previous work by Haans et al. and Vermeer that should be mentioned before interpreting the results [40.52]. The first observation relates to the physical location where the data were recorded. The smoke visualisation conducted by Haans et al. measured the tip vortex core locations when the smoke and the blade were at $90^{\circ}$ and $270^{\circ}$ azimuth angles. whereas the results obtained in the present study were recorded when Blade 1 passed through the dye at a $0^{\circ}$ azimuth angle [40]. Additionally, the HWA results from Ref. [40] trace the radial location of the tip vortices at a plane and do not correspond to the tip vortex core locations based on 
streakline patterns. The second difference between the current experiment and its mediumscale equivalent relates to wake asymmetry and the observation that the wake expansion may be somewhat dependent on the azimuth angle. Wake asymmetry has been observed by Haans et al. and by Barber et al. downstream of two- and three-bladed turbines. respectively $[31,40]$. In the work by Haans et al. wake asymmetry was observed as a $5 \%$ to $10 \%$ difference in the radial location of the first two tip vortices at two different azimuth angles [40]. This difference, however, is small compared to the difference observed in the wake width downstream of the rotor in the present work as a result of a lower $R e_{t i p}$. Lastly. the original data from Haans et al. and Vermeer were normalised with respect to the rotor radius, but are normalised in the current work with respect to diameter for consistency with the blockage results from the previous chapter $[40,52]$.

The vortex cores in the near wake at Reynolds numbers of 12,800 and 29.100 at $\lambda \approx 6$ with $\theta_{\text {tip }}=4^{\circ}$ are compared to the medium-scale vortex core locations determined from HWA measurements conducted by Vermeer in Figure 5.6 [52]. Smoke visualisation results were not available for this particular case. The results in Figure 5.6 follow similar trends to those established with the MAAE rotor, in that the wake expansion widens with increasing Reynolds number. The lateral positions of the tip vortices at Reynolds numbers of 12.800 and 29,100 are approximately $60 \%$ and $30 \%$ narrower. respectively, than the positions observed in the medium-scale results [52].

The rotor with a tip pitch angle of $4^{\circ}$ was also tested with $\lambda=7.9$ and $R e_{t i p}=31.400$. The dye visualisation results from this test are compared to smoke visualisation results from Haans et al. and HWA data from Vermeer in Figure 5.7 [40.52]. A $40 \%$ to $50 \%$ difference in the wake expansion can be observed at the $3^{\text {rd }}$ and $4^{\text {th }}$ vortices by comparing the two medium-scale data sets to the current results. The differences in the HWA data and the smoke visualisation results were attributed by Haans et al. to a change in the hub shape between the two tests [40]. Additionally, Haans et al. described that the open jet wind 


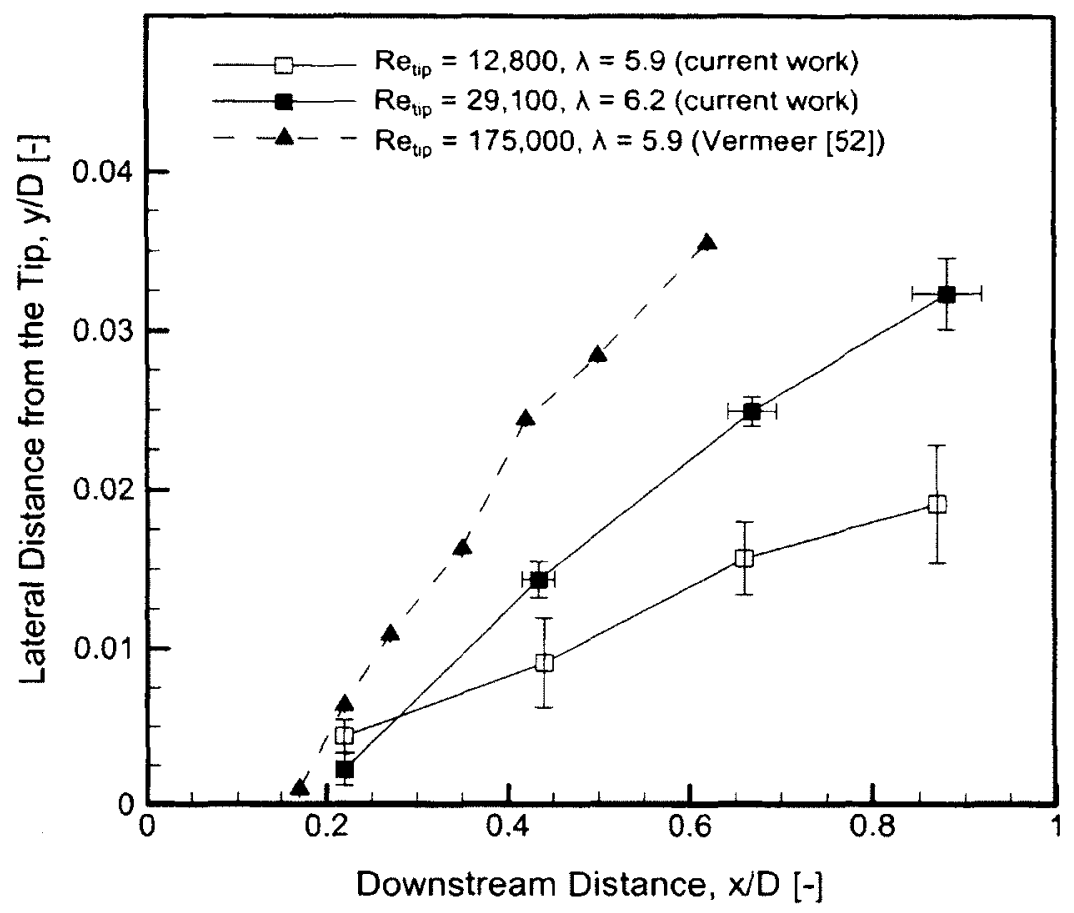

Figure 5.6: Initial wake expansion dependence on $R e_{t i p}$ for the TU Delft rotor at $\lambda \approx 6$.

tunnel was moved into a larger room for the smoke visualisation tests compared to the earlier HWA experiment by Vermeer [40.52].

Following the tests with a $4^{\circ}$ tip pitch angle. a second rotor with a tip pitch angle of $0^{\circ}$ was tested at $\lambda=8.3$ and $R e_{t i p}=28,200$. The calculated wake expansion is compared to the medium-scale experimental results in Figure 5.8 [40.52]. The results in Figure 5.8 are consistent with the results presented in the previous two figures and the wake expansion was again $40 \%$ to $50 \%$ narrower in the current experiment than in the medium-scale tests.

The set of results obtained at these test conditions was self-consistent in that the wake expansion was larger with a tip speed ratio of 8 and when the rotor had a tip pitch angle of $0^{\circ}$. The lower blade tip pitch setting will result in higher angles of attack throughout the blade span and higher circulation. a larger thrust coefficient. and a wider wake downstream of the rotor. 


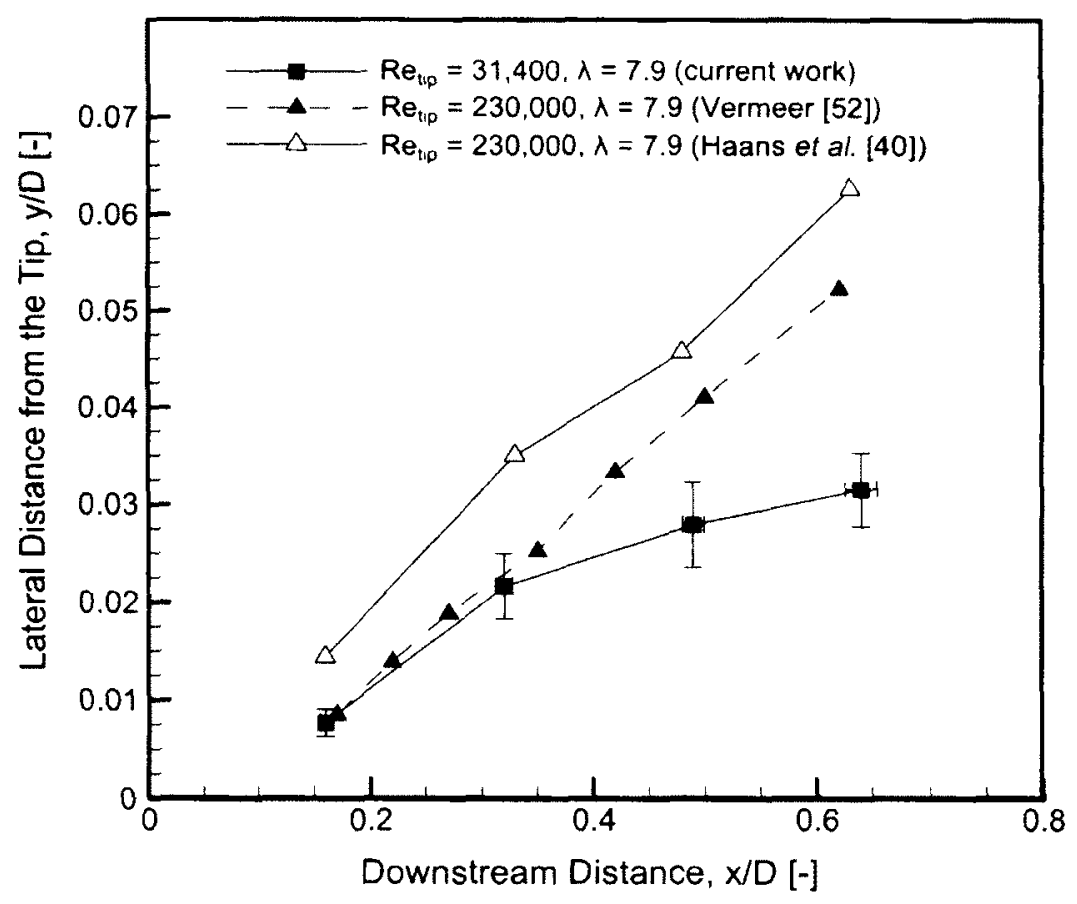

Figure 5.7: Initial wake expansion for the TU Delft rotor at $\lambda \approx 8$ and $\theta_{\text {tip }}=4^{\circ}$.

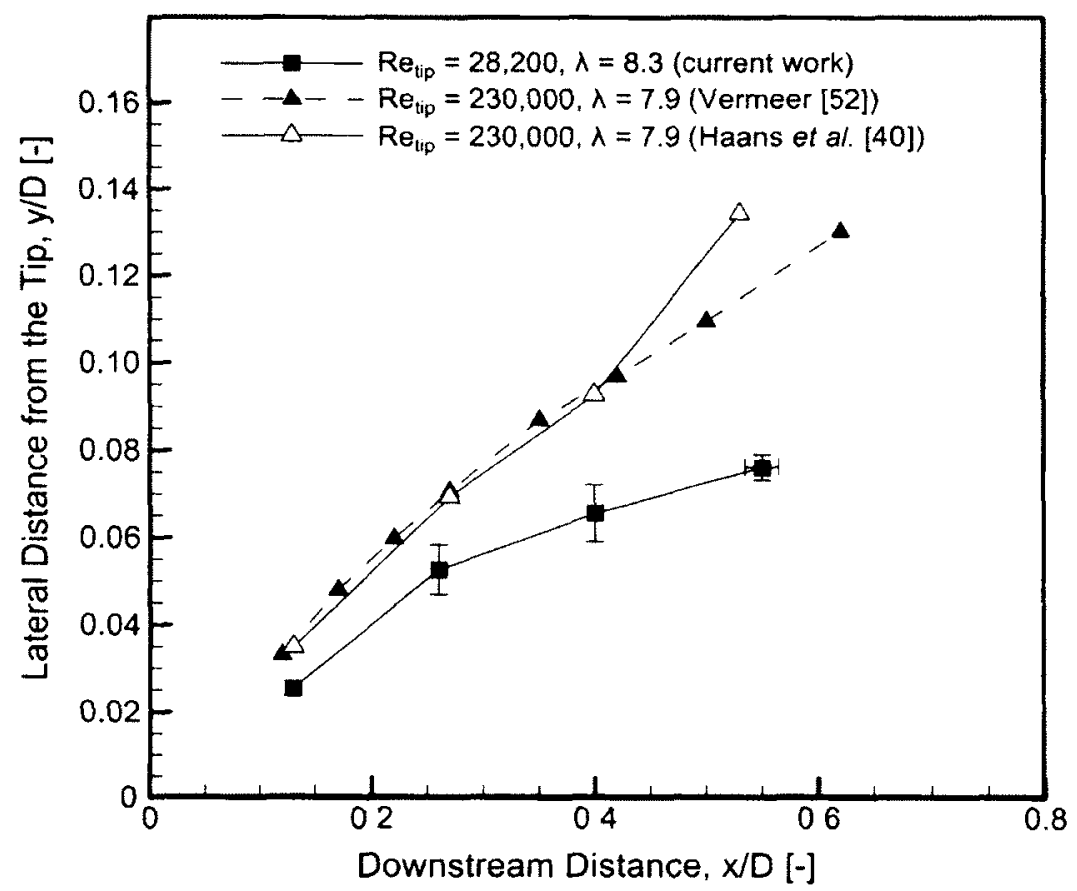

Figure 5.8: Initial wake expansion dependence on $R e_{t i p}$ for the TU Delft rotor at $\lambda \approx 8$ and $\theta_{\text {tip }}=0^{\circ}$. 
The error bars presented in Figures 5.6. 5.7. and 5.8 represent \pm 1 standard error from the mean tip vortex location. The errors observed when calculating the tip vortex core locations for the scaled TU Delft rotor were higher than those observed during the MAAE test. The errors in the downstream direction are smaller than the symbol size in many cases and represent less than $\pm 2 \%$ of the mean value. The errors in the lateral direction. however, are in most cases $\pm 5 \%$ to $\pm 10 \%$. This is partially attributed to the fact that all of the dye visualisation tests conducted on the scaled TU Delft rotor were conducted at Reynolds numbers below 31,400, where the performance of the NACA 0012 airfoil is degraded [148]. The wake expansion errors increased at lower Reynolds numbers in the MAAE test as well, when the E387 airfoil was operating in a Reynolds number range that resulted in diminished performance. The error bars from the medium-scale flow visualisation experiment conducted by Haans et al. were approximately $\pm 3 \%$ to $\pm 10 \%$ of the mean value and represented uncertainty in the measurements based on $95 \%$ confidence intervals [40].

The large variation between the wake expansion observed with the scaled model and the medium-scale turbine indicates one of the limitations of geometrically-scaling an existing rotor design without further tailoring it to the desired Reynolds number regime. The lift, drag, and separation properties of the NACA 0012 airfoil are significantly different at Reynolds numbers on the order of $10^{4}$ than at Reynolds numbers on the order of $10^{5}$. Experiments conducted on a NACA 0012 airfoil at a Reynolds number of 13.000 by Hung and Ngoc measured the laminar separation location as the angle of attack was increased [166]. Laminar separation occurred over the airfoil and there was no evidence of laminar-to-turbulent transition [166]. Direct Numerical Simulations of a NACA 0012 airfoil at a Reynolds number of 50,000 performed by Lehmkuhl et al. indicated that a long laminar separation bubble was present over approximately $50 \%$ of the chord of the airfoil and that the resulting turbulent flow reattached [167]. The formation of a small laminar separation bubble and the associated laminar-to-turbulent transition over the upper surface of an airfoil 
has been observed at Reynolds numbers on the order of $10^{5}$ by Tanaka [168]. Additionally. the experimental work of Laitone has indicated that the maximum lift coefficient $\left(C_{L \max }\right)$ of a NACA 0012 airfoil at a Reynolds number of 20.700 was approximately 0.45 and occurred at a low angle of attack of $5.5^{\circ}[148]$. By comparison, the NACA 0012 airfoil has been demonstrated to have a $C_{L \max }$ of 0.85 at an angle of attack of $9^{\circ}$ at Reynolds number of 160,000 [169]. These previous results available in literature indicate that purely laminar separation may in fact be occurring over the scaled wind turbine blades at low angles of attack. The extent of the flow separation and turbulent flow over the airfoil will affect the lift production of the blades. the tip vortex formation, and the overall wake expansion. Additionally, the reduced lift and associated increased drag at low Reynolds numbers will further alter the performance of the scaled model. The results of the current flow visualisation study demonstrate the importance of reaching a Reynolds number where airfoil properties, such as the lift curve slope, $C_{\text {Lmax }}$. and the separation characteristics become less dependent on changes to the Reynolds number.

Following the investigation of the wake expansion. the effect of the Reynolds number on the tip vortex pitch was evaluated. The tip vortex pitch was approximated as the downstream spacing between the blade tip and the $2^{\text {nd }}$ tip vortex core. which corresponds to a tip vortex age of $360^{\circ}$. The tip vortex pitch results obtained from the scaled TU Delft rotor tests are compared to the medium-scale tip vortex pitch distances from Haans et al. in Figure 5.9 [40]. It should be noted that the lines in Figure 5.9 are for visualisation purposes only and the shaded symbols represent the data from Ref. [40]. Figure 5.9 demonstrates that the tip vortex pitch is not particularly sensitive to the Reynolds number. At $\lambda \approx 6$ and a tip pitch angle of $4^{\circ}$, the tip vortex pitch distance obtained in the present experiment varied by a maximum of of $5.4 \%$ compared to the results from Ref. [40] at a Reynolds number of 175,000. Similarly. the tip vortex pitch obtained at $\lambda \approx 8$ and tip pitch angles of $4^{\circ}$ and $0^{\circ}$ in the current experiment varied by a maximum of $2.4 \%$ compared to the medium-scale 
results at a Reynolds number of 230.000 . These variations are on the order of the standard error of the axial vortex core location in this experiment. The tip vortex pitch. however, is predominantly a function of the blade geometry $\left(\theta_{t i p}\right)$ and the operational characteristics $(\lambda)$. The insensitivity of the tip vortex pitch to changes in Reynolds number was consistent with the MAAE results. where the tip vortex pitch was not significantly affected by changes in $R e_{t i p}$ above a Reynolds number above 9,300 .

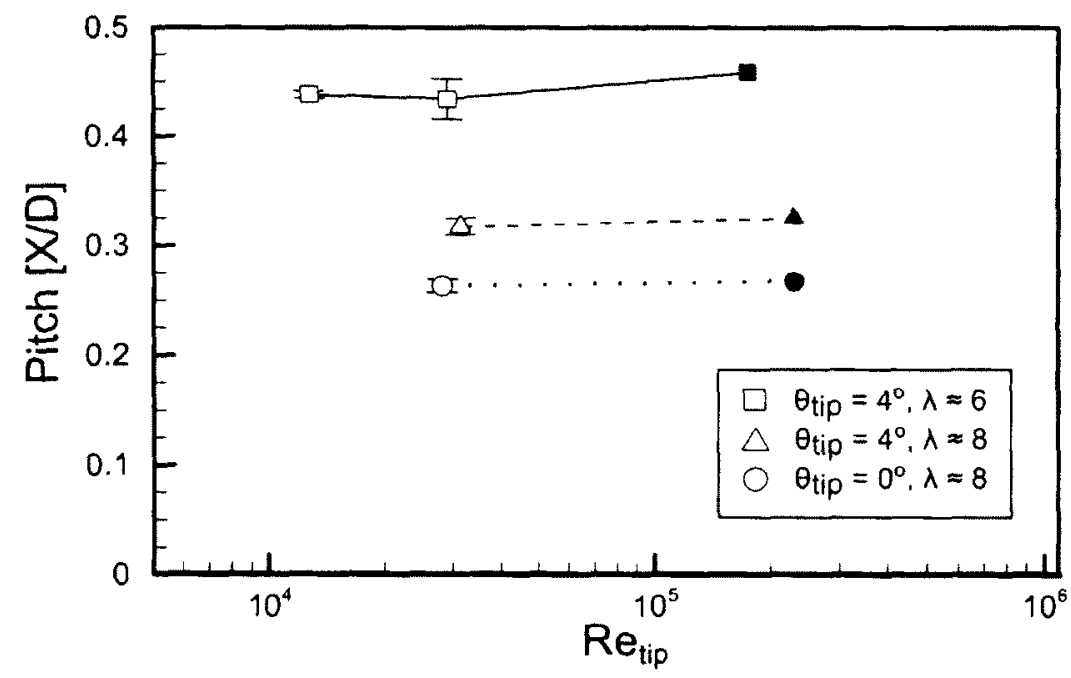

Figure 5.9: Comparison of the small-scale tip vortex pitch with medium-scale results. Filled symbols represent experimental data from Haans et al. [40].

\subsection{Effect of the Reynolds number on the thrust coefficient}

One of the operating characteristics that should be matched between full-scale and scaled wind turbines is the thrust coefficient $\left(C_{T}\right)$. The thrust coefficient is correlated to the wake deficit downstream of the turbine and is an important parameter in establishing the downstream wake recovery. Typical full-scale wind turbines have a thrust coefficient that ranges from 0.75 to 1 when $\lambda$ ranges from 6 to 8 . This section will compare $C_{T}$ generated by the low Reynolds number MAAE rotor with that produced by the geometrically-scaled turbine. 


\subsubsection{MAAE thrust coefficients - water channel}

As in the blockage measurement campaign. the thrust coefficient was calculated based on the recorded $M_{x}$ from the six degree-of-freedom balance. The thrust data were recorded at $500 \mathrm{~Hz}$ and were averaged over 30 revolutions. The mean thrust coefficient was then determined based on the thrust force averaged over three separate runs.

The thrust coefficient was evaluated at each of the Reynolds number tests conducted with the MAAE rotor and these coefficients were subsequently corrected for blockage effects. The blockage factor $\left(\epsilon_{B}\right)$ in the MAAE experiments ranged from 0.98 to 0.95 , resulting in a maximum thrust coefficient reduction of $9.1 \%$ at a Reynolds number of 30.100 . The effect of the Reynolds number on the corrected thrust coefficients and the lateral position of the tip vortices at a downstream distance of $x / D=0.65$ can be seen in Figure 5.10 . The vortex position at $x / D=0.65$ was interpolated based on the surrounding data points. As one can see, the thrust coefficient and the wake expansion both generally increase with increasing Reynolds number in Figure 5.10. The investigation of the visible trend in the wake expansion was limited, however, by the upper Reynolds number limit in the experiment of 30,100. The data point at $R e_{t i p}=3.620$ had a higher-than-expected thrust coefficient and this was attributed to the axial component of the high drag force at such a low Reynolds number. The MAAE thrust coefficient approached 0.8 at a Reynolds number of 30.100 and was in the desired range of full-scale values.

\subsubsection{MAAE thrust coefficients - wind tunnel}

The maximum speed of the water channel limited the Reynolds number range over which the thrust coefficient could be evaluated. As the wind tunnel results that will be described in Chapter 6 used a higher $R e_{\text {tip }}$. it was desired to determine $C_{T}$ over a greater range of $R e_{t i p}$. The $25 \mathrm{~cm}$ rotor was therefore installed in the upstream test section of the ABL 


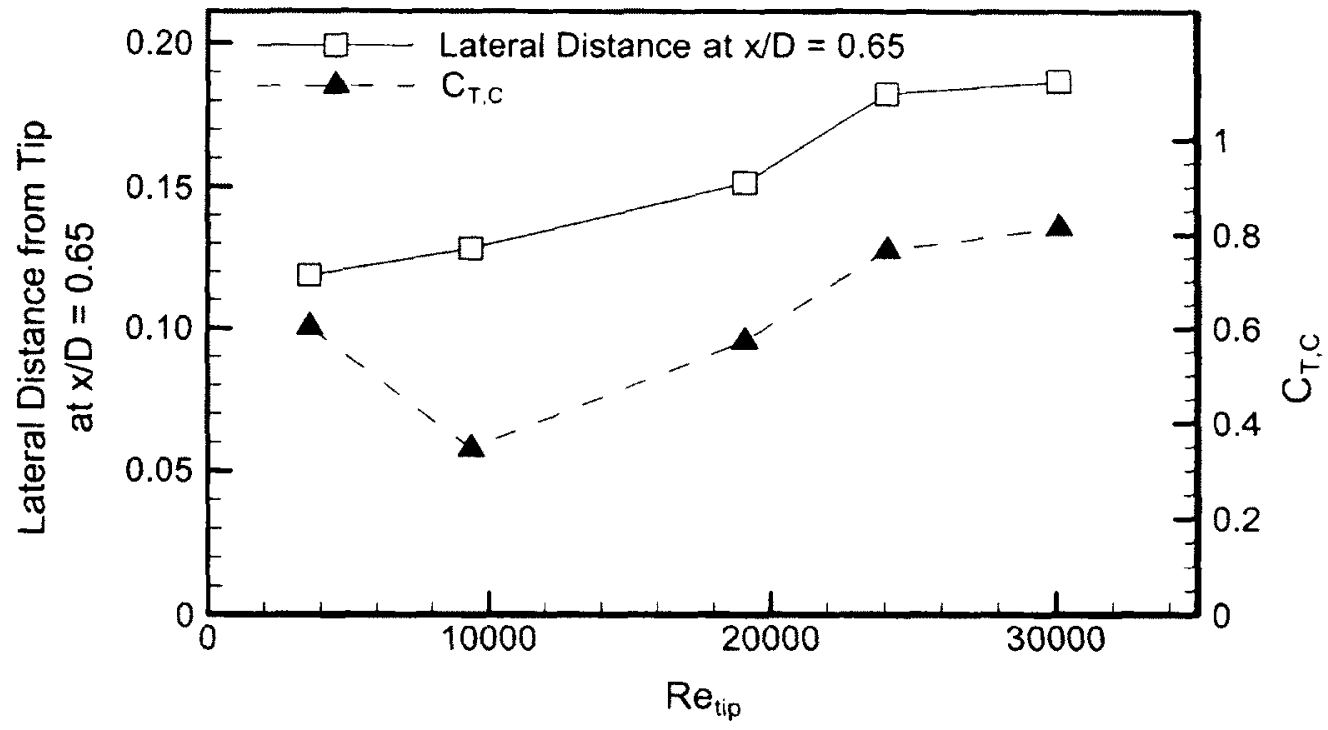

Figure 5.10: Relationship between the Reynolds number, $C_{T}$, and the wake expansion for the MAAE rotor.

wind tunnel and $C_{T, C}$ was calculated using data from the six degree-of-freedom balance at a higher Reynolds number range. A schematic of the wind turbine installed in the upstream test section of the wind tunnel is shown in Figure 5.11. Each data point obtained in the wind tunnel represents the average of two separate runs and the subsequent mean thrust coefficient was corrected for blockage effects for consistency with the water channel results. despite the observation that the blockage corrections represented $2 \%$ to $3 \%$ of the mean $C_{T}$.

The $C_{T, C}$ determined in the wind tunnel is compared to the values that were previouslypresented from the water channel experiments in Figure 5.12. Reynolds number similarity could be achieved between the two facilities and so the results at $R e_{t i p} \approx 30.000$ could be compared and demonstrate good agreement. The thrust coefficient at a Reynolds number of 38,500 was 0.92 , and the wind turbines used in the experiment described in Chapter 6 were operated near this condition. The tip speed ratio could not be maintained at 6 when the Reynolds number was 47.000 and this will increase $C_{T, C}$. but the uppermost data point was included for completeness. 


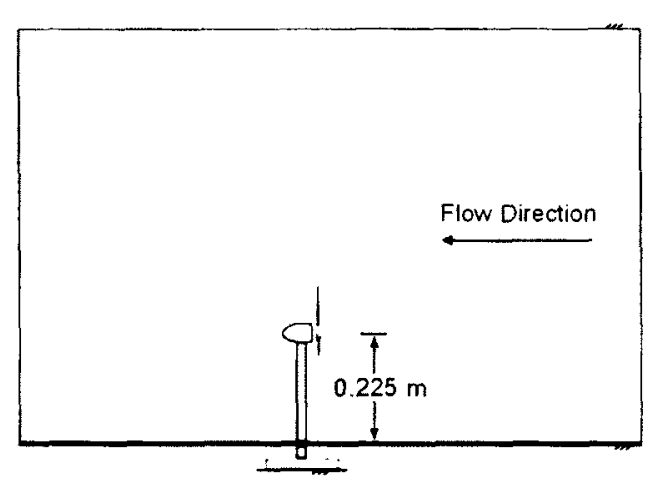

Side View of the Test Section

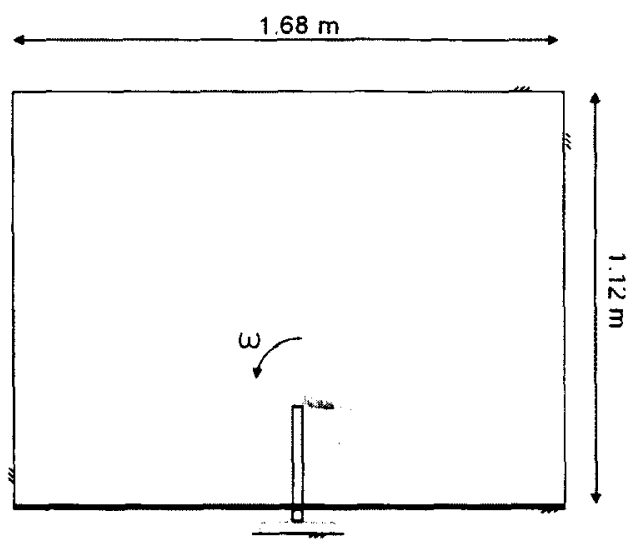

Front View

Figure 5.11: Schematic of the upstream test section in the ABL tunnel.

Dimensions are not to scale.

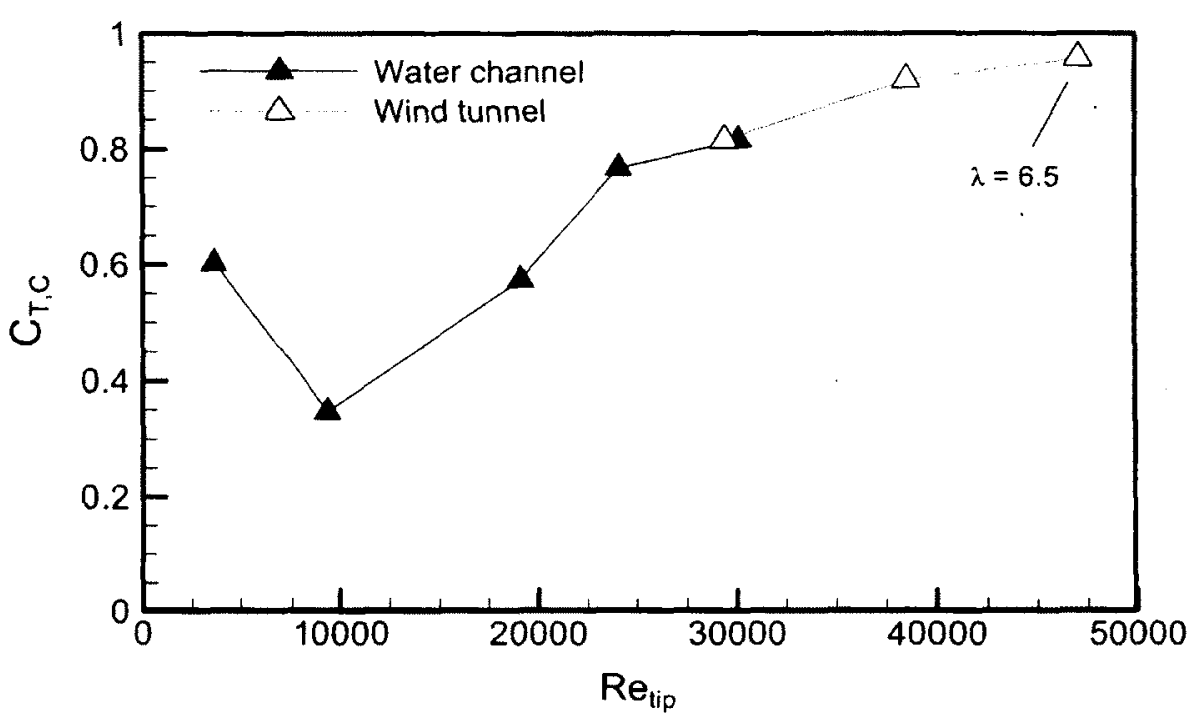

Figure 5.12: Comparison of wind tunnel and water channel-derived $C_{T, C}$ for the MAAE rotor at $\lambda \approx 6$.

\subsubsection{Scaled TU Delft thrust coefficients}

Blockage-corrected thrust coefficients were obtained from the recorded forces for the four tested configurations with the scaled TU Delft rotor. The blockage correction resulted in a maximum thrust coefficient correction of $6.1 \%$. The scaled rotor thrust coefficients presented in Figure 5.13 are seen to underpredict the medium-scale results. The lowest 
Reynolds number case had a thrust coefficient that was approximately $60 \%$ smaller than the corresponding medium-scale test. The difference between the scaled and mediumscale results, however, is reduced when the Reynolds number was approximately 30.000 . The observed reduction in all of the small-scale thrust coefficients is consistent with the reduced lift coefficients. reduced stall angles. and predominance of purely laminar separation associated with the NACA 0012 airfoil at Reynolds numbers in the range of 12.800 $31,400[148,166]$. Geometric scaling and matching $\lambda$ will result in a significantly lower thrust coefficient and an associated narrower wake expansion if the airfoil properties are sensitive to changes in Reynolds number. These results indicate that additional factors beyond geometric-scaling must be considered when conducting scaled tests at a low Reynolds number.

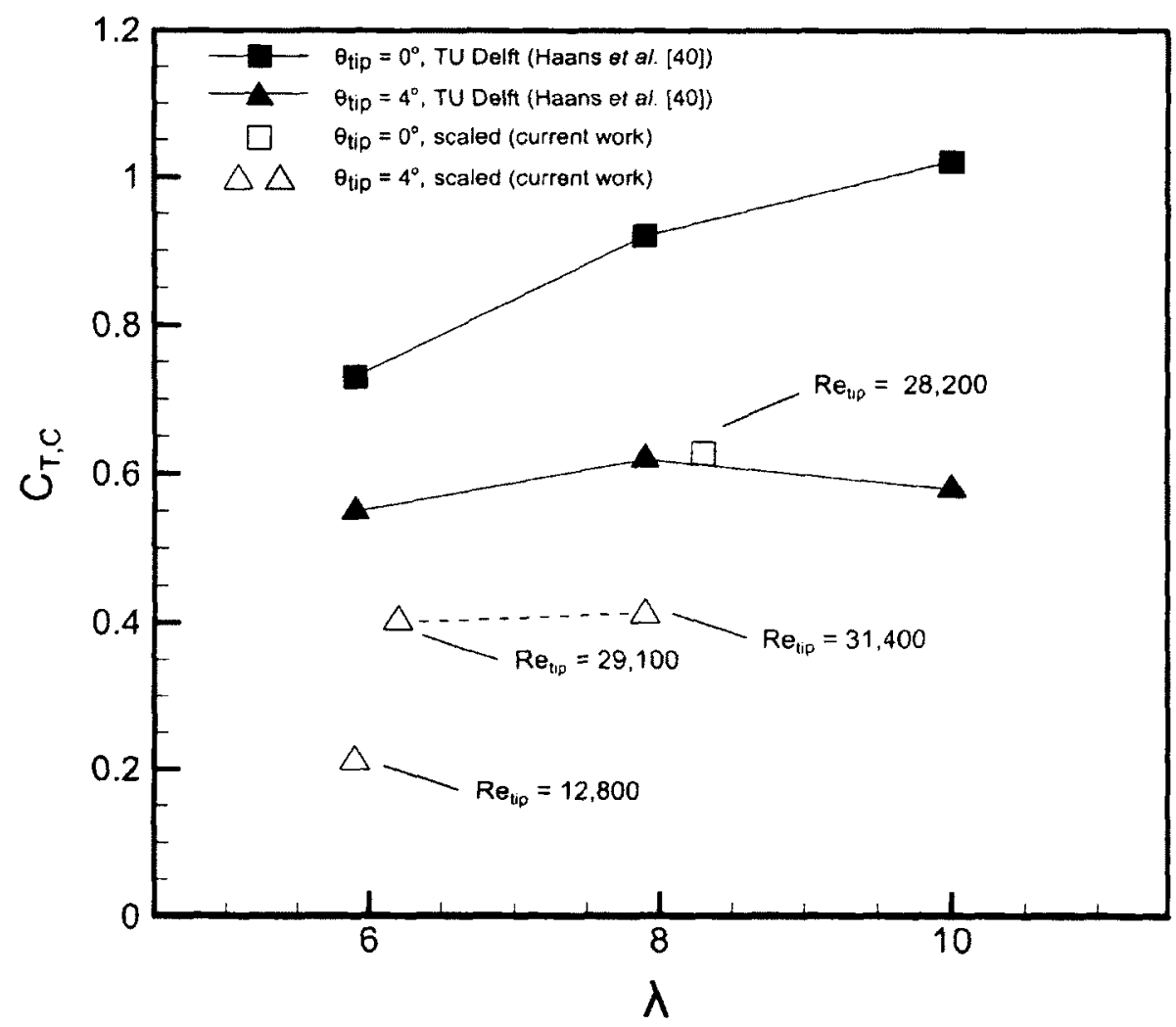

Figure 5.13: Reynolds number and tip pitch effects on $C_{T}$ with the scaled TU Delft rotor. 


\subsubsection{Comparison with other results}

The thrust coefficients generated by the MAAE rotor ranged from 0.77 to 0.92 in the practical Reynolds number regime of the scaled design (between 20.000 and 40.000). By comparison. the geometrically-scaled $\mathrm{TU}$ Delft rotor developed thrust coefficients that ranged from 0.21 and 0.63 and were $30 \%$ to $60 \%$ lower than the equivalent medium-scale results.

The thrust coefficient of a particular rotor will depend on its airfoil characteristics and its tip speed ratio. Although $C_{T}$ will also be dependent on $R e_{\text {tip }}$ for a particular turbine design, $C_{T}$ is not a function of $R e_{t i p}$ between different designs or scales of turbines. A stacked histogram of thrust coefficients for small-scale. medium-scale. full-scale. and commercialscale turbine designs has been compiled in Figure 5.14 from available results in literature in order to compare the current MAAE design to existing results.

The majority of the scaled turbine experiments have thrust coefficients that fall within bins ranging from 0.8 to 0.95 , similar to range of $C_{T}$ for the MAAE rotor. A number of small-scale experiments have thrust coefficients below 0.65 and many of these are static mesh or porous disc models. Although very little commercial-scale thrust information is available in literature, data for the $80 \mathrm{~m}$ Vestas V80, the $70.5 \mathrm{~m} \mathrm{GE} 1.5 \mathrm{~s}$, and the $35 \mathrm{~m}$ diameter Bonus $500 \mathrm{~kW}$ turbines were available in Refs. [10,99.170].

\subsection{Summary}

The current experiment investigated scaling effects in small-scale wind turbine experiments using two rotor models in a closed-loop water channel. A three-bladed rotor was designed specifically for testing in a low Reynolds number regime and a two-bladed rotor was geometrically-scaled from an existing. medium-scale design. The effect of changing the 


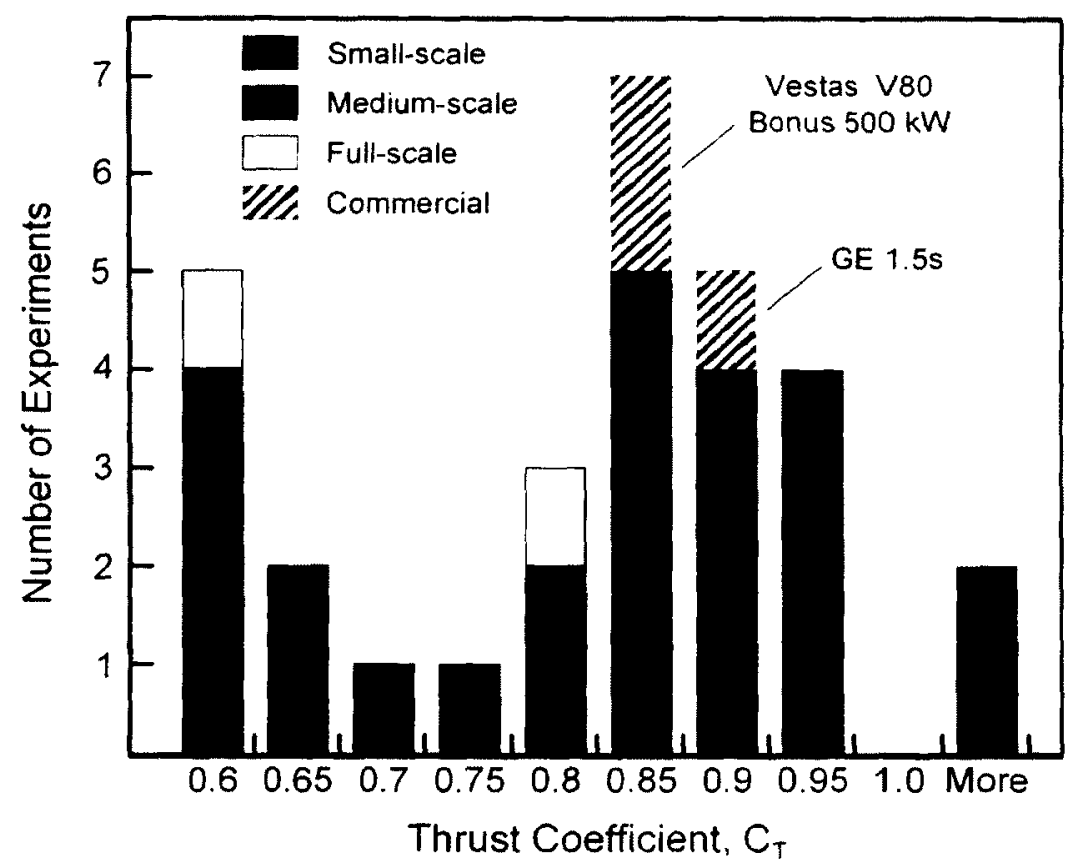

Figure 5.14: Compilation of $C_{T}$ values from experiments in literature.

With data from Refs. $[23.25,28-30,40.45,47.48 .51 .57 .59 .62 .77 .79,80.96,99,127,170.171]$.

Reynolds number on the initial wake expansion and the derived thrust coefficients associated with each rotor was assessed. The following results were identified as outcomes of this work:

- The wake expansion downstream of the three-bladed rotor increased with increasing Reynolds number and became less sensitive to changes above $R e_{t i p}=20,000$. The increase in wake expansion was attributed to an increase in the thrust coefficient with increasing Reynolds number. The wake expansion downstream of the geometricallyscaled two-bladed rotor was approximately $30 \%$ to $50 \%$ narrower than experimental results from the corresponding medium-scale experiment conducted at the Delft University of Technology. It was also observed that the tip vortex pitch was not strongly affected by Reynolds number. and was instead a function of the tip speed ratio and the lift distribution along a blade. which is characterised in this work by the tip pitch angle setting.

- The thrust coefficient was abserved to increase with increasing Reynolds number 
for the three-bladed MAAE rotor and approached the desired range of full-scale thrust coefficients. The thrust coefficient in the scaled TC Delft experiments was approximately $40 \%-60 \%$ lower than the corresponding medium-scale coefficient.

- Blockage corrections reduced the magnitude of the recorded thrust coefficients by up to $9 \%$ for the MAAE rotor and by up to $6 \%$ for the scaled TU Delft rotor. This confirms the recommendations developed in Chapter 4 that suggested blockage corrections may be beneficial even when blockage is approximately $10 \%$.

- The wake expansion and thrust coefficient of a small-scale model operating in a low Reynolds number regime may not match a scaled-up version of the same rotor unless the scaled rotor geometry or airfoil is modified. As a result. it is recommended that scaled wind turbine experiments should be conducted with rotors that have been tailored to the expected Reynolds number regime. Additionally, a minimum Reynolds number corresponding a reduced variation in the lift curve, transition, and separation behaviour should be reached in small-scale experiments. 


\section{Chapter 6}

\section{The performance of wind farms}

\section{with closely-spaced wind turbines}

The two largest losses in wind farm performance result from wake effects in grid-like wind farms and mean annual wind speeds that are lower than the rated capacities of turbines. Wake effects have been shown to result in downstream turbine efficiency losses of $5 \%$ to $40 \%$ depending on longitudinal separation distances and the wind direction [10]. The second source of losses can be quantified by investigating the annual capacity factor of a wind farm. recalled here as the energy actually generated by a wind farm compared to its rated energy capacity. The capacity factors of new, onshore wind farms in Ontario typically vary between $30 \%$ and $32.5 \%$. This occurs primarily as mean annual wind speeds are significantly lower than the wind speeds required for turbines to generate their maximum rated capacity. The ability to improve wind farm performance at mean annual wind speeds while reducing wake effects could therefore improve the annual capacity factor of wind farms.

The ability to improve the performance of wind farms was investigated using scaled wind farm layouts designed to capitalise on wake behaviour. The test conditions for the 
measurement campaign were identified using the guidelines established as a result of the blockage and Reynolds number campaigns. A parametric study of lateral and longitudinal wind turbine separation distances was conducted in an atmospheric boundary layer (ABL) wind tunnel and the effect of the lateral separation between three rotors on the power output and the wake development was assessed. This Chapter will describe the flow conditions developed in the atmospheric boundary layer wind tunnel and will then present the wind farm performance results in several steps. This involves first characterising the downstream flowfield and turbine behaviour of a single rotor. Typical losses due to wake effects in longitudinally-aligned wind farm configurations will then be verified using the scaled models. The in-field blockage effect identified in this work will then be presented using wake measurements downstream of several laterally-aligned wind turbine configurations. Lastly, the ability of the in-field blockage effect to increase the performance of wind farm configurations will be described.

\subsection{Atmospheric boundary layer characteristics}

Wind farm performance was assessed using scaled $20 \mathrm{~cm}$ diameter wind turbine models in the ABL wind tunnel at Carleton University. The ABL wind tunnel at Carleton University was commissioned in 1979 and several flow mapping studies were conducted between 1979 and 1982 [158]. Since that time, however, a second wind tunnel was installed in the room containing the $\mathrm{ABL}$ tunnel for blade vortex interaction (BVI) experiments and this additional tunnel creates a large blockage on one side of the open return portion of the ABL tunnel. Additionally, the roughness panels that were built for the wind farm experiments also differed from the original roughness panels that had been used in the 1980s. Given the change in the flow behaviour due to the BVI wind tunnel and the new roughness panels. the boundary layer developed in the current configuration was characterised prior to conducting 
the performance measurement campaign.

Rough and smooth boundary layer profiles were generated in the wind tunnel using the flow conditioning devices previously-described in Chapter 3. The rough boundary layer was generated using the $83.8 \mathrm{~cm} \times 12.7 \mathrm{~cm}$ spires followed by full-length roughness elements. The smooth boundary layer was generated using $80.0 \mathrm{~cm} \times 5.1 \mathrm{~cm}$ spires with roughness along only $25 \%$ of the fetch distance in the wind tunnel. The boundary layer measurements were all obtained using a single-normal hot-wire probe using the techniques identified earlier in Chapter 3. The smooth and rough boundary layer profiles at the tunnel centreline at the $X=0$ plane can be seen in Figure 6.1(a). Note that the upper-most data point in Figure 6.1 (a) was recorded using a pitot-static tube. The associated turbulence intensity profiles for the boundary layers at the tunnel centreline can be seen in Figure 6.1(b). The rough ABL approximates a power law distribution with $\alpha=0.25$ and has a $U_{\text {ref }}$ and a turbulence intensity at hub height of $5.8 \mathrm{~m} / \mathrm{s}$ and $13.2 \%$, respectively. The smooth boundary layer. however, approximates a power law distribution with $\alpha=0.1$, has a $U_{\text {ref }}$ of $5.9 \mathrm{~m} / \mathrm{s}$ and a hub height turbulence intensity of 5.5\%. The two boundary layers approximately represent commonly-assumed power law profiles in the atmosphere. Although the two boundary layers were designed to produce a similar wind turbine Reynolds number. the rough boundary layer was meant to mimic onshore partially-forested terrain and the smooth boundary layer more closely matches offshore conditions.

\subsubsection{Flow asymmetry}

Flow asymmetry in the test section was subsequently evaluated in the downstream test section of the ABL tunnel by recording a plane of data at the $X=0$ plane. Nine spanwise traverses were conducted in horizontal measurement intervals of approximately $40 \mathrm{~mm}$. The measurement window shown schematically in Figure 6.2 was restricted by the reachable 


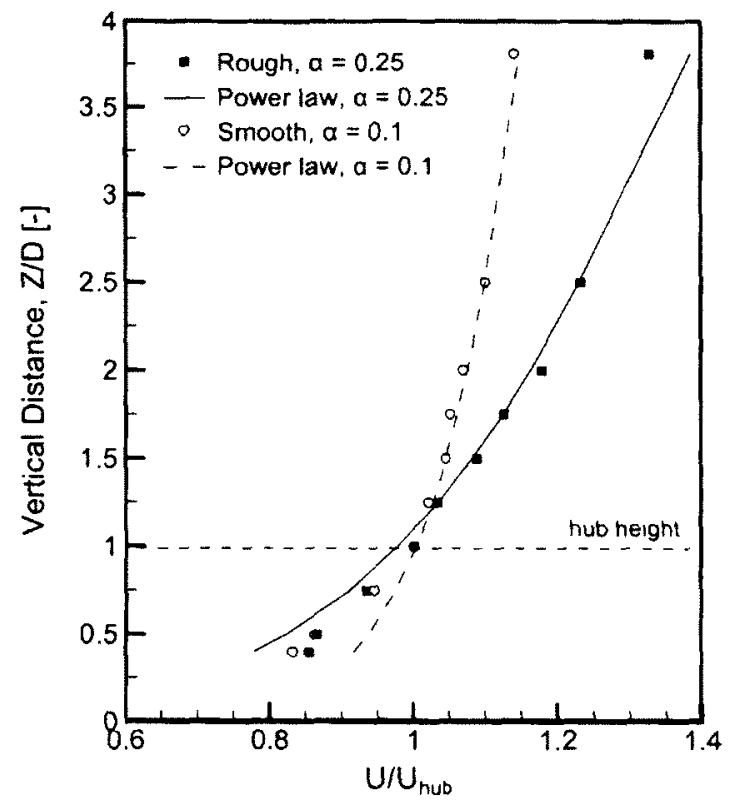

(a) Boundary layer velocity profiles.

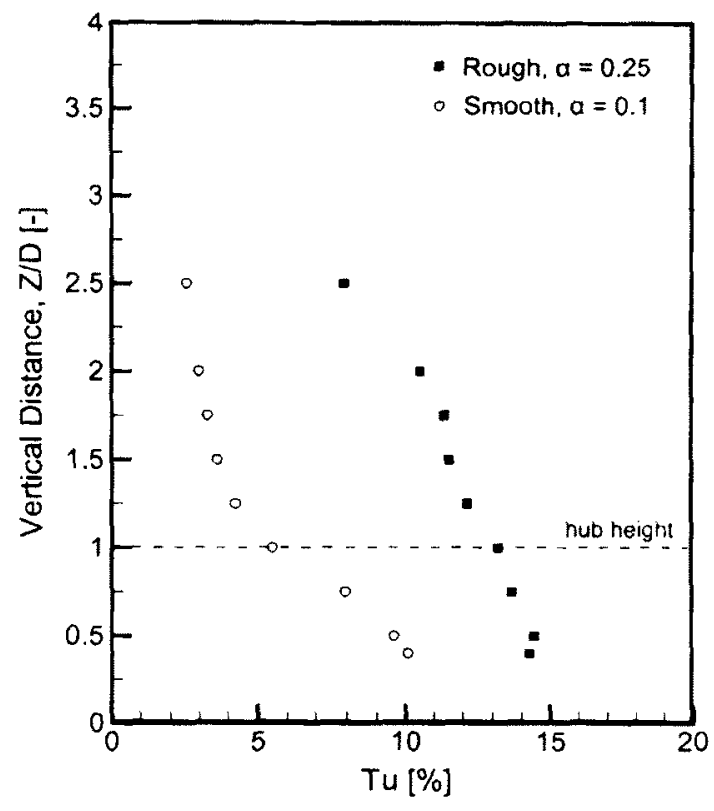

(b) Turbulence intensity profiles.

Figure 6.1: Velocity and turbulence intensity profiles at $X=0$ and the tunnel centreline.

workspace of the traverse and represents $20 \%$ of the cross-sectional area but encompasses the lateral range in which the wind turbines were installed. The speed variation in the $Y-Z$ plane with rough and smooth boundary layers can be seen in Figures 6.3(a) and 6.3(b). respectively. Dots indicating the measurement locations and shaded circles highlighting the outermost turbine positions are included in Figure 6.3. The contour plots identify that the centreline speed decreases slightly moving laterally away from the centreline. The effect of the observed variation in the speed magnitude at the turbine hub height was accounted for in the experimental campaign and is described in the next Section.

\subsection{Single turbine results}

The wake developed by a single turbine was evaluated in order to obtain a baseline condition for the flowfield, to verify the wake expansion with earlier results from Chapter 5 . and to validate the wake recovery characteristics with other results available in literature. 


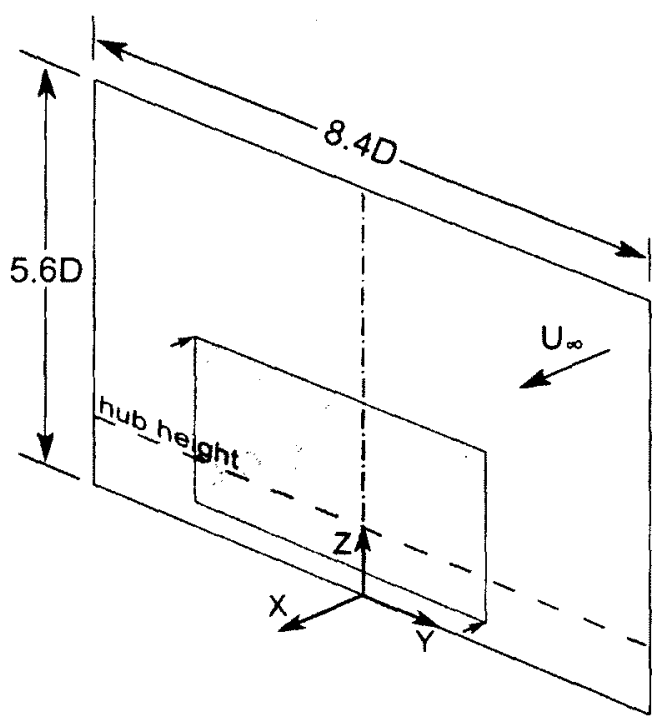

Figure 6.2: Schematic of the ABL measurement plane.

Following the characterisation of the flowfield, the position-dependence of the power output of a turbine was evaluated in order to account for the identified speed non-uniformities in the test section.

A $20 \mathrm{~cm}$ wind turbine was installed at the $X=0 . Y=0$ position, which is located at the upstream measurement plane along the tunnel centreline, and was operated with a tip speed ratio of approximately 6 and a Reynolds number of 38,000 , based on the tip conditions. The wind turbine was operating with a reference speed at hub height of 5.8 $\mathrm{m} / \mathrm{s}$, as established from the boundary layer measurements. Chapter 5 identified that the wake expansion behaviour of this three-bladed rotor became less sensitive to changes in the flow conditions above $R e_{t i p}=23.000$. The $R e_{t i p}$ of an isolated rotor was subsequently selected not only to be above this limit, but also so that turbines operating in the wake of an upstream turbine (and experiencing a lower inflow speed) will also be operating in a favourable Reynolds number regime. A series of horizontal traverses were conducted at the turbine hub height in the measurement window highlighted in Figure 6.4. The mean flowfield speed was calculated from the hot wire data and was normalised with respect to 


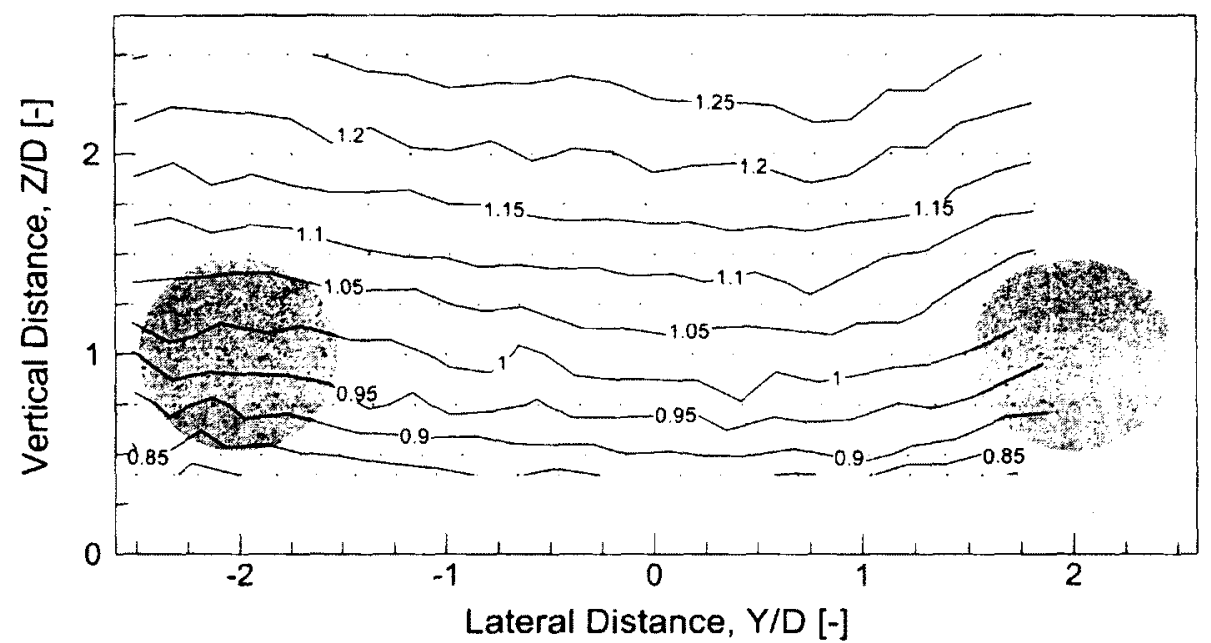

(a) Rough boundary layer.

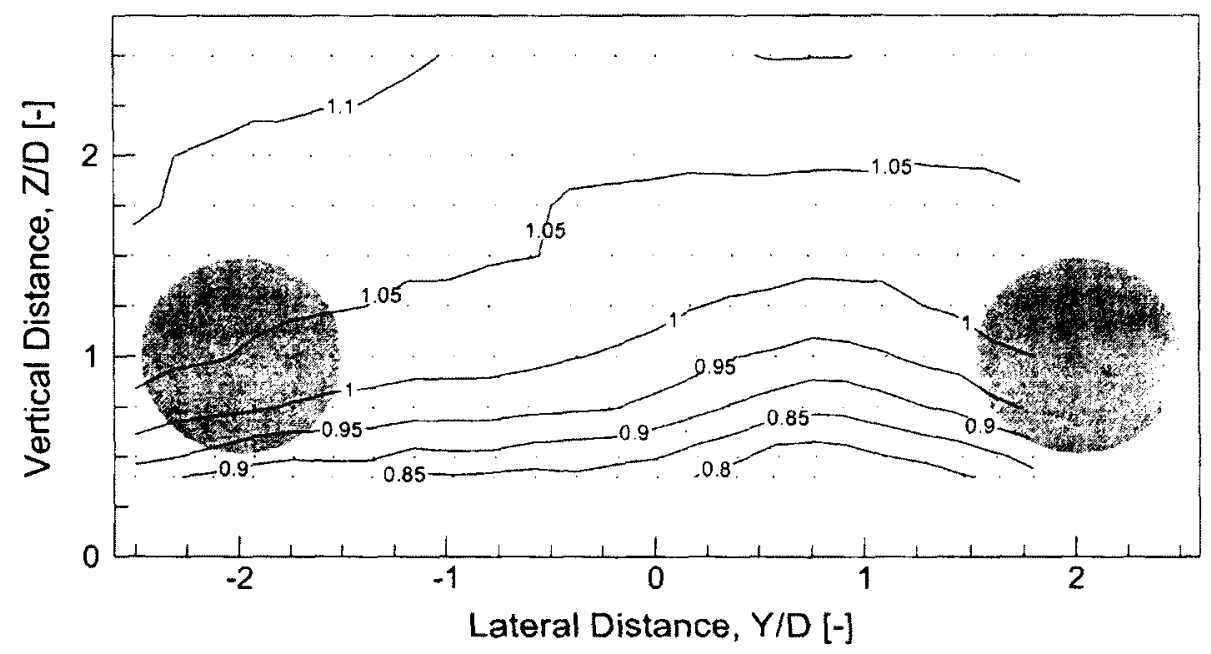

(b) Smooth boundary layer.

Figure 6.3: Normalised speed contours at $X / D=0$.

the hub height reference speed.

Mean speed contours at downstream planes ranging from $0.5 \mathrm{D}$ to $9 \mathrm{D}$ are shown in Figure 6.5. These contours, along with subsequent contour plots, were generated using Tecplot 360 . Note that the contour levels have been deliberately cut off below a normalised speed of 0.35 in order to reflect the limitations of the HWA calibration. It was mentioned in Chapter 3 that the calibration setup limited the minimum speed that could be reliably calculated to 


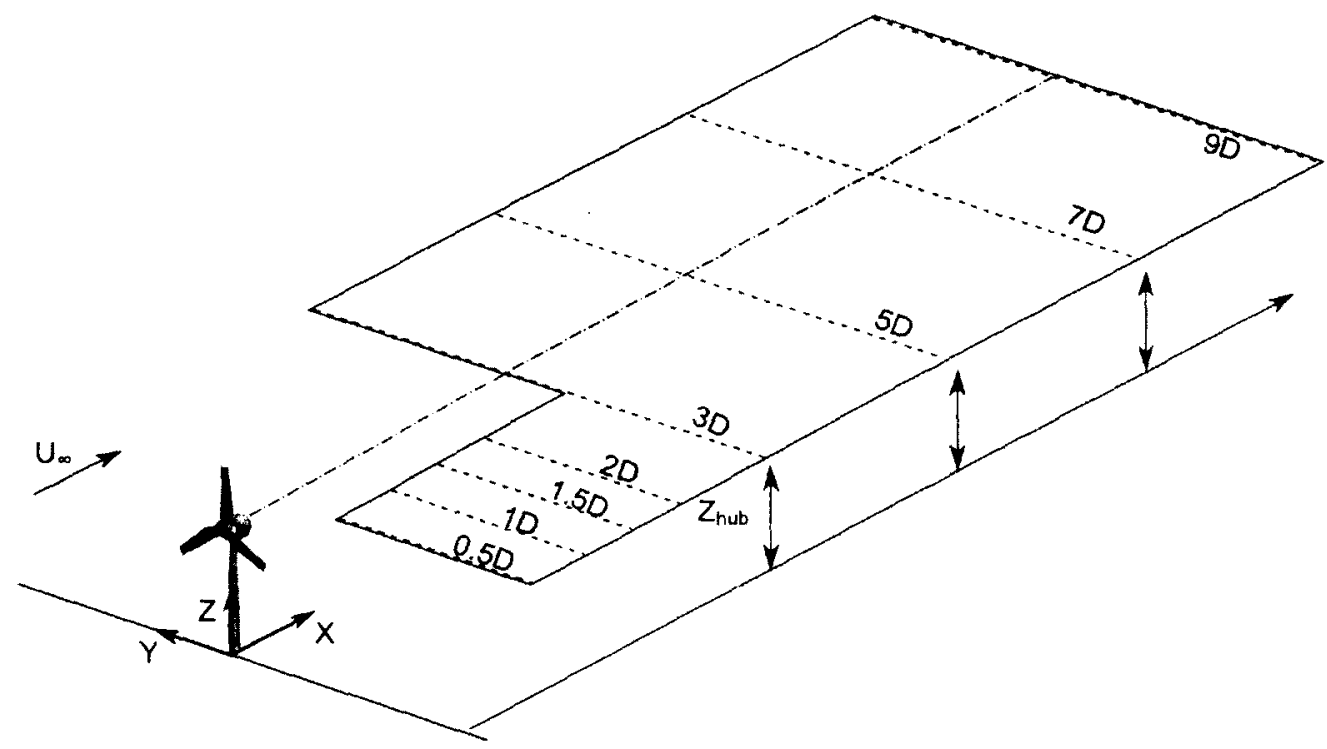

Figure 6.4: Schematic of the single rotor HWA measurement window.

$2 \mathrm{~m} / \mathrm{s}$, corresponding to $U / U_{l u b}=0.35$. Care was taken to avoid regions where the speed would be below this threshold along with those where rotation may be present in the wake (typically within 3D immediately behind the rotor). The data points where the speed was below $2 \mathrm{~m} / \mathrm{s}$ represented less than $1 \%$ of the speed data recorded in all of the considered test cases and so these points were subsequently omitted from the contour plots. Additionally, dots indicating all of the measurement points have been included in the contour plots in order to more clearly denote the measurement planes.

The mean speed contours demonstrate that the wake expands downstream of the turbine and recovers to $90-95 \%$ of the freestream speed 9D downstream. Labels have been included along the 1.00 contour lines, which represents where the speed is equal to the reference freestream speed at hub height. These 1.00 contour lines confirm that the speed returns to the freestream level outside the wake region.

The outline of the wake profile. corresponding approximately to the wake expansion defined in Chapters 4 and 5. was assessed using the reduced speed data by identifying the lateral distance in the wake where $U=U_{r e f}$ at each of the downstream planes. The wake 


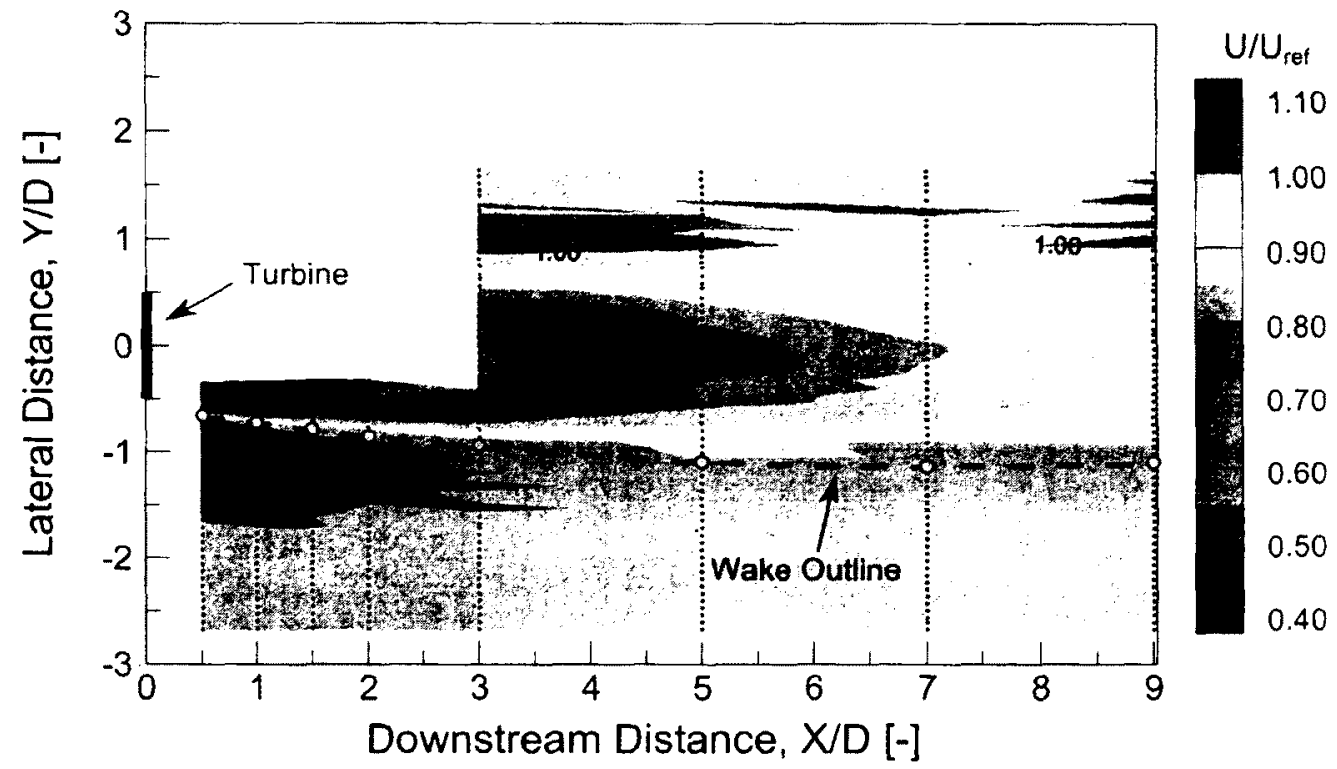

Figure 6.5: Hub height contour plot - Single rotor.

expansion profile determined using this approach is overlayed on the contour plot in Figure 6.5. The discrete data points used to highlight the wake expansion profile are represented with exaggerated hollow circles and have been connected for visualisation with straight. dashed, line segments. It had been previously assumed that the tip vortices could be used to represent the edge of the wake, but this only applies within $1 \mathrm{D}$ of the rotor while the discrete tip vortices were still visible. The approach used in this Chapter allows the wake profile to be identified further downstream. The consistency of the profile between the tip vortex visualisation conducted in the near wake region in Chapters 4 and 5 was verified with the far wake expansion profile determined with HWA traverses. The lateral positions of the wake profiles obtained using the two methods were calculated relative to the blade tip and are compared in Figure 6.6. The data points used to identify the far wake profile have been connected with straight line segments for visualisation. similar to the their presentation in Figure 6.5. The tip vortex-derived wake expansion profile was obtained from the earlier water channel experiments conducted at $R e_{t i p}=30.100$ when Blade 2 was passing through the dye. This differs from the wind tunnel test conditions with the $20 \mathrm{~cm}$ rotor which 
were set at $R e_{t i p}=38.000$. Despite the differences in the test conditions. a good degree of consistency can be observed by comparing the two data sets. Although the wake appears to contract slightly at the $X / D=9$ position. this difference falls within the uncertainty of the velocity data based on hot wire results. This uncertainty was assessed as approximately $\pm 0.5 \%$ of the mean speed and was based on quantization errors. temperature sensitivity. and calibration errors.

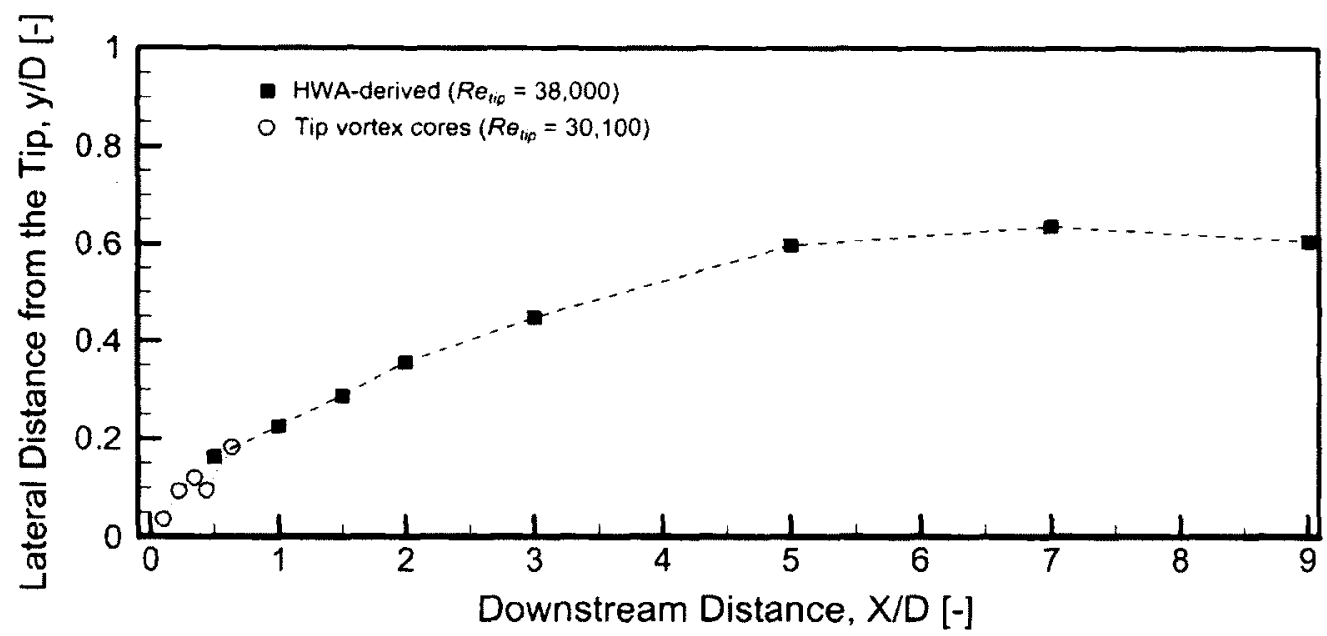

Figure 6.6: Comparison of the wake expansion profile for a single rotor.

Following the identification of the wake profile. the speed distribution at several downstream planes in the wake was validated with experimental data available in literature [99]. A scaled wind tunnel experiment conducted at the Energy Center of the Netherlands (ECN) used $25 \mathrm{~cm}$ diameter two-bladed turbines that had been scaled to operate with a Reynolds number above 25,000 and to generate a thrust coefficient representative of fullscale turbines $[79,99]$. The ECN turbine was operated with $C_{T}=0.91$ and speed profiles were recorded from $1 \mathrm{D}$ to $20 \mathrm{D}$ downstream of the turbine using a crosis-wire HWA [99]. The results in Chapter 5 indicated that the MAAE rotor generated a thrust coefficient of 0.92 at $\lambda=6.11$ and $R e_{t i p}=38.500$ and therefore the ECN experiment was selected for validation due to the similar thrust coefficient. The ECN experiment with a single rotor corresponded to $0.8 \%$ blockage whereas the current experiment had blockage of $1.67 \%$. Additionally. the 
turbulence intensity at hub height was $8.5 \%$ in the ECN experiment and $13 \%$ in the current experiment [78].

The lateral hub-height wake speed profiles inferred downstream of the MAAE rotor are compared to those from the ECN experiment in Figure 6.7 at several downstream planes. Available data from the ECN experiment at downstream locations of $1 \mathrm{D}, 2 \mathrm{D} .3 \mathrm{D}$. and $5 \mathrm{D}$ were directly compared to the results from the present work. Note that only every second data point is shown in the speed profiles for the current experiment and that the $x$-axis has been inverted. Good agreement can be seen between the two experiments and similar flow features are observed. The increase in speed above $U / U_{\text {ref }}=1$ near the blade tips at $1 \mathrm{D}$ and $2 \mathrm{D}$ is predicted well by the present experiment. Turbine hub effects are visible at $1 \mathrm{D}$ in the ECN data near the turbine centreline but such hub effects were not observed with the MAAE rotor. The Gaussian speed profiles at $3 \mathrm{D}$ and $5 \mathrm{D}$ also compare well with those from the ECN experiment. The ECN data set had a wake speed profile available at $8 \mathrm{D}$ but traverses were conducted in the current experiment at $7 \mathrm{D}$ and $9 \mathrm{D}$. The three traverses are therefore compared in the right side of Figure 6.7 and it can be observed that the wake profiles at $7 \mathrm{D}$ and $9 \mathrm{D}$ bracket the available $\mathrm{ECN}$ data at $8 \mathrm{D}$. This validation indicates that the wake width. flow speed profile, and downstream wake recovery are all being well characterised with the current turbine and ABL configuration.

The final aspect of the single rotor test that was characterised was the positiondependence of the power output. The speed variation in the test section that was observed in the previous Section would lead to the situation where a single isolated turbine would generate more (or less) power depending on its position in the wind tunnel test section and this behaviour would make the interpretation of multi-rotor performance quite challenging. This variation was therefore accounted for by recording the power output of a single undisturbed rotor at several of the possible turbine positions in the tunnel. The isolated rotor data was subsequently used to normalize a turbine s power output based on its position 

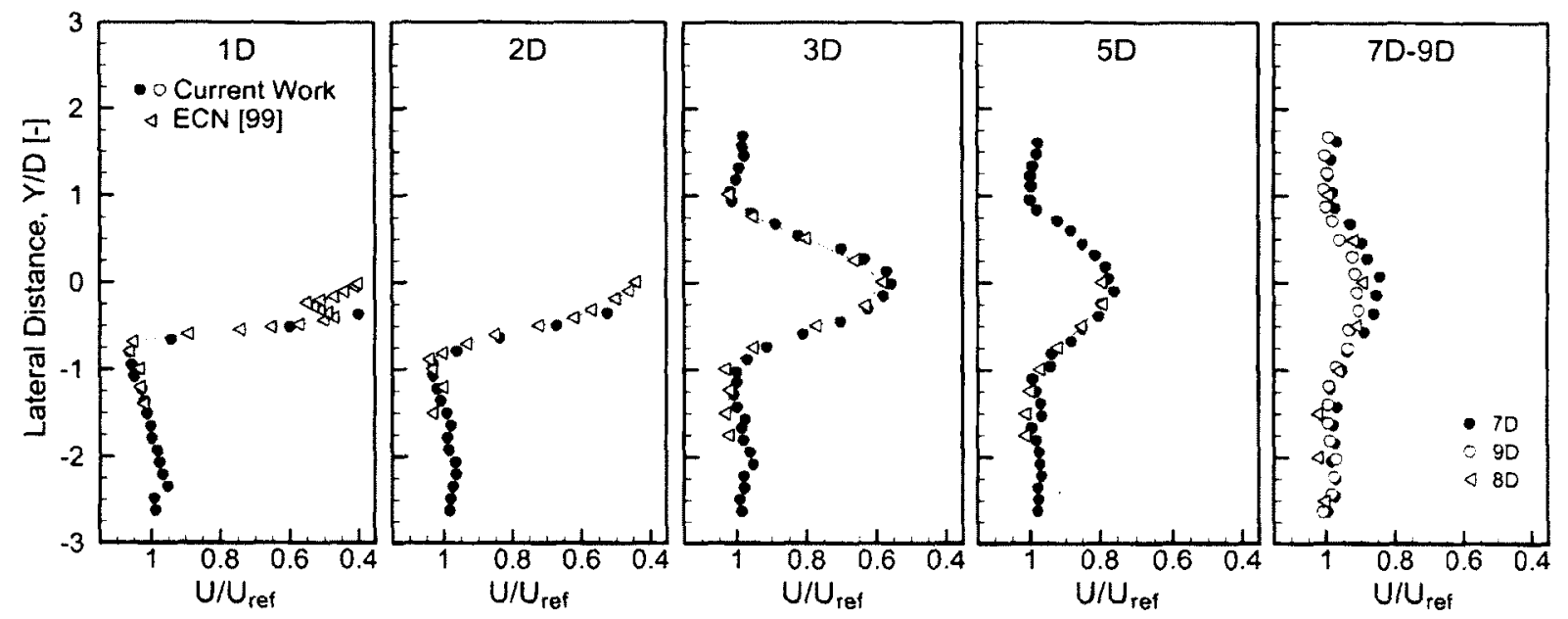

Figure 6.7: Wake speed validation downstream of a single rotor.

in the wind tunnel.

The effect of the variation of the wind speed within the tunnel on the power output of an individual turbine is presented in Figure 6.8. The wind tunnel speed was set in each case using a Pitot-static tube located along the tunnel centreline, $76 \mathrm{~cm}$ above the tunnel floor, as a reference. The variability of the power output in the lateral direction shown in Figure 6.8(a) indicates that the wind speed is reduced away from the centreline of the tunnel and this is consistent with the behaviour noted in the flow asymmetry measurements. The outermost turbine positions result in a tip clearance distance of $1.5 \mathrm{D}$ between the blade tip and the tunnel wall $(30 \mathrm{~cm})$. The longitudinal variation for a single rotor along the test section centreline is shown in Figure 6.8(b). The power output of the rotor in a rough boundary layer was the highest when located either at the upstream position or further downstream in the test section. Note that the turbine position located 2.5D upstream of $X=0$ in Figure 6.8(b) was only used for the test case with two longitudinally-aligned rotors. A position of $X=0$ corresponds to the lateral measurement plane and is located $10 \mathrm{~cm}$ downstream of the beginning of the downstream test section.

The wind turbine angular speed in these isolated rotor tests had an average standard 
deviation that represented $3.5 \%$ of the mean turbine rpm. This was a result of the velocity gradient in the boundary layer and the design of the turbines, which incorporated a DC generator and a potentiometer to set the target rpm. The resistance was maintained at a constant value and therefore the wind turbine rpm was allowed to fluctuate. The reference wind speed measured with a pitot-static tube in the single rotor tests had a mean speed of $7.96 \pm 0.06 \mathrm{~m} / \mathrm{s}$, which represents a maximum variation of $0.75 \%$ between tests.

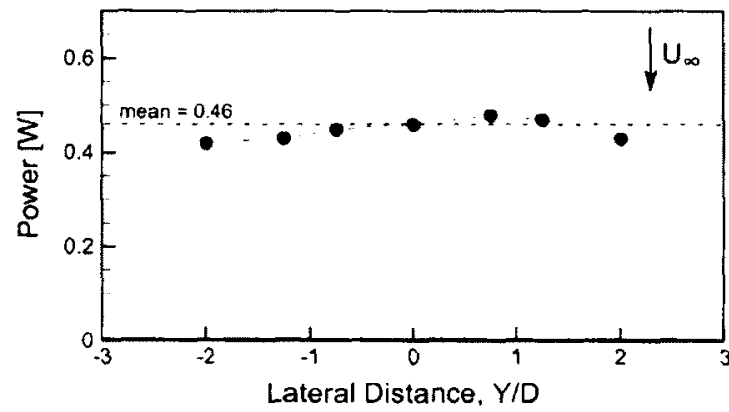

(a) Lateral variation in the test section.

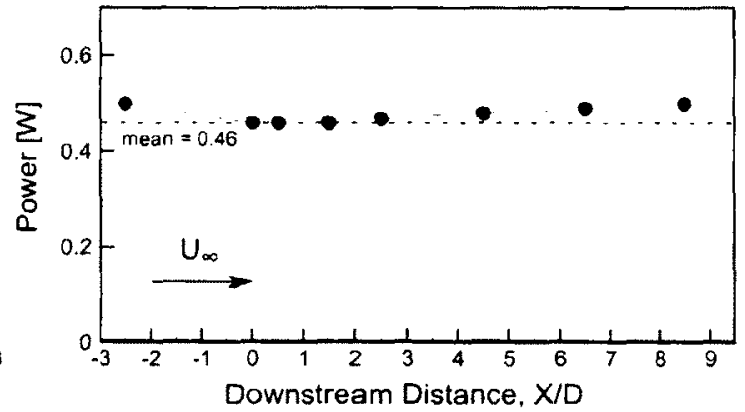

(b) Longitudinal variation along the tunnel centreline.

Figure 6.8: The power output of a single rotor at several wind tunnel positions in a rough boundary layer.

The power measurements in the multi-rotor configurations are normalized with respect to the single rotor data in order to account for the non-uniformities present within the test section. The subsequent power measurements $\left(P_{\text {rreas }}\right)$ will be presented as the change in the power output of a turbine relative to the power recorded at that location in the single, undisturbed, rotor case $\left(P_{\text {single }}\right)$ based on Equation (6.1). Similar position-correction approaches have been applied in Refs. $[77,79]$ in small-scale wind farm experiments. Ozbay assessed the rotational speed of a turbine located in the middle of the last row in a gridaligned wind farm configuration and compared the rpm to the rotor characteristics in isolated conditions at the same location in the tunnel [77].

$$
\Delta P=\frac{P_{\text {meas }}-P_{\text {single }}}{P_{\text {single }}} \times 100
$$




\subsection{Wake losses in longitudinal configurations}

A number of wind farms have a regular. grid-like design in which power losses due to the longitudinal alignment of turbines can be significant. The power output from turbines in the second row of a wind farm with downstream separation of $7 \mathrm{D}$ to $10 \mathrm{D}$ has been shown to be $40 \%$ to $20 \%$ lower, respectively, than the power output of the upstream rotors [10].

The current scaled models were designed with a thrust coefficient representative of fullscale turbines in order to generate an appropriate velocity deficit in the wake and the scaled $\mathrm{ABL}$ was generated to approximate the wake recovery distance. The wake recovery downstream of a single rotor was well validated with existing data from literature in the previous Section. This validation is extended to multi-turbine experiments by determining the power output of two longitudinally-aligned turbines at various separation distances. Two rotors were aligned in the longitudinal direction according to the schematic in Figure 6.9 and the downstream separation distance was varied from $5 \mathrm{D}$ to $11 \mathrm{D}$.

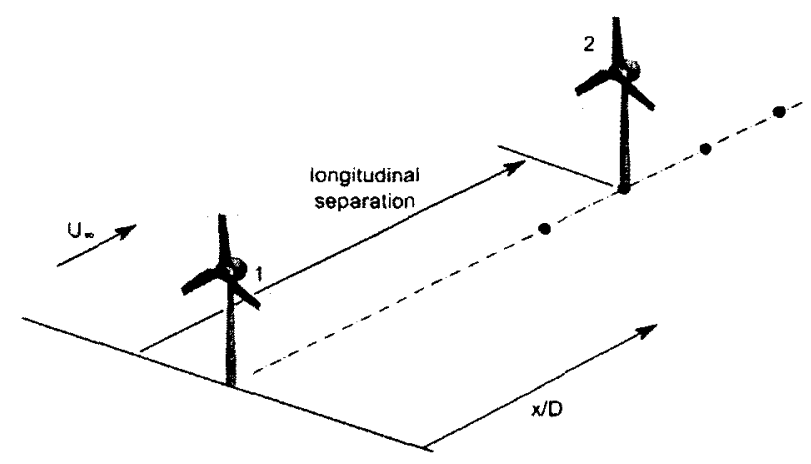

Figure 6.9: Longitudinally-aligned wind turbines.

The power output of the downstream rotor relative to the upstream rotor with respect to the downstream separation distance can be seen in Figure 6.10. The dotted line in Figure 6.10 is for representation only. A downstream separation distance of 5D yields a reduction in power of $40 \%$ and separation distances that range from $7 \mathrm{D}$ to $11 \mathrm{D}$ result in power deficits of $30 \%$ to $25 \%$. 


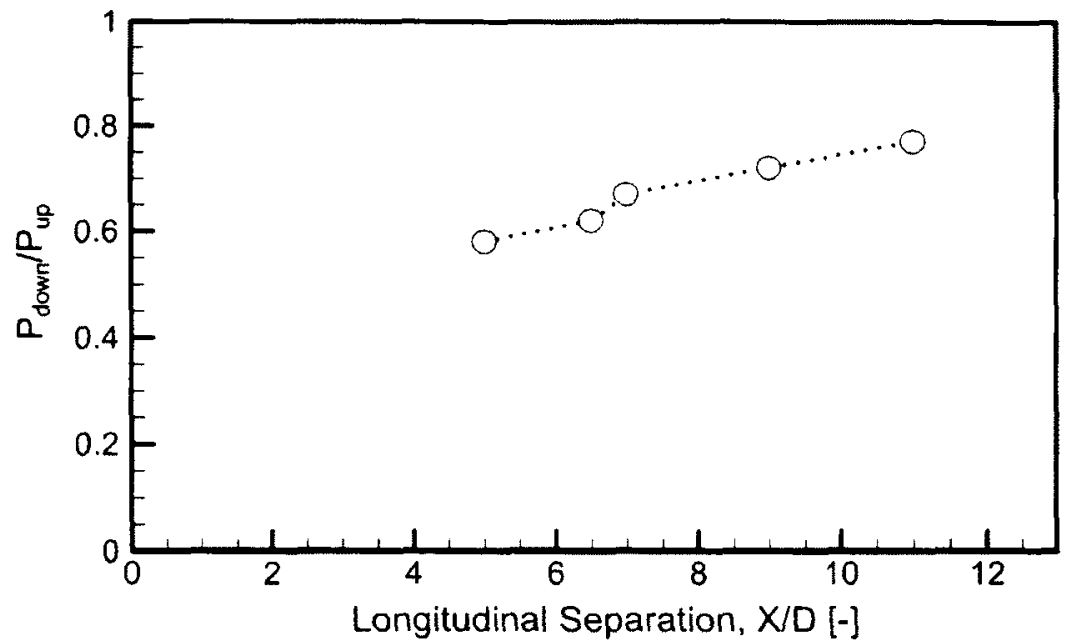

Figure 6.10: The power output of a downstream longitudinally-separated rotor.

The longitudinal separation results are compared to larger-sale experiments and fullscale results in Figure 6.11. The trends in the loss in power exhibited by longitudinallyaligned turbines is consistent with the larger-scale experiments conducted by Tsalicoglou et al. and Adaramola and Krogstad $[23,67]$. The results described by Adaramola and Krogstad were conducted with a thrust coefficient of 1.04 and tested the effect of the tip pitch angle $(\beta)$ of the upstream turbine on the power generated by the downstream turbine. Tsalicoglou et al. tested two turbines with $6.5 \mathrm{D}$ separation in a water tow tank. but the thrust coefficient was not reported in Ref. [67]. The power deficits highlighted in Figure 6.11 are also consistent with the full-scale wind farm performance results from the Horns Rev I wind farm published in Ref. [10], where a power deficit of $35 \%$ was observed with a 7D downstream separation distance between longitudinally-aligned wind turbines. Despite the similarity of the data in Figure 6.11 , a number of factors introduce uncertainties into the comparison, including the method of control of the rotors. the scale of the rotors. the ambient turbulence intensity of the various experiments, and varying amounts of blockage in each experiment. The trends in Figure 6.11, however, indicate that the scaled MAAE rotor generates a wake that has representative characteristics of larger experimental models as well as full-scale turbines. 


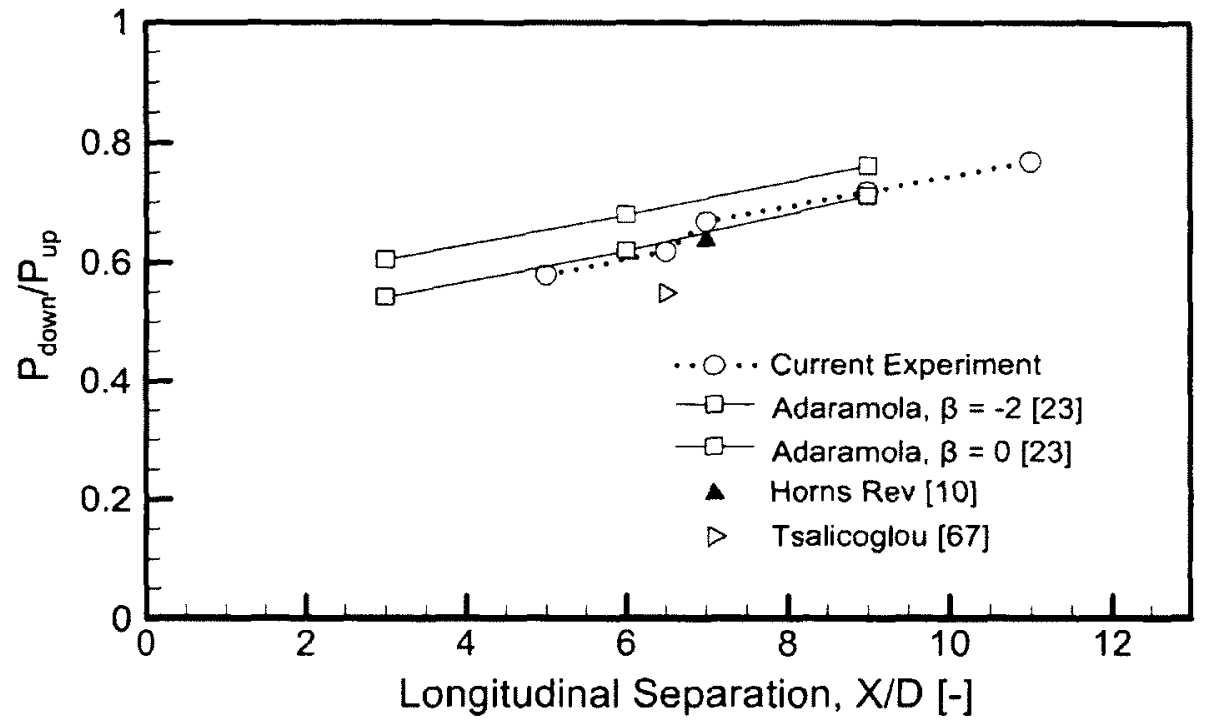

Figure 6.11: Comparison of longitudinally-aligned results.

The wake generated by a rotor was qualitatively shown to replicate the downstream wake behaviour of larger-scale models based on the performance of two longitudinallyaligned turbines. Grid-aligned wind farms, such as the Horns Rev I wind farm presented in Chapter 1, also exhibit an equilibrium-type behaviour in the wake, where the $3^{r d}$ and $4^{\text {th }}$ row turbines will generate approximately as much power as the $2^{\text {nd }}$ row turbines. The ability of the scaled turbines and boundary layer configuration to mimic this behaviour was assessed using the grid-aligned $3 \times 3$ wind farm shown in Figure 6.12(a). The scaled wind farm at Carleton University has 2D separation between rotor hubs in the lateral direction and $5 \mathrm{D}$ separation in the longitudinal direction. as shown in Figure 6.12(b). While these spacing distances are more compact than those observed in full-scale wind farms. other scaled wind farm experiments have used similar longitudinal separation distances due to the space constraints in typical $A B L$ test sections $[75,77,79]$.

The wind farm was tested in the rough $A B L$ and the power output of each turbine was calculated based on the recorded voltage output from the generator and its associated resistor. The power output of the turbines in the $3 \times 3$ wind farm was not position-corrected 


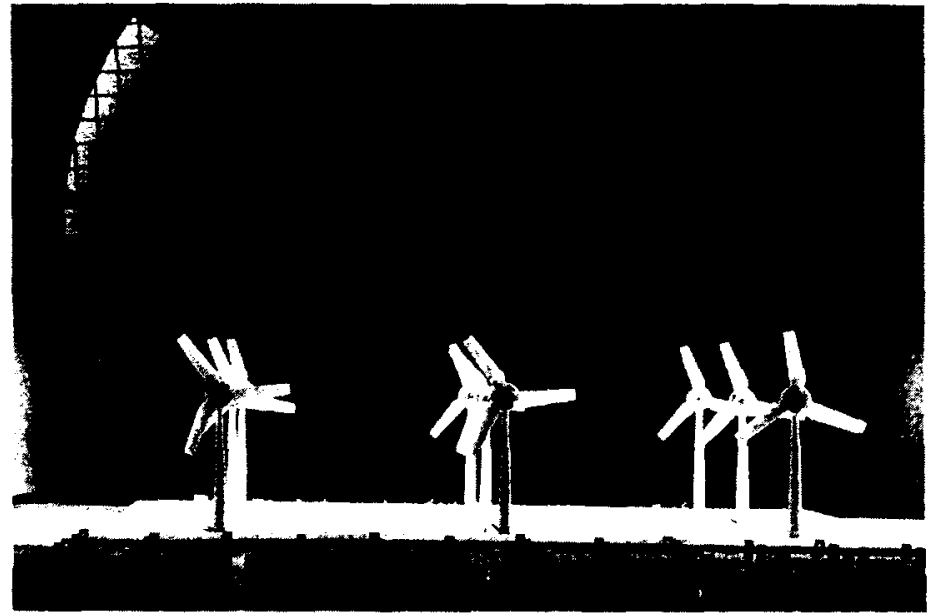

(a) Downstream view of the $3 \times 3$ wind farm

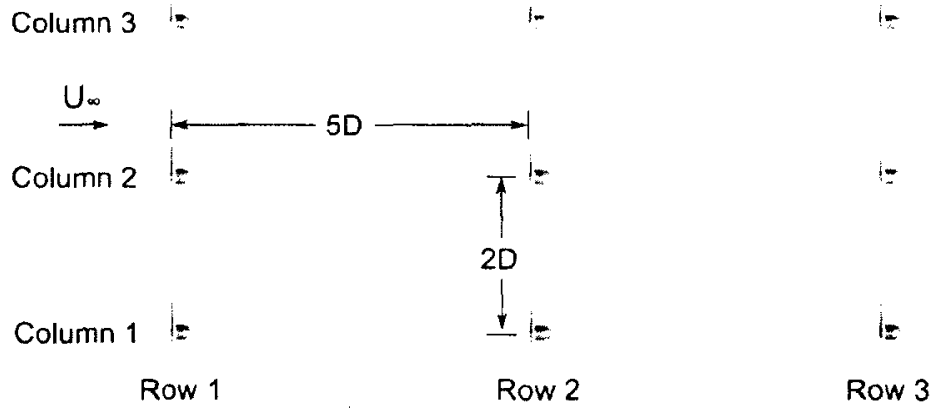

(b) Schematic of the $3 \times 3$ wind farm

Figure 6.12: The longitudinally-aligned $3 \times 3$ wind farm.

and therefore the output of the downstream turbines has been normalised by the first turbine in each column. The relative power drop for the three rows of turbines due to wake effects is shown in Figure 6.13.

The small difference in the power output observed between the $2^{\text {nd }}$ and $3^{\text {rd }}$ rows is consistent with the behaviour observed in other scaled wind farms as well as in full-scale measurements. The power output of the turbines in the middle column of the scaled wind farm is compared to the power output from turbines in the first four rows of the Horns Rev I wind farm and the output from the small-scale wind farm experiments conducted at the ECN in Figure $6.14[10,79]$. The $8 \times 3$ wind farm used in the ECN experiment had 3D lateral separation and $5 \mathrm{D}$ longitudinal separation in the upstream rows of the scaled farm. 


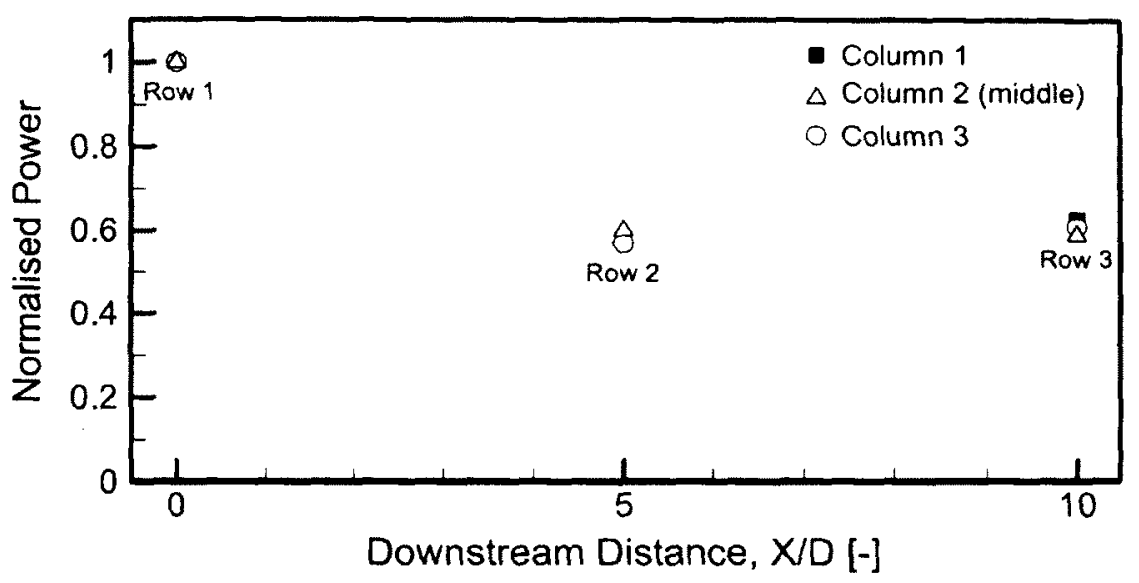

Figure 6.13: Power results in the $3 \times 3$ wind farm.

The Horns Rev I wind farm. however, has 7D spacing between turbines in the longitudinal direction. The results in Figure 6.14 qualitatively follow similar trends and the equilibrium in the relative power output between the $2^{\text {nd }}$ and $3^{\text {rd }}$ rows is consistent in all three cases. Other scaled wind farms described by Chamorro and Porté-Agel and by Ozbay exhibit a more gradual decrease in the power output as a function of row number [75,77]. This more gradual power decrease can be attributed to a low thrust coefficient of 0.17 in Ref. [77]. but a thrust coefficient is not reported by Chamorro and Porté-Agel in Ref. [75] or in their related works.

\subsection{Generating in-field blockage with closely-spaced rotors}

The wind farm test conducted with the scaled $3 \times 3$ farm, along with other experiments and full-scale measurements available in literature, have highlighted the fact that wake losses can be quite significant in grid-aligned wind farms. An alternative to both grid-like and irregular wind farms is presented in this thesis as a way to reduce the losses generated by downstream wake effects while capitalising on the behaviour of the wake itself to increase the energy yield of a wind farm. The observations in Chapter 4 that blockage both narrowed 


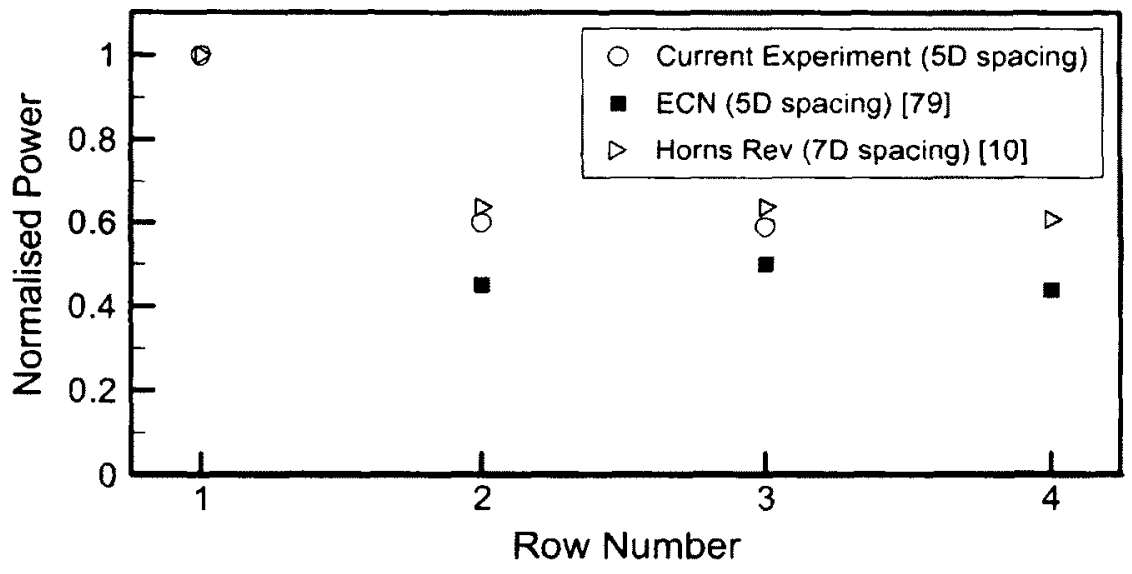

Figure 6.14: Comparison of wind farm results.

the downstream wake and resulted in higher forces have been applied to evaluate situations where turbines are spaced closely enough to generate a synthetic in-field blockage effect.

Previous work in Chapter 4 demonstrated that the initial wake expansion will not be strongly-affected by the tunnel walls when blockage is less than $10 \%$. Wind tunnel blockage calculated based a single rotor in the ABL tunnel was $1.67 \%$ and blockage based on the combined swept area of three laterally-aligned rotors was $5 \%$. The effect of this low level of blockage on recorded parameters will be minimal. The multi-rotor experiment was sized this way so that blockage effects (in the context of a rotor in a closed test section, as described in Chapter 4) could effectively be neglected. It was desirable to meet this condition so that the in-field blockage phenomenon generated by rotors in close lateral proximity could be studied in isolation of typical blockage effects.

In order to illustrate the in-field blockage effect, two $20 \mathrm{~cm}$ diameter rotors were placed at the $X=0$ plane in the wind tunnel test section and were separated by a gap $(G)$ according 
to Figure 6.15. Horizontal traverses with the HWA were conducted at the turbine hub height between $0 \mathrm{D}$ and $9 \mathrm{D}$ downstream of the rotor plane. Approximately 10-30 data points were recorded in the turbine gap region and 65 data points were recorded along the 3D-9D planes. This measurement process was repeated for gap distances of $3 \mathrm{D}, 2 \mathrm{D}, 1.5 \mathrm{D}$. and $0.5 \mathrm{D}$.

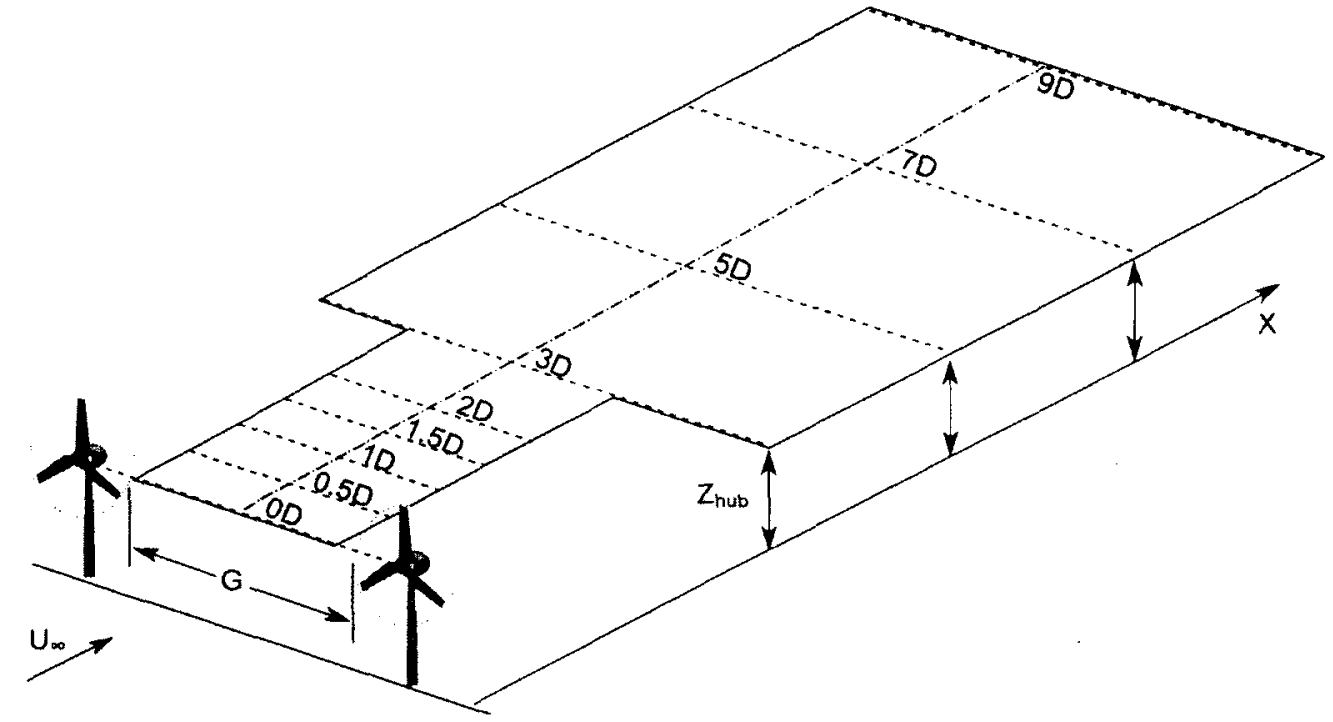

Figure 6.15: Schematic of the two-rotor arrangement and the HWA measurement plane. 
Contour plots of the normalised speed magnitude at hub height for each of the gap widths are presented in Figure 6.16. As in the single turbine case. the speed magnitude calculated from the HWA data was normalised with respect to the reference hub height speed (5.8 $\mathrm{m} / \mathrm{s}$ ) in order to clearly denote regions of increased and decreased speed. Contour levels below a normalised speed of 0.35 were omitted in a similar manner to the single turbine experiment to reflect the limitation on the minimum calibrated speed.

The normalised speed contours when $G=3 D$ in Figure 6.16(a) highlight that a region of increased speed occurs between the rotors. The region where the speed is increased by $4 \%$ to $7 \%$ compared to the freestream value extends from the upstream plane to $3 \mathrm{D}$ downstream. Labels have also been included to denote the 1.00 contour line. where the speed is equal to the freestream value. When the gap is narrowed to $2 \mathrm{D}$, a significant increase in speed can be observed in Figure 6.16(b) between $0.5 \mathrm{D}$ and $2 \mathrm{D}$ downstream and a core of higher speed extends all the way to 9D downstream. In this case, the maximum speed increases by more than $6-10 \%$ of the reference value.

Similar behaviour is noted in Figure 6.16(c) near the 1D plane, where the speed in the gap is increased by up to $11 \%$. In addition to the region of higher speed between the rotors. the wake profile in the near wake region between Turbines 1 and 3 appears to be narrowed from the $0.5 \mathrm{D}$ plane to the $2 \mathrm{D}$ plane. Figure $6.16(\mathrm{c})$ also demonstrates that the wind speed retains its freestream value outside the wake of Turbine 1, near the lower edge of the contour plot. This confirms the earlier claim that blockage (as a result of the tunnel walls) would not affect the flow development away from the rotors. The speed contours resulting from a $0.5 \mathrm{D}$ gap are visible in Figure $6.16(\mathrm{~d})$ and highlight a very small region between the blade tips where the wind speed is increased. a narrowing of the inner edge of the wakes. and a return to the freestream speed on either outer edge of the wakes. 

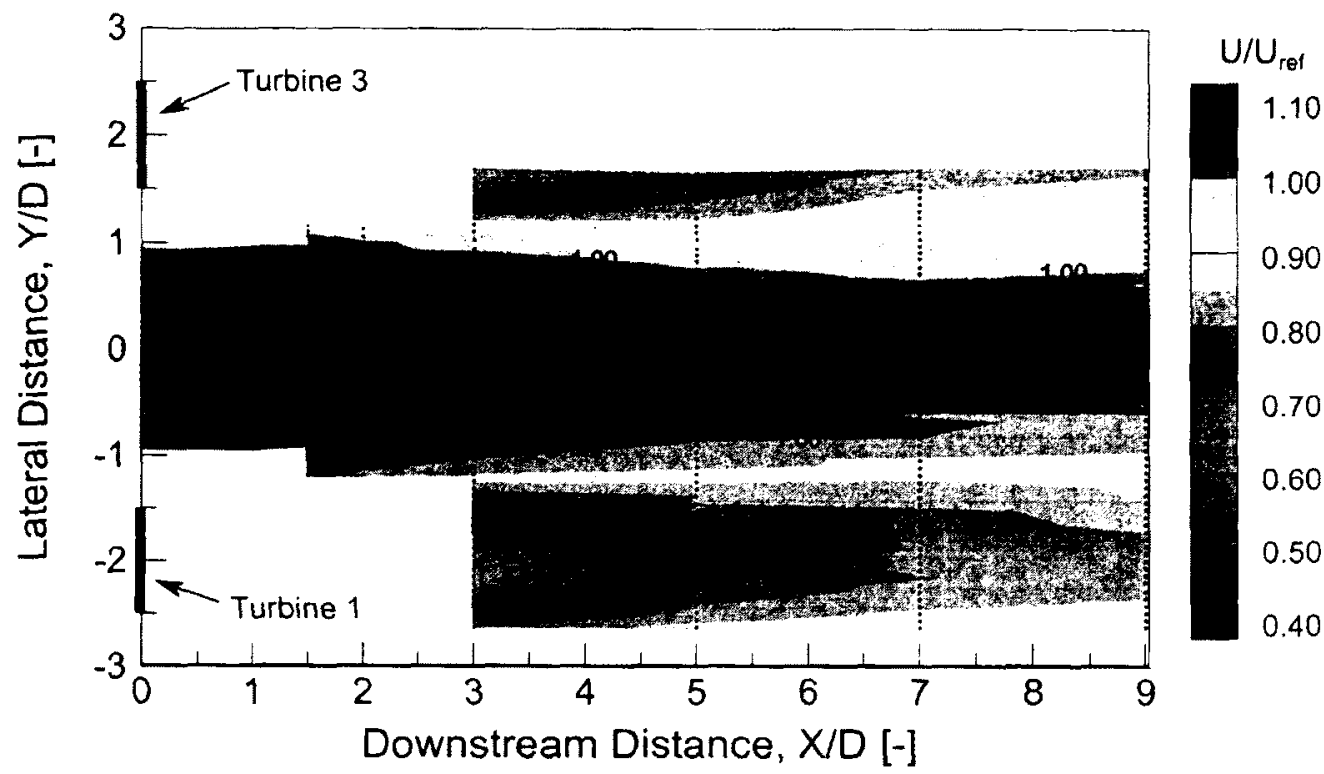

(a) Normalised velocity contours at hub height - 3D gap.

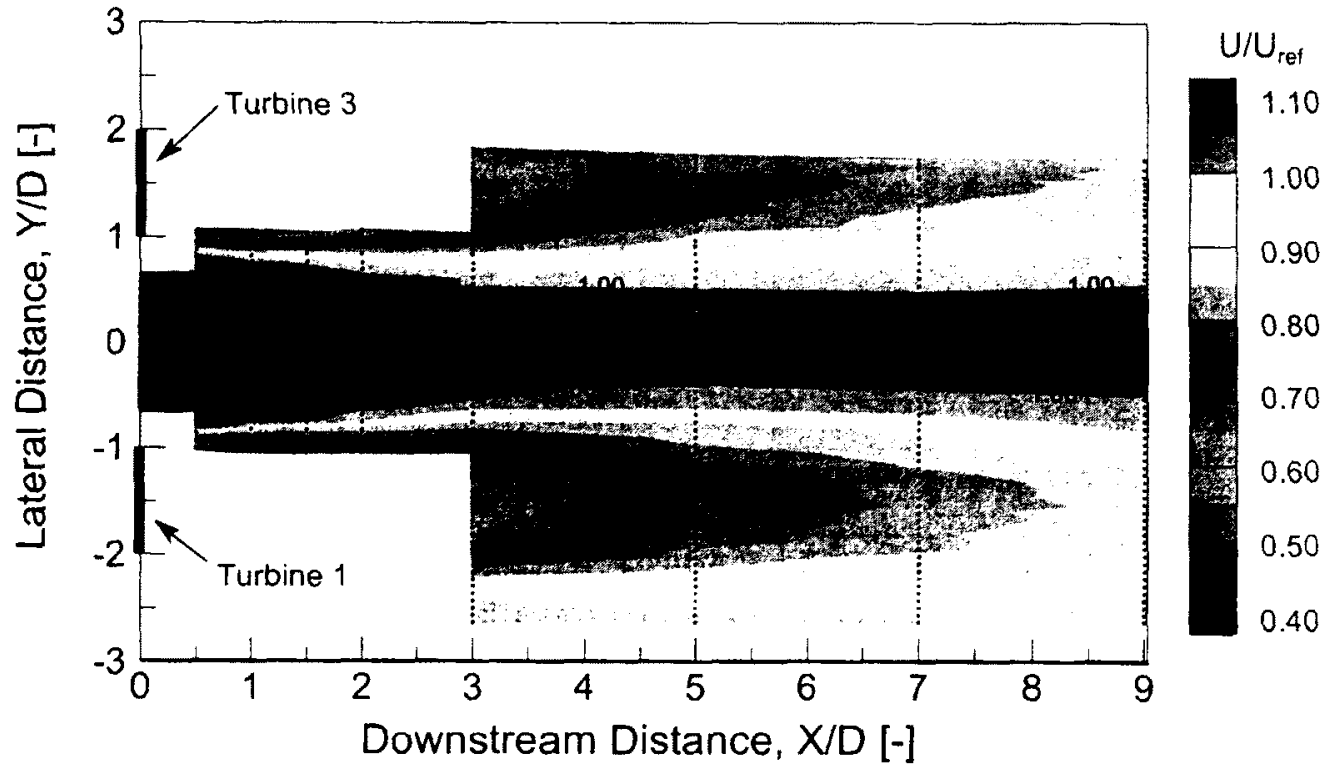

(b) Normalised velocity contours at hub height $-2 \mathrm{D}$ gap.

Figure 6.16: Normalised speed contours at hub height. 


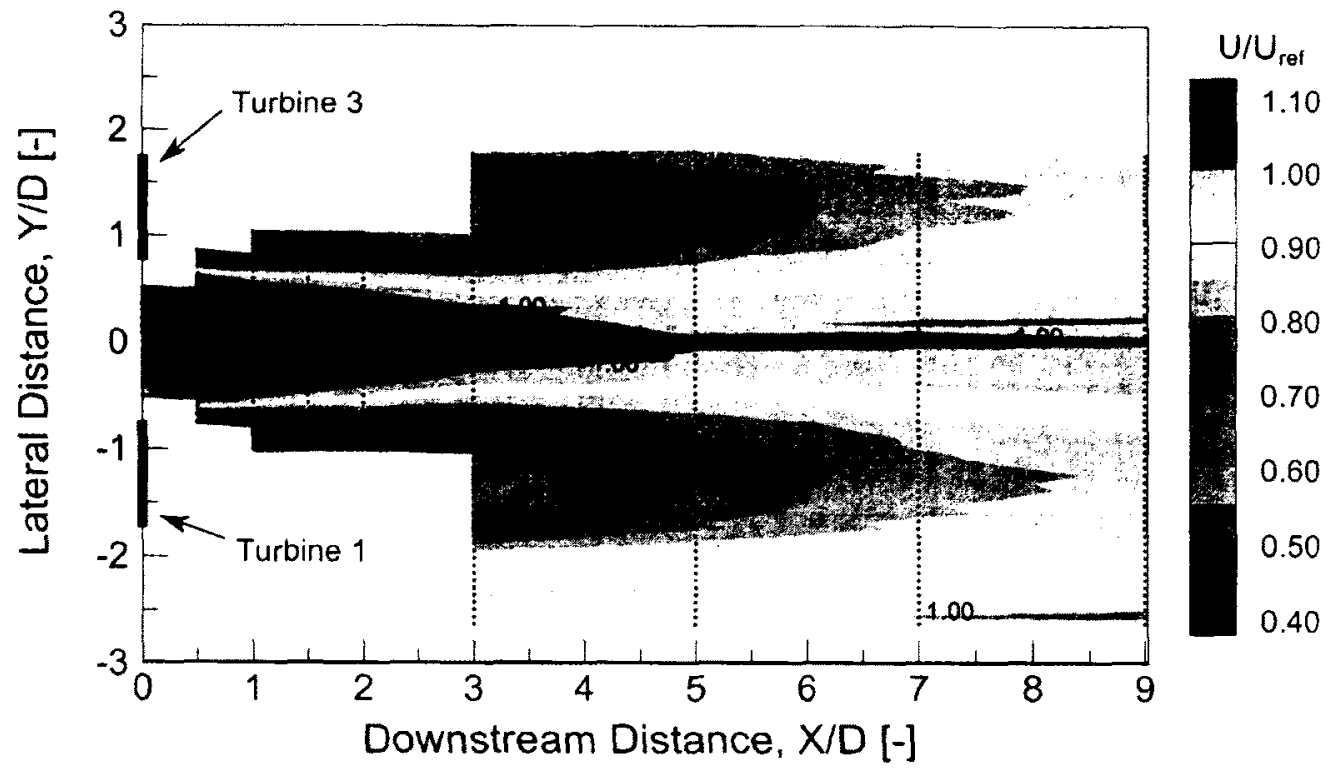

(c) Normalised velocity contours at hub height - 1.5D gap.

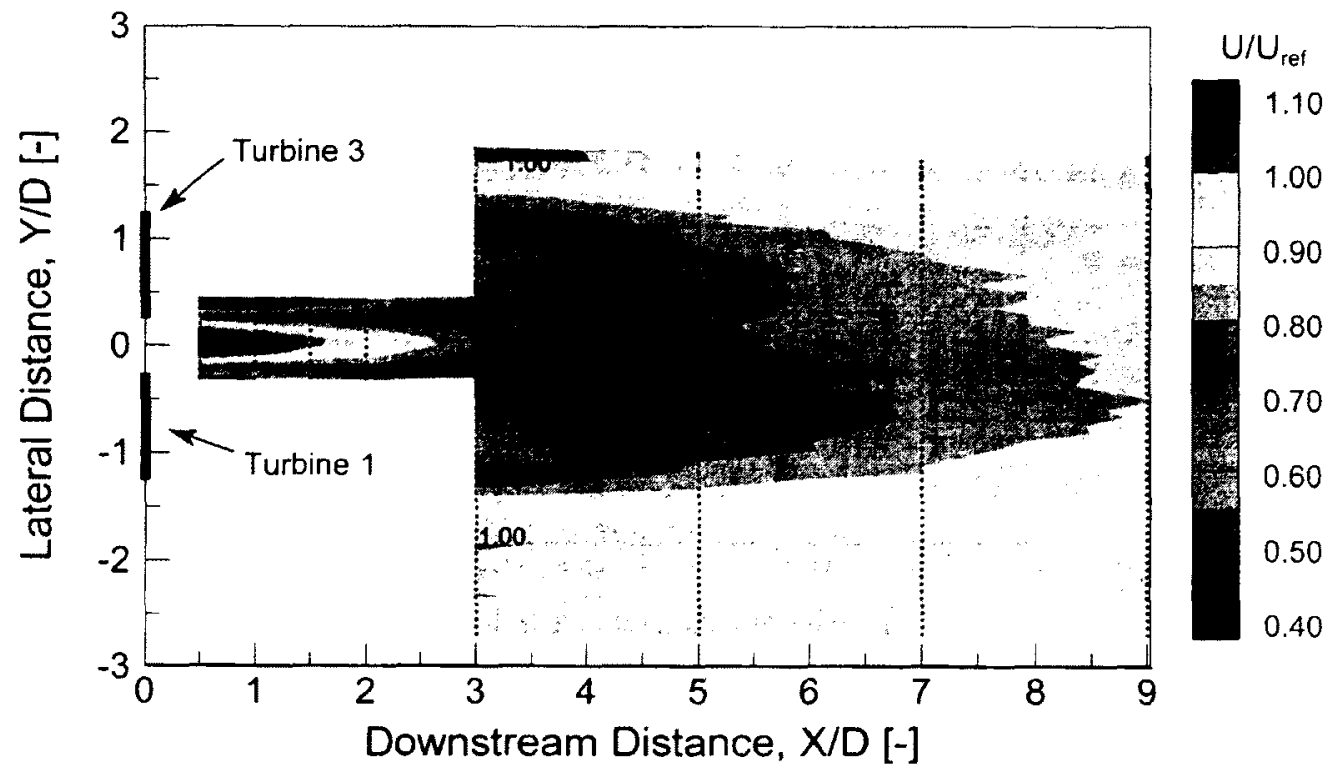

(d) Normalised velocity contours at hub height $-0.5 \mathrm{D}$ gap.

Figure 6.16 (continued). 
The flow acceleration observed in the gaps between the narrowly-spaced rotors in Figure 6.16 demonstrates what is being identified in this thesis as an in-field blockage effect. This effect is caused by the relative proximity of the rotors and their wakes and results in a constriction of the flow between adjacent rotors. The constriction created by the in-field blockage between neighbouring rotors will result in a core of higher speed through the gap that extends more than $2.5 \mathrm{D}$ downstream. This behaviour is also observed to be associated with a narrowed wake in the region encompassing the gap.

The in-field blockage effect was investigated further by plotting the centreline speed as a function of downstream distance for the four gap widths that were evaluated. The normalised centreline speed in the near wake region is plotted in Figure 6.17(a) for gap widths ranging from $3 \mathrm{D}$ to $1.5 \mathrm{D}$. The curves highlight the region of increased speed that is found within $3 \mathrm{D}$ of the upstream rotor plane, which is a maximum between $0.5 \mathrm{D}$ and 1D. The centreline speed is not representative of the more general flowfield in the gap region, however, and a more representative measure of the power generation capability of the wind would be the flow rate through the vertical planes in the gap. The data required to determine the planar speed in the gap at several different heights could not be obtained due to time constraints in the wind tunnel. The centreline speed further downstream is presented for the four gap widths in Figure 6.17(b). The 1.5D, 2D. and 3D gap width speed curves indicate that the two wakes do not merge together. The $0.5 \mathrm{D}$ gap width leads to a significant increase in speed from $0.5 \mathrm{D}$ to $1 \mathrm{D}$. followed by a decrease to $U / U_{\text {ref }} \approx 0.8$ as the adjacent wakes merge quickly in this case. The centreline speed with $G=0.5 D$ gradually recovers downstream.

It was also observed in the contour plots in Figure 6.16 that the portion of the wake adjacent to the gap appeared to narrow. The wake outline in each of the studied cases was evaluated in a similar manner to the wake profile determined earlier from the single rotor. The wake outlines at the three narrowest gap widths are compared in Figure 6.18 


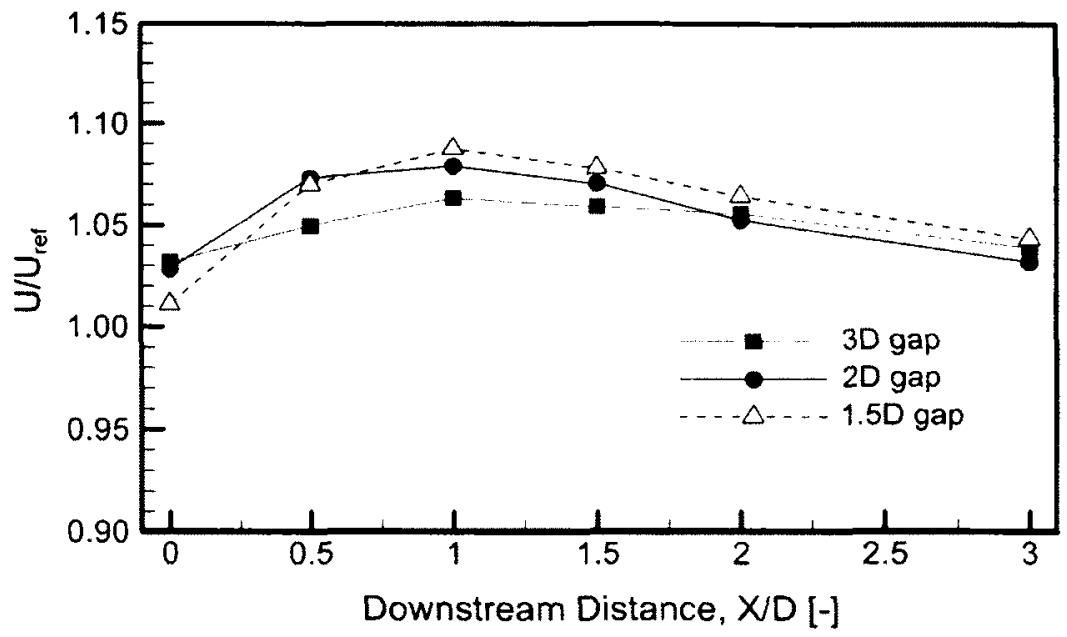

(a) Centreline speed in the near wake

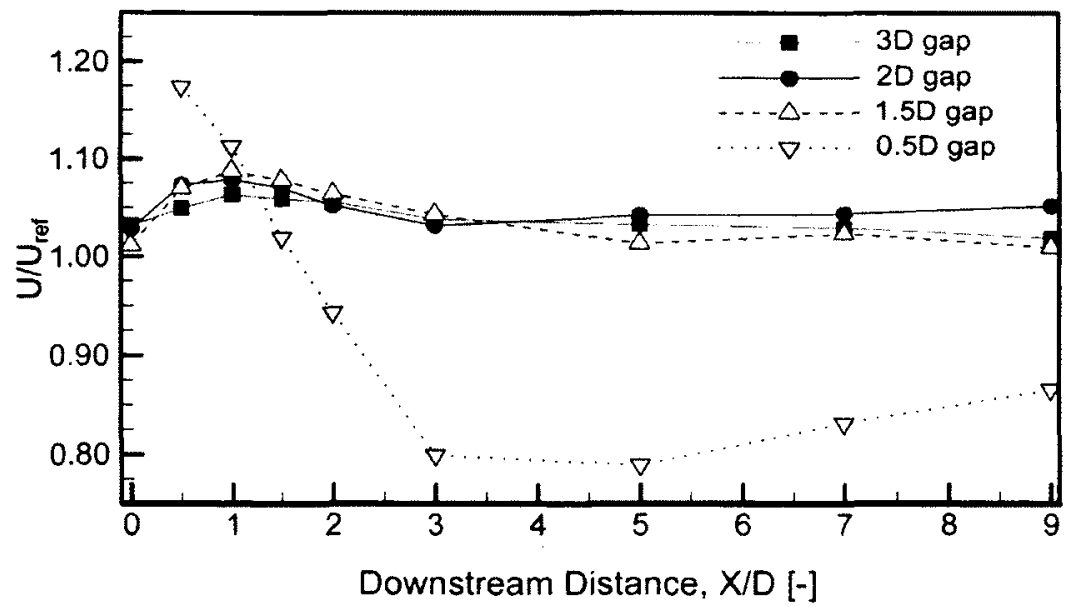

(b) Centreline speed in the far wake.

Figure 6.17: Normalised centreline speed at several gap widths.

and illustrate the narrowed wake that is associated with the flow acceleration in the gap.

\subsection{Power augmentation in three-rotor configurations}

The increased speed resulting from the close proximity of the turbines creates a unique opportunity to increase the power output of wind farms by placing an additional turbine in this region. In order to capitalise on the flow acceleration created by the in-field blockage 


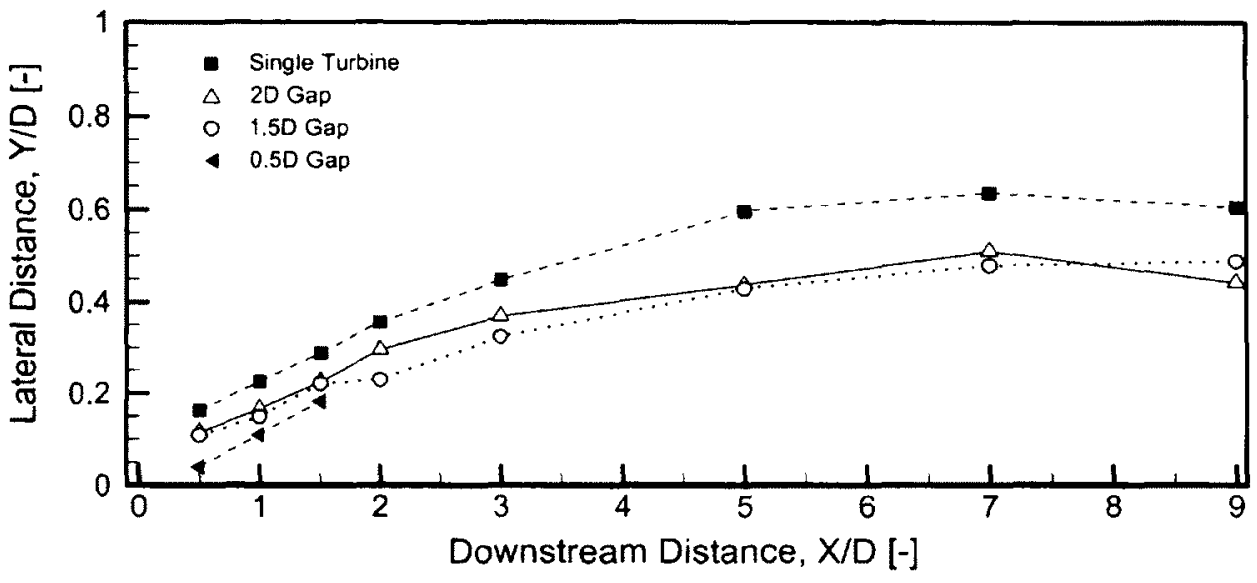

Figure 6.18: Wake profile as a function of the gap width.

effect, a multi-rotor study was conducted using three wind turbines. The wind turbines were arranged as shown in Figure 6.19, where the outer upstream wind turbines were separated by a gap distance $(G)$ and adjacent rotors had a tip clearance distance of $S_{\text {tip }}$. The longitudinal setback of the central turbine, Turbine 2, was then varied for a fixed outer turbine configuration, as indicated in Figure 6.19(b). Nine downstream setback locations were considered for each upstream gap width and the power output was recorded in each instance.

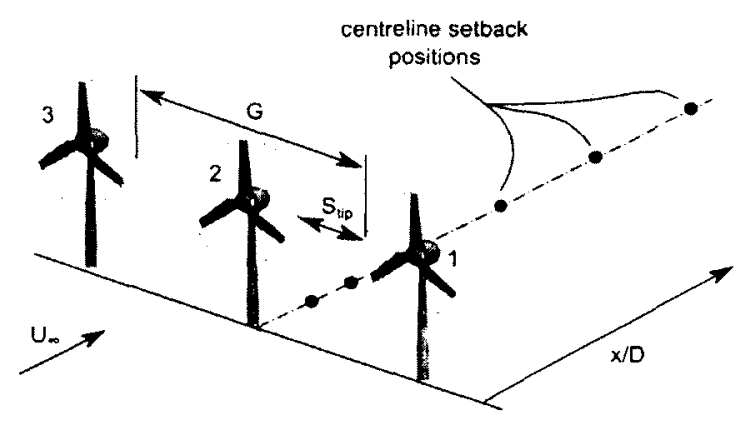

(a) Three laterally-aligned turbines.

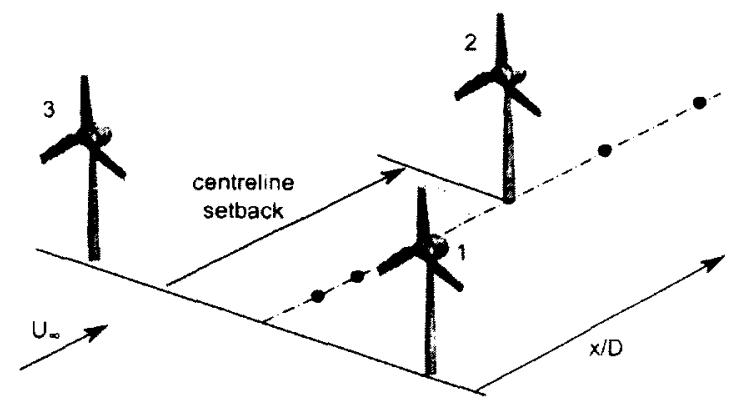

(b) Centre turbine with a downstream setback.

Figure 6.19: Schematic of the gap in three-rotor configurations.

The effect of adding a third turbine in the region of increased speed is illustrated in Figure 6.20(a). The three laterally-aligned turbines had an outer gap width of 3D. corresponding to $S_{t i p}=1 \mathrm{D}$. The speed contours highlight distinct regions of higher speed 
wind passing between the adjacent rotors. The contours also seem to indicate that the wake from the middle turbine recovers more rapidly than the outer wake. This is attributed to the high speed plumes on either side of the rotor. which create a larger shear with the edge of the inner wake and promote more rapid momentum transfer and wake recovery. The effect of adding the third. central rotor to this configuration is highlighted in Figure $6.20(\mathrm{~b})$. The power results were corrected for the speed variation in the wind tunnel and represent the increase (or decrease) in power generated by a particular turbine at that location. A modest increase in power output of $2.5 \%$ to $3 \%$ is observed by Turbines 1 and 2 in this case.

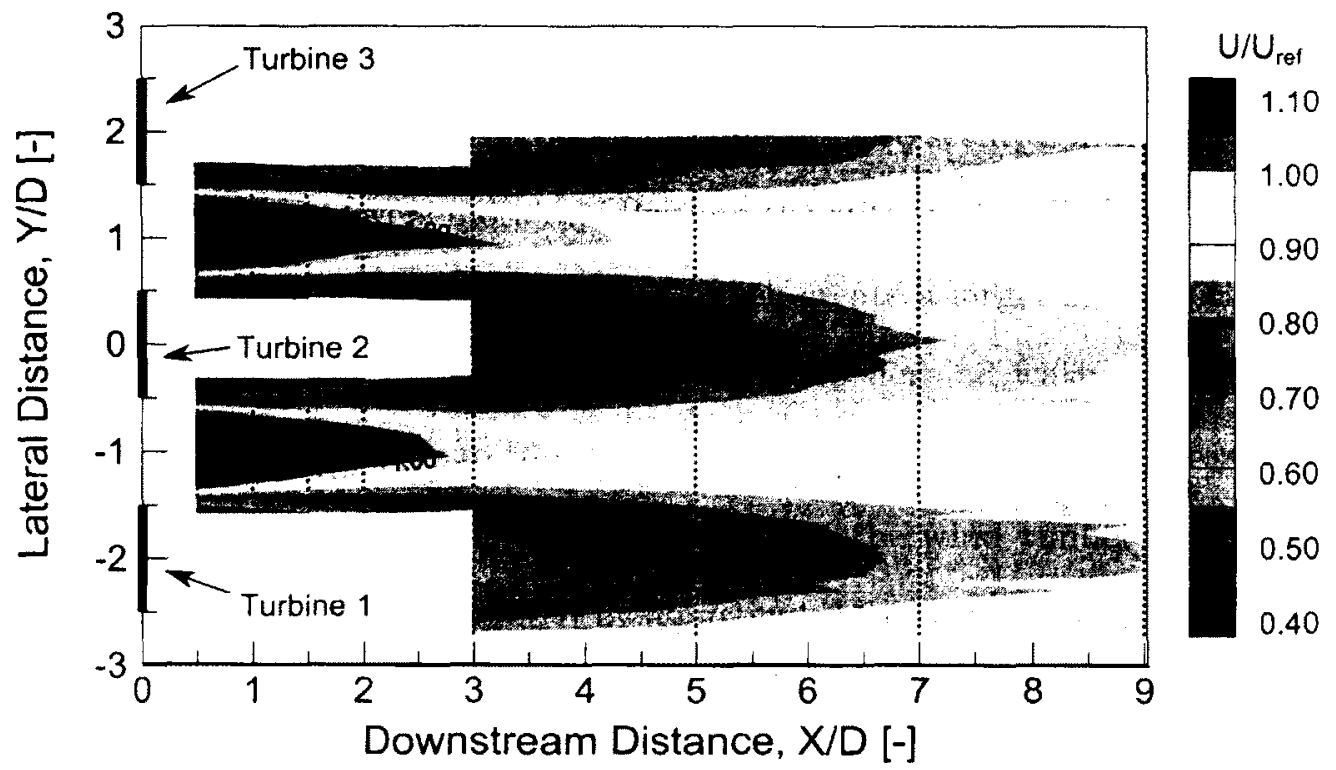

(a) Normalised speed contours at hub height.

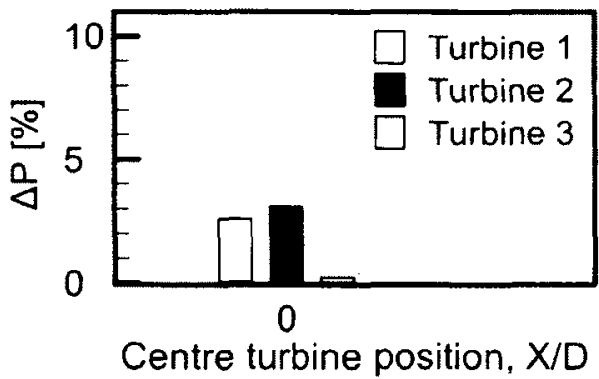

(b) Change in power output.

Figure 6.20: Speed contours and relative power output $-G=3 \mathrm{D} . S_{\text {tip }}=1 \mathrm{D}$. Three laterally-aligned turbines. 
The effect of a narrower gap with three laterally-aligned turbines is presented in Figure 6.21 (a). where $G=2 \mathrm{D}$ and $S_{t i p}$ is reduced to $0.5 \mathrm{D}$. The speed contours highlight a broad wake behind the three rotors and although the central rotor's wake appears to recover slightly earlier than the outer turbine wakes. the wake recovery distance is longer than that observed with a wider gap in Figure 6.20. The narrowed gap leads to the beneficial power increase observed in Figure 6.21(b). The power output of all three rotors is increased relative to their respective isolated power outputs and Turbines 2 and 3 each exhibit an increase of approximately $7 \%$.

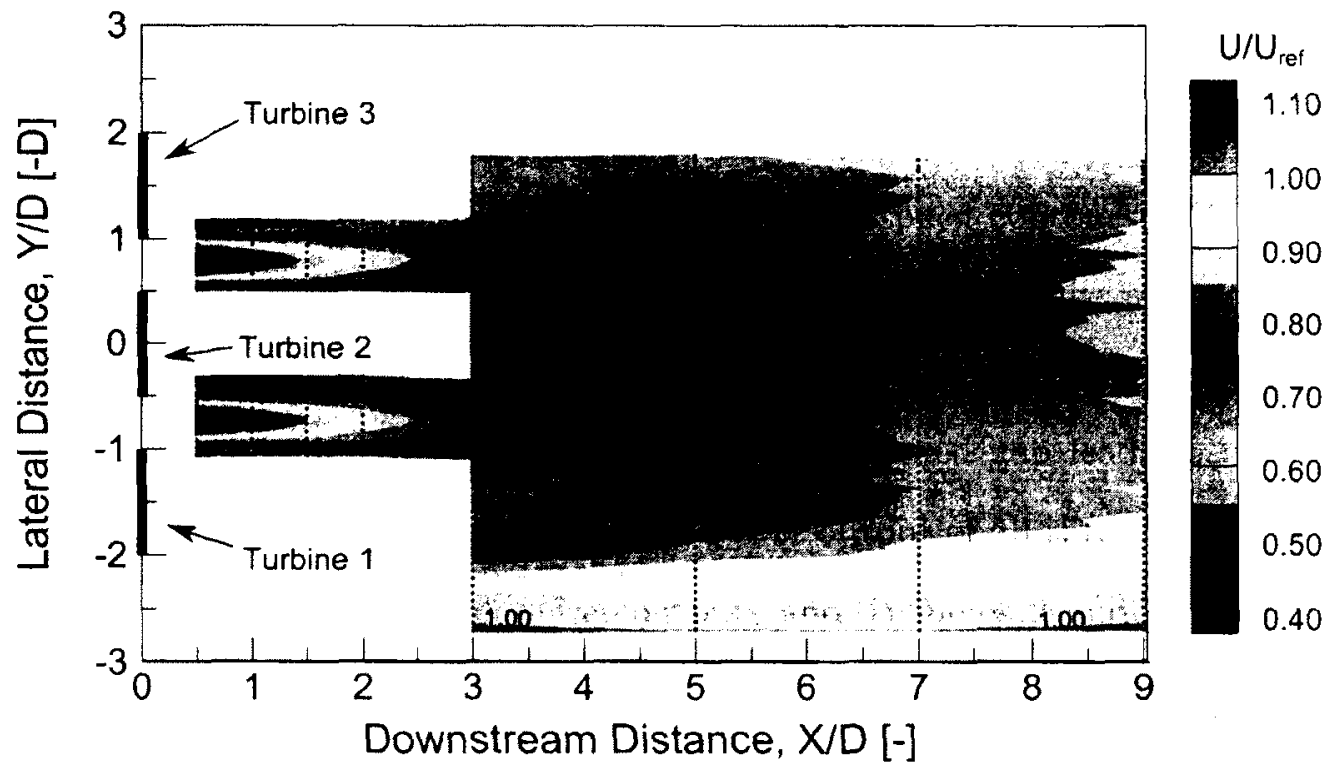

(a) Normalised speed contours at hub height.

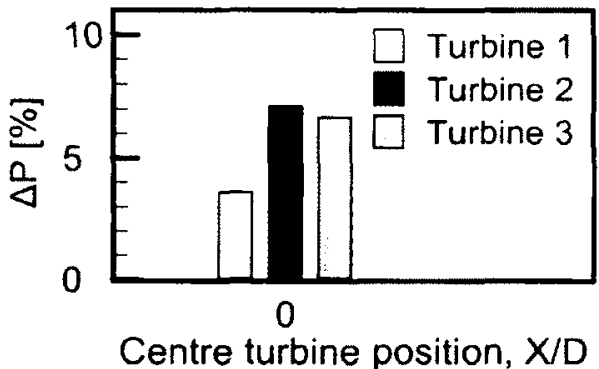

(b) Change in power output.

Figure 6.21: Velocity contours and relative power output $-G=2 \mathrm{D}, S_{\text {tip }}=0.5 \mathrm{D}$ Three laterally-aligned turbines. 
The configuration with $G=2 \mathrm{D}$ and three laterally-aligned turbines was also used to assess the repeatability of the experimental measurements. Hub height speed traverses were conducted downstream of the three-rotor configuration on October 1, 2012 and again as part of a separate series of measurements conducted between October 12-22. 2012. The normalised velocity profiles in the wakes of the three upstream turbines at downstream planes ranging from $1 \mathrm{D}$ to $9 \mathrm{D}$ are compared in Figure 6.22. Although some disagreement between the wake profiles can be observed near $Y / D=2$ at the $3 \mathrm{D}$ plane, there was generally good agreement between the two sets of results.
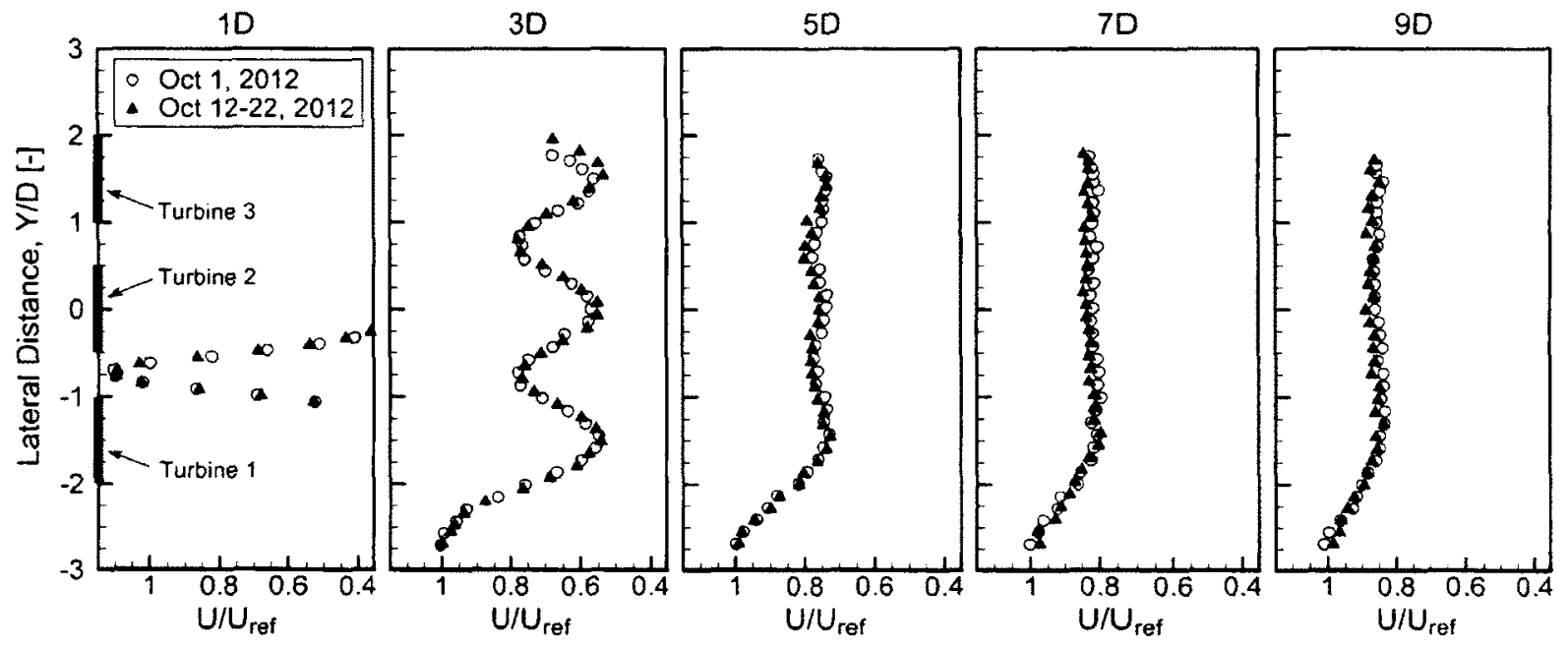

Figure 6.22: Repeatability of speed measurements.

Three laterally-aligned turbines $-G=2 \mathrm{D} . S_{\text {tip }}=0.5 \mathrm{D}$.

The centreline speed curve that was previously-presented in Figure 6.17(a) indicated that the maximum speed increase with $G=2 \mathrm{D}$ occurred at $X / \mathrm{D}=1$. The effect of a centreline setback of $1 \mathrm{D}$ for the middle rotor on the flowfield was therefore evaluated and can be seen in Figure 6.23. The speed contour plot in Figure 6.23(a) demonstrates that the wakes from the outer turbines are deformed and that a region of higher speed is present near the centre turbine. A broad, partially-merged wake is present downstream. but the downstream speed appears to have recovered more rapidly than when the three rotors were longitudinally-aligned. The power output in the case of a $2 \mathrm{D}$ gap and a $1 \mathrm{D}$ centreline 
setback is presented in Figure 6.23(b) and highlights that Turbine 2 generates $10 \%$ more power than the same turbine in isolation.

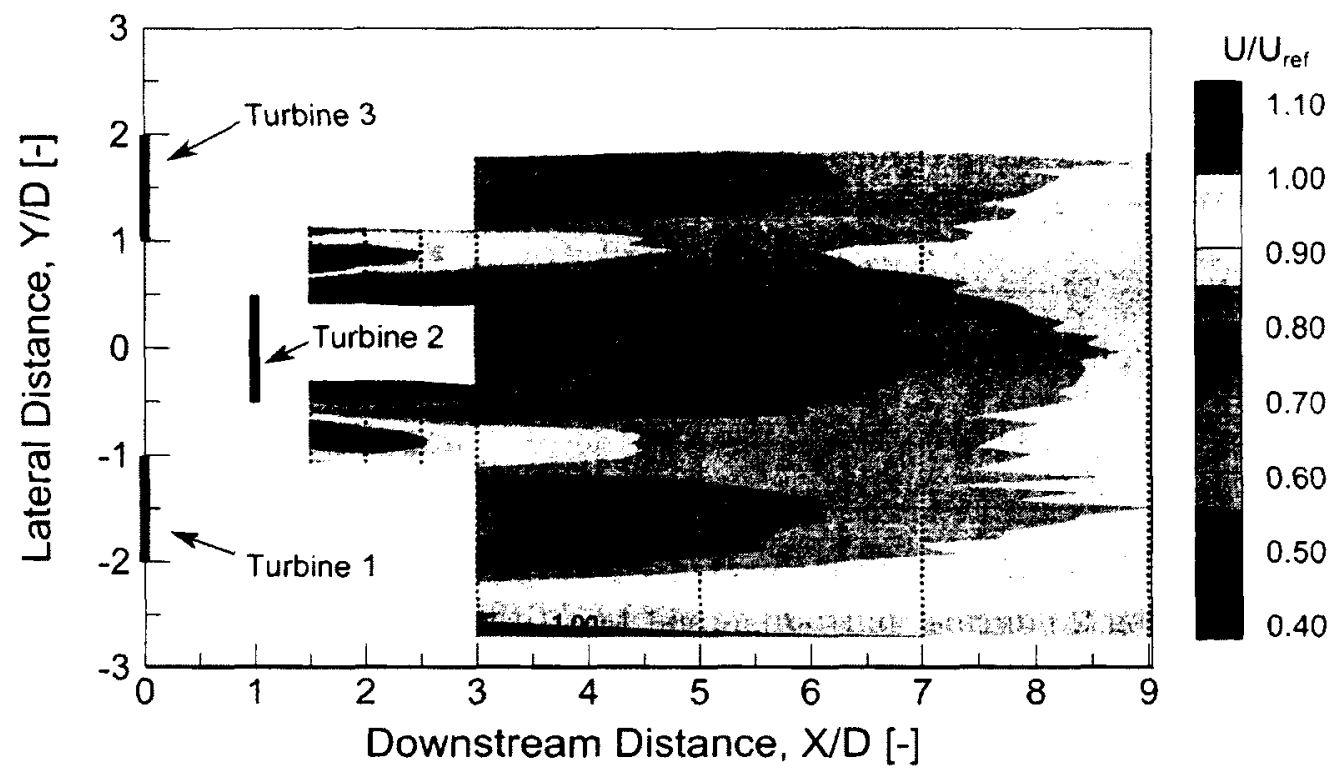

(a) Normalised speed contours at hub height.

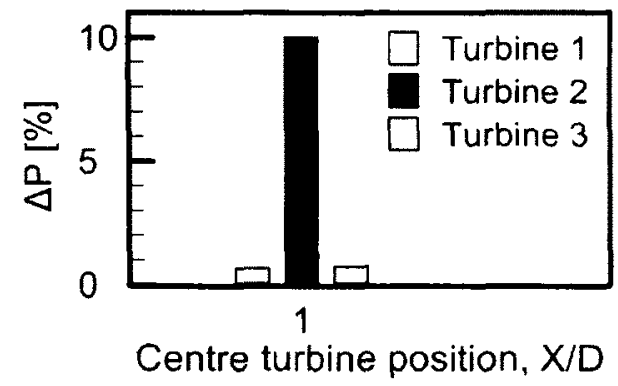

(b) Change in power output.

Figure 6.23: Velocity contours and relative power output $-G=2 \mathrm{D}, S_{t i p}=0.5 \mathrm{D}$. Centre turbine has a $1 \mathrm{D}$ setback.

\subsubsection{Performance in a rough boundary layer}

The flowfields described above indicated that the power output of lateral wind farm configurations could be increased by placing a turbine in the region of increased speed caused by the in-field blockage effect. The potential gains in power output from the remaining configurations (three gap widths and nine longitudinal setback distances per gap width) 
were evaluated using three wind turbines in a rough boundary layer. As before, the power output from each turbine was position-corrected in order to account for the speed variation in the test section.

Figure 6.24(a) plots the change in power generated by each rotor as a function of the longitudinal setback of the centre rotor when the gap width was $3 \mathrm{D}$, corresponding to a tipto-tip separation of $1 \mathrm{D}$. The centre rotor, Turbine 2 , exhibits an increase in power output when located $1 \mathrm{D}$ to $2.5 \mathrm{D}$ downstream of the upstream rotors and exhibits a maximum increase in power output of $9 \%$ with a $1 \mathrm{D}$ longitudinal setback and a $3 \mathrm{D}$ gap.

The gap was narrowed to $2 \mathrm{D}$, corresponding to $S_{t i p}=0.5 \mathrm{D}$, and the resulting change in the power output of the three rotors is presented in Figure 6.24(b). A significant increase in the power output of Turbine 2 is observed in Figure 6.24(b) as its position is varied downstream. Turbine 2 experiences a maximum increase in power output of $10.5 \%$ with a $1.5 \mathrm{D}$ longitudinal setback, but this benefit is reduced as the rotor is moved further downstream. Turbines 1 and 3 also exhibit a notable increase in power output when the setback of the centre turbine is minimized.

Similar characteristics can be noted when the gap was $1.5 \mathrm{D}$. corresponding to $S_{t i p}=$ $0.25 \mathrm{D}$, in Figure 6.24 (c). Turbine 2 produces a maximum power increase of $7 \%$ with a 1D longitudinal setback, but exhibits a smaller overall increase in power compared to the previous case. The outer turbines, however, generate a moderate increase in power output when all three rotors are operating in the same lateral plane.

The trends observed in the power output of the middle turbine at the three gap widths qualitatively follows the centreline speed curve that was previously-presented in Figure 6.17(a). The average reference speed in the test section for the three-rotor tests had a variation of $8.03 \pm 0.03 \mathrm{~m} / \mathrm{s}$, which represents a speed variation of $0.37 \%$ from the mean speed. The mean speed in the three-rotor tests was less than $1 \%$ higher than the mean speed 


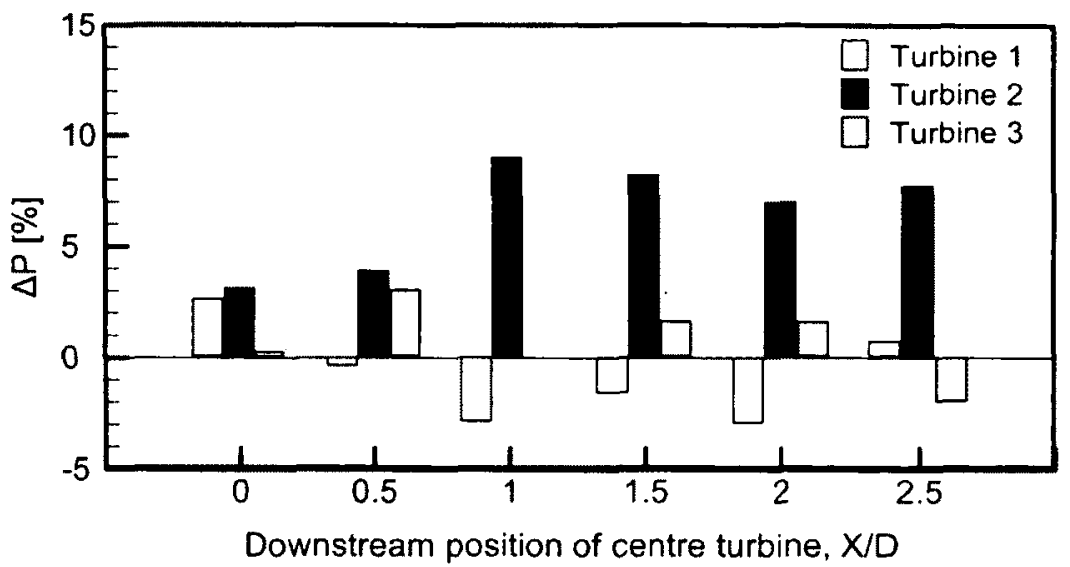

(a) Gap width of 3D, $S_{t z p}=1 \mathrm{D}$

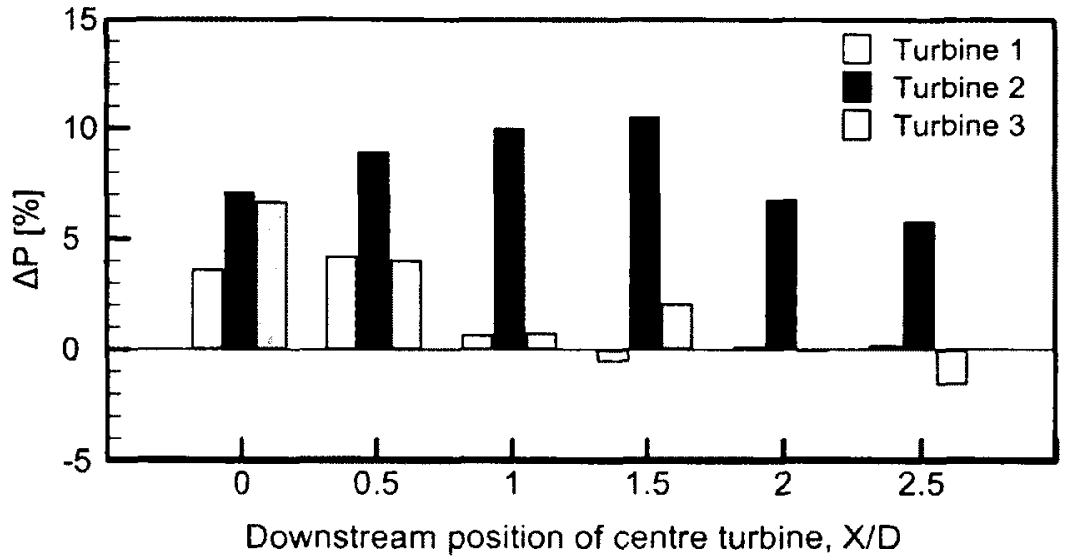

(b) Gap width of $2 \mathrm{D}, S_{t \imath p}=0.5 \mathrm{D}$.

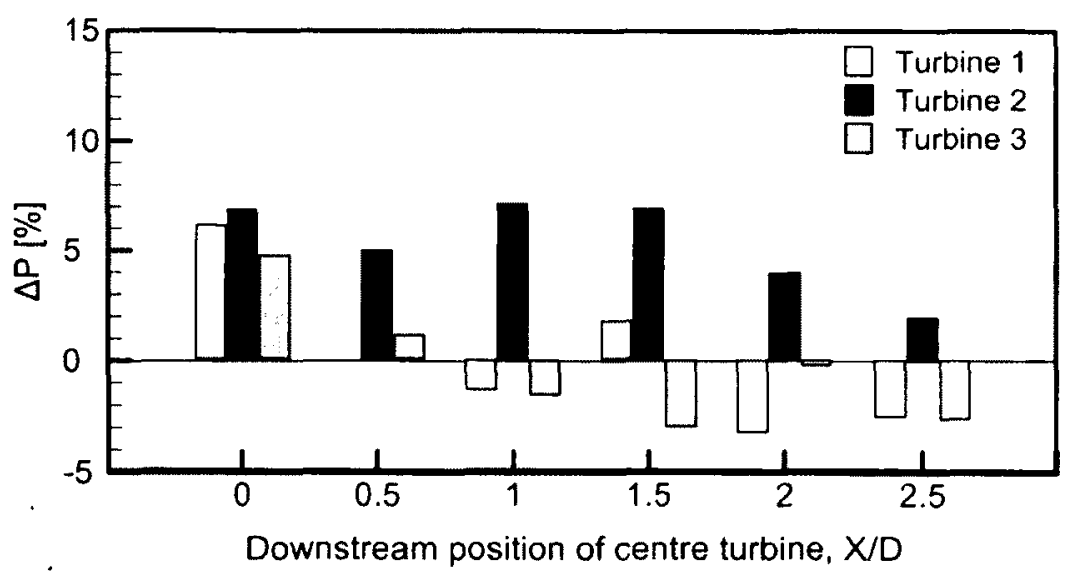

(c) Gap width of $1.5 \mathrm{D}, S_{t z p}=0.25 \mathrm{D}$.

Figure 6.24: Closely-spaced wind farm performance in a rough boundary layer. 
in the single-rotor studies. This variation was estimated to result in a maximum variation in the magnitude of the recorded power of $\Delta P=2.5 \%$. By comparison. the uncertainty due to the measurement of voltage and resistance in the power calculation was approximately $\Delta P=0.125 \%$. Some additional variations were noted as an asymmetry in the power output between Turbines 1 and 3 . The difference between the power output of these two rotors generally falls within the $\pm 2.5 \%$ bounds noted for the uncertainty in the power output of the turbines. Additionally, in cases where three rotors are aligned in the same lateral plane. some of the asymmetry may be due to a Magnus effect caused by the fact that all three turbines are rotating in the same direction.

The performance results obtained in the various wind turbine configurations have identified that the power output of a centre turbine may be increased due to the narrow gap created by two upstream rotors. Although efforts were taken to minimise the effect of blockage on the power results, it must be recognised that the tests were conducted in a confined environment. The overall blockage of the three rotors was less than $5 \%$. but the combined diameters of the three rotors represented $35 \%$ of the wind tunnel width and this may have had an effect on the outcome of the study that could not be quantified in the experiment. The author is confident that the gain in power is not purely due to the level of overall blockage in the tunnel, however. A similar in-field blockage effect occurred in the static mesh disc experiment conducted by Myers and Bahaj in a water flume which had lower levels of blockage than those observed in the current research [46]. In the work of Myers and Bahaj, three $10 \mathrm{~cm}$ diameter static discs were configured in a $1.35 \mathrm{~m}$ wide channel and the frontal area of the three discs created an overall blockage of $1.1 \%$. while the cumulative diameters of the discs accounted for $22 \%$ of the width of the channel [46]. Additionally, there was 5D separation between the outer disc edge and the walls of the channel [46]. The authors observed an increase in the flow speed between two outer discs of approximately $4-5 \%$ at a distance $3 \mathrm{D}$ downstream [46], similar to the magnitude of the 
speed increase observed in the current work. In addition to the results obtained by Myers and Bahaj. it is expected that the overall level of blockage on the results will be minimal based on the power results observed above in Figure 6.24. When Turbine 2 is located more than $1 D$ downstream of the outer rotors. the $\Delta P$ of Turbines 1 and 3 is close to 0 and within the $\pm 2.5 \%$ error bounds estimated for $\Delta P$. This indicates that Turbines 1 and 3 are not experiencing a significant artificial blockage-induced power increase.

These results, however, represent data recorded in a low Reynolds number environment and it remains to be determined if the observed gains can be transferred to a commercialscale. Computational studies should be conducted to this effect in order to ascertain the potential performance benefits of such configurations in unbounded atmospheric flows at Reynolds numbers representative of commercial wind turbines.

\subsubsection{Performance in a smooth boundary layer}

The behaviour of closely-spaced configurations in a smooth boundary layer designed to mimic offshore conditions was subsequently evaluated with a gap width of $2 \mathrm{D}$. corresponding to $S_{t i p}=0.5 \mathrm{D}$. The results shown in Figure 6.25 exhibit similar trends as the wind farm behaviour in a rough boundary layer. The centre turbine exhibits a modest power increase when located within $2 \mathrm{D}$ of the upstream rotors. Similar to the rough boundary layer case, the outer turbines see an additional benefit when the three rotors are aligned in the same lateral plane. The increase in power observed in the smooth boundary layer case is. however, lower than that observed with a rough boundary layer. This is partly attributed to the observation that the velocity gradient in the rough $A B L$ test results in a higher speed over the upper half of the rotor disc than in the smooth case and these results may be unique to this particular experiment. 


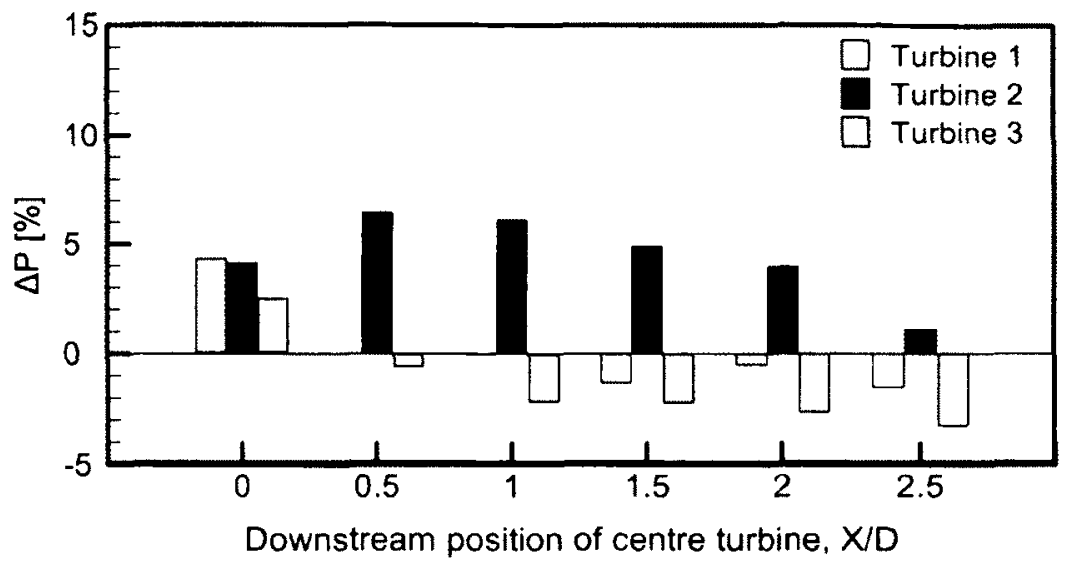

Figure 6.25: Closely-spaced wind farm performance in a smooth boundary layer $-G=2 \mathrm{D}$.

$$
S_{t i p}=0.5 \mathrm{D} \text {. }
$$

\subsubsection{Downstream effects}

The effect of an upstream gap on the power output of a rotor located 6.5D downstream was subsequently evaluated in a rough boundary layer. This was done in order to gain an understanding of the wake merging behaviour of the upstream wakes on the power generated by a downstream turbine. The downstream distance at which the upstream wakes merge will be a function of the lateral gap between the two upstream rotors and this behaviour was observed earlier in Figure 6.16. Figure 6.26 demonstrates that the reduced speed from the merging upstream wakes results in a large decrease in the power output of the longitudinally-setback rotor when the gap is less than $1.5 \mathrm{D}$. The wakes are partiallymerged $6.5 \mathrm{D}$ downstream when the upstream gap is $1 \mathrm{D}$. resulting in a decrease in power of approximately $20 \%$ for Turbine 2 . The downstream rotor's power output is reduced by $42 \%$ when the gap between the upstream rotors is further reduced to $0.5 \mathrm{D}$.

The magnitude of the losses experienced by the downstream rotor with two upstream. laterally-spaced rotors is compared to the typical case where two rotors are aligned in a purely longitudinal manner. This corresponds to a gap width of 0 in Figure 6.26 and it can be observed that the downstream turbine experiences a reduction in power that is similar 
in magnitude to a three-rotor configuration with a gap width of $0.5 \mathrm{D}$.

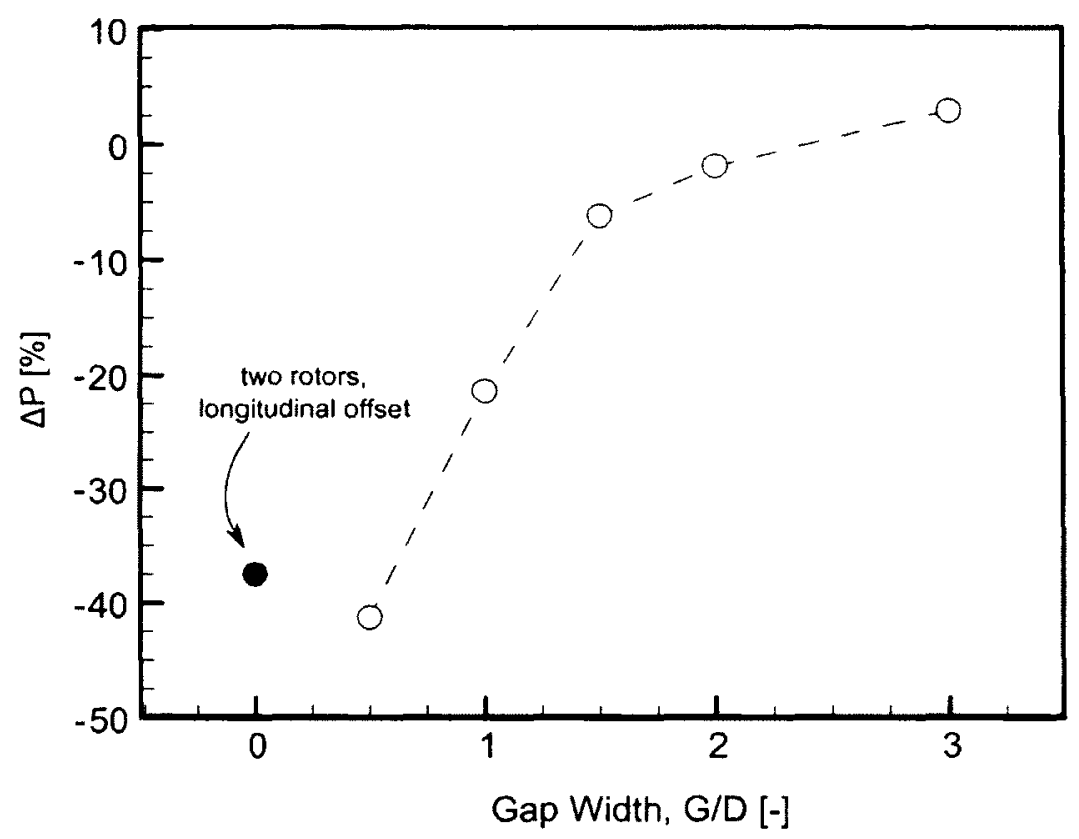

Figure 6.26: Effect of the gap on the output of a rotor located 6.5D downstream.

\subsubsection{Off-design conditions}

Several of the above-mentioned wind farm configurations resulted in an increase in power output of greater than $10 \%$. These tests were conducted in axial flow conditions. however, and represent the ideal situation where the wind is perpendicular to the turbine configuration. One of the configurations was evaluated with small changes in the wind angle $(\gamma)$ of $5^{\circ}$ and $10^{\circ}$ in order to evaluate the sensitivity of the in-field blockage effect to small changes in the wind direction. In order to conduct the directional configuration tests. the floor of the wind tunnel was replaced with the original floor containing a turntable. A change in the wind direction was mimicked in the wind tunnel by rotating the entire wind farm configuration on the turntable. Once the turntable was rotated to the desired angle. the turbines were rotated into the wind in order to mimic turbine behaviour in a wind farm operating with a change in wind direction. The turntable arrangement is represented 
schematically in Figure 6.27 .

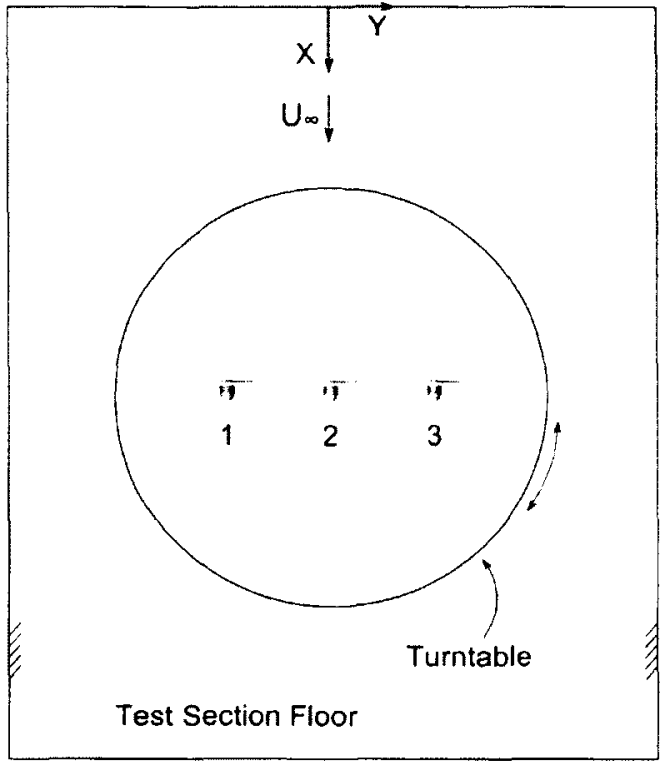

(a) Turbines with zero yaw.

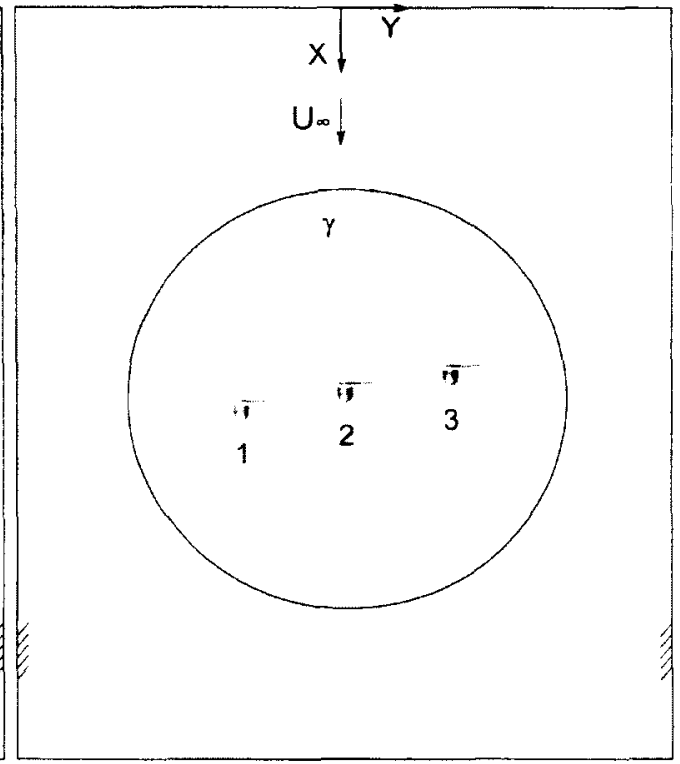

(b) Turbines with a $10^{\circ}$ yaw angle

Figure 6.27: Schematic of three turbines with $S_{t i p}=0.5 \mathrm{D}$ in yawed configurations.

Prior to conducting the tests with three wind turbines, the single turbine position correction procedure was followed in order to account for variations in the wind speed within the test section. Turbines 1,2 , and 3 were each tested in isolation in their relative position in the $0^{\circ}$ wind direction configuration. As before. the power output of the turbines in the wind farm configuration was assessed as the change in power of a turbine at a particular location from its output in isolation. The directional performance of a configuration consisting of three laterally-aligned turbines with a gap of $2 \mathrm{D}$ and $S_{\text {tip }}=0.5 \mathrm{D}$ was evaluated at $0^{\circ} .5^{\circ}$. $10^{\circ}$, and $-10^{\circ}$. The change in power for each of the three rotors is plotted as a function of the wind angle in Figure 6.28. The change in power of the three turbines at an angle of $0^{\circ}$ compares well with the previous results presented in Figure 6.24(b), although the results presented here have a $\Delta P$ that is $1-2 \%$ lower than the previous results. In general. at these small wind angles, the power output of Turbine 2 increases as the wind angle changes from $0^{\circ}$ to $10^{\circ}$. Additionally, there is good agreement between the performance of the wind farm 
at $-10^{\circ}$ and $10^{\circ}$. These results highlight that the closely-spaced wind farm configurations proposed in this thesis may be particularly well suited to sites where the wind has a narrow distribution of wind directions.

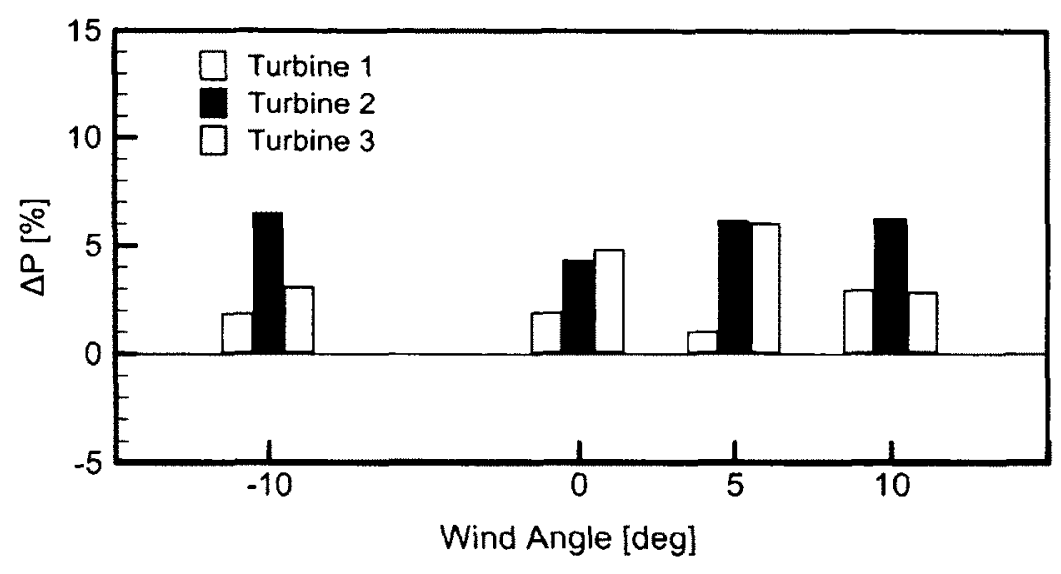

Figure 6.28: Power output of three laterally-aligned turbines with $S_{\text {tip }}=0.5 \mathrm{D}$ at various wind angles.

Although only small changes in the wind angle were evaluated, it is expected that the power output of Turbine 2 will decrease as the wind angle increases from $20^{\circ}$ to $30^{\circ}$. Turbine 2 will begin to be partially-blanketed by the wake from Turbine 3 in this range of angles. based on the relative position of the turbines and the expected wake expansion behaviour.

The scenario with a $90^{\circ}$ wind angle would result in turbines aligned longitudinally with only $2 \mathrm{D}$ separation between the rotors. This situation would result in increased unsteady loading and a drop in the power output from the downstream rotors on the order of $50 \%$. This situation, along with other wind angles that result in partially-waked conditions for the downstream rotors could be mitigated in practice using the concept of sector management. This technique is frequently used by wind farm operators to shut down certain turbines when the wind is coming from a particular direction, or range of directions, in order to mitigate terrain effects or wake effects that could create undesirable loading conditions on the rotor. 


\subsubsection{Cumulative power increase}

The cumulative effect of the rotor layouts was assessed by adding the change in power $\left(\Sigma \Delta P=\Delta P_{1}+\Delta P_{2}+\Delta P_{3}\right)$ for Turbines 1, 2. and 3 in all of the rotor configurations. This allowed the wind farm system to be evaluated compared to the same three rotors in isolation. The cumulative power increase as a function of the centreline setback of the middle turbine can be observed in Figure 6.29 for three gap widths in the rough boundary layer. The maximum cumulative increase in power was observed to be $17.8 \%$, and occurred when the rotors did not have a longitudinal offset. Turbines 1 and 3 see an added benefit when all three rotors are aligned laterally, and this results in the highest net benefit. despite the fact that Rotor 2 sees its maximum benefit when located further downstream. The downstream range in which a significant benefit is observed appears to narrow with a smaller gap width. A gap width of $2 \mathrm{D}$, corresponding to tip separation of $0.5 \mathrm{D}$. produces the best range of large power increases $(>10 \%)$ and this occurs when the centreline setback is within $1.5 \mathrm{D}$

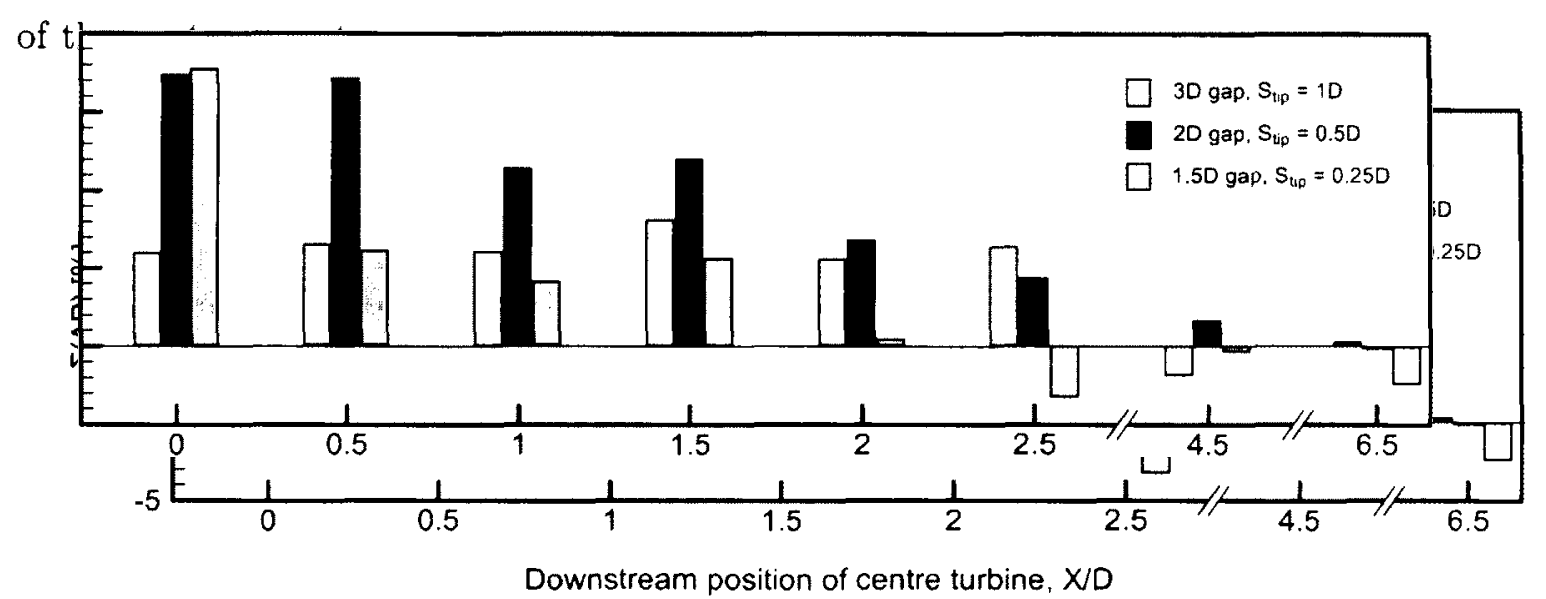

Figure 6.29: Cumulative change in the power output as a function of the gap width in a rough boundary layer.

The cumulative change in power was also evaluated as a function of the boundary layer profile when the gap width was $2 \mathrm{D}$ and can be seen in Figure 6.30. A greater benefit is observed to occur with a rough boundary layer profile than with the smooth profile and 
the maximum benefit in both cases occurs when the three turbines are aligned in the same lateral plane.

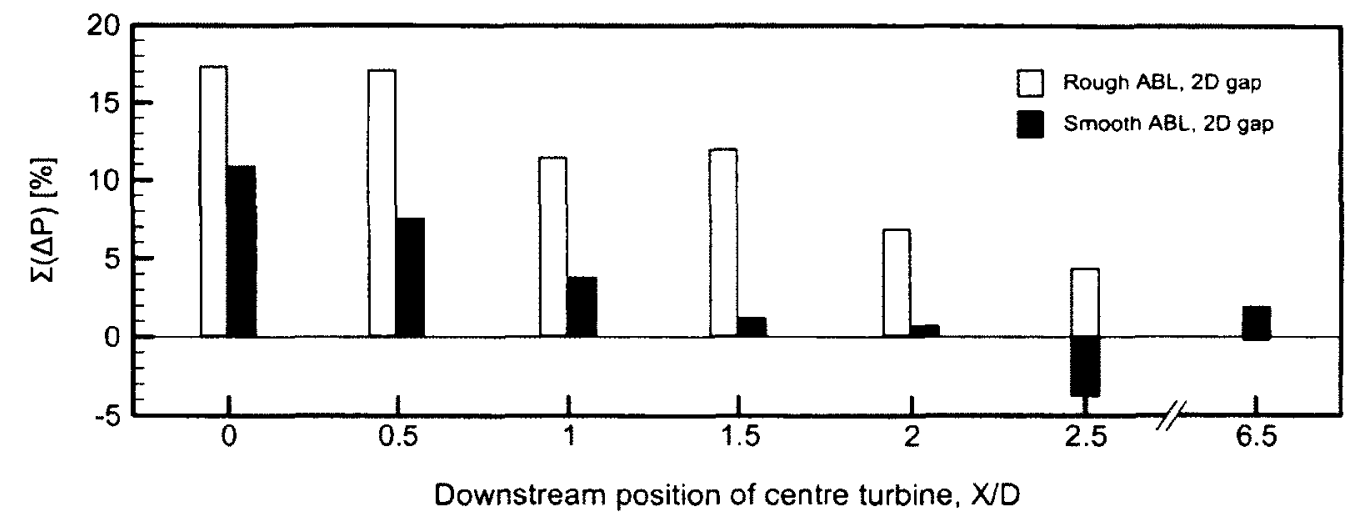

Figure 6.30: Cumulative change in the power output as a function of the boundary layer.

The configurations identified in this thesis not only present the opportunity to reduce losses incurred due to wake effects, but may also increase the power output of turbines in mean annual wind speeds. These results, however, are only representative of the threerotor configurations under consideration and their interpretation should take into account the fact that the experiments were conducted in a confined environment at a low Reynolds number. It is recommended that additional computational simulations be conducted in order to evaluate these configurations in unbounded atmospheric flows at Reynolds numbers representative of commercial-scale conditions.

\subsection{Summary}

The current work explored the effect of closely-spaced lateral wind farm configurations with tip-to-tip separation distances that ranged from $1 \mathrm{D}$ to $0.25 \mathrm{D}$. The in-field blockage created as a result of the narrow lateral spacing increases the wind speed in between adjacent wind turbines and may be able to increase the power output of the wind farm. This beneficial flow acceleration may have the potential to reduce onshore land use requirements 
in addition to increasing the wind farm capacity factor at wind farm sites with particular wind distribution characteristics.

- Experiments conducted with two longitudinally-aligned wind turbines as well as with a grid-aligned $3 \times 3$ wind farm confirmed that losses due to wake effects in such configurations are typically $20 \%-40 \%$.

- Hot-wire measurements conducted between two closely-spaced laterally-aligned turbines with different gap widths identified that a synthetic in-field blockage effect occurs between the rotors that results in a region of increased wind speed. The region of increased speed extends from the upstream rotor plane to $2.5 \mathrm{D}$ downstream.

- A systematic study of the effect of scaled three-rotor configurations indicated that a rotor placed in the region of increased speed generated by two closely-spaced upstream rotors generated 5\%-11\% more power than the same rotor in isolation. It is recommended that computational simulations be conducted using grid-based CFD or a discrete vortex method in order to identify the behaviour of such wind turbine configurations in unbounded atmospheric flows at commercial-scale Reynolds numbers.

- The in-field blockage effect identified with closely-spaced lateral wind turbine configurations may offer the potential to increase the annual capacity factor of a wind farm by increasing the energy yield in mean annual wind speeds while requiring less land for a wind farm. 


\section{Chapter 7}

\section{Conclusions, Academic}

\section{Contributions, and Future Work}

The goals of the research involved characterising experimental testing limitations using scaled wind turbines and investigating the ability of closely-spaced wind turbine configurations to increase the performance of a wind farm. The research was conducted using a predominantly experimental approach and investigated the effects of blockage and Reynolds number on the development of the initial wake region of a single turbine as well as the performance and wake characteristics of multi-turbine wind farm configurations.

A scaled wind turbine was designed to support the experimental campaign. The MAAE turbine was designed to generate a thrust coefficient similar to that observed on commercialscale turbines and had a normalised chord that was scaled up relative to full-scale designs in order to increase the Reynolds number reached by the blades. An airfoil was selected that had adequate performance in the anticipated Reynolds number regime.

Blockage effects were characterised in the water channel at Carleton University with the scaled rotors using quantitative flow visualisation. Dye was injected into the water 
and the positions of the photographed tip vortex streaklines were used to identify the expansion of the near wake. Five rotors with different diameters were evaluated in this way. with blockage ranging from $6 \%$ to $25 \%$. The work identified that blockage greater than $10 \%$ constricted the expansion of the wake downstream of the turbine and delayed vortex pairing. The applicability of blockage corrections was verified as part of the work and this confirmed that although the recorded thrust coefficients increased with increasing blockage. the corrected coefficients collapsed to a similar magnitude.

Following the blockage experiment, the effect of the Reynolds number on the wake expansion and the thrust coefficient was evaluated using the MAAE turbine. The wake expansion was identified using the same quantitative flow visualisation technique applied in the blockage experiments. A limit above-which the wake became less sensitive to Reynolds number was identified for this particular turbine. The benefits of developing a rotor tailored for a low Reynolds number regime was contrasted with the behaviour of a geometricallyscaled rotor operated outside of its intended flow regime. A scaled version of an existing turbine from the Delft University of Technology was manufactured and was tested in the water channel. The wake and the thrust coefficients generated by the scaled turbine in a low Reynolds number environment were significantly lower than the wake and thrust coefficients produced by the medium-scale turbine due to the poor behaviour of the rotor's airfoil at low Reynolds numbers.

Finally, the performance of scaled wind farm configurations was evaluated using wind turbines with the MAAE rotor blades in the atmospheric boundary layer wind tunnel at Carleton University. The power output generated by the scaled turbines was used to characterise their performance and a single-normal hot-wire probe was used to determine the mean speed profiles in the flowfield. It was observed that when two turbines had a tipto-tip spacing of less than 3D, the speed of the flow between the rotors was increased and this was identified in the thesis as an in-field blockage effect. The increased flow speed was 
associated with a narrowing of the wake between the closely-spaced rotors and the concept of capitalising on this in-field blockage effect using a third. offset rotor was investigated. Performance measurements were conducted for 3 gap widths between the outer two turbines and a third, centre turbine was placed at 9 different downstream positions. It was identified that the power output of the wind turbines could be increased when a central rotor was placed within $2.5 \mathrm{D}$ of the upstream rotors, corresponding to the region of increased speed. This approach to planning wind farms will help to limit wake effects while providing an increase in power output at mean annual wind speeds.

\subsection{Conclusions}

The results of the thesis were grouped into three chapters that reflected the blockage. Reynolds number, and wind farm aspects of the research.

\subsubsection{Blockage effects}

- Guidelines were developed regarding the dye visualisation setup in the water channel. It is recommended that the dye setting should be maintained at or below the 'medium' dye level used in this study. This limits the effect of the dye stream on the wake expansion and on the recorded forces. Additionally, the turbine-supporting strut should be isolated from the turbine wake using a concentric pipe.

- Quantitative dye visualisation indicated that the initial wake expansion was narrowed by the presence of the walls when the blockage of the rotor was larger than $10 \%$. The effect of blockage on the initial wake expansion was significant and resulted in an initial wake expansion that was $35 \%$ to $55 \%$ narrower when blockage was increased above $10 \%$ 
- Tip vortex pairing behaviour was dependent on the quantity of solid blockage created by the rotor and blockage of $25 \%$ was observed to delay vortex pairing.

- The experimental wake expansion results with blockage ratios of $6.3 \%$ and $9.9 \%$ were compared to computational results conducted in freestream conditions. The wake behaviour in the computation was consistent with the established wake expansion trends and predicted a similar wake expansion magnitude compared to the blockageaffected experiments.

- The thrust coefficients inferred with a six degree-of-freedom balance demonstrated that the as-measured. uncorrected thrust coefficients increased with increasing blockage, although the corrected thrust coefficients collapsed to a similar magnitude. Based on the results of the force measurements, it is recommended that blockage corrections may be beneficial even when the blockage is $5-10 \%$. The research presented a link between a narrowed wake and an increased thrust coefficient as blockage was increased.

\subsubsection{Reynolds number and scaling effects}

- The wake expansion downstream of the three-bladed rotor increased with increasing Reynolds number and became less sensitive to changes above $R e_{t i p}=20,000$. The wake expansion downstream of the scaled two-bladed rotor was approximately $30 \%$ to $50 \%$ narrower than experimental results from the corresponding medium-scale experiment conducted at the Delft University of Technology. It was also observed that the tip vortex pitch was not strongly affected by Reynolds number. and was instead a function of the tip speed ratio and the lift distribution along a blade, which is characterised in this work by the tip pitch angle setting.

- The thrust coefficient was observed to increase with increasing Reynolds number for the three-bladed MAAE rotor and approached the desired range of full-scale 
thrust coefficients. The thrust coefficient in the scaled TU Delft experiments was approximately $40 \%-60 \%$ lower than the corresponding medium-scale coefficient.

- Blockage corrections reduced the magnitude of the recorded thrust coefficients by up to $9 \%$ for the MAAE rotor and by up to $6 \%$ for the scaled TU Delft rotor. This confirms the recommendations developed in Chapter 4 that suggested blockage corrections may be beneficial even when blockage is approximately $10 \%$.

- The wake expansion and thrust coefficient of a small-scale model operating in a low Reynolds number regime may not match a scaled-up version of the same rotor unless the scaled rotor geometry or airfoil is modified. As a result, it is recommended that scaled wind turbine experiments should be conducted with rotors that have been tailored to the expected Reynolds number regime. Additionally, a minimum Reynolds number corresponding a reduced variation in the lift curve, transition, and separation behaviour should be reached in small-scale experiments.

\subsubsection{In-field blockage effects in wind farms with closely-spaced wind turbines}

- Experiments conducted with two longitudinally-aligned wind turbines as well as with a grid-aligned $3 \times 3$ wind farm confirmed that losses due to wake effects in such configurations are typically $20 \%-40 \%$.

- Hot-wire measurements conducted between two closely-spaced laterally-aligned turbines with different gap widths identified that a synthetic in-field blockage effect occurs between the rotors that results in a region of increased wind speed. The region of increased speed extends from the upstream rotor plane to $2.5 \mathrm{D}$ downstream. The increase in speed in the gap was associated with a narrowing of the edge of the wake profile adjacent to the gap. 
- A systematic study of the effect of scaled three-rotor configurations indicated that a rotor placed in the region of increased speed generated by two closely-spaced upstream rotors generated $5 \%-11 \%$ more power than the same rotor in isolation.

- The in-field blockage effect identified with closely-spaced lateral wind turbine configurations may offer the potential to increase the annual capacity factor of a wind farm by increasing the energy yield in mean annual wind speeds while requiring less land for a wind farm.

\subsection{Summary of Academic Contributions}

A significant portion of the research is either in various stages of review or has been accepted for publication in academic journals and for presentation at peer reviewed conferences. The major academic contributions resulting from the current research are highlighted below:

- A scaled wind farm was developed that consisted of 9 wind turbines arranged in a grid-like layout that has been used to understand wake effects in traditional wind farm layouts [74].

- The limitations of scaled wind turbine testing were evaluated by identifying the effect of wind turbine blockage on the development of the near wake expansion. A threshold beyond which blockage in a closed test section constricts the expansion of the wake was identified for the first time in literature $[172,173]$.

- The limitations of scaling wind turbine experiments were further assessed by comparing the wake expansion and thrust force generated by a geometrically-scaled wind turbine to a medium-scale experiment [174]. The advantages of developing a scaled 
wind turbine design for a low Reynolds number testing environment were contrasted with the challenges in using a purely geometrically-scaled rotor.

- A new approach to laying out wind farms was identified. in which wind turbines are closely-spaced in the lateral direction. The close lateral spacing creates an in-field blockage effect that results in a region of increased speed between the turbines. The resulting flow acceleration in the narrow gaps between the wind turbines resulted in a notable power increase for a scaled wind farm $[175,176]$.

- The development of the wake downstream of closely-spaced wind turbine configurations was characterised and the accelerated flow in the gap between adjacent turbines was measured [177].

\subsection{Recommendations and future work}

The wind tunnel experiments in the current work indicated that the closely-spaced wind turbine configurations may have the ability to increase the performance of wind farms in mean annual wind speeds. These experiments were conducted in a constrained flow in a wind tunnel, however, and this may have introduced a source of error in the recorded power estimates. It is recommended that computational simulations be conducted using grid-based CFD or a discrete vortex method in order to identify the behaviour of such wind turbine configurations in unconstrained atmospheric flows. These simulations could initially be conducted in unbounded freestream conditions. followed by a flow situation in which the ground is represented along with an atmospheric boundary layer. The performance of the closely-spaced configurations should also be investigated at commercial-scale Reynolds numbers in order to develop a deeper understanding of how the scaled configurations investigated in this work relate to larger-scale turbines. 
Wind farm configurations with only three turbines were evaluated in the current work. While the closely-spaced configurations demonstrated the ability to increase the power output of the turbines, it would be valuable to evaluate how the array efficiency of an entire wind farm could be improved. The efficiency of a wind farm could be evaluated by modifying wind farm layout software to incorporate the design guidelines and flow behaviour identified in the experiments. The use of wind farm software would allow the configuration of a wind farm to incorporate topographical, wind distribution. and land use characteristics into the optimization of a layout. This problem may necessitate additional wind tunnel studies at a wide variety of yaw angles in order to characterise the behaviour of closelyspaced configurations in off-design conditions. Wind farm software typically uses wake models to evaluate the power production of downstream turbines. While the concept of developing closely-spaced lateral wind farm configurations would aim to limit the number of turbines in operating in waked conditions, in a large wind farm some downstream turbines may undoubtedly be operating in the merged wake of several upstream turbines. It would therefore be recommended to evaluate the applicability of existing wake models in closelyspaced configurations and to modify their implementation if necessary to reflect the wake merging characteristics that occur in such configurations.

Blockage effects were evaluated in this work in the context of a rotor in a closed test section and investigated the impact of blockage on the near wake region. The effect of blockage on the speed and turbulence intensity profiles in the far wake should be evaluated in a future measurement campaign. This experiment could be conducted in a wind tunnel using HWA or PIV or in a water channel with the existing rotors using an acoustic doppler velocimeter to record the instantaneous flow speed field in the turbine wake. As it is felt that the currently-used definition of blockage for wind turbines may not adequately characterise blockage effects, a more comprehensive definition could be investigated that accounts for the tunnel aspect ratio. the rotor thrust coefficient. and the frontal area of the rotor. 
The current experiments were conducted with small-scale models that had a rotational speed that was allowed to vary with the incident wind speed. It would be very valuable to evaluate some of the closely-spaced configurations using larger-scale wind turbines. This may allow the concept to be evaluated using fixed-speed and variable-speed wind turbines in order to establish the most beneficial application of the research. This work could be complemented using actuator line LES computations of certain configurations.

One aspect of the research that could not be investigated with the available singlenormal hot-wire probe was the nature of the three-dimensional flow upstream of the rotor planes and near the blade tips in the gap between narrowly-spaced rotors. Additional experiments could be conducted with a multi-wire hot-wire anemometer or PIV and this could be assisted using computational techniques to characterise the flow angularity and the effect of swirl in the wake in the power augmentation process. 


\section{References}

[1] "World wind energy association." http://www.wwindea.org/home/index.php. Accessed Sept 232009.

[2] "Wind farms." http://www. canwea.ca/farms/index_e.php, Accessed Dec 132012.

[3] G. Smitherman, "Green energy and green economy act." Legislative Assembly of Ontario, 2009. S.O. 2009 C.12.

[4] "Ontario Power Authority - Electricity Contracts." http://http://www.powerauthority.on.ca/current-electricity-contracts/wind-power, Accessed Dec 132012.

[5] Ontario Power Authority. "Feed-in tarrif program: Program overview." tech. rep.. Ontario Power Authority. 2010.

[6] F. Amin, "Ontario's feed-in tarrif program two-year review report," tech. rep., Government of Ontario, 2012.

[7] "Independent Electricity System Operator." http://i eso.ca. Accessed Dec 132012.

[8] J. G. Leishman. "Challenges in modeling the unsteady aerodynamics of wind turbines," Wind Energy. vol. 5, pp. 85-132. 2002. 
[9] E. Hau. Wind Turbines: Fundamentals, Technologies, Application and Economics. Springer. 2nd ed.. 2005.

[10] R. Barthelmie. S. Frandsen, K. Hansen. J. Schepers. K. Rados. W. Schlez. A. Neubert. L. Jensen, and S. Neckelmann. "Modelling the impact of wakes on power output at Nysted and Horns Rev.' in Proc. of the European Wind Energy Conference. 2009.

[11] Dillon Consulting Limited, "Talbot wind farm environmental review report/environmental impact statement - Appendix A: Turbine coordinates.." tech. rep., Renewable Energy Systems Canada Inc.. 2009.

[12] "National Climate Data and Information Archive, Environment Canada." http://www.climate.weatheroffice.gc.ca/climateData/canada_e.html, Accessed Dec 102012.

[13] "Average weather in January for Chatham-Kent, Ontario, Canada." http://weatherspark. com/averages/27618/1/Chatham-Kent-Ontario-Canada, Accessed Dec 132012.

[14] Environment Canada, "Canadian wind energy atlas." http://http://www.windatlas.ca/en/nav.php. Accessed Jan. 11 2013. Last updated July 29, 2008.

[15] D. Medici, "Wind turbine wakes - control and vortex shedding," tech. rep.. KTH Mechanics Royal Institute of Technology. 2004.

[16] L. Chamorro and F. Porté-Agel. "A wind-tunnel investigation of wind-turbine wakes: Boundary-layer turbulence effects." Boundary-Layer Meteorology. vol. 132. pp. 129149,2009 .

[17] H. Snel, G. Schepers, and B. Montgomerie, "The MEXICO project (Model Experiments in Controlled Conditions): The database and first results of data processing 
and interpretation." in The Science of Making Torque from Wind. Journal of Physics: Conference Series 75. 2007.

[18] J. Ross and J. Ainslie. "Wake measurements in clusters of model wind turbines using laser doppler anemometry.", in Proc. of the Third BWEA Wind Energy Conference. 1981.

[19] J. Vermeer. L., J. Sørensen. and A. Crespo, "Wind turbine wake aerodynamics." Progress in Aerospace Sciences, vol. 39, pp. 467-510. 2003.

[20] S. McTavish. "Advanced aeroelastic simulations for practical fixed-wing and rotarywing applications," Master's thesis, Carleton University, 2008.

[21] B. Sanderse, "Aerodynamics of wind turbine wakes." Tech. Rep. ECN-e-09-016. Energy Research Centre of the Netherlands (ECN), 2009.

[22] J. F. Ainslie, "Calculating the flowfield in the wake of wind turbines," Journal of Wind Engineering and Industrial Aerodynamics, vol. 27. pp. 213-224, 1988.

[23] M. Adaramola and P- $\AA$. Krogstad. "Experimental investigation of wake effects on wind turbine performance." Renewable Energy. vol. 36. pp. 2078-2086, 2011.

[24] J. Ainslie, U. Hassan, H. Parkinson, and G. Taylor. "A wind tunnel investigation of the wake structure within small wind turbine farms," in Proc. of the European Wind Energy Conference, pp. 547-551, 1989.

[25] B. Akay, S. Ferreira, G. van Bussel, and G. Tescione. "Experimental and numerical investigation of the effect of rotor blockage on wake expansion," in Proc. of Torque 2010, The Science of Making Torque from Wind, 2010.

[26] A. Al-Abadi. Ö. Ertunç. P. Epple. W. Koerbel. and A. Delgado. "Development of an experimental setup for double rotor HAWT investigation." in Proc. of ASME Turbo Expo 2012. no. GT2012-70032. 2012. 
[27] P. Alfredsson and J. Dahlberg. "A preliminary wind tunnel study of windmill wake dispersion in various flow conditions." Tech. Rep. Technical Note AU-1499. FFA The Aeronautical Research Institute of Sweden. 1979. Part 7.

[28] S. Aubrun, P. Devinant, and G. Espana, "Physical modelling of the far wake from wind turbines. Application to wind turbine interactions." in Proc. of the European. Wind Energy Conference, 2007.

[29] S. Aubrun, S. Loyer, G. Espana, P. Hayden. and P. Hancock. "Experimental study on the wind turbine wake meandering with the help of a non-rotating simplified model and of a rotating model." in Proc. of the 49th AIAA Aerospace Sciences Meeting includeing the New Horizons Forum and Aerospace Exposition, Paper AIAA 2011$460,2011$.

[30] A. Bahaj, A. Molland. J. Chaplin, and W. Batten. "Power and thrust measurements of marine current turbines under various hydrodynamic flow conditions in a cavitation tunnel and a towing tank," Renewable Energy, vol. 32, pp. 407-426. 2007.

[31] S. Barber. N. Chokani, and R. Abhari. "Effect of wake flow non-uniformity on wind turbine performance and aerodynamics," in Proc. of ASME Turbo Expo 2011. no. GT2001-46230, 2011.

[32] T. Burdett and K. Van Treuren, "Scaling small-scale wind turbines for wind tunnel testing," in Proc. of ASME Turbo Expo 2012. no. GT2012-68359. 2012.

[33] T. Burdett and K. Van Treuren, "A theoretical and experimental comparison of optimizing angle of twist using BET and BEMT," in Proc. of ASME Turbo Expo 2012, no. GT2012-68350, 2012. 
[34] L. Chamorro and F. Porté-Agel. "Effects of thermal stability and incoming boundarylayer flow characteristics on wind-turbine wakes: A wind-tunnel study." BoundaryLayer Meteorology. vol. 136. pp. 515-533. 2010.

[35] T. Chen and L. Liou, "Blockage corrections in wind tunnel tests of small horizontalaxis wind turbines." Experimental Thermal and Fluid Science. vol. 35. pp. 565-569. 2011.

[36] T. Cho and C. Kim, "Wind tunnel test results for a $2 / 4.5$ scale MEXICO rotor," Renewable Energy, vol. 42, pp. 152-156, 2012.

[37] H. Dan-mei and D. Zhao-hui, "Near wake of a model horizontal-axis wind turbine." Journal of Hydrodynamics, vol. 21, pp. 285-291, 2009.

[38] I. Grant, P. Parkin, and X. Wang, "Optical vortex tracking studies of a horizontal axis wind turbine in yaw using laser-sheet, flow visualisation," Experiments in Fluids, vol. 23, pp. 513-519, 1997.

[39] I. Grant, M. Mo, X. Pan. P. Parkin. J. Powell, H. Reinecke, K. Shuang, F. Coton. and D. Lee, "An experimental and numerical study of the vortex filaments in the wake of an operational, horizontal-axis, wind turbine," Journal of Wind Engineering and Industrial Aerodynamics, vol. 85, pp. 177-189, 2000.

[40] W. Haans. T. Sant, G. van Kuik, and G. van Bussel. "Measurement of tip vortex paths in the wake of a HAWT under yawed flow conditions," Journal of Solar Energy Engineering- T ASME, vol. 127, pp. 456-463, 2005.

[41] M. Hossain. H. Hirahara. Y. Nonomura. and M. Kawahashi. "The wake structure in a 2D grid installation of the horizontal axis micro wind turbines." Renewable Energy. vol. 32, pp. 2247-2267, 2007. 
[42] T. Maeda. Y. Kamada. J. Murata. T. Mizokoshi. S. Yonekura, and T. Kogaki. "Wind tunnel study on load fluctuation of horizontal axis wind turbine in wake," in Proc. of the European Wind Energy Conference. 2010.

[43] T. Maeda, Y. Kamada, J. Murata, S. Yonekura. T. Ito. A. Okawa. and T. Kogaki. "Wind tunnel study on wind and turbulence intensity profiles in wind turbine wake." Journal of Thermal Science, vol. 20. no. 2. pp. 127-132. 2011.

[44] F. Massouh and I. Dobrev, "Exploration and numerical simulation of wind turbine wake," International Scientific Journal for Alternative Energy and Ecology. vol. 62. no. 6 , pp. 163-168. 2007.

[45] D. Medici and H. Alfredsson, "Wind turbine near wakes and comparisons to the wake behind a disc," in Proc. of the 43rd AIAA Aerospace Sciences Meeting and Exhibit. no. AIAA 2005-595, 2005.

[46] L. Myers and A. Bahaj, "An experimental investigation simulating flow effects in first generation marine current energy conerter arrays." Renewable Energy. vol. 37. pp. $28-36,2012$.

[47] D. Neff, R. Meroney, E. McCarthy, and E. Davis, "Upstream and lateral wind turbine wake effects on nearby wind turbine performance." Journal of Wind Engineering and Industrial Aerodynamics, vol. 36, pp. 1405-1414, 1990.

[48] D. Simms, S. Schreck, M. Hand, and L. Fingersh, "NREL Unsteady Aerodynamics Experiment in the NASA-Ames wind tunnel: A comparison of predictions to measurements," NREL/TP-500-29494, National Renewable Energy Laboratory. Golden. Colorado, USA. 2001. 
[49] J. Whale. C. Anderson. R. Bareiss. and S. Wagner. "An experimental and numerical study of the vortex structure in the wake of a wind turbine," Journal of Wind Engineering and Industrial Aerodynamics. vol. 84. pp. 1-21. 2000.

[50] J. Xiao, J. Wu. L. Chen, and Z. Shi. "Particle image velocimetry (PIV) measurements of tip vortex wake structure of wind turbine," Applied Mathematics and Mechanics (English Edition), vol. 32, no. 6, pp. 729-738, 2011.

[51] Z. Yang, P. Sarkar, and H. Hu, "An experimental investigation on the wake characteristics of a wind turbine in an atmospheric boundary layer wind," in Proc. of the 29th AIAA Applied Aerodynamics Conference. no. AIAA 2011-3815, 2011.

[52] L. Vermeer, "A review of wind turbine wake research at TUDelft." in Proc. of the 39th Aerospace Sciences Meeting and Exhibit, 20th ASME Wind Energy Symposium. 2001.

[53] J. Schepers, K. Boorsma, T. Cho, S. Gomez-Iradi, P. Schaffarczyk, A. Jeromin. W. Shen, K. Lutz, K. Meister, B. Stoevesandt, S. Schreck, D. Micallef, R. Pereira. T. Sant, H. Madsen, and N. Sørensen, "Final report of IEA Task 29, Mexnext (phase 1): Analysis of Mexico wind tunnel measurements.." Tech. Rep. ECN-E-12-004. Energy Research Center of the Netherlands. February 2012.

[54] S. Barber, Y. Wang, S. Jafari, N. Chokani, and R. Abhari, "The impact of ice formation on wind turbine performance and aerodynamics," in Proc. of the European Wind Energy Conference, pp. 28-32. 2010.

[55] M. Hand, D. Simms, L. Fingersh. D. Jager, J. Cotrell, S. Schreck, and S. Larwood. "Unsteady Aerodynamics Experiment Phase VI: Wind tunnel test configuration and available data campaigns," NREL/TP-500-29955, National Renewable Energy Laboratory, Golden, Colorado, USA, 2001. 
[56] S. Schreck, "The NREL full-scale wind tunnel experiment - Introduction to the special issue," Wind Energy. vol. 5. 2002.

[57] P- $\AA$. Krogstad and P. Eriksen. "Blind test" calculations of the performance and wake development for a model wind turbine." Renewable Energy. vol. 50, pp. 325-333. 2013.

[58] P- $\AA$. Krogstad and M. Adaramola, "Performance and near wake measurements of a model horizontal axis wind turbine." Wind Energy. vol. 15. pp. 743-758. 2012.

[59] L. Myers and A. Bahaj. "Experimental analysis of the flow field around horizontal axis tidal turbines by use of scale mesh disk rotor simulators," Ocean Engineering. vol. 37, pp. 218-227, 2010.

[60] M. Werle, "Wind turbine wall-blockage performance corrections." Journal of Propulsion and Power, vol. 26, pp. 1317-1321. Technical Note 2010.

[61] W. Rae and A. Pope. Low-speed wind tunnel testing. John Wiley \& Sons, 2nd ed., 1984.

[62] J. Bartl. "Wake measurements behind an array of two model wind turbines." Master's thesis, KTH Industrial Engineering and Management, 2011.

[63] I. Ross and A. Altman, "Wind tunnel blockage corrections: Review and application to Savonius vertical-axis wind turbines," Journal of Wind Engineering and Industrial Aerodynamics, vol. 99. pp. 523-538, 2011.

[64] L. Chamorro, R. Arndt, and F. Sotiropoulos, "Reynolds number dependence of turbulence statistics in the wake of wind turbines." Wind Energy. vol. 15, pp. 733-742, 2012.

[65] N. Troldborg, J. Sørensen. and R. Mikkelsen. "Actuator line simulation of wake of wind turbine operating in turbulent inflow," in Proc. of The Science of Making Torque from Wind, vol. 75, pp. 1-15, Journal of Physics: Conference Series, 2007. 
[66] J. Sørensen. W. Shen. and X. Munduate. "Analysis of wake states by a full-field actuator disc model," Wind Energy, vol. 1. pp. 73-88. 1998.

[67] C. Tsalicoglou. S. Barber. N. Chokani. and R. Abhari. "Effect of flow inclination on wind turbine performance," in Proc. of ASME Turbo Expo 2012, no. GT2012-68473. 2012.

[68] J. Meyers and C. Meneveau, "Optimal turbine spacing in fully developed wind farm boundary layers," Wind Energy, vol. 15. pp. 305-317, 2012.

[69] Hatch TM " "South Kent Wind Project, Proposed Draft Site Plan," Sept 2011.

[70] T. Nishino and R. Willden. "The efficiency of an array of tidal turbines partially blocking a wide channel," Journal of Fluid Mechanics. vol. 708. pp. 596-606. 2012.

[71] T. Nishino and R. Willden, "Effects of 3-D channel blockage and turbulent wake mixing on the limit of power extraction by tidal turbines," International Journal of Heat and Fluid Flow, vol. 37, pp. 123-135, 2012.

[72] S. Turnock, A. Phillips. J. Banks, and R. Nicholls-Lee. "Modelling tidal current turbine wakes using a coupled RANS-BEMT approach as a tool for analysing power capture of arrays of turbines," Ocean Engineering, vol. 38, pp. 1300-1307, 2011.

[73] M. Shives. "Hydrodynamic modeling. optimization and performance assessment for ducted and non-ducted tidal turbines." Master's thesis. University of Victoria. 2011.

[74] S. McTavish, D. Feszty, and F. Nitzsche. "The development of a scaled wind farm laboratory for undergraduate education," in Proc. of the 12th Americas Conference on Wind Engineering, no. 123, 2013.

[75] L. Chamorro and F. Porté-Agel. "Turbulent flow inside and above a wind farm: a wind-tunnel study," Energies, vol. 4, pp. 1916-1936. 2011. 
[76] L. Chamorro, R. Arndt, and F. Sotiropoulos. "Turbulent flow properties around a staggered wind farm," Boundary-Layer Meteorology. vol. 141. pp. 349-367. 2011.

[77] A. Ozbay, "Experimental investigations on the wake interferences of multiple wind turbines," Master's thesis, Iowa State University. 2012.

[78] G. Corten and P. Schaak. "More power and less loads in wind farm: 'Heat and Flux'," in Proc. of the European Wind Energy Conference, 2004.

[79] G. Corten, P. Schaak, and T. Hegberg, "Turbine interaction in large offshore wind farms: wind tunnel measurements," Tech. Rep. ECN-C-04-048, Energy Center of the Netherlands, 2004.

[80] R. Barthelmie, S. Frandsen, O. Rathmann. K. Hansen, E. Politis, J. Prospathopoulos. J. Schepers, K. Rados, D. Cabezón, W. Schlez, A. Neubert, and M. Heath. "Flow and wakes in large wind farms: Final report for UpWind WP8," tech. rep.. Risø DTU National Laboratory for Sustainable Energy, 2011.

[81] H. Snel, "Review of the present status of rotor aerodynamics." Wind Energy. vol. 1. pp. $46-69,1998$.

[82] H. Snel, "Review of aerodynamics for wind turbines," Wind Energy, vol. 6, pp. 203$211,2003$.

[83] M. Hansen, J. Sørensen, S. N. Voutsinas, S., and H. Madsen, "State of the art in wind turbine aerodynamics and aeroelasticity." Progress in Aerospace Sciences. vol. 42. 2006.

[84] D. Molenaar and S. Dijkstra, "State-of-the-art of wind turbine design codes: Main features overview for cost-effective generation." Wind Engineering. vol. 23. 1999.

[85] H. Glauert, "Airplane propellers," in Aerodynamic Theory (W. F. Durand. ed.). vol. 4. (Berlin, Germany). pp. 169-360, Springer-Verlag, 1935. 
[86] M. Hansen. Aerodynamics of Wind Turbines. New York: Earthscan Publications Ltd.. 1st ed., 2001.

[87] T. Burton, D. Sharpe, N. Jenkins, and E. Bosisanyi. Wind Energy Handbook. New York: John Wiley and Sons, 2001.

[88] J. G. Leishman, Principles of Helicopter Aerodynamics. Cambridge Aerospace Series. New York: Cambridge University Press. 2nd ed., 2006.

[89] S. Gupta and J. Leishman, "Comparison of momentum and vortex methods for the aerodynamic analysis of wind turbines," in Proc. of the $4.3 \mathrm{rd}$ AIAA Aerospace Sciences Meeting and Exhibit, Paper AIAA 2005-594. 2005.

[90] D. Marten, J. Wendler, G. Pechlivanoglou, C. Nayeri, and C. Paschereit. "Development and application of a simulation tool for vertical and horizontal axis wind turbines," in Proc. of ASME Turbo Expo, no. GT2013-94979, 2013.

[91] G. Schepers, R. Barthelmie, K. Rados, B. Lange. and W. Schlez, "Large off-shore windfarms: Linking wake models with atmospheric boundary layer models," Wind Engineering, vol. 25, pp. 307-316, 2001.

[92] A. Crespo, J. Hernandez, and S. Frandsen. "Survey of modelling methods for wind turbine wakes and wind farms." Wind Energy, vol. 2. pp. 1-24. 1999.

[93] P. Lissaman, "Energy effectiveness of arbitrary arrays of wind turbines." AIAA Journal, 1979.

[94] I. Katic, J. Hostrup, and N. Jensen, "A simple model for cluster efficiency." in Proc. of the European Wind Energy Conference, pp. 407-410. 1986.

[95] S. Voutsinas, K. Rados, and A. Zervos. "On the analysis of wake effects in wind parks," Wind Engineering. vol. 14, pp. 204-219. 1990. 
[96] "GH WindFarmer validation report." tech. rep.. Garrad Hassan. Bristol. England. 2003.

[97] W. Schlez, A. Umana. R. Barthelmie. G. Larsen. K. Rados. B. Lange. G. Schepers. and T. Hegberg. "ENDOW: Improvement of wake models within offshore wind farms." Wind Engineering. vol. 25, no. 5, pp. 281-287, 2001.

[98] R. Barthelmie, J. Schepers, S. van der Pijl, O. Rathmann, S. Frandsen, D. Cabezón, E. Politis, J. Prospathopoulos, K. Rados, K. Hansen, W. Schlez. J. Phillips. and A. Neubert, "Flow and wakes in complex terrain and offshore: Model development and verification in UpWind," in Proc. of the European Wind Energy Conference. 2007.

[99] D. Renkema, "Validation of wind turbine wake models: using wind farm data and wind tunnel measurements," Master's thesis, Delft University of Technology. 2007.

[100] J. Robison, D., F. Coton. R. Galbraith, and M. Vezza, "Application of a prescribed wake aerodynamic prediction scheme to horizontal axis wind turbines in axial flow." Wind Engineering, vol. 19, no. 1, pp. 41-51, 1995.

[101] S. Lain, J. Garcia, and R. Aliod, "Development of a lifting surface - 2D panel method to compute the three-dimensional pressure distribution over the blade of a horizontaaxis wind turbine," Wind Engineering, vol. 19, 1995.

[102] T. Wang and F. Coton, "An unsteady aerodynamic model for HAWT performance including tower shadow effects." Wind Engineering, vol. 23, 1999.

[103] S. Schmitz and J.-J. Chattot. "Method for aerodynamic analysis of wind turbines at peak power," AIAA Journal of Propulsion and Power - Technical Notes, vol. 23. no. 1, 2007. 
[104] S. Breton, F. Coton, and G. Moe, "A study on rotational effects and different stall delay models using a prescribed wake vortex scheme and NREL Phase VI experiment data." Wind Energy, vol. 11, pp. 459-482, 2008.

[105] E. Duque, M. Burklund. and W. Johnson. "Navier-Stokes and comprehensive analysis performance predictions of the NREL Phase VI experiment." in Proc. of the 41 st AIAA Aerospace Sciences Meeting and Exhibit, Paper AIAA 2003-355, 2003.

[106] S. Gupta. Development of a time-accurate viscous Lagrangian vortex wake model for wind turbine applications. PhD thesis, University of Maryland. 2006.

[107] S. Gupta and J. Leishman, "Performance predictions of the NREL phase VI combined experiment rotor using a free-vortex wake model," in Proc. of the 44 th AIAA Aerospace Sciences Meeting and Exhibit, Paper AIAA 2006-390, 2006.

[108] S. Gupta and J. Leishman, "Validation of a free-vortex wake model for wind turbines in yawed flow," in Proc. of the 44th AIAA Aerospace Sciences Meeting and Exhibit. Paper AIAA 2006-389, 2006.

[109] S. Gupta and J. Leishman. "Dynamic stall modeling of the S809 airfoil and comparison with experiments," in Proc. of the 44th AIAA Aerospace Sciences Meeting and Exhibit. Paper AIAA 2006-196, 2006.

[110] S. Voutsinas, "Vortex methods in aeronautics: how to make things work," International Journal of Computational Fluid Dynamics, vol. 20. pp. 3-18, 2006.

[111] A. Zervos, S. Huberson, and A. Hemon. "Three-dimensional free wake calculation of wind turbine wakes," Journal of Wind Engineering and Industrial Aerodynamics, vol. 27, pp. 65-76, 1988. 
[112] S. Voutsinas, M. Belessis. and S. Huberson. "Dynamic inflow effects and vortex particle methods." in Proc. of the European Community Wind Energy Conference. P. 428-431, 1993.

[113] D. Willis, J. Peraire, and J. White, "A combined pFFT-multipole tree code, unsteady panel method with vortex particle wakes," International Journal for Numerical Methods in Fluids, vol. 53, pp. 1399-1422, 2007.

[114] D. Opoku. "Aeroelastic and aeroacoustic modelling of rotorcraft," Master's thesis. Carleton University, 2002.

[115] D. Opoku, D. Triantos, F. Nitzsche, and S. Voutsinas, "Rotorcraft aerodynamic and aeroacoustic modelling using vortex particle methods." in Proc. of the ICAS 2002 Congress, The International Council of the Aeronautical Sciences, 2002.

[116] D. Opoku and F. Nitzsche, "Acoustic validation of a new code using particle wake aerodynamics and geometrically-exact beam structural dynamics." in Proc. of the 29th European Rotorcraft Forum. 2003.

[117] C. Cesnik, D. Opoku, F. Nitzsche, and T. Cheng, "Active twist rotor blade modelling using particle-wake aerodynamics and geometrically exact beam structural dynamics." Journal of Fluids and Structures, vol. 19. pp. 651-668. 2004.

[118] S. McTavish, D. Feszty, and F. Nitzsche, "Aeroelastic simulations of the NREL Phase VI wind turbine using a discrete vortex method coupled with a nonlinear beam model," in Proc. of the European Wind Energy Conference, 2009.

[119] S. McTavish, D. Feszty, and F. Nitzsche. "Aeroelastic simulations of the NREL Phase VI wind turbine using a discrete vortex method." in Proc. of the Canadian Aeronautics and Space Institute AERO Conference. 2009. 
[120] T. Fletcher. R. Brown. D. Kim. and O. Kwon. "Predicting wind turbine blade loads using vorticity transport and RANS methodologies.". in Proc. of the European Wind Energy Conference, 2009.

[121] S. McTavish. D. Feszty, and T. Sankar. "Steady and rotating computational fluid dynamics simulations of a novel vertical axis wind turbine for small-scale power generation." Renewable Energy, vol. 41. pp. 171-179. 2012.

[122] P. Jaohindy, S. McTavish, F. Garde, and A. Bastide, "An analysis of the transient forces acting on savonius rotors with different aspect ratios," Renewable Energy, vol. 55, pp. 286-295, 2013.

[123] N. Sørensen and M. Hansen, "Rotor performance prediction using a Navier-Stokes method," in American Institue of Aeronautics and Astronautics, Paper AIAA 1998$0025,1998$.

[124] E. Duque. C. van Dam, and C. Shannon. "Navier-Stokes simulations of the NREL Combined Experiment Phase II rotor." AIAA Journal. 1999.

[125] N. Sørensen, J. Michelsen, and S. Schreck, "Navier-Stokes predictions of the NREL Phase VI rotor in the NASA Ames $80 \mathrm{ft} \times 120 \mathrm{ft}$ wind tunnel," Wind Energy, vol. 5. 2002 .

[126] F. Massouh, T. Dobrev, and M. Rapin, "Experimental and numerical survey in the wake of a wind turbine," in Proc. of the 45 th AIAA Aerospace Sciences Meeting and Exhibit, Paper AIAA 2007-423, 2007.

[127] A. Bechmann and N. Sørensen, "CFD simulation of the MEXICO rotor wake." in Proc. of the European Wind Energy Conference. 2009. 
[128] P-E. Réthoré. N. Sørensen. F. Zahle. A. Bechmann. and H. Madsen. "MEXICO wind tunnel and wind turbine modelled in CFD." in Proc. of the 29th AIAA Applied Aerodynamics Conference, no. AIAA 2011-3373, 2011.

[129] S. Schmitz and J. Chattot, "Characterization of 3D effects for the parked and rotating NREL Phase VI wind turbine." in Proc. of the 44th AIAA Aerospace Sciences Meeting and Exhibit, Paper AIAA 2006-392, 2006.

[130] J. Mo, A. Choudhry. M. Arjomandi, and Y. Lee. "Large eddy simulation of the wind turbine wake characteristics in the numerical wind tunnel model." Journal of Wind Engineering and Industrial Aerodynamics. vol. 112, pp. 11-24, 2013.

[131] C. Masson, I. Ammara, and I. Paraschivoiu, "An aerodynamic method for the analysis of isolated horizontal-axis wind turbines," International Journal of Rotating Machines, vol. 3, pp. 21-32, 1997.

[132] M. Torresi, B. Fortunato, and S. Camporeale, "An efficient 3D CFD model for the analysis of the flow field around Darrieus rotors." in Proc. of ASME Turbo Expo. no. GT2013-95564, 2013.

[133] C. Masson, I. Ammara, C. Leclerc, and I. Paraschivoiu, "Numerical simulations of the three-dimensional turbulent flow in a wind park." AIAA Journal - AIAA 98-0058. 1998.

[134] I. Ammara, C. Leclerc, and C. Masson, "A viscous three-dimensional differential/actuator-disk method for aerodynamic analysis of wind farms." Journal of Solar Energy - T ASME, vol. 124, no. 4, pp. 345-356. 2002.

[135] N. Sørensen, J. and C. Kock, "A model for unsteady rotor aerodynamics," Journal of Wind Engineering and Industrial Aerodynamics. vol. 39. pp. 139-149. 1992. 
[136] S. Breton. S. Watters. and C. Masson. "Aerodynamic simulations of a three-bladed wind turbine rotor using the actuator surface method." in Proc. of the European Wind Energy Conference. 2009.

[137] R. Mikkelsen. Actuator Disc Methods Applied to Wind Turbines. PhD thesis. Technical University of Denmark. 2003.

[138] N. Troldborg, J. Sørensen, and R. Mikkelsen, "Numerical simulations of wakes of wind turbines in wind farms," in Proc. of the European Wind Energy Conference, 2006.

[139] R. Mikkelsen, J. Sørensen, S. Øye, and N. Troldborg, "Analysis of power enhancement for a row of wind turbines using the actuator line technique," in Proc. of The Science of Making Torque from the Wind, vol. 75, Journal of Physics: Conference Series. 2007.

[140] N. Troldborg, J. Sørensen, and R. Mikkelsen, "Numerical simulations of wakes of wind turbines operating in sheared and turbulent inflow," in Proc. of the European Wind Energy Conference, 2009.

[141] M. Churchfield, Y. Li, and P. Moriary, "A Large-Eddy Simulation study of wake propagaton and power production in an array of tidal-current turbines," Tech. Rep. NREL/CP-5000-51765, National Renewable Energy Laboratory, 2011.

[142] G. Xu, Computational Studies of Horizontal Wind Turibnes. PhD thesis, Georgia Institute of Technology, 2001.

[143] S. Benjanirat and L. Sankar, "Recent improvements to a combined Navier-Stokes full potential methodology for modeling horizontal axis wind turbines," in Proc. of the 42nd AIAA Aerospace Sciences Meeting and Exhibit, Paper AIAA 2004-830. 2004.

[144] S. Schmitz and J. Chattot, "Wind turbine blade aerodynamics of the NREL Phase VI rotor near peak power," in Proc. of the 2.3rd AIAA Applied Aerodynamics Conference. Paper AIAA 2005-4850, 2005. 
[145] S. Schmitz and J. Chattot. "A parallelized coupled Navier-Stokes/vortex-panel solver," Journal of Solar Energy Engineering. vol. 127. pp. 475-487. 2005.

[146] P. Anusonti-Inthra and M. Floros, "Coupled CFD and particle vortex transport method: Wing performance and wake validations." in Proc. of the 38th Fluid Dynamics Conference and Exhibit. Paper AIAA 2008-4177. 2008.

[147] G. Oxley, A 2-D Hybrid Euler-Compressible Vortex Particle Method For Transonic Rotorcraft Flows. PhD thesis, Carleton University. 2009.

[148] E. Laitone, "Wind tunnel tests of wings at Reynolds numbers below 70000 ," Experiments in Fluids, vol. 23, pp. 405-409, 1997.

[149] M. Selig, J. Guglielmo, A. Broeren, and P. Giguère, Summary of low-speed airfoil data, vol. 1. Virginia Beach, VA: SoarTech Publications, 1995.

[150] G. Spedding and J. McArthur, "Span efficiencies of wings at low Reynolds numbers." Journal of Aircraft, vol. 47, pp. 120-128, January-February 2010.

[151] J. McArthur, Aerodynamics of wings at low Reynolds numbers: boundary layer separation and reattachment. PhD thesis, University of Southern California, 2008.

[152] Rolling Hills Research Corporation, "Model 2436 flow visualization research water tunnel operations and maintenance manual," tech. rep., 2007.

[153] W. Merzkirch, Flow Visualization. Academic Press. Inc.. 1987.

[154] L. Aurahs. C. Kasper, M. Kürner, M. Rose. S. Staudacher. and J. Gier. "Water flow model turbine flow visualization study of the unsteady interaction of secondary flow vortices with the downstream rotor," in Proc. of the Institution of Mechanical Engineers, Part A: Journal of Power and Energy. vol. 223, pp. 677-686. 2009.

[155] C. Arends, "A wind tunnel study of atmospheric boundary layer effects on roof mounted solar panel arrays," Master's thesis. Carleton University, 2011. 
[156] S. Tavoularis. Measurement in fluid mechanics. Cambridge University Press. 2005.

[157] J. Whelan. M. Graham. and J. Peiró, "A free-surface and blockage correction for tidal turbines," Journal of Fluid Mechanics, vol. 624, pp. 281-291, 2009.

[158] R. Kind, "Wind tunnel research notes.".

[159] J. Wyngaard, Turbulence in the Atmosphere. Cambridge University Press, 2010.

[160] H. Irwin, "The design of spires for wind simulation." Journal of Wind Engineering and Industrial Aerodynamics, vol. 7. pp. 361-366, 1981. Short Communication.

[161] A.A. Lab Systems Ltd, AN-1005 Hot wire E' film anemometry system: User's Manual.

[162] H. Bruun, Hot-Wire Anemometry: Principles and Signal Analysis. Oxford University Press, 1995.

[163] G. Oxley, F. Nitzsche, and D. Feszty, "Smart spring control of vibration on helicopter rotor blades," Journal of Aircraft, vol. 46. no. 2, pp. 692-696, 2009.

[164] S. Ivanell, R. Mikkelsen, J. Sørensen, and D. Henningson. "Stability analysis of the tip vortices of a wind turbine," Wind Energy, vol. 13. no. 8, pp. 705-715. 2010.

[165] W. Haans, T. Sant. G. van Kuik. and G. van Bussel, "Measurement and modelling of tip vortex paths in the wake of a HAWT under yawed flow conditions." in Proc. of the 43rd AIAA Aerospace Sciences Meeting and Exhibit. Paper AIAA 2005-590. 2005.

[166] N. Hung and H. Ngoc, "Experimental study of laminar separation phenomenon combining with numerical calculations," Vietnam Journal of Mechanics, VAST. vol. 33, pp. $95-104,2011$.

[167] O. Lehmkuhl, A. Baez, I. Rodríguez, and C. Pérez-Segarra, "Direct numerical simulation and large-eddy simulations of the turbulent flow around a NACA-0012 
airfoil." in Proc. of 7th International Conference on Computational Heat and Mass Transfer, International Centre for Applied Thermodynamics. July 2011.

[168] H. Tanaka, "Flow visualization and PIV measurements of laminar separation bubble oscillating at low frequency on an airfoil near stall." in Proc. of 24 th International Congress of the Aeronautical Sciences. August 2004.

[169] R. Sheldahl and P. Klimas, "Aerodynamic characteristics of seven airfoil sections through 180 degrees angle of attack for use in aerodynamic analysis of vertical axis wind turbines," Tech. Rep. SAND80-2114, Sandia National Laboratories, 1981.

[170] R. Barthelmie, L. Folkerts, G. Larsen, K. Rados, S. Pryor, S. Frandsen, B. Lange, and G. Schepers, "Comparison of wake model simulations with offshore wind turbine wake profiles measured by sodar," Journal of Atmospheric and Oceanic Technology, vol. 23, pp. $888-901,2005$.

[171] D. Medici and P. Alfredsson, "Measurements behind model wind turbines: Further evidence of wake meandering," Wind Energy. vol. 11, pp. 211-217. 2008. Short Communication.

[172] S. McTavish, D. Feszty, and F. Nitzsche, "An experimental assessment of blockage and Reynolds number effects on wind turbine wake development." in Proc. of ASME TurboExpo, no. GT2012-69335, 2012.

[173] S. McTavish, D. Feszty, and F. Nitzsche, "An experimental and computational assessment of blockage effects on wind turbine wake development." Wind Energy Under Review, 2012

[174] S. McTavish, D. Feszty, and F. Nitzsche. "Evaluating Reynolds number effects in small-scale wind turbine experiments." Journal of Wind Engineering and Industrial Aerodynamics - Under Review, 2012. 
[175] S. McTavish. D. Feszty, and F. Nitzsche. "A study of the performance benefits of closely-spaced lateral wind farm configurations." Renewable Energy, vol. 59, pp. 128$135,2013$.

[176] D. Feszty and S. McTavish. "Wind farm, and corresponding methods.." United States Patent and Trademark Office. Provisional Patent Application 61/659.673.

[177] S. McTavish, D. Feszty, and F. Nitzsche, "Evaluating field blockage effects in closelyspaced lateral wind farm configurations." in Proc. of the 12th Americas Conference on Wind Engineering, no. 53, 2013. 